\title{
Microstructural control of ZnO films deposited by RF magnetron sputtering for ultrasound transducers
}

\author{
by \\ Dayna-Maree Kivell
}

\author{
A thesis \\ submitted to the Victoria University of Wellington \\ in fulfilment of the \\ requirements for the degree of \\ Master \\ in Engineering. \\ Victoria University of Wellington \\ 2013
}





\begin{abstract}
The aim of this study was to develop a deposition process using RF magnetron sputtering for the production of zinc oxide $(\mathrm{ZnO})$ thin films on glass substrates. These $\mathrm{ZnO}$ films were to be used as the active piezoelectric element in very high frequency ultrasound transducers (>300 $\mathrm{MHz}$ ). In order to achieve piezoelectric activity the films had to be oriented with the c-axis of the $\mathrm{ZnO}$ grains perpendicular to the substrate surface. At the same time, a moderately high, at least $1 \mu \mathrm{m} / \mathrm{hr}(17 \mathrm{~nm} / \mathrm{min})$ deposition rate was required for the production of practical devices. Prior to a full investigation into the sputtering parameters, an initial evaluation of the HHV Auto500 RF magnetron sputter coater system was performed. Using the original chamber configuration it was not possible to deposit $\mathrm{ZnO}$ at the required deposition rates. A modification of the growth chamber to allow a reduced target-substrate distance was successful in producing $\mathrm{ZnO}$ films at the required deposition rates. A systematic study into the deposition parameters and their effect on the $\mathrm{ZnO}$ film quality and deposition rates was then performed and it was found that strong c-axis oriented films could be deposited only when depositing at rates below $15 \mathrm{~nm} / \mathrm{min}$ at a low substrate temperature $\left(<50^{\circ} \mathrm{C}\right)$. Depositions above this rate resulted in the growth of polycrystalline films. A two-step deposition process was designed to preserve c-axis orientation at high deposition rates up to $28 \mathrm{~nm} / \mathrm{min}$. The $\mathrm{ZnO}$ films were found to be highly strained due to inherent stress from the sputtering process. The deposition pressure was identified as the most critical deposition parameter for stress control. It was found that deposition above a critical pressure of $1.2 \times 10^{-2}$ mbar was essential to prevent mechanical failure of the films. Post growth annealing
\end{abstract}


was investigated and determined to be a viable technique to relax stress and improve the crystalline quality of the films. Finally a four-step deposition process was proposed to facilitate the growth of c-axis oriented $\mathrm{ZnO}$ films at relatively high deposition rates whilst minimising film stress. 


\section{Acknowledgments}

"Research is what I'm doing when I don't know what I'm doing"

Wernher von Braun.

First and foremost I would like to give a massive thank you to Dr Gideon Gouws for his tremendous help, support and patience. His encouragement and guidance throughout this project has been much appreciated. I enjoyed discussing results, which always led to the excitement of new experiments. The joy of solving problems was indescribable.

Secondly I would like to give a big thank you to Dr Natalie Plank for all her help and support. Her time and effort was much appreciated. Good times in the lab, great experiments and excellent music.

I would also like to thank the clean room group for all the science fun and laughs we had together.

A big thanks to David Flynn for his help with the scanning electron microscope and the mechanical workshop guys who could fix just about anything.

To House Boston, you guys rock, nerds for life.

To Abigail Arulandu, my thesis writing buddy, we both went through this 
iv

crazy time together. We did it!

I would also like to thank my family and friends for their support and encouragement over this time. A special thanks to my awesome grandparents for all the fun times in the retirement village (or should I say young people's holiday resort).

Finally I would like to thank my mum and best friend, Amala Shanti. You are amazing and a truly wonderful being, thank you for everything. I love you a million red raspberries.

"The best way to predict the future is to create it"

Peter Drucker. 


\section{Contents}

1 Introduction 1

2 Background Theory $\quad 7$

2.1 History and properties of $\mathrm{ZnO} \ldots \ldots \ldots$

2.1 .1 Crystal Structure . . . . . . . . . . . . . . . . . . . . . . 9

2.2 Piezoelectricity . . . . . . . . . . . . . . 11

2.2.1 The linear piezoelectric equations . . . . . . . . 12

2.2.2 Electric and piezoelectric properties of $\mathrm{ZnO}$. . . . . 13

2.2.3 Applications of $\mathrm{ZnO}$ in piezoelectric devices . . . . 15

2.3 Functional thin films . . . . . . . . . . . . . . 16

2.3.1 Thin film growth models . . . . . . . . . . 16

2.3.2 Oriented film growth . . . . . . . . . . . . . . 18

2.3.3 Structure zone model $(\mathrm{SZM}) \ldots \ldots$. . . . . . 18

2.3.4 Residual stress in thin films . . . . . . . . . . . . 20

2.4 Depositing $\mathrm{ZnO}$ thin films . . . . . . . . . . . . . . . 21

2.4 .1 Sputtering theory . . . . . . . . . . . 21

2.4.1.1 DC sputtering ............ 22

2.4.1.2 RF sputtering . . . . . . . . . . . 25

2.4.1.3 RF magnetron sputtering . . . . . . . . . 25

2.4.2 Sputtering parameters for $\mathrm{ZnO} \ldots \ldots . . . . . .27$

2.4.3 Requirements of $\mathrm{ZnO}$ thin films for ultrasound transducer applications . . . . . . . . . . . . 27 
3 Experimental Techniques 29

3.1 Sputter Deposition System: The Auto500 . . . . . . . . . . . 29

3.2 Substrate Preparation . . . . . . . . . . . . . . . . . 32

3.2.1 Deposition Procedure . . . . . . . . . . . . 33

3.3 Thermal Annealing . . . . . . . . . . . . . . . . 34

3.4 Thin Film Characterisation Techniques . . . . . . . . . . . 37

3.4.1 Dektak thickness profiler . . . . . . . . . 37

3.4.2 UV-Visual Spectrophotometer: . . . . . . . . . 38

3.4.3 X-ray Diffraction (XRD) measurements . . . . . . . 41

3.4.4 Scanning Electron Microscopy (SEM) . . . . . . . . 48

4 Results $\quad 51$

4.1 Initial sputter system evaluation . . . . . . . . . . 52

4.1.1 Setting of chamber pressure . . . . . . . . . 53

4.1.2 Influence of RF power on deposition rate . . . . . . 55

4.1.3 Influence of Ar flow on deposition rate . . . . . . 56

4.1.4 Control of substrate temperature . . . . . . . . . 58

4.1.5 Influence of deposition parameters on film properties 59

4.1.5.1 Effects of RF power . . . . . . . . . . 62

4.1.5.2 Effects of Ar flow . . . . . . . . . . . 62

4.1.5.3 Effects of substrate temperature . . . . . . 64

4.1.5.4 Effects of substrate rotation . . . . . . . 65

4.1.6 Conclusions from initial system evaluation . . . . . . 71

4.2 Chamber modification \#1: Reduce target-to-substrate distance 72

4.2.1 Effect of reduced target-to-substrate distance on deposition rate . . . . . . . . . . . . 73

4.2.1.1 Rotating substrate holder . . . . . . . . 73

4.2.1.2 Stationary substrate holder . . . . . . . . 75

4.2.2 Effect of substrate temperature . . . . . . . . . 79

4.2 .3 Effects of RF power . . . . . . . . . . . . . 81

4.2.4 Introduction of a buffer layer structure . . . . . . 83 
4.2.5 Interdependence of film structure and surface morphology . . . . . . . . . . . . . 85

4.2.6 Effects of the buffer layer structure with substrate heating . . . . . . . . . . . . . . . 89

4.2.7 Conclusions form modification \#1 . . . . . . . . . . 91

4.3 Origin of film cracking and delamination . . . . . . . . . . 93

4.3.1 Poor substrate-film adhesion . . . . . . . . . . . 96

4.3.2 Stress in $\mathrm{ZnO}$ film . . . . . . . . . . . . . . . . . . . 96

4.3.3 Influence of deposition parameters on stress . . . . . 100

4.3.3.1 Effect of RF power . . . . . . . . . . . . . . . 100

4.3.3.2 Effect of deposition pressure . . . . . . . 103

4.3.4 Stress relaxation through post growth annealing . . . 105

4.3.5 Conclusions on stress within the $\mathrm{ZnO}$ films . . . . . . 114

4.4 Chamber modification \#2: New substrate holder . . . . . . . 115

4.4 .1 Film uniformity . . . . . . . . . . . . . . 115

4.4.2 Conclusions from modification \#2 . . . . . . . . . 120

4.5 Change in $\mathrm{ZnO}$ sputter target . . . . . . . . . . . . . . . 120

4.5.1 Change in optical appearance . . . . . . . . . . . . 120

4.5.2 Effect of Ar: $\mathrm{O}_{2}$ ratio . . . . . . . . . . . . . . 123

4.5.3 Other deposition parameters . . . . . . . . . . 125

4.5.4 Conclusions from change in $\mathrm{ZnO}$ target . . . . . . . . 125

5 Conclusions and Suggestions for future work 127

5.1 Performance and modification of the sputter system . . . . . 127

5.2 Suggestions for further modifications to the sputter system . 129

5.3 Summary of results of $\mathrm{ZnO}$ deposition process . . . . . . . . 130

5.4 A simple model for $\mathrm{ZnO}$ on glass deposition . . . . . . . . . 131

5.5 An optimum deposition process . . . . . . . . . . . . . . 133

5.6 Suggestions for future work . . . . . . . . . . . . . . . 134 


\section{List of Figures}

2.1 Growth in the number of $\mathrm{ZnO}$ publications over the last few decades as reported by the SCOPUS data base [30]. . . . . . 8

2.2 The $\mathrm{ZnO}$ wurtzite lattice placed within a hexagonal prism. Grey and black spheres indicate $\mathrm{Zn}$ and $\mathrm{O}$ atoms respectively. The green lines show the primitive cell, where $a$ and $c$ are the lattice constants [48] . . . . . . . . . . . . . . 9

2.3 Some low indexed surface planes present from a single hexagonal crystal. . . . . . . . . . . . . . . . . . . 10

2.4 The piezoelectric effect explained using a simple molecular model. . . . . . . . . . . . . . . . . . . . 11

2.5 A simple single element transducer structure . . . . . . . . . 15

2.6 Basic growth modes of epitaxial films. . . . . . . . . . . . 17

2.7 Structured zone model of sputtered metal films [67] . . . . . 19

2.8 Deposited films under compressive and tensile stress. . . . . 21

2.9 Simplified schematic of a DC sputtering system. . . . . . . . 23

2.10 Interaction of ions at the target surface. . . . . . . . . . . 24

2.11 RF magnetron sputtering system. . . . . . . . . . . 26

3.1 Block diagram of the sputter coater vacuum system. . . . . . 30

3.2 (a) Components inside the Auto500 sputter coater chamber, and (b) The complete sputtering system with the interfacing control panel on the right and deposition chamber on the left. 31

3.3 Annealing furnace housing the quartz tube. . . . . . . . . . 36 
3.4 Temperature profile through the quartz tube inside the furnace showing approximate position of the ceramic boat inside the furnace. . . . . . . . . . . . . . . 36

3.5 Schematic of Dektak surface profilometer used in measuring film thickness. . . . . . . . . . . . . . . 37

3.6 Simplified schematic of UV Spectrophotometer. . . . . . . . 38

3.7 Transmittance spectra for a $\mathrm{ZnO}$ film. . . . . . . . . . . . . . 39

3.8 Absorption coefficient as a function of photon energy at thickness $=0.55 \mathrm{um}$. The bandgap is estimated to be $\approx 3.24 \mathrm{eV} \ldots 41$

3.9 X-rays colliding with atoms in a crystal lattice. . . . . . . . . 43

3.10 XRD spectra from powdered $\mathrm{ZnO}$ [95] . . . . . . . . . . . . 44

3.11 Demonstrating a shift in the $2 \theta$ peak position relative to a stress and strain seen by the lattice. . . . . . . . . . 46

4.1 Deposition chamber pressure as a function of Ar gas flow as measured by our system and as stated in the HHV Auto500 manufacturer's manual. The black dotted line indicates the typical Ar flow, and associated working pressure that was used during most of our initial depositions. . . . . . . . . . . 54

4.2 Variation in deposition rate as a function of the RF power, film thickness was determined by the thickness monitor inside the chamber. The black dotted line indicates the typical RF power used in later depositions. . . . . . . . . . . . 56

4.3 Variation in deposition rate as a function of Argon flow over a range of RF powers. All other parameters held constant: RF power $=180-360 \mathrm{~W}$, chamber pressure $=3 \times 10^{-3} \mathrm{mbar}$

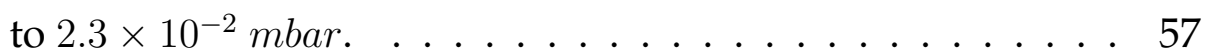

4.4 Substrate temperature increases linearly with increasing RF power over the range of $200 \mathrm{~W}$ to $320 \mathrm{~W}$. . . . . . . . . 58

4.5 XRD spectrum of one of the first $\mathrm{ZnO}$ films deposited where the position of the peak suggests the (002) reflection present in $\mathrm{ZnO} \ldots \ldots \ldots$. . . . . . . . . . . . . . . . . 60 
$4.6 \mathrm{UV}$-visible transmission spectrum of a $\mathrm{ZnO}$ film. . . . . . . . 60

4.7 Estimating the bandgap from a plot of the absorption coefficient vs. photon energy, of which was $\approx 3.27 \mathrm{eV}$. . . . . . 61

4.8 Shows the transparency of a $\mathrm{ZnO}$ deposited film. . . . . . . . 61

4.9 XRD spectra as a function of RF power. There is degradation of the (002) peak with increasing RF power. All other parameters held constant: $\mathrm{Ar}=5 \mathrm{sccm}$, substrate holder stationary and sputtering time $=40$ mins. . . . . . . . . 62

$4.10 \mathrm{XRD}$ spectra for various argon flows. All other parameters held constant: RF power $=180 \mathrm{~W}$, substrate holder rotating, at Ar flow $=3.5,5$ and $10 \mathrm{sccm}$ chamber pressures were $1.3 \times$ $10^{-2}, 2.5 \times 10^{-2}$ and $6.0 \times 10^{-2}$ mbar respectively. . . . . . .

4.11 Deposition rates as a function of the three Ar gas flows. The trend observed is similar to that seen in Section 4.1 suggesting that deposition rate is the highest between $\approx 4-6 \mathrm{sccm}$.

4.12 The influence of substrate heating on crystal structure. All other parameters held constant: $\mathrm{RF}$ power $=180 \mathrm{~W}$, Ar $=$ $6 \mathrm{sccm}$, substrate holder rotating, and substrate temperature at ambient $37^{\circ} \mathrm{C}$ and at $100^{\circ} \mathrm{C}$ with heating. . . . . . . . .

4.13 Configuration of the substrates with reference to the $\mathrm{ZnO}$ target, an important characteristic during the stationary holder deposition due to an expected higher deposition rate on films closer to the target. . . . . . . . . . . . . . 67

4.14 Distribution of growth rates observed across two depositions with eight slides used as substrates in each deposition. In (a) The substrate holder was rotated at $35 \mathrm{rpm}$, while in (b) The substrate holder was stationary. . . . . . . . . . . 68

4.15 Crystal quality results at deposition rates of $\approx 1.1$ and $\approx$ $1.4 \mathrm{~nm} / \mathrm{min}$ with a rotating substrate holder. All other parameters held constant: $\mathrm{RF}$ power $=180 \mathrm{~W}, \mathrm{Ar}=5 \mathrm{sccm}$, and sputtering time $=60$ mins. . . . . . . . . . . 69 
4.16 XRD crystal quality as a function of deposition rate with a stationary substrate holder: (a) Slide \#2, (b) Slide \#3 and (c) Slide \#4. Measurements were performed at $\approx 40,75$ and $105 \mathrm{~mm}$ from the centre of the substrate holder. All other parameters held constant: RF power $=180 \mathrm{~W}, \mathrm{Ar}=5 \mathrm{sccm}$, and sputtering time $=60$ mins. . . . . . . . . . 70

4.17 The (002) FWHM for slides 2, 3 and 4 from the stationary holder deposition as a function of deposition rate. . . . . . 71

4.18 Inside the sputter coater chamber with the substrate holder attached to the shaft at a new substrate-to-target distance of $75 \mathrm{~mm}$. Aluminium foil is covering the quartz lamp to prevent deposited layers building up on the surface. . . . . 73

4.19 Influence of substrate-to-target distance on deposition rate for a rotating substrate holder. All other parameters held constant: RF power $=240 \mathrm{~W}, \mathrm{Ar}=6 \mathrm{sccm}$, no external heating and sputtering time $=60$ mins. . . . . . . . . . 74

4.20 The configuration of the three samples on substrate holder with respect to $\mathrm{ZnO}$ target position. . . . . . . . . . 75

4.21 Comparing deposition rates between a stationary and rotating substrate holder. All other parameters held constant: $\mathrm{RF}$ power $=240 \mathrm{~W}, \mathrm{Ar}=6 \mathrm{sccm}$, no external heating and sputtering time $=60$ mins. . . . . . . . . . 76

4.22 Crystal quality as a function of deposition rate with a stationary substrate holder: (a) Slide \#1, (b) Slide \#2 and (c) Slide \#3. All other parameters held constant: RF power $=$ $240 \mathrm{~W}, \mathrm{Ar}=6 \mathrm{sccm}$, no external heating and sputtering time $=60$ mins $\ldots \ldots \ldots \ldots \ldots \ldots \ldots$

4.23 The ratio between the (002) and (100) peak intensities as a function of deposition rate. As the deposition rate increases past $\approx 15 \mathrm{~nm} / \mathrm{min}$ the film is no longer (001) preferentially oriented. . . . . . . . . . . . . . 78 
4.24 Influence of substrate temperature on crystal quality: (a) Grown at ambient temperature and (b) Grown at $150^{\circ} \mathrm{C}$. All other parameters held constant: $\mathrm{RF}$ power $=240 \mathrm{~W}, \mathrm{Ar}=$ $6 \mathrm{sccm}$ and sputtering time $=60$ mins. . . . . . . . . 80

4.25 Influence of RF power on $\mathrm{ZnO}$ crystal quality at (a) $150 \mathrm{~W}$ and (b) $240 \mathrm{~W}$. All other parameters held constant: Ar = $6 \mathrm{sccm}$, no external heating and sputtering time $=60$ mins. .

4.26 At an RF power of $150 \mathrm{~W}$ the FWHM increases with increasing deposition rate. . . . . . . . . . . .

4.27 2-step deposition: depositing a thin, (001) oriented $\mathrm{ZnO}$ layer to promote epitaxial growth for a faster deposited $\mathrm{ZnO}$ layer. All other parameters held constant: RF power $150 \mathrm{~W}$ for $20 \mathrm{~min}$ followed by $240 \mathrm{~W}$ for $60 \mathrm{~min}$ and $\mathrm{Ar}=6 \mathrm{sccm}$. .

4.28 An (001) oriented film: (a) Surface morphology image, and (b) Cross sectional image. . . . . . . . . . . . . . 86

4.29 A polycrystalline film: (a) Surface morphology image, and (b) Cross sectional image. . . . . . . . . . . . .

4.30 An (001) oriented film grown using the two-step deposition process (a) Surface morphology image, and (b) Cross sectional image. . . . . . . . . . . . . . .

4.31 Influence of 2-step deposition on crystal quality with substrate temperatures at (a) Ambient, and (b) $150^{\circ} \mathrm{C}$. All other parameters held constant: RF power $150 \mathrm{~W}$ for 20 mins then $240 \mathrm{~W}, \mathrm{Ar}=6 \mathrm{sccm}$ and sputter time $=60$ mins. . . . . . . . 90

4.32 Images of cracked $\mathrm{ZnO}$ films: (a) An SEM image of a crack through a $\mathrm{ZnO}$ film, (b) An SEM image of a progressively larger crack, (c) An SEM image of a cracked film which has also peeled in some regions, (d) The initial cracking and peeling on the layer, observed on the region of the ring. The dark image in the photo is the reflection of the camera. . . . 
4.33 Cross sectional images of delaminated $\mathrm{ZnO}$ films: (a) $\mathrm{ZnO}$ film peeling from the substrate surface, and (b) A flake of $\mathrm{ZnO}$ resting on the substrate surface. The bowing direction of these films indicates that they were compressively stressed. 95

4.34 Calculated stress within the films as a function of deposition rate at various RF powers. (a) $150 \mathrm{~W}$, (b) $240 \mathrm{~W}$, (c) and (d) Two-step depositions $150 \mathrm{~W}$ for $20 \mathrm{~min}$ followed by $240 \mathrm{~W}$ for $60 \mathrm{~min}$. The dotted line indicates the observed stress at $10 \mathrm{~nm} / \min . \ldots \ldots \ldots . \ldots 102$

4.35 For various depositions, the graph shows the thickest regions on the films which had not peeled. This suggests that films deposited above $\approx 1.2 \times 10^{-2}$ did not peel. . . . . . . . 104

4.36 Initial annealing test performed on a hot plate at $400^{\circ} \mathrm{C}$ for 1 hour in air. (a) A film with (001) orientation prior to annealing, and (b) A polycrystalline film prior to annealing. . . 106

4.37 Crystal quality and surface morphology for annealed $\mathrm{ZnO}$ films: (a) Surface morphology of an as-sputtered film, (b) XRD spectra of the film as-sputtered and annealed at $250^{\circ} \mathrm{C}$, (c) Surface morphology after annealing at $250^{\circ} \mathrm{C}$, (d) XRD spectra of the film as-sputtered and annealed at $400^{\circ} \mathrm{C}$, (e) Surface morphology after annealing at $400^{\circ} C$, (f) XRD spectra of the film as-sputtered and annealed at $600^{\circ} \mathrm{C}$, and $(\mathrm{g})$ Surface morphology after annealing at $600^{\circ} \mathrm{C} \ldots \ldots$. . . . 108

4.38 (a) and (b) Show surface morphology images of the annealed film at $750^{\circ} \mathrm{C}$, and (c) Compares the XRD spectra of the assputtered film and after annealing at $750^{\circ} \mathrm{C} \ldots \ldots . . . .110$ 
4.39 Surface morphology and XRD spectra of $\mathrm{ZnO}$ film annealed at $900^{\circ} \mathrm{C}$ : (a) Low magnification image of the films surface, (b) A close up image of the 'rounded' patches, Figures (c) and (d) Show high magnification images of circled areas 1 and 2 from Figure b respectively, (e) XRD spectra of the assputtered film and after annealing at $900^{\circ} \mathrm{C} \ldots \ldots$. . . . . . 111

4.40 Comparing the effects of annealing temperatures on (a) The FWHM, and (b) The calculated stress. . . . . . . . . . . . . 113

4.41 Demonstrating deposition rates and film uniformity across the substrates. (a) Shows the positions of the substrates with respect to the $\mathrm{ZnO}$ target and measured thicknesses, and (b) Expresses the thickness data in a surface plot. . . . . . . . . . 116

4.42 Inside the sputter coater: shows the new substrate holder attached to the shaft extension, substrate-to-target distance $75 \mathrm{~mm}$. The substrate heater is mounted to a bracket above the substrate holder. . . . . . . . . . . . . . . . . 117

4.43 Substrate configuration using substrate holder \#2. . . . . . . 118

4.44 Demonstrating the film uniformity across the substrates using the second substrate holder. (a) Shows the positions of the substrates with respect to the $\mathrm{ZnO}$ target and measured thicknesses, and (b) Expresses the thickness data in a surface plot. . . . . . . . . . . . . . . . . 119

4.45 Comparing $\mathrm{ZnO}$ film colour between depositing with the (a) Old $\mathrm{ZnO}$ target appears clear in colour, and (b) New $\mathrm{ZnO}$ target appears brownish in colour. Shows the glass substrate with the deposited $\mathrm{ZnO}$ film to the right. . . . . . . 121

4.46 XRD comparison between the old $\mathrm{ZnO}$ target and the new $\mathrm{ZnO}$ target. The deposition rate for both films was $\approx 15 \mathrm{~nm} / \mathrm{min} .122$

4.47 Comparing the UV-visible transmission for the various $\mathrm{Ar}: \mathrm{O}_{2}$ ratios: (a) 100:0, (b) 87.5:12.5, (c) 75:25, and (d) 62.5:37.5 . . . 123 
4.48 Comparing $\mathrm{ZnO}$ film colour when depositing at $\mathrm{Ar}: \mathrm{O}_{2}$ ratios (a) 100:0, (b) 87.5:12.5, (c) 75:25, and 62.5:37.5. Shows the glass substrate with the deposited $\mathrm{ZnO}$ film to the right. 124

4.49 Influence of $\mathrm{Ar}: \mathrm{O}_{2}$ ratios on crystal quality. All other parameters held constant: RF power $=150 \mathrm{~W}$ and sputtering time of 20 mins. . . . . . . . . . . . . . . . . . . . . 125

5.1 Illustrating the nucleation and growth stages (a) A deposition performed at a low deposition rate, films preferentially grow with the c-axis perpendicular to the substrate in the (001), and (b) A deposition performed at a higher deposition rate, resulting in random orientation. Where the blue arrow indicates the c-axis. . . . . . . . . . . . . . 132 


\section{List of Tables}

2.1 Properties of popular piezoelectric materials (data taken from [15], [53] and [54]). . . . . . . . . . . . . . . . . . . 14

3.1 The $2 \theta, \mathrm{d}$-spacing and lattice planes present of a standard reference $\mathrm{ZnO}$ powder. Here $a=3.24982 \AA$ and $c=5.20661 \AA$ [95]. . . . . . . . . . . . . . . . . . . 44 44

3.2 Elastic constants of $\mathrm{ZnO}$ as measured by $[97,98] \ldots \ldots$. . . 47

4.1 Thermal stress induced by soda lime glass and borosilicate at various substrate temperatures. CTE obtained for $\mathrm{ZnO}$ [58], soda-lime glass [107] and borosilicate [89]. . . . . . . . 98

4.2 The estimated stress value at $\approx 10 \mathrm{~nm} / \mathrm{min}$ for the various RF powers. . . . . . . . . . . . . . . . . . . 101

4.3 Summarising the main XRD results from the as-sputtered films and after annealing. . . . . . . . . . . . . . . 109 


\section{Chapter 1}

\section{Introduction}

The aim of this thesis was to develop a deposition process using the RF magnetron sputtering technique to deposit piezoelectric $\mathrm{ZnO}$ thin films for the application in ultrasound transducers. In particular, it will focus on developing a process for c-axis oriented films, as this is the first requirement for piezoelectric activity in $\mathrm{ZnO}$. At the same time it will aim to achieve such a microstructure at high deposition rates, so as to enable practical device fabrication.

Piezoelectricity is the phenomenon through which certain materials convert an applied mechanical stress into an electric charge. The converse effect is also found in the same materials, where the application of a voltage to the material causes a physical deformation. This phenomenon has been the basis for many electronic devices, including ultrasound transducers [1-5], bulk acoustic wave (BAW) and surface acoustic wave (SAW) resonators [6,7], piezoelectric sensors [8] and vibration energy harvesting devices $[9,10]$.

The piezoelectric effect was first discovered by brothers Pierre and Jacques Curie in 1880 [11], but more than 30 years passed before it was used in a practical application [12]. During World War I a means of submarine detection was of great interest. Langevin, a French physicist, proposed the design of a device utilizing the piezoelectric effect to detect the pres- 
ence of submarines, submarine mines, sand banks and icebergs [13]. His device, an ultrasound transducer, used naturally occurring quartz crystal as the active piezoelectric element to transform an oscillating voltage into elastic waves of the same frequency and vice versa. These elastic waves would propagate through the water and the reflected echo could then be used to determine the presence of an obstacle. Shortly after, in 1918, a second application was demonstrated by Nicholson who used Rochelle salt to construct a crystal oscillator [14]. Today these naturally occurring piezoelectric crystals used in early device fabrication have been totally replaced with artificially produced materials with bulk single crystal quartz used in crystal resonators and lead zirconate titanate (PZT) or related ceramic materials the material of choice in the production of ultrasound transducers.

PZT, a ferroelectric ceramic, has become the favoured material for ultrasound transducers due to its high piezoelectric coefficient of $117 p C N^{-1}$ $[10,15,16]$. The material is typically produced as polycrystalline ingots, poled, and cut into wafers for device processing [2]. For use in ultrasound transducers the PZT wafer is thinned to the desired thickness, as the thickness of the piezoelectric material governs the operating frequency of the transducer. Such devices are extensively used for nondestructive testing and ultrasonic imaging in the medical industry $[5,16,17]$. In these applications the transducer, in conjunction with signal processing techniques, creates an image of the internal elastic structure of the material under test. Both the resolution and image depth is determined by the operating frequency of the transducer. As with all engineering systems there is a tradeoff between functionalities. At lower operating frequencies $(<10 \mathrm{M} \mathrm{Hz})$ the ultrasonic wave can penetrate deep into the material, allowing for greater imaging depth, but at a cost of lower spatial resolution. At higher operating frequencies ( $>50 \mathrm{MHz}$ ) there is a shallower imaging depth, but imaging of smaller features is possible.

Very high frequency transducers $(>100 \mathrm{MHz}$ ) are desired in acoustic microscopy to image sub-micron features $[3,5]$. There is thus a drive to 
produce higher frequency transducers which places a limit on the use of bulk ceramic materials such as PZT for this application. This is due to the fact that the ceramic will become mechanically brittle when thinned and may also shatter under excess voltage. For this reason it becomes impractical to produce very high frequency transducers from bulk ceramics. An alternative fabrication method is to deposit a thin piezoelectric film by either a vacuum or solution process onto a solid substrate $[16,18]$. A number of different materials are under consideration for thin film piezoelectrics [16]. These include PZT deposited by modified sol-gel solution [19], aluminium nitride (AlN) and $\mathrm{ZnO}$ deposited by DC and RF sputtering [20].

$\mathrm{ZnO}$ is a group II-VI compound semiconductor attainable in bulk, thin film and nanostructure forms, making it a versatile material to work with. It encompasses a unique combination of electronic and mechanical properties making it an attractive material in many device applications. It has a direct, wide band gap $(\approx 3.4 \mathrm{eV}[21])$ and high exciton energy $(\approx 60 \mathrm{meV}$ [22]) making it ideal in optoelectronic applications, such as LEDs, photodiodes and laser diodes [23]. It has become a popular material in developing novel nanostructure devices, including solar cells, biosensors and gas sensors [24-26]. It is also a promising candidate for use as the thin film piezoelectric material in ultrasound transducers due to its relatively high piezoelectric coefficient and relative ease of thin film deposition [3-5, 19]. ZnO can be deposited using a number of chemical and physical techniques, including chemical vapour deposition (CVD), sol-gel synthesis, molecular beam epitaxy (MBE), pulse laser deposition (PLD) and sputter deposition $[21,27,28]$.

The aim of this project was to develop a deposition procedure using the RF magnetron sputtering technique for the deposition of thin $\mathrm{ZnO}$ films with suitable properties for piezoelectric device fabrication. In the summer of 2011 Victoria University of Wellington (VUW) was equipped with a new sputter deposition system. Sputtering techniques have been a proven method in producing high quality c-axis, piezoelectric $\mathrm{ZnO}$ films $[1,4,29]$ 
and for these reasons was the technique used in this research. A first requirement for the use of $\mathrm{ZnO}$ thin films in piezoelectric devices is to optimise the piezoelectric properties of the film. In the case of using $\mathrm{ZnO}$ in ultrasound transducer elements, it means that the film needs to be oriented with the crystallographic c-axis perpendicular to the substrate surface. To achieve this, the project was broken down into the following steps:

1. Evaluate the new sputter coater system for $\mathrm{ZnO}$ deposition and make changes where needed.

2. Determine how the deposition parameters influence the $\mathrm{ZnO}$ films material properties, including: crystalline structure, surface morphology and optical properties. Select optimum deposition parameters to grow uniform, c-axis oriented films, ideally at deposition rates $\geq 1 \mu \mathrm{m} /$ hour.

3. Gain an understanding of the thin film deposition process on amorphous (glass) substrate.

The remainder of this thesis comprises of five chapters:

Chapter 2 discusses the background theory of the topics covered in this project. The first section introduces $\mathrm{ZnO}$ as an electronic material with section two briefly discussing piezoelectricity and the application of $\mathrm{ZnO}$ in piezoelectric ultrasound transducers. Section three describes the growth modes of epitaxial film growth leading into section four which provides a brief analysis of thin film deposition techniques, and the sputter deposition method. In the last section the general properties of $\mathrm{ZnO}$ thin films to be used in ultrasound transducers are defined.

Chapter 3 describes the experimental and film characterisation techniques used during this project. The chapter begins by describing the sputter coater system used for depositing $\mathrm{ZnO}$. After a deposition the films could be characterised by their thickness, optical transmission in the visible region, crystallographic structure and surface morphology, the techniques of which are described in this chapter. 
Chapter 4 presents the results of the project. Firstly an initial evaluation is performed on the sputter coater system and modifications made to improve functionally specific for the project. It then discusses the influence of the deposition parameters on the deposition rate, crystalline structure and surface morphology of the $\mathrm{ZnO}$ films. An analysis of film stress is performed with post growth annealing investigated as a technique to relax film stress. A further design modification to improve film uniformity is described and results are concluded by showing the potential problems from variations in sputter targets.

Chapter 5 provides a summary and the conclusions of the project. It also proposes a simple model for the deposition of $\mathrm{ZnO}$ on glass substrates as well as an optimum four-step deposition process. Future work in the development of devices from $\mathrm{ZnO}$ is also presented.

Overall the thesis presents a guide for the successful fabrication of (001) oriented $\mathrm{ZnO}$ thin films deposited using the RF magnetron sputtering technique on amorphous substrates. The requirement of c-axis film orientation at high deposition rates is found to be a trade-off, and a deposition procedure is developed to satisfy both these requirements. The important factor of inherent stress development in these films is pointed out and procedures developed to minimise stress. The process developed here can be used for the production of $\mathrm{ZnO}$ films for piezoelectric applications. 


\section{Chapter 2}

\section{Background Theory}

\subsection{History and properties of $\mathrm{ZnO}$}

In this chapter the material properties of $\mathrm{ZnO}$ are described. Piezoelectricity is discussed and reference made to the piezoelectric properties of $\mathrm{ZnO}$ and its use in piezoelectric applications. The development of functional thin films are described by the relevant growth models, which leads into the deposition techniques used in growing $\mathrm{ZnO}$ thin films. A brief discussion on the theory of sputter deposition is described and reports on successful (001) oriented growth of $\mathrm{ZnO}$ using the sputtering deposition technique presented.

The use of $\mathrm{ZnO}$ dates back to the Bronze Age, where the compound originated as a by-product of copper smelting and was used as an ointment to heal wounds [30]. It became widely used by the Romans, as early as $200 B C$, to produce the first copper-zinc alloy, brass [31]. Modern applications for $\mathrm{ZnO}$ were found in the mid 1800s by the cosmetics and paint industries as a nontoxic alternative to lead based components $[32,33]$. The 1920s saw ZnOs debut in electronic applications where it was used as a cat-whisker detector in early radio sets [34]. The detector was constructed from a $\mathrm{ZnO}$ crystal and thin copper wire which converted the incoming alternating current $(\mathrm{AC})$ radio waves into a direct current (DC) signal. 
With the early $20^{\text {th }}$ century came the birth of modern material science, an era where fundamental properties of materials would be discovered. In 1935 the lattice parameters of $\mathrm{ZnO}$ were determined [35] and in 1954 its optical properties were studied [36]. Shortly after, in 1960, the piezoelectric properties were discovered [37] and put to application in 1976 to produce a surface wave transducer [38]. In the 1970s and 1980s ZnO continued to be of interest. However, from the mid 1990s onwards, there has been a major increase in $\mathrm{ZnO}$ interest, as shown by the $\mathrm{ZnO}$ publications shown in Figure 2.1. The renewed interest in $\mathrm{ZnO}$ stems from reports of ferromagnetic properties [39-41], the search for stable p-type doping [42, 43], the advancement of nanostructure fabrication $[25,44]$ and its potential use in thin-film transistors $[45,46]$. Recent reviews on $\mathrm{ZnO}$ have been by published by Ozgur, Ellmer and Klingshirn et al. [21,30,47], which readers are directed to for further information.

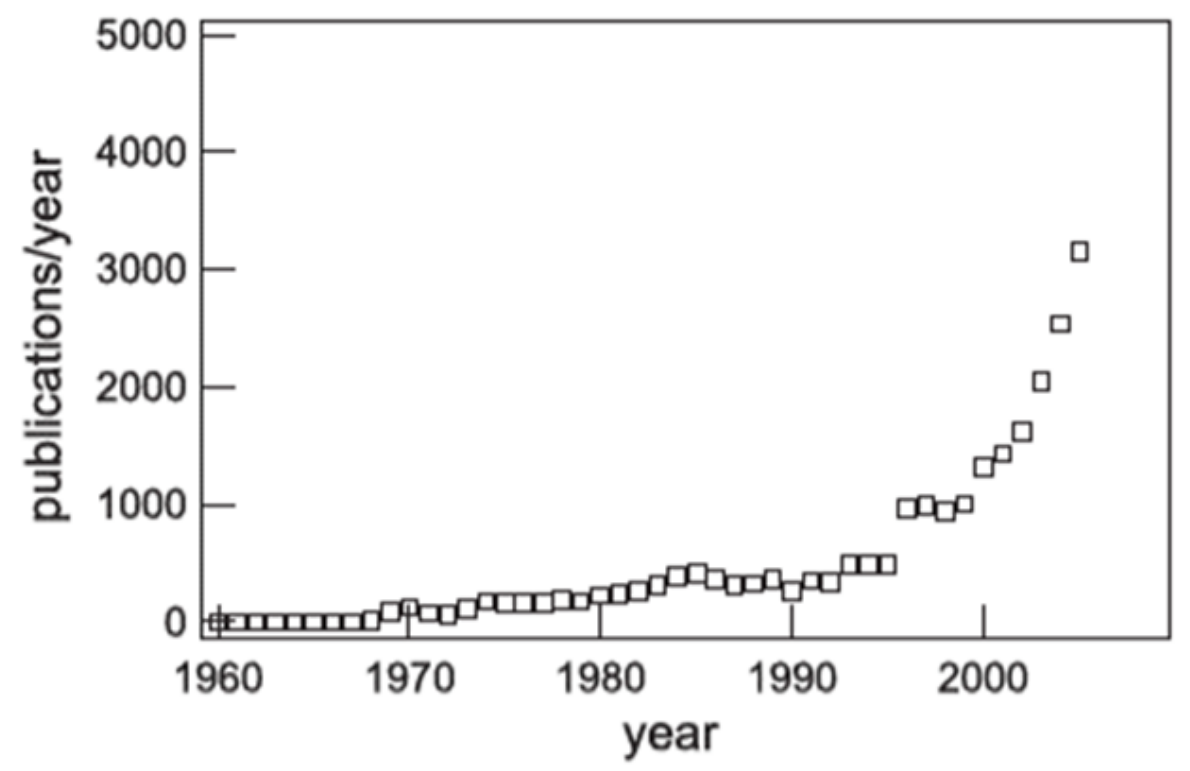

Figure 2.1: Growth in the number of $\mathrm{ZnO}$ publications over the last few decades as reported by the SCOPUS data base [30]. 


\subsubsection{Crystal Structure}

$\mathrm{ZnO}$ is a II-VI compound semiconductor, and can take on three possible crystal structures: hexagonal wurtzite, cubic zinc blende and cubic rocksalt [21]. Each of these structures are formed under unique deposition conditions. By far the most common structure is hexagonal wurtzite, which is thermodynamically stable and formed under ambient conditions [48]. The zinc blende structure can occur when $\mathrm{ZnO}$ is deposited onto a cubic substrate, while the rocksalt structure is formed under pressures in excess of $9 \mathrm{GPa}$ [21]. For this research we are only interested in the wurtzite structure because it shows a larger piezoelectric response than the zinc blende structure [49].

The wurtzite structure has a hexagonal unit cell belonging to the space group $\mathrm{P}_{3} m c$ [50]. The unit cell can be described by three lattice parameters $a, b$ and $c$, as shown in Figure 2.2, with $a=b$ in this case. These parameters have been reported in the literature as $a=3.2475-3.2860 \AA$ and $c=5.2042-5.2410 \AA$ [21].

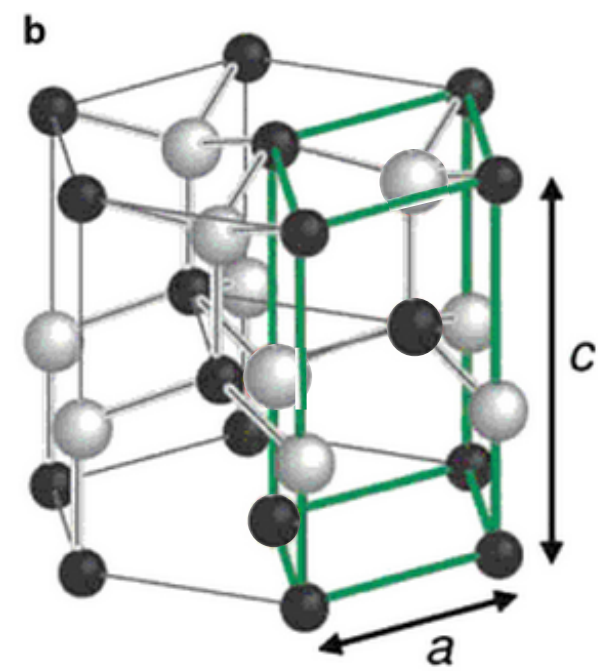

Figure 2.2: The $\mathrm{ZnO}$ wurtzite lattice placed within a hexagonal prism. Grey and black spheres indicate $\mathrm{Zn}$ and $\mathrm{O}$ atoms respectively. The green lines show the primitive cell, where $a$ and $c$ are the lattice constants [48]. 
For the hexagonal structure the crystal planes can be expressed using the Miller-Bravais indices ( $h k i l$ ). This four-index system can be reduced to the conventional three-index $(h k l)$ system through the symmetry $h+k=$ $-i$. A schematic of the hexagonal structure, indicating some low index planes (which were sometimes found present in films deposited during this research) is shown in Figure 2.3.

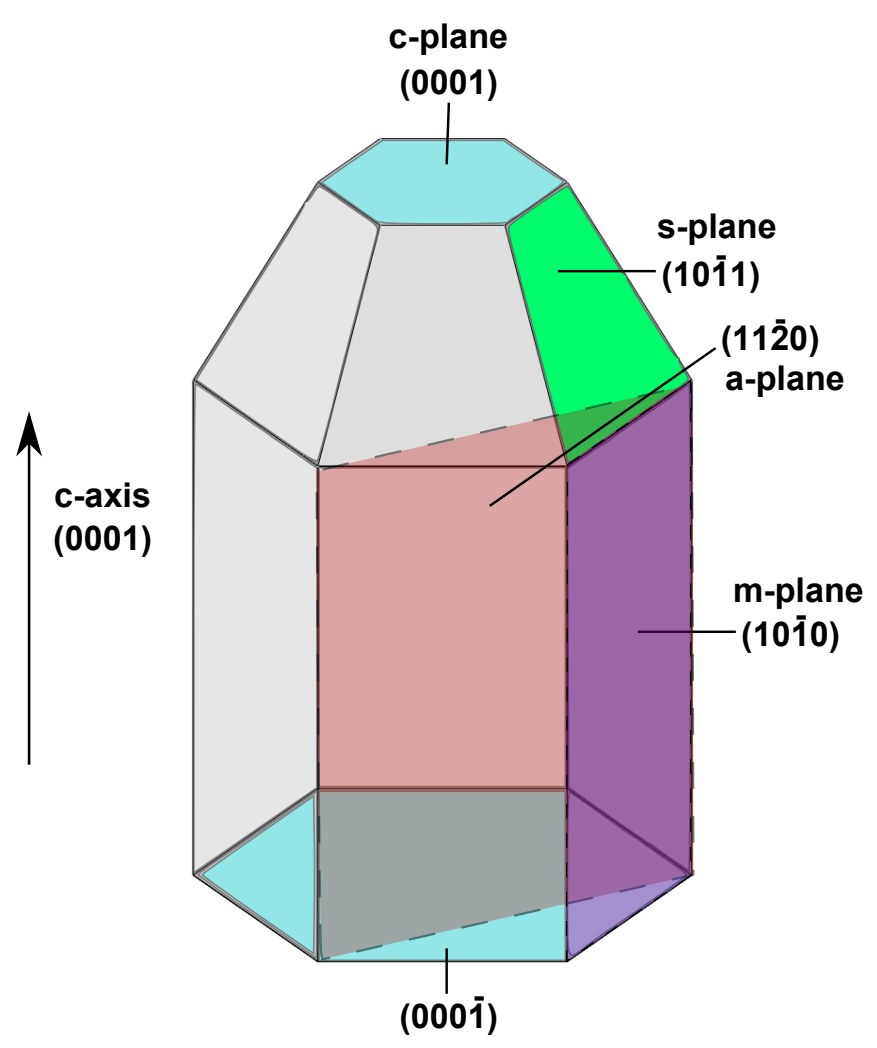

Figure 2.3: Some low indexed surface planes present from a single hexagonal crystal.

The c-plane, running perpendicular to the principle axis is the basal plane. It is non-centrosymmetric having positively charged $\mathrm{Zn}(0001)$ and negatively charged $\mathrm{O}(000 \overline{1})$ surfaces. It is this characteristic of $\mathrm{ZnO}$ that gives rise to a spontaneous polarization resulting in $\mathrm{ZnOs}$ piezoelectric response. 


\subsection{Piezoelectricity}

Piezoelectricity occurs in crystals without a centre of symmetry (non-centrosymmetric). Of the 32 known crystal structures, 20 of them exhibit a piezoelectric response [51]. The basic theory of piezoelectricity can be explained using a simple model consisting of cations (+) and anions (-) and how they move inside a crystal subjected to an external force. When a noncentrosymmetric crystal experiences no external forces, the centre of charge of the cations and anions are balanced, forming a neutral molecule, shown in Figure 2.4a. If an external force is then applied, the crystal structure begins to deform. This causes the separation of the charge centres of the molecules, generating dipole moments, as shown in Figure 2.4b. The dipoles inside the structure sum and cancel, and the material becomes polarised as charge shifts to opposite sides creating an electric field, Figure 2.4c.

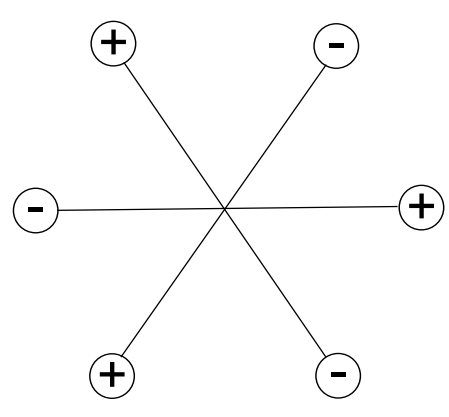

(a) Neutral molecule.

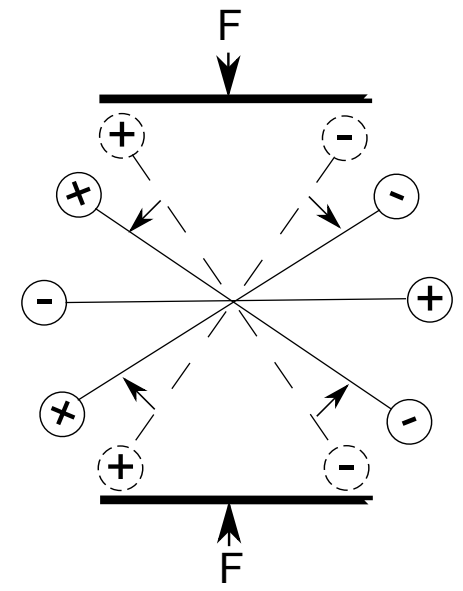

(b) External force applied to molecule.

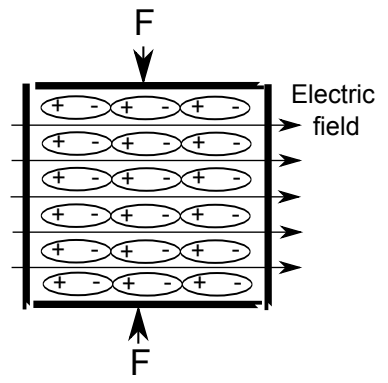

(c) Material becomes polarised

Figure 2.4: The piezoelectric effect explained using a simple molecular model. 


\subsubsection{The linear piezoelectric equations}

Piezoelectric theory relates the applied mechanical stress to the resultant electrical charge. When a solid undergoes compression or expansion the strain $S$ experienced by the solid causes a proportional stress $\sigma$, related by its elastic compliance $s$, which is an indication of the stiffness of the material. This effect is represented by Hooke's Law,

$$
S=s \sigma
$$

When an electric field $\mathrm{E}$ is applied to a dielectric material having no centre of symmetry the bound charges slightly separate. This induces local electric dipole moments within the material. These free charges can be defined by a dielectric displacement $D$,

$$
D=\epsilon E
$$

where $\epsilon$ is the permittivity.

Piezoelectricity is then the combined effect of the mechanical behaviour (as described in Equation 2.1) and electrical behaviour (as described in Equation 2.2).

The direct effect is the generation of electric charge by the application of an applied stress. In terms of the dielectric displacement and stress, this relationship is given by,

$$
D=d \sigma
$$

where $d$ is the piezoelectric coefficient.

For the indirect piezoelectric effect, the application of an applied electric field produces a proportional strain related by,

$$
S=d E
$$

Equations 2.3 and 2.4 have been expressed as linear equations. In reality $D$ and $E$ are first-rank tensors $\left(D_{k}\right.$ and $\left.E_{k}\right)$, and $\sigma$ and $S$ are second-rank 
tensors $\left(\sigma_{i j}\right.$ and $\left.S_{i j}\right)$. The corresponding piezoelectric coefficient $d$ forms a third-rank tensor $\left(d_{k i j}\right)$, where $(i, j, k)=1,2,3$. Using Einstein's summation rule, $d_{k i j}$ can be reduced to the conventional $d_{k m}$. The first subscript indicates the direction of the field, represented as 1,2 or 3 for the $x, y$ and $z$ directions [52]. The second subscript indicates the direction of stress or strain, where $m=1, \ldots, 6$. Compressive or tensile stress is represented by $m=1,2$ or 3 and shear stress by $m=4,5$ or 6 . For example, $d_{k m}=d_{33}$ represents an applied electric field and the applied strain in the $z$ direction. High $d$ coefficients are desirable for materials used in piezoelectric applications.

Another important property of a piezoelectric material is a measure of how well it converts mechanical energy into electrical energy and vice versa. This characteristic is referred to as the electromechanical coupling factor $\kappa$. If an electric field is applied to the material, $\kappa$ measures the effectiveness with which it converts electrical energy into mechanical energy. If the material undergoes a compression or expansion $\kappa$ measures the effectiveness with which it converts mechanical energy into electrical energy. This relationship is given by,

$$
\kappa^{2}=\frac{\text { Electrical energy converted to mechanical energy }}{\text { Input of electrical energy }}
$$

or

$$
\kappa^{2}=\frac{\text { Mechanical energy converted to electrical energy }}{\text { Input of mechanical energy }}
$$

\subsubsection{Electric and piezoelectric properties of $\mathrm{ZnO}$}

Essentially it is the lack of unit symmetry that causes $\mathrm{ZnO}$ to behave as a piezoelectric material. It develops a piezoelectric response along the c-axis, which is obtained with (001) orientation. The magnitude of the response is measured by the piezoelectric coefficient $d_{33}$ and electromechanical coupling coefficient $\kappa$. Typical values for common piezoelectric 
devices are shown in Table 2.1.

\begin{tabular}{|l|l|l|}
\hline Material & $\begin{array}{r}\text { Piezoelectric Coefficient }(d) \\
\left(p C N^{-1}\right)\end{array}$ & $\begin{array}{c}\text { Electromechanical } \\
\text { CouplingCoefficient }(k)\end{array}$ \\
\hline Quartz & $2.3\left(d_{11}\right)$ & 0.1 \\
\hline $\mathrm{AlN}$ & 4.5 & $0.17-0.5$ \\
\hline $\mathrm{ZnO}$ & 12 & $0.15-0.33$ \\
\hline $\mathrm{LiNbO}_{3}$ & $19-27$ & 0.23 \\
\hline $\mathrm{LiTaO}_{3}$ & 21 & 0.2 \\
\hline $\mathrm{PVDF}$ & 35 & $0.12-0.2$ \\
\hline $\mathrm{PZT}$ & 117 & 0.49 \\
\hline
\end{tabular}

Table 2.1: Properties of popular piezoelectric materials (data taken from [15], [53] and [54]).

From Table 2.1, it is clear that $\mathrm{ZnO}$ has a high piezoelectric coefficient but not the highest of the listed materials. It is however a semiconductor making it easy to integrate with standard semiconductor device processing. Also, it has shown itself to be relatively easy to produce thin films by a number of methods, making it the material of choice for this project.

A further advantage of $\mathrm{ZnO}$ is that it has a direct wide bandgap which has been measured in the literature as 3.3 to $3.437 \mathrm{eV}$ at room temperature, making it transparent in the visible spectrum $[55,56]$. It is a good insulator with reports of electrical resistivity $\rho>10^{10} \Omega \mathrm{cm}$ [57], but can be made conductive by intrinsic or extrinsic dopants. It also has a low thermal expansion coefficient $(\perp c)$ equal to $4.75 \times 10^{-6} K^{-1}$ [58] at room temperature. These properties make $\mathrm{ZnO}$ ideal as the active piezoelectric element in ultrasound transducers [3-5]. 


\subsubsection{Applications of $\mathrm{ZnO}$ in piezoelectric devices}

$\mathrm{ZnO}$ has found successful application in many different types of piezoelectric devices, such as ultrasound transducers, BAW and SAW resonators $[6,59,60]$, microelectromechanical system (MEMS) resonators [61] and energy harvesting devices $[9,10]$. This work has been undertaken with ultrasound transducers as the intended application.

Transducers are devices which employ the piezoelectric effect to convert electrical energy into mechanical energy and vice versa. Ultrasound transducers convert electrical energy from an AC signal into an ultrasonic wave and vice versa. A piezoelectric material is at the core of the transducer and is the active element which does the conversions. The piezoelectric element emits ultrasound symmetrically out of both crystal faces, as a result additional layers need to be added to the transducer structure to enhance the acoustic performance. These include a matching layer and lens to couple the energy transmission between the piezoelectric material and load medium, and a backing layer to support the piezoelectric element and dampen out reverberations. Figure 2.5 shows a typical schematic of a transducer.

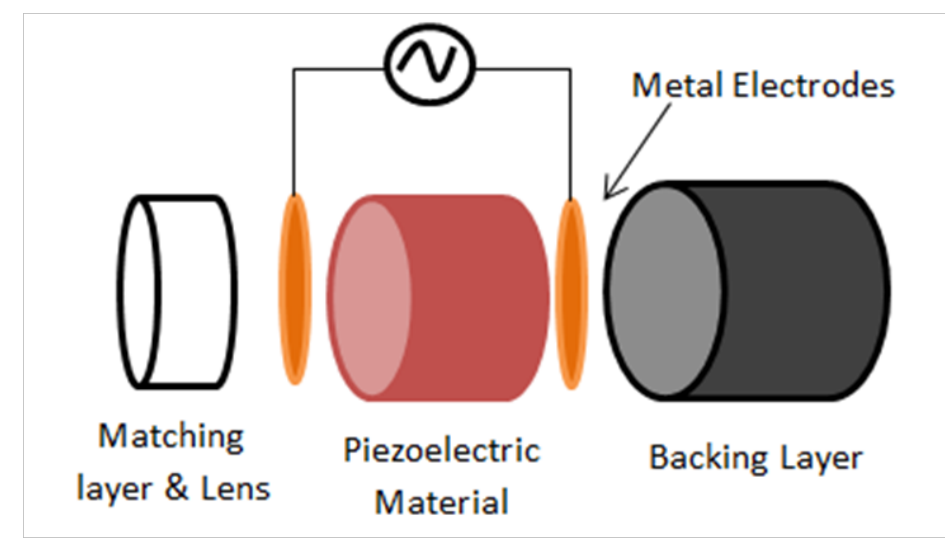

Figure 2.5: A simple single element transducer structure.

Reviews on $\mathrm{ZnO}$ as the active piezoelectric layer in ultrasound transducers have been reported by $[1,3-5,20]$. 


\subsection{Functional thin films}

It is often found that the crystalline structure of thin films differs significantly from that of bulk material due to the role of the substrate and influence of many deposition process parameters. The development of deposition processes that can reproducibly yield thin films of the desired orientation, crystalline perfection and related electrical, optical and mechanical properties has been a central theme in the research and development of new electronic materials. The basis of this research can be traced back to the 1950s when the crystal structure of deposited metal films were studied [62]. The past decades have seen a tremendous development in the technology of thin film deposition processes as well as an increase in the range of materials that can now be deposited. Thin films of compound semiconductors are now routinely deposited with sophisticated techniques such as MBE or organometallic vapour phase epitaxy (OMVPE) [21,28].

The next section provides a brief background on the deposition and structure of thin films, while the following section discusses the sputtering process of thin film formation with reference to $\mathrm{ZnO}$.

\subsubsection{Thin film growth models}

The formation of a film is determined by the nucleation and growth process $[62,63]$. Physical vapour deposition (PVD) is a general term used to describe a variety of vacuum deposition techniques, where thin films are deposited by condensation from the vapour phase to the solid phase. Deposition techniques such as sputtering, MBE and PLD are all examples of PVD. The process of epitaxial film growth by PVD is generally separated into three growth modes:

(i) Layer-by-layer growth (Frank-van der Merwe)

(ii) Island growth (Volmer-Weber) 
(iii) Combination: layer-plus-island growth (Stranski-Krastanov)

The basic theory behind these models is as follows [63]:

Layer-by-Layer: Film growth occurs in two-dimensions as a result of adjacent atoms having a stronger bond to the substrate than to each other. Initially a monolayer of atoms cluster together, inter-growing to form a continuous layer before subsequent layers grow on top, Figure 2.6a.

Island: Film growth occurs in three-dimensions as a result of the bonding between adjacent atoms being stronger than their bond to the substrate. Initially islands of atoms grow to several layers in thickness before they coalesce, Figure $2.6 \mathrm{~b}$

Layer-plus-Island: Is a combination of layer-by-layer and island. For the first several monolayer's layer-by-layer growth occurs until a critical thickness is reached after which island growth begins to dominate, Figure 2.6c.

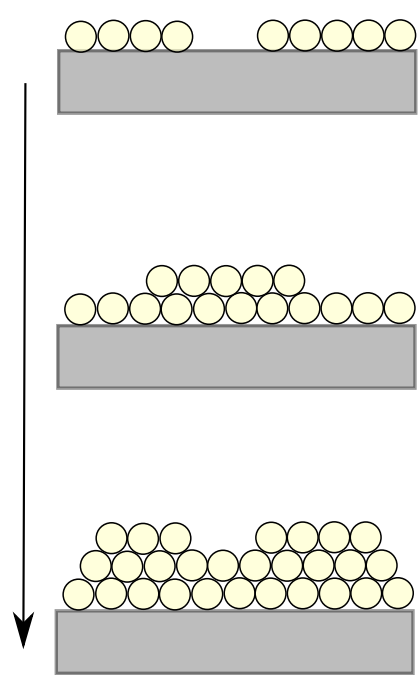

(a) Frank-van der Merwe.
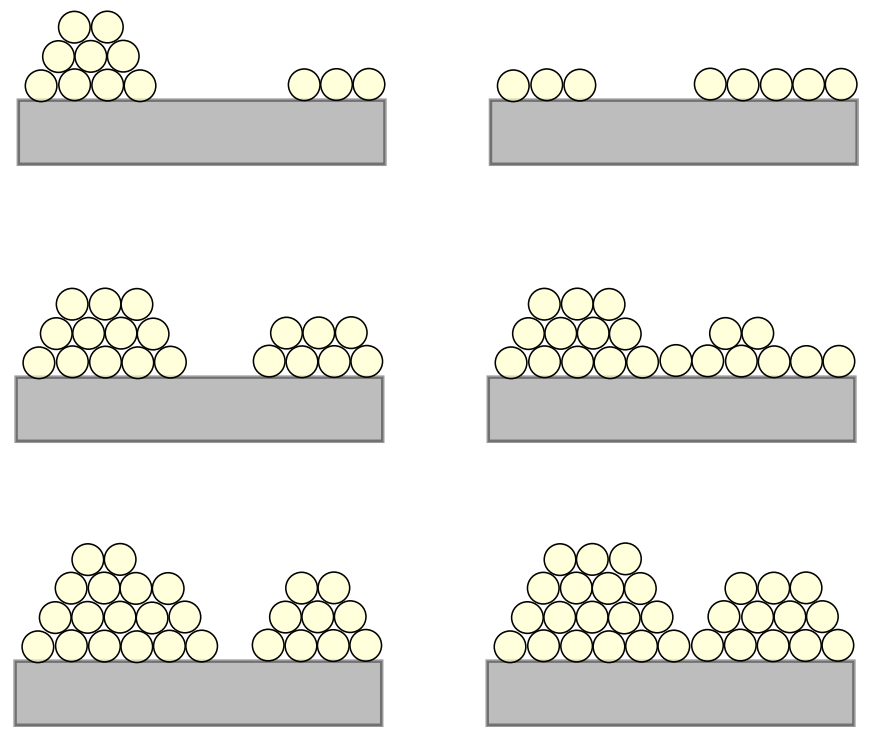

(b) Volmer-Weber
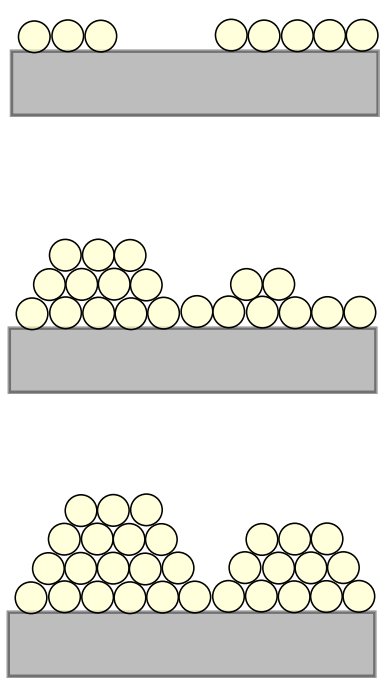

(c) Stranski-Krastanov

Figure 2.6: Basic growth modes of epitaxial films. 
The morphology of the film is influenced by factors such as: the energy of the arriving particles, the nature and pressure of the chamber, and the nature and temperature of the substrate $[64,65]$. By manipulating these parameters films with particular properties can be grown.

\subsubsection{Oriented film growth}

The crystallographic orientation of a film is influenced by several mechanisms. The resultant crystallographic relationship between the thin film and substrate can be determined by a range of factors including the crystalline structure and orientation of the substrate and the process deposition parameters. In general there are three types of substrate-film textures found:

- Epitaxial films: The film has a specific crystallographic orientation with respect to the substrate. This is normally found with a single crystal substrate with good lattice match to the film

- Preferred orientation or textured films: The film consists of grains or crystallites which all have the same orientation, but there is no alignment between individual grains.

- Polycrystalline film: The film consists of grains with multiple orientations.

\subsubsection{Structure zone model (SZM)}

During the 1960s, Movchand and Demchishin studied the surface and cross sectional morphology of evaporated layers of metals and oxides. They proposed a model comprising of three zones to describe the behaviour in film structure morphology over a certain temperature range [66]. This description has come to be known as the structure zone model (SZM) and has been adapted for various deposition techniques. In 1974 
Thornton applied the SZM model to sputtered metal films [67]. The model included the addition of a fourth zone and a second axis introducing the influence of argon pressure. Thornton's extended model is shown in Figure 2.7, where $T$ is the substrate temperature during the deposition and $T_{m}$ is the depositing materials melting point.

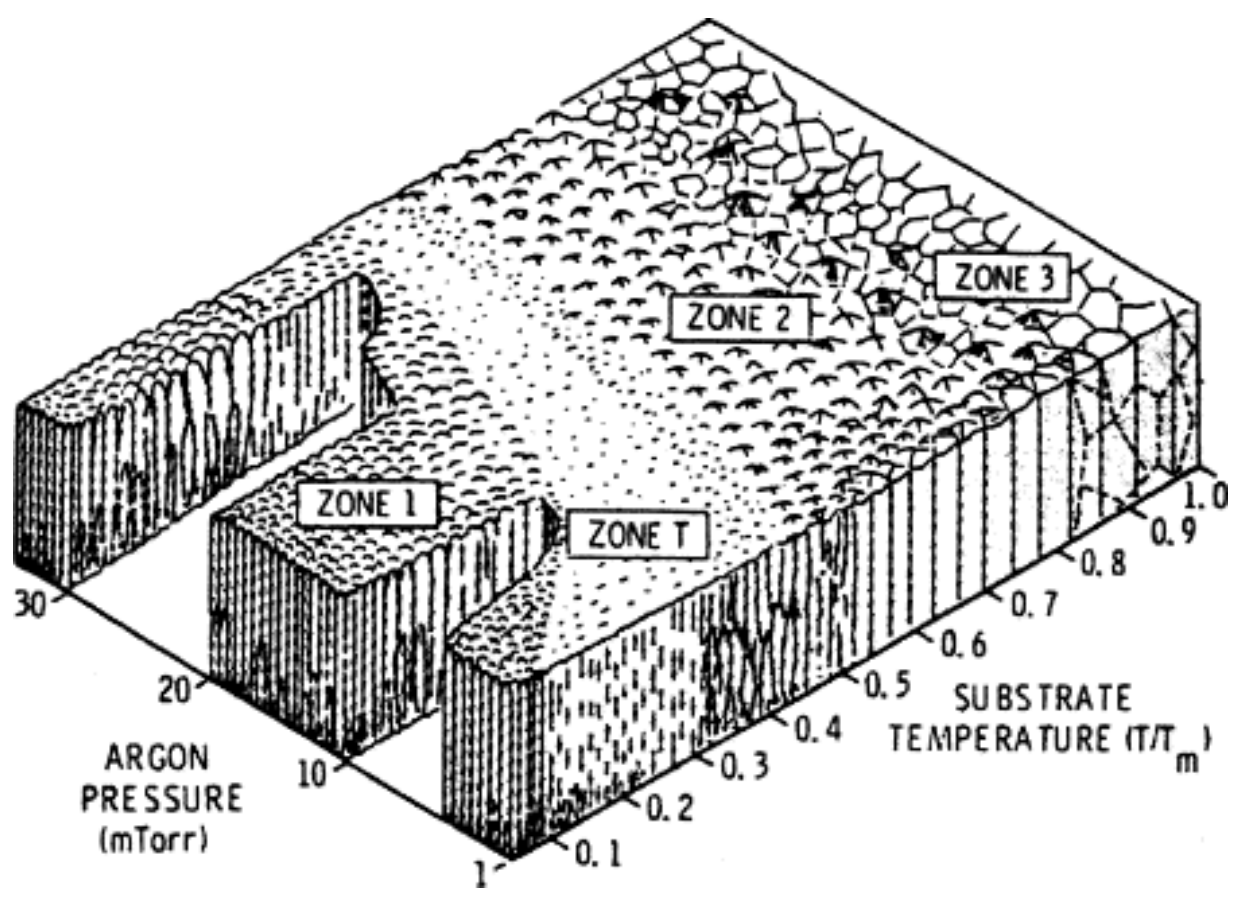

Figure 2.7: Structured zone model of sputtered metal films [67].

Films grown in Zone 1 have low adatom mobility and as a result form tapered columns with a rough surface. If the substrate temperature is increased the film enters the $\mathrm{T}$ Zone, the transitional zone. Films deposited in this region have a smoother surface morphology and weaker grain boundaries. In Zone 2, the adatom mobility is high and growth is controlled by surface diffusion [64]. In this region tall narrow columnar grains are grown forming a dense structure. In Zone 3 bulk diffusion dominates, where recrystallisation forms large, but not necessarily columnar grains.

Thornton's SZM classifies the surface and cross sectional morphology 
of sputtered metal films and as a result cannot be directly related to sputtering $\mathrm{ZnO}$, a semiconducting material. However, it is broadly applied as the model provides some general properties on film structure from the influence of substrate temperature and deposition pressure [64].

\subsubsection{Residual stress in thin films}

In general, all deposited thin films grown on a substrate surface undergo some form of residual stress [68]. Stress within a film can manipulate its structure, affecting the electronic and mechanical properties of the layer. Thin film stress can be classified as either intrinsic or extrinsic. Intrinsic stress is induced during the deposition process and is mainly caused by the microstructure of the thin film. The most common factors influencing the intrinsic stress within a film are the growth technique, grain growth (island nucleation, coalescence and voids), the insertion of excess atoms and impurities $[69,70]$. Extrinsic stress is induced by post-deposition processing or external factors. These include thermal stress induced by the difference in the thermal expansion between the substrate and film, and lattice mismatch between the film and substrate [68].

A $\mathrm{ZnO}$ film under stress will show relaxation over time altering the properties of the film [71]. A large stress may result in the film cracking or the layer peeling off the substrate [72]. The stress within the film creates bending moments between the film-substrate interfaces. The direction of this bend is classified as either compressive or tensile. Compressive stress occurs when the film is compressed to fit the substrate, shown in Figure 2.8a. Tensile stress occurs when the film stretches to fit the substrate, shown in Figure $2.8 \mathrm{~b}$. 


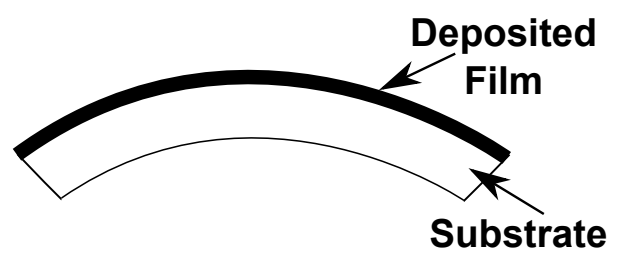

(a) Compressive stress.

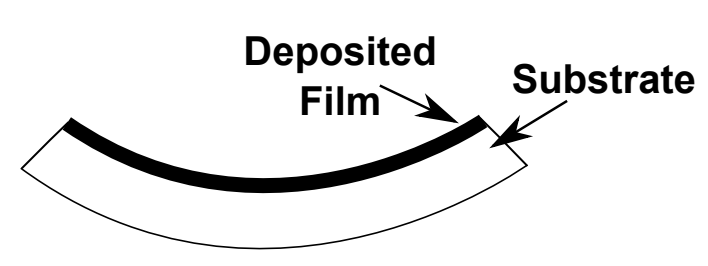

(b) Tensile stress

Figure 2.8: Deposited films under compressive and tensile stress.

\subsection{Depositing $\mathrm{ZnO}$ thin films}

In recent years there has been extensive study on $\mathrm{ZnO}$ deposition processes. Both chemical deposition techniques, such as: CVD and sol-gel synthesis [73] and physical deposition techniques, such as MBE, PLD and sputter deposition have been used to deposit thin films of $\mathrm{ZnO}$. Comparisons of such techniques can be found in reviews by Triboulet and Özgür [21,28].

Of these techniques sputter deposition is a common method; its ability to deposit insulators, semiconductors and conductors make it a versatile technique. It offers advantages such as: deposition at low substrate temperatures (down to room temperature), offers moderately high deposition rates, good thickness uniformity, good controllability of the deposition parameters and the capability to grow c-axis orientated $\mathrm{ZnO}$ films [74]. Sputter deposition is a well understood process and a proven technique used in producing piezoelectric $\mathrm{ZnO}$ films [1, 4,29].

\subsubsection{Sputtering theory}

The sputtering process is well established and has been extensively studied in the literature. What will be described here is an overview of the underlying theory behind the sputtering process. For an in-depth analysis refer to $[30,75,76]$. 
Sputtering is a physical vapour deposition technique. It is a process whereby atoms or molecules are ejected from a target (the material to be deposited) by the bombardment of ions. The technique is described by Johnson using a helpful analogy [77].

"The sputtering process can be compared to throwing steel balls at a concrete wall. As the balls strike the wall, small fragments of concrete tear away from the wall. If the process is continued, eventually the surrounding area will be covered in a thin layer of concrete dust". [As the concrete dust covers the surrounding area, it also covers the substrate.] Where the concrete wall is the material to be deposited, referred to as the target (in this case $\mathrm{ZnO}$ ) and the steel balls are ionised atoms, usually an inert heavy gas such as argon.

There are three predominant sputtering techniques: direct current (DC), radio frequency $(\mathrm{RF})$ and $\mathrm{RF}$ magnetron sputtering. The techniques differ by: deposition rate, depositing materials and complexity of the system.

\subsubsection{DC sputtering}

DC sputtering is the simplest of the techniques and can be used to deposit electrically conductive materials such as: nickel, silver, gold, titanium and zinc [76]. Figure 2.9 shows a simplified schematic of a DC sputtering chamber. 


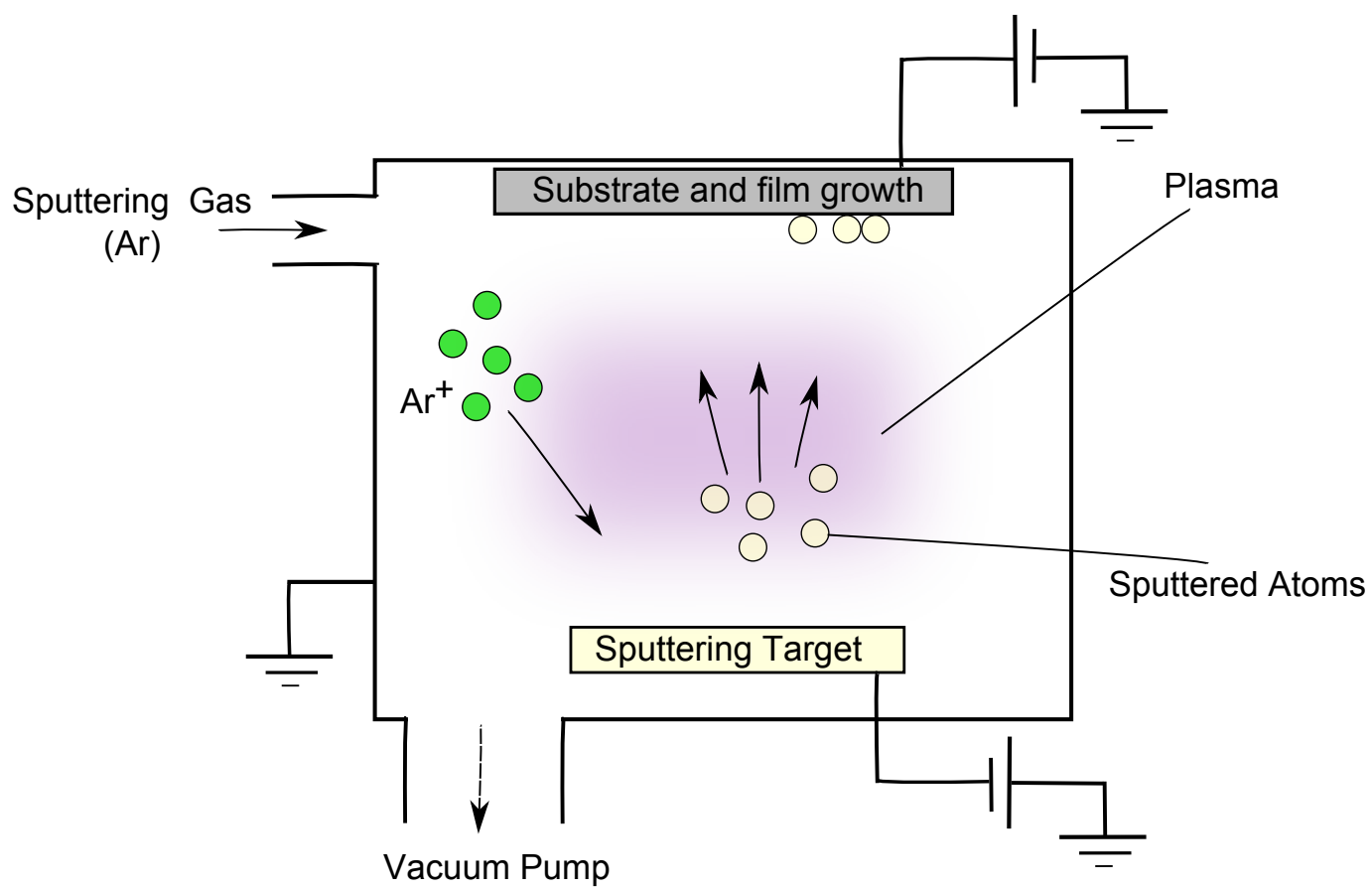

Figure 2.9: Simplified schematic of a DC sputtering system.

The chamber is evacuated down to a low pressure $\left(\approx \times 10^{-6}\right.$ mbar $)$ and then backfilled with an inert gas $\left(\approx \times 10^{-2}\right.$ mbar to $\approx \times 10^{-3}$ mbar $)$, argon (Ar) is most commonly used. An electric potential is applied between the substrate and target, inducing a positive polarity on the substrate and negative polarity on the target. The negatively charged target will repel free electrons inside the chamber. These electrons may collide with the surrounding neutral argon atoms. As they collide, electrons may be knocked off the argon gas leaving it as a positively charged ion $\left(A r^{+}\right)$. These positive ions are accelerated towards the target by the applied negative bias. When the ion collides with the target, one or more of the following processes may occur, as seen in Figure 2.10:

a) The positive ion maybe reflected off the target

b) The positive ion may cause surface damage to the target by forcing atoms into new lattice positions 
c) If the kinetic energy transferred is sufficient an atom will eject from the target material (sputtering).

d) A secondary electron may be ejected from the target

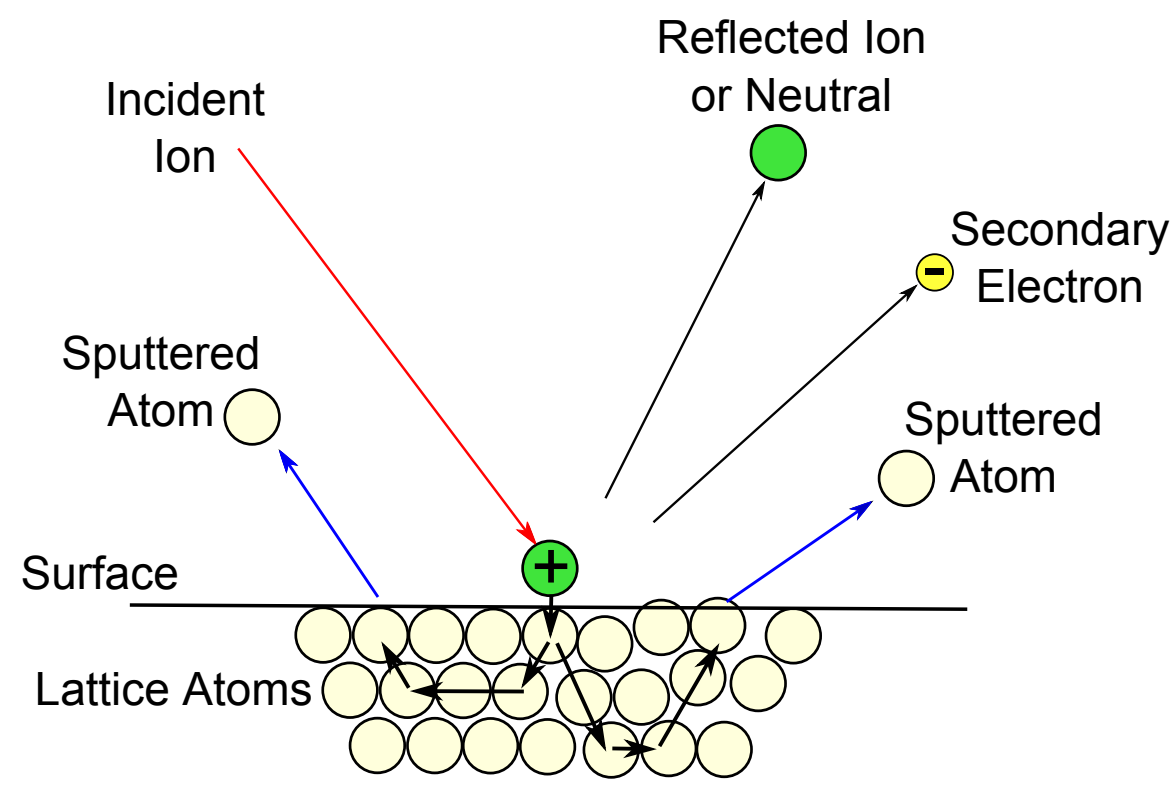

Figure 2.10: Interaction of ions at the target surface.

The process that takes place depends on the energy of the incident particle, the angle of incidence, the binding energy of the surface atoms and the mass of the colliding particles [76]. If a target atom is ejected, it travels across the chamber, condensing to form thin layers of the 'sputtered' material, on either the chamber walls or the substrate. When an ion collides with a free electron, due to energy conservation a phonon of light is emitted, creating the characteristic plasma 'glow'. The secondary electrons ejected from the target are used to sustain the plasma.

Since a negative potential is applied to the target, only electrically conductive materials can be deposited using this arrangement. The deposition of dielectric or insulating materials using this technique would lead to a build-up of charge on the target surface, requiring a very high voltage to sputter such materials [76]. An alternative technique is RF sputtering. 


\subsubsection{RF sputtering}

RF sputtering has a very similar arrangement to that of a DC system, except that for RF sputtering an alternating potential is applied between the target and substrate. This arrangement allows for the deposition of conducting, semiconducting and insulating materials, such as: $\mathrm{ZnO}$, aluminium oxide and silicon dioxide [76]. The basis for using a RF supply is that, effectively the number of electrons that arrive at the surface of the target while it is positive equals the number of cations arriving at the surface while it is negative. This overcomes the charge build-up on the surface of the target, as is the case with a DC supply. The frequency of the RF supply is dependent upon sustaining the plasma. The plasma is sustained by accelerating the electrons between the target and substrate such that they generate enough kinetic energy to collide with the sputtering gas creating ionised particles. Frequencies between 5 to $30 \mathrm{MHz}$ have been used, however $13.56 \mathrm{M} \mathrm{Hz}$ has become the standard because it has been reserved by the Federal Communications Commission for plasma processing [63].

\subsubsection{RF magnetron sputtering}

DC and RF sputtering are known to produce relatively low deposition rates. To overcome this, the rate can be increased by confining the electrons closer to the target, in an 'electron trap'. By constraining the plasma closer to the target, there is an increased probability that such an orbiting electron will strike an inert gas molecule causing it to become ionised. The electron trap is achieved by placing magnets either beside or underneath the target and producing a magnetic field at the target surface. When secondary electrons are emitted from the target, they are constrained by this magnetic field. These secondary electrons are effectively trapped in the region closest to the target, as shown in 2.11. By increasing the rate of ionised inert gas molecules the rate of ejected target material increases. 


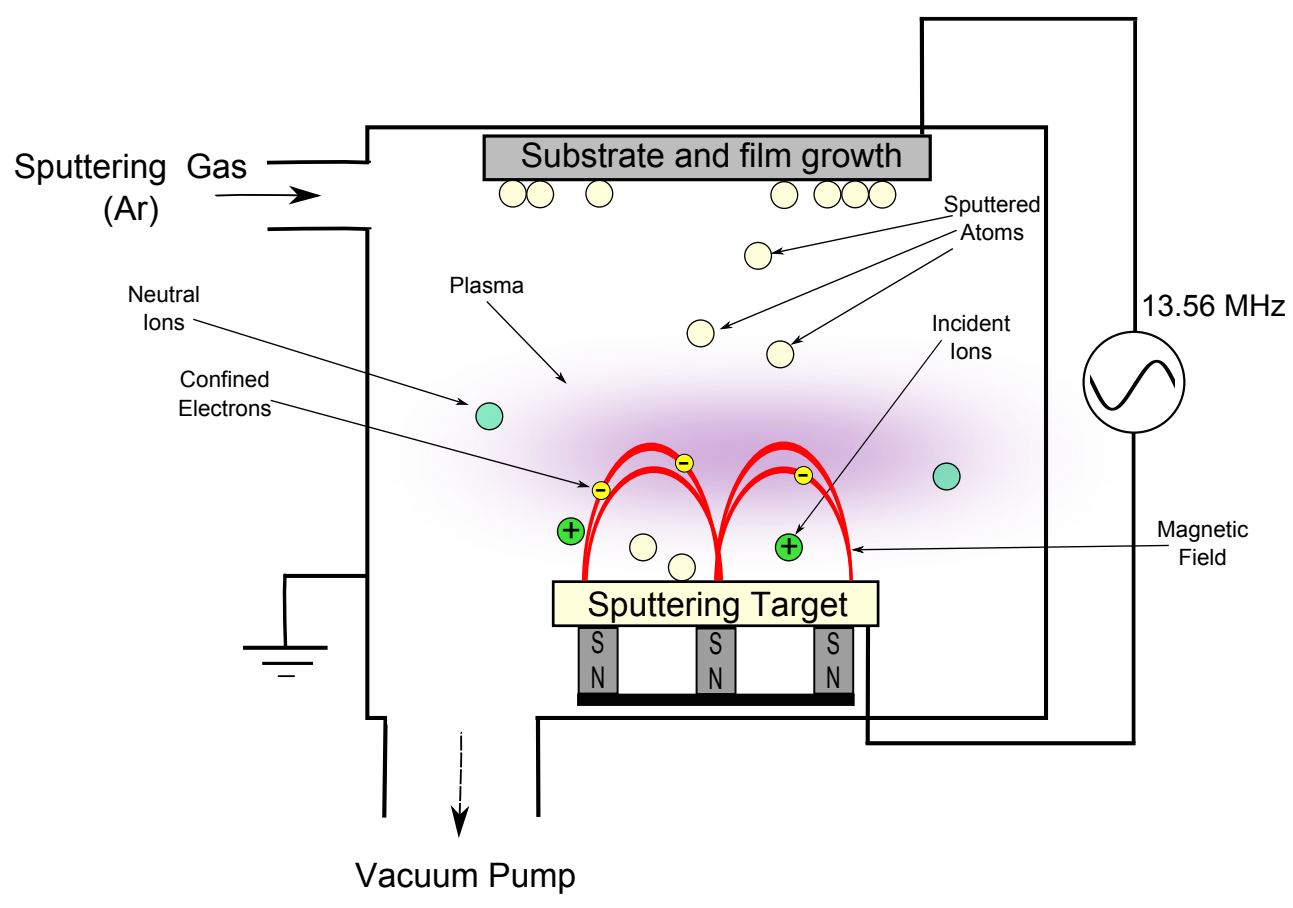

Figure 2.11: RF magnetron sputtering system.

Finally, it was mentioned earlier that sputtering allows the deposition of compound materials. There are two approaches to this. The first technique uses a target comprised of the compound material to be deposited for example using a $\mathrm{ZnO}$ target. The second is referred to as reactive sputtering, in this technique the deposited film is formed by a chemical reaction between the target material and a reactive gas. $\mathrm{O}_{2}$ is used to form oxides, $\mathrm{N}_{2}$ to form nitrides and $\mathrm{H}_{2} S$ to form sulphides. For example to form $\mathrm{ZnO}$, the deposition would involve using a pure zinc target in an oxygen enriched atmosphere. The composition of the film can be controlled by changing the ratio of the reactive gas to the inert gas. The technique used largely depends on the sputtering capability, such that deposition from a $\mathrm{ZnO}$ target is only possibly by RF sputtering.

For this project a RF magnetron sputtering system was used along with a $99.99 \%$ purity $\mathrm{ZnO}$ target. The advantage of using a $\mathrm{ZnO}$ target is that oxygen is released from the target surface during the deposition making 
it easier to achieve stable deposition conditions [30]. Whereas films deposited from a zinc target rely on the ratio of oxygen present during the deposition to grow $\mathrm{ZnO}$.

\subsubsection{Sputtering parameters for $\mathrm{ZnO}$}

The microstructure of a sputtered thin film is largely determined by the deposition conditions. One of the advantages of sputtering is the fine controllability over the deposition parameters [74]. Several important parameters can be altered during the deposition process. These are:

- RF power

- Chamber pressure

- Gas ratio (argon to oxygen ratio)

- Substrate temperature

- Substrate-to-target distance

- Substrate holder position (rotated or stationary)

By optimising these parameters films with desirable electrical, mechanical and optical properties can be obtained.

The use of sputtering $\mathrm{ZnO}$ for piezoelectric applications dates back to 1976 in the development of surface wave transducers [38]. Since then, sputtering of $\mathrm{ZnO}$ has been widely reported. Existing reports on sputtering $\mathrm{ZnO}$ can be found by $[29,78-84]$.

\subsubsection{Requirements of $\mathrm{ZnO}$ thin films for ultrasound trans- ducer applications}

There were several requirements the $\mathrm{ZnO}$ layers had to conform to in order to be useful as the piezoelectric element for ultrasound transducers. 
- Films should have the highest piezoelectric coefficient possible. Typically a value of $12 p C N^{-1}$ is seen as the bulk value [15]. Therefore, the piezoelectric coefficient should be as close to this value as possible.

- The first (but not the only) requirement, for a high piezoelectric response was for the films to be c-axis oriented, i.e. with the (0001) crystal direction perpendicular to the substrate surface. Typically $\mathrm{ZnO}$ films have a textured structure, and as such should consist of columnar (0001) grains.

- A second requirement for a high piezoelectric response is the film's surface should have the same polarity, i.e. either a zinc (positive) or oxygen (negative) top face, leading to a constructive piezoelectric response $[85,86]$.

- Films should have a high resistivity in order to allow the capacitorlike transducer structure to work as intended.

- Films should be of the correct thickness allowing operation at the desired frequency. This thickness can be estimated from the halfwavelength of the elastic wave. The generation of a $300 \mathrm{MHz}$ wave, requires an approximate film thickness of $10.7 \mu \mathrm{m}$. However, mass loading, from further fabrication of the transducer structure i.e. metal electrodes and substrate will influence this thickness-frequency relationship.

- To grow films of this magnitude, within a realistic time frame, high deposition rates needed to be maintained. The ideal deposition rates would then be $\geq 3 \mu \mathrm{m} /$ hour, although rates as low as $1 \mu \mathrm{m} / \mathrm{hour}$ should still be sufficient.

- The film needs a smooth surface morphology to facilitate further device fabrication e.g. the deposition of metal electrodes. 


\section{Chapter 3}

\section{Experimental Techniques}

This chapter will describe the experimental equipment used to deposit the $\mathrm{ZnO}$ films as well as explain the characterisation techniques used in analysing them.

\subsection{Sputter Deposition System: The Auto500}

The HHV Auto500 was used to deposit the $\mathrm{ZnO}$ films in this project [87]. This system was initially designed and sold by BOC Edwards (UK) as the Auto500 system. The vacuum chamber can be configured for either resistive evaporation, electron beam evaporation or sputter deposition. When configured for sputter deposition it can be equipped with up to three sputter sources and can use either DC or RF sputtering.

The HHV Auto500 as received by VUW was a very basic version of this system. It was equipped with a single sputter target and could only perform RF sputtering. A Dressler Cesar RF power supply capable of delivering $600 \mathrm{~W}$ and operating at $13.56 \mathrm{M} \mathrm{Hz}$ was used as the RF sputter supply. This was connected to an auto-matching network to balance the load. Ar gas is used as the standard sputtering gas, however reactive sputtering is made possible through a second gas line. In our case oxygen was used as the reactive gas when necessary. Figure 3.1 shows a block diagram of the 
complete sputtering system. The deposition chamber was evacuated by a rotary backing pump and a turbomolecular main pump. The power to the vacuum pumps as well as relevant valves and gauges were interfaced to and controlled by a programmable logic controller (PLC) via a touch screen control panel.

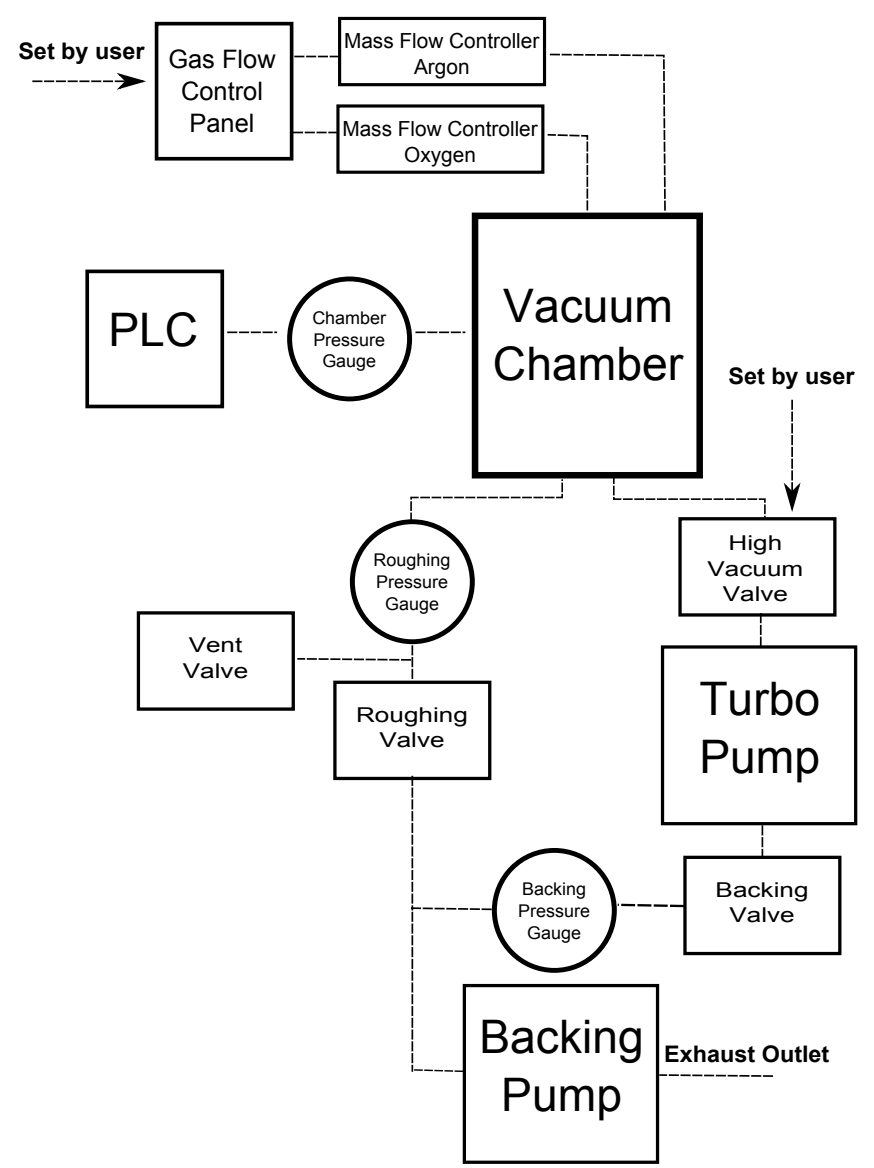

Figure 3.1: Block diagram of the sputter coater vacuum system.

Figure 3.2a shows a view of inside the sputter chamber while Figure $3.2 \mathrm{~b}$ shows the interfacing control panel. The single sputter source was offcentre of the chamber and could be closed off using a mechanical shutter. The substrates were mounted on a stainless steel substrate holder which was $\approx 260 \mathrm{~mm}$ in diameter and sat $\approx 150 \mathrm{~mm}$ above the target. The sub- 
strate holder could rotate about its axis by means of an external electric motor at a constant speed of $35 \mathrm{rpm}$.

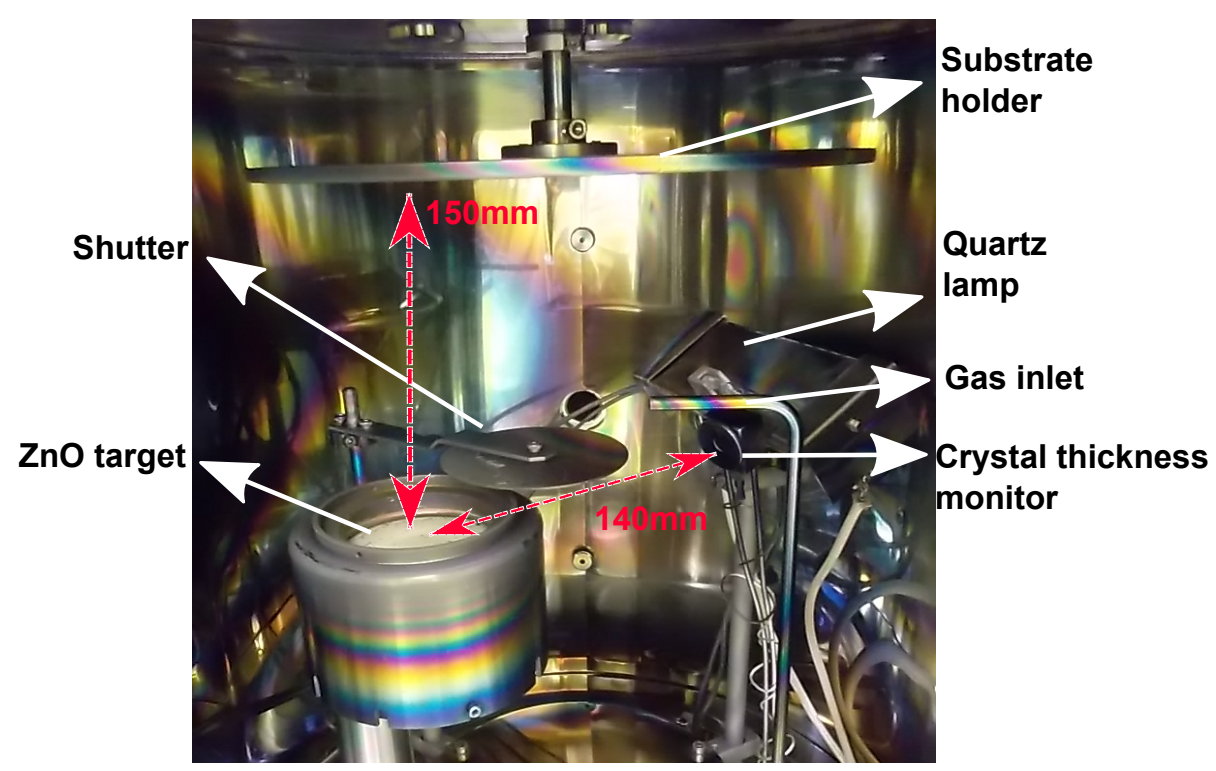

(a)

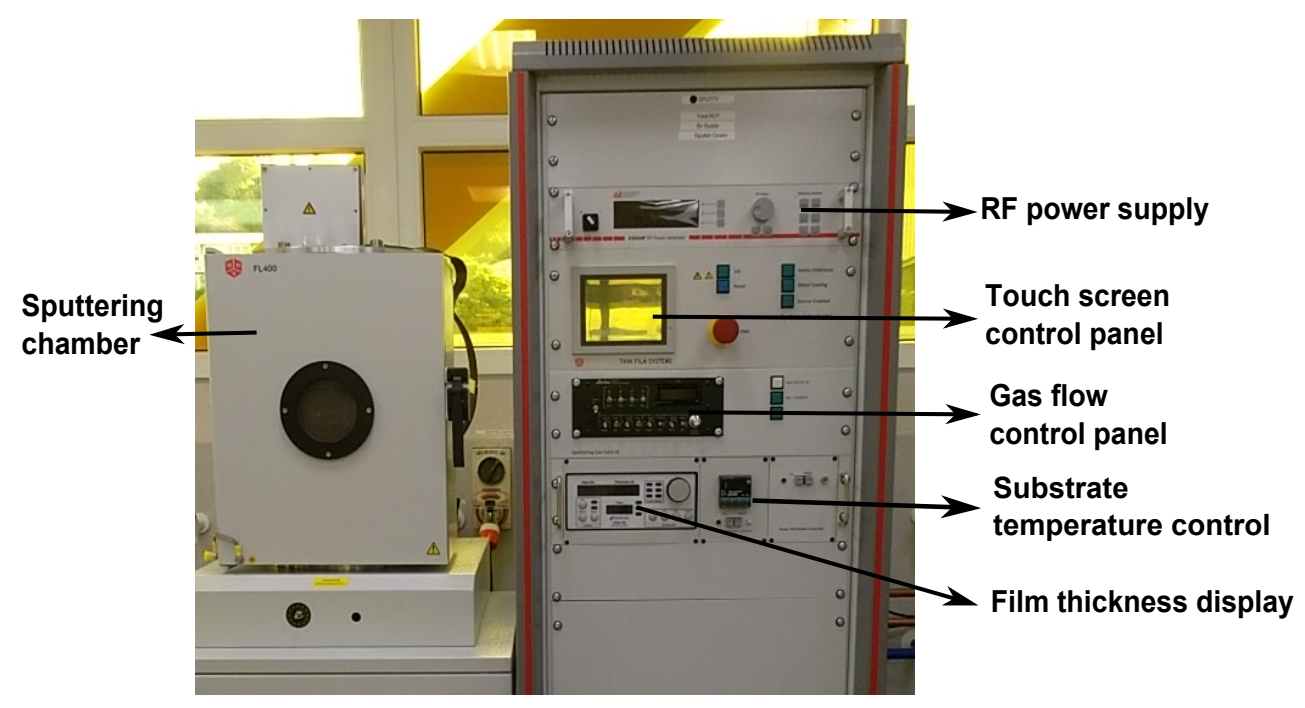

(b)

Figure 3.2: (a) Components inside the Auto500 sputter coater chamber, and (b) The complete sputtering system with the interfacing control panel on the right and deposition chamber on the left. 
A quartz crystal inside the chamber is used to monitor the thickness of the depositing layer, with the readout and control unit on the interfacing control panel. A quartz lamp inside the chamber, positioned underneath the substrate holder allows for substrate heating during the depositions. A thermocouple inside the chamber was used to monitor the substrate temperature with the readout and temperature control unit on the interfacing panel.

\subsection{Substrate Preparation}

It was anticipated that the $\mathrm{ZnO}$ films grown for ultrasound transducer applications would be deposited onto an amorphous substrate (aluminium oxide $\left.\left(\mathrm{Al}_{2} \mathrm{O}_{3}\right)\right)$. As such, to save cost during the initial development of the deposition procedure it was decided to perform our process development on cheap amorphous glass substrates. Initial depositions were performed on soda-lime glass (microscope slides). Later depositions also used borosilicate glass obtained from University Wafer [88] and Valley Design [89]. The slides were either used as received, or cut smaller by scribing with a diamond knife and snapped to size.

Before each deposition, the substrates underwent a cleaning process to remove contaminates from the surface and to improve bonding between the substrate and film. The following cleaning procedure was used:

(i) The substrates were placed into a beaker of high purity acetone and agitated in an ultrasonic bath for one minute, removing particles from the surface.

(ii) The substrates were removed and rinsed with high purity isopropanol (IPA) and dried with nitrogen gas.

(iii) If after close examination residue remained they were further cleaned with lint free wipes. 
In selected cases the substrates were then placed in a plasma cleaner for 1 minute using an $O_{2}$ plasma. No significant difference in the resultant film quality was observed from this step.

\subsubsection{Deposition Procedure}

$\mathrm{ZnO}$ films were deposited using the following procedure:

(i) Before each deposition the glass substrates were cleaned using the procedure specified in Section 3.2. The $\mathrm{ZnO}$ target was loaded into the magnetron target holder and shutter placed over, covering it. The substrate holder was removed from the chamber and substrates fixed to the holder using Kapton tape. The holder was loaded back into the chamber and fixed at the height of $150 \mathrm{~mm}$ above the target.

(ii) The chamber was evacuated and left for over an hour to reach pressures of $\approx 8 \times 10^{-6}$ to $8 \times 10^{-7}$ mbar.

(iii) The chamber was back-filled with $9 \mathrm{sccm}$ (standard cubic centimetre) of instrument grade argon, increasing the pressure to about $2 \times$ $10^{-2}$ mbar. If substrate heating was required, the quartz lamp was set to the desired temperature via the controller and switched on. If substrate rotation was required this was turned on as well.

(iv) The RF power supply was turned on and increased to $20 \mathrm{~W}$. If the plasma had not struck the shutter was quickly opened then closed, which usually resulted in the plasma striking. By opening the shutter the argon ions have direct access to the $\mathrm{ZnO}$ target, this increased the chance of the ions bombarding the target which would generated more secondary electrons to then collide with the surrounding argon atoms and generate the plasma.

(v) Once the plasma was struck, the RF power was increased to the desired deposition power. For this research, powers between 150 to 
$240 \mathrm{~W}$ were used. The argon gas flow was reduced to $6 \mathrm{sccm}$ decreasing the pressure to between $\approx 7 \times 10^{-3}$ to $1 \times 10^{-2}$ mbar, ready to deposit. If oxygen was required for a reactive deposition the oxygen flow was set and released into the chamber.

(vi) With the shutter closed, the system was left to pre-sputter for $\approx$ 5 mins to clean the target of containments such as dust or to remove any oxidation that may have occurred on the surface. After which the shutter was opened and sputtering was performed for the required time.

(vii) Once the deposition had finished, the shutter was closed. The RF power was decreased to $0 W$, the quartz lamp, substrate rotation and argon and oxygen gases turned off. The system was put into 'cycle' mode to evacuated down to $\approx 8 \times 10^{-4}$ mbar to pump away remaining gas.

(viii) The chamber was vented with nitrogen for $\approx 30$ seconds and the remainder done using atmospheric air. The initial nitrogen vent improves cleanliness of the chamber. The substrate holder was removed and samples taken off. The chamber was cleaned with a vacuum cleaner and wiped down with IPA.

\subsection{Thermal Annealing}

Selected samples were subjected to a post growth annealing procedure with the aim of relieving stress in the layers. Initially a crude annealing technique was used by placing samples on a hot-plate at $400^{\circ} \mathrm{C}$ for 1 hour. Results, as discussed in Section 4.3.4 showed that stress within the films had been released. As this proved to be a viable technique in improving the $\mathrm{ZnO}$ films a more sophisticated method was developed which allowed for higher annealing temperatures to be reached and more control of the 
surrounding atmospheric gas during the annealing process. Two annealing techniques were used:

(i) Inside the sputter coater chamber: While the samples were still fixed to the substrate holder and the chamber under vacuum, the substrate heater could be turned on to anneal the films at the highest obtainable temperature, which was found to be $250^{\circ} \mathrm{C}$ after modifications had been made to the sputter chamber geometry discussed in Sections 4.2 and 4.4. The pressure in the chamber was in the $\times 10^{-2}$ mbar range and with a flow of $1.9 \mathrm{sccm}$ of Ar and $0.1 \mathrm{sccm}$ of $\mathrm{O}_{2}$.

(ii) Inside a tube furnace: The samples were loaded into a ceramic boat and pushed into the middle of a quartz tube, the tube was placed into a furnace as shown in Figure 3.3. The samples were annealed at atmospheric pressure under a flow of $\mathrm{Ar}: \mathrm{O}_{2}$ of $95: 5 \%$. An automated program was used to ramp up the temperature, $20^{\circ} \mathrm{C} / \mathrm{min}$ until $200^{\circ} \mathrm{C}$ then $40^{\circ} \mathrm{C} / \mathrm{min}$ until the desired annealing temperature was reached, at which point the temperature would remain for the required annealing time. The furnace is capable of reaching a maximum temperature of $1200^{\circ} \mathrm{C}$, with temperatures of $400,600,750$ and $900^{\circ} \mathrm{C}$ used for this research. Figure 3.4 shows the temperature profile through the annealing furnace with an approximate position of where the ceramic boat was placed. The centre of the furnace is indicated at $0 \mathrm{~cm}$. It was assumed that the temperature profile is symmetrical about this centre point.

After the annealing process the samples were left to cool to $\approx 40^{\circ} \mathrm{C}$ before being removed, as thermal shock may lead to the films cracking. 


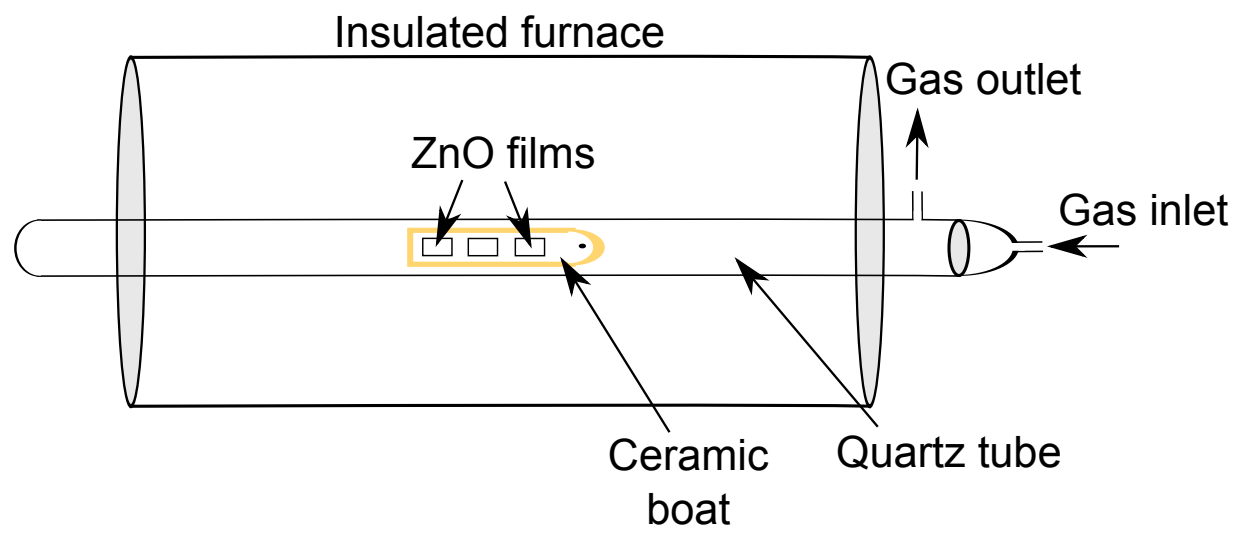

Figure 3.3: Annealing furnace housing the quartz tube.

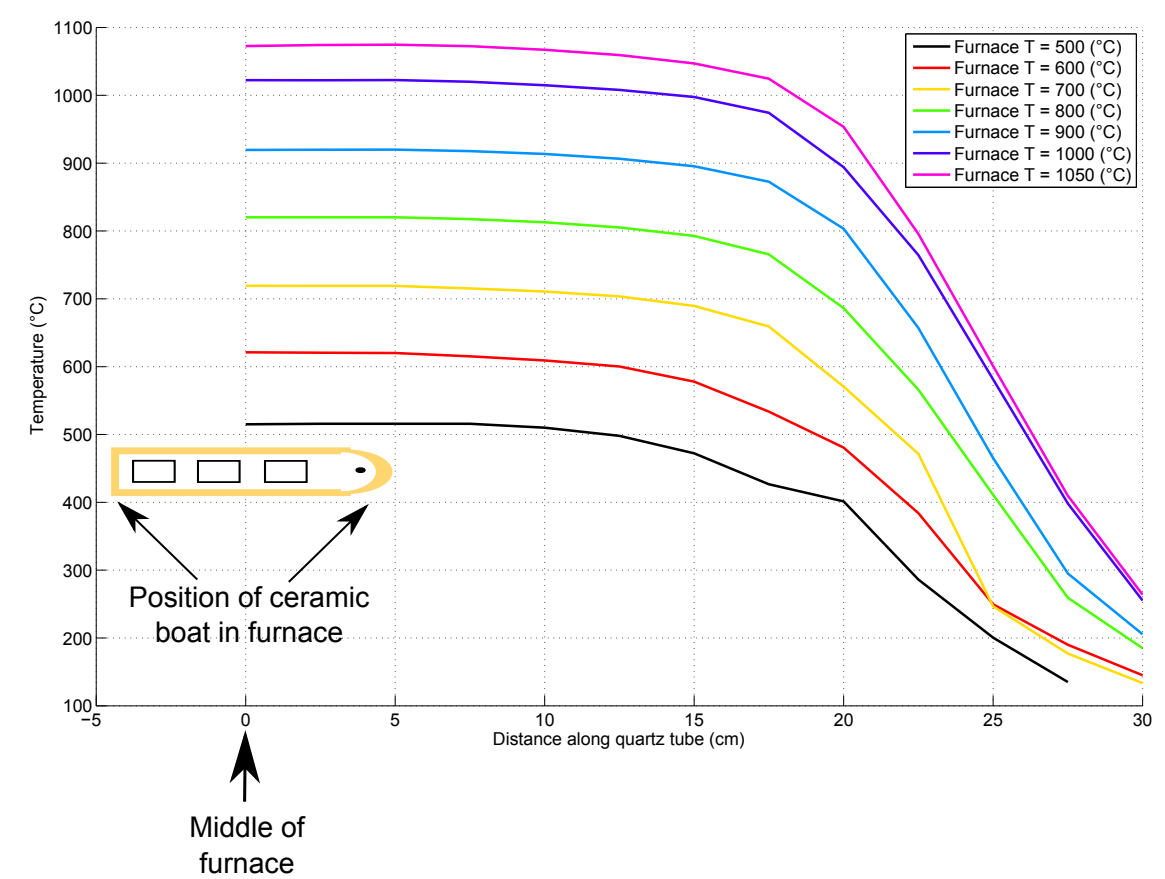

Figure 3.4: Temperature profile through the quartz tube inside the furnace showing approximate position of the ceramic boat inside the furnace. 


\subsection{Thin Film Characterisation Techniques}

After the deposition of a $\mathrm{ZnO}$ film, the films were analysed in terms of their thickness, crystallographic orientation, residual stresses and microstructure. This section will explain the characterisation techniques used in analysing the $\mathrm{ZnO}$ films.

\subsubsection{Dektak thickness profiler}

The Veeco Dektak 150 surface profiler (Dektak) was used to measure the film thickness by comparing the vertical height between two material interfaces. In this technique a sharp diamond stylus is placed in contact with the sample surface and dragged lightly across measuring the vertical profile of the sample, Figure 3.5.

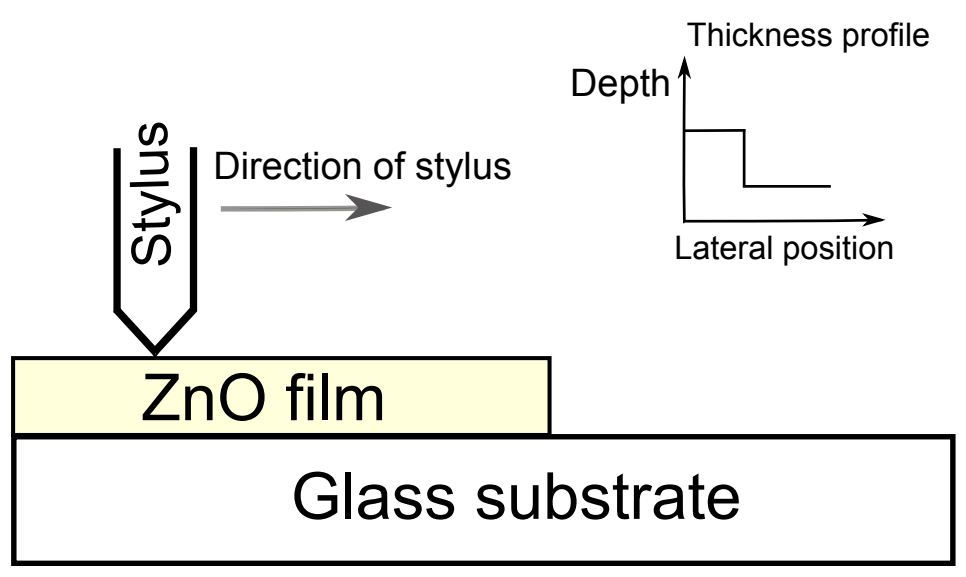

Figure 3.5: Schematic of Dektak surface profilometer used in measuring film thickness.

This technique can be used to explore the film's surface roughness, topography and step height. Thicknesses between $10 \mathrm{~nm}-524 \mu \mathrm{m}$ [90] could theoretically be measured, which was well within the range of our $\mathrm{ZnO}$ films. This technique allowed thickness measurements to be obtained within minutes, making it very efficient. However because the Dektak 
uses a surface contact technique, thicknesses could only be measured at the interface between two different vertical heights.

\subsubsection{UV-Visual Spectrophotometer:}

A UV-Visible spectrophotometer was used for optical transmission measurements through the sample over the range of 300 to $800 \mathrm{~nm}$. From this data a measurement of film thickness could be done as well as an estimate of the bandgap, a simple indication of a successful $\mathrm{ZnO}$ deposition. The transmission spectrum also provided an indication of the optical quality of the films. The UV-Visual spectrophotometer used was the Cary 100 Scan double beam, controlled by a computer running Varian's WinUV software. Figure 3.6 displays a simple schematic of the UV Spectrophotometer. It operates by generating light which is separated into different wavelengths using a monochromator. The single monochromatic light beam is then split into two parallel beams: One beam is used as the reference and passed through a referenced substrate and measured by a photo-detector. The other beam is passed through the sample and measured by a photo-detector. The measurement displayed is then the ratio of intensities between these two detected beams.

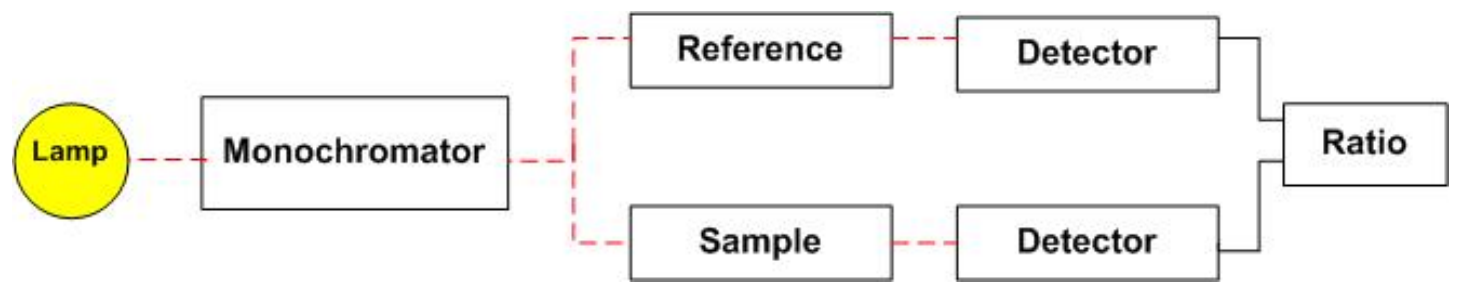

Figure 3.6: Simplified schematic of UV Spectrophotometer.

The interaction between the light beam at each material interface of the sample results in an interference pattern (or fringes), due to the partial reflection and transmission of the beam. These interactions could be used to estimate the thickness of the film. The output from the UV-Visual 
spectrophotometer was a spectrum of \% Transmission vs. wavelength, a typical example of which is shown in Figure 3.7.

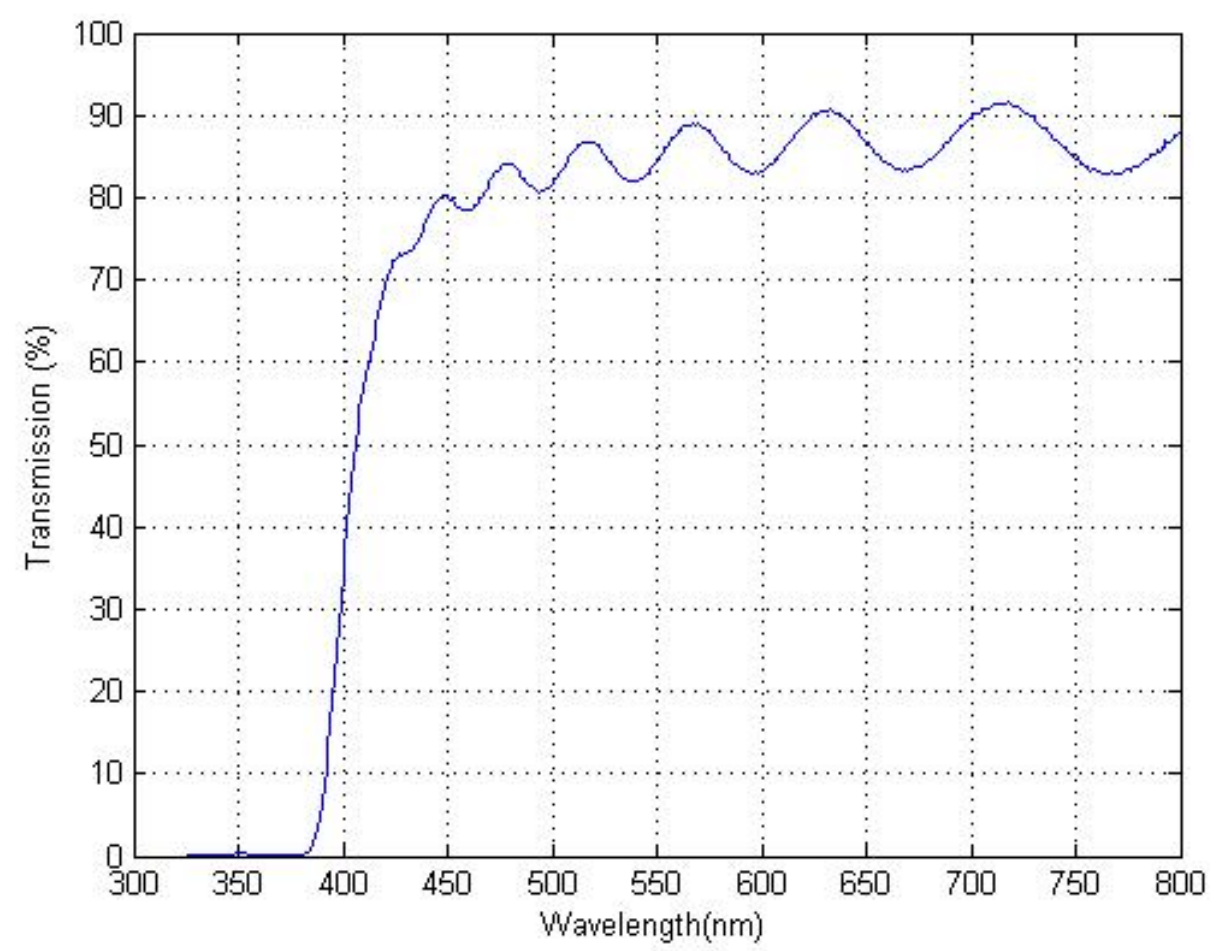

Figure 3.7: Transmittance spectra for a $\mathrm{ZnO}$ film.

From this spectrum information about the film's thickness and bandgap could be obtained through the following relationships:

The thickness of the film can be estimated by comparing the positions of either peaks or valleys from the transmission spectrum using the following equation [91],

$$
t=\frac{M \lambda_{1} \lambda_{2}}{2\left[n\left(\lambda_{1}\right) \lambda_{2}-n\left(\lambda_{2}\right) \lambda_{1}\right]}
$$

where $M$ is the number of oscillations between two peaks or two valleys, $\lambda_{1}, n\left(\lambda_{1}\right)$ and $\lambda_{2}, n\left(\lambda_{2}\right)$ are the corresponding wavelengths and refractive indices between two peaks or two valleys, respectively. 
The variation of refractive indices with wavelength for $\mathrm{ZnO}$ follows Cauchy's equation [92],

$$
n(\lambda)=A+\frac{B}{\lambda^{2}}+\frac{C}{\lambda^{4}}
$$

where $n(\lambda)$ is the refractive index, $\lambda$ is the wavelength, $\mathrm{A}, \mathrm{B}$ and $\mathrm{C}$ are coefficients for thin film $\mathrm{ZnO}$ are $A=1.9281, B=-1.1157 \times 10^{-5} \mu \mathrm{m}^{2}$ and $C=5.696 \times 10^{-3} \mu m^{4}$ [27].

As this technique involved measuring the interference pattern from the two $\mathrm{ZnO}$ surfaces, it was found that films $<300 \mathrm{~nm}$ did not provide sufficient fringes to perform thickness calculations. Therefore this technique was only used on films $>500 \mathrm{~nm}$.

The bandgap of a semiconductor material can be estimated from the edge in its optical transmission spectrum. Incident light with an energy less than the bandgap will not be able to excite an electron from the conduction band to the valence band and will be transmitted. Therefore the bandgap can be estimated by relating the absorption coefficient and the photon energy. The relationship between the absorption coefficient and transmission spectrum is described by the Beer-Lambert Law,

$$
\alpha=\frac{\ln \frac{1}{T}}{t}
$$

where $\alpha$ is the absorption coefficient, $T$ is the $\%$ transmission and $t$ is the thickness of the film. The bandgap can then be estimated by plotting the absorption coefficient with photon energy, a typical example of this is shown in Figure 3.8. 


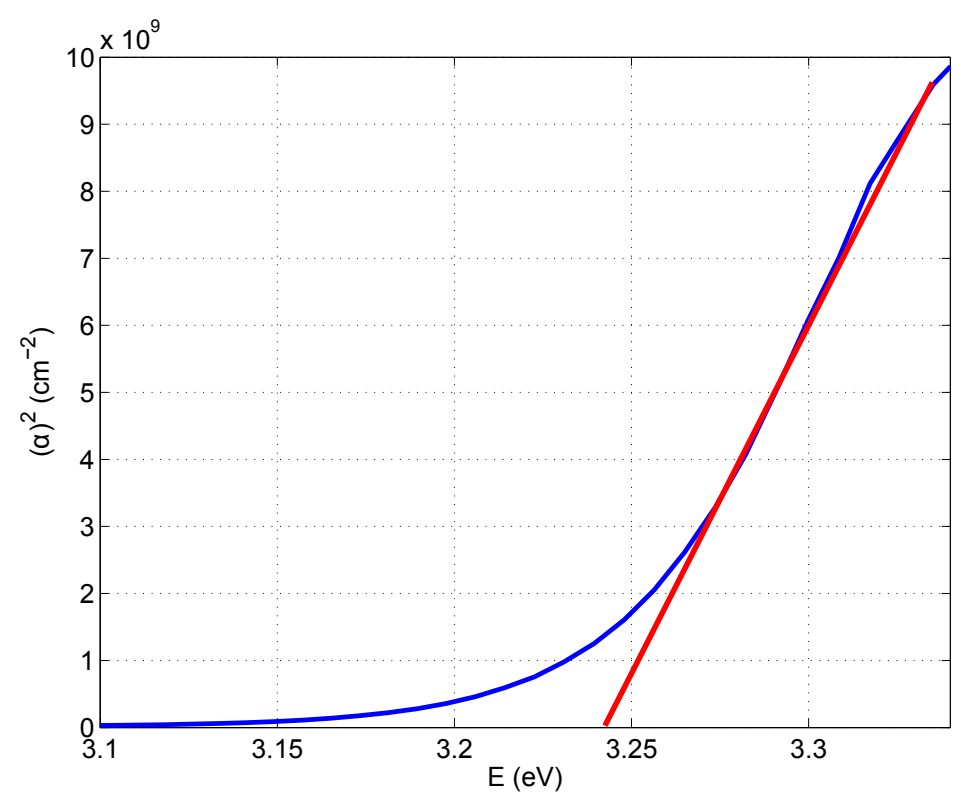

Figure 3.8: Absorption coefficient as a function of photon energy at thickness = $0.55 \mathrm{um}$. The bandgap is estimated to be $\approx 3.24 \mathrm{eV}$.

The photon energy is calculated using the Planck relation,

$$
E=\frac{h c}{\lambda}
$$

where $E$ is the photon energy, $h$ is Plancks constant, $c$ is the speed of light and $\lambda$ is the wavelength.

An estimation of the bandgap can be calculated by extrapolating the linear part of the $\alpha^{2}$ vs. E plot as shown in Figure 3.8 and finding where it cuts the $x$ axis (E) $[84,93,94]$.

\subsubsection{X-ray Diffraction (XRD) measurements}

$\mathrm{XRD}$ is a non-destructive technique used for determining crystal properties including structure, quality and chemical composition of bulk materials, thin films and powders. This technique involves the measurement of diffracted X-rays interacting with the crystal lattice of a sample which pro- 
vides information about the lattice parameters, present orientations and lattice strains. For this project the PANalytical X-ray diffraction system (XṔert PRO MPD) at VUW was used to investigate the crystalline properties of the $\mathrm{ZnO}$ films.

An X-ray beam is directed at the sample, a portion of the beam passing through a given lattice layer will collide with that layer while the remainder will continue on to the subsequent layers. In any lattice layer inelastic and elastic collisions are occurring between the beam and lattice atoms. If an inelastic collision occurs, energy is transferred between the beam and the atom, and the beam will reflect at an angle different to that of the incident, Figure 3.9a. If an elastic collision occurs, no energy is lost by the beam, but momentum is transferred and the scattered beam will reflect at the same angle as that of the incident, Figure 3.9b. The reflected X-rays from subsequent lattice layers interact with each other, resulting in constructive and destructive interference, producing a diffraction pattern. If the path travelled by these reflected beams is an integer multiple of the $X$-ray wavelength constructive interference occurs. In a periodic crystal lattice, peaks of constructive interference occur at particular incidence angles. This information is displayed on a graph as the reflected X-ray intensity vs. the reflected X-ray angle where the detector is positioned at the same angle from the point of incidence opposite the emitter.

Figure 3.10 graphically represents the possible reflections present from a standard $\mathrm{ZnO}$ reference powder, and Table 3.1 summarises these results [95]. 


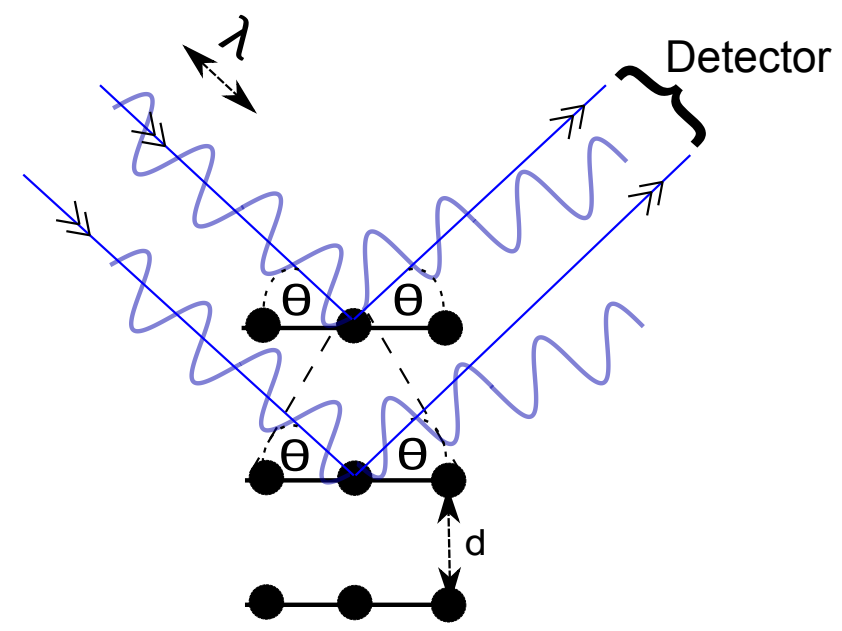

(a) Inelastic collision between the incident $\mathrm{X}$-ray and atom, $\mathrm{X}$-ray reflects at an angle different to that of incident.

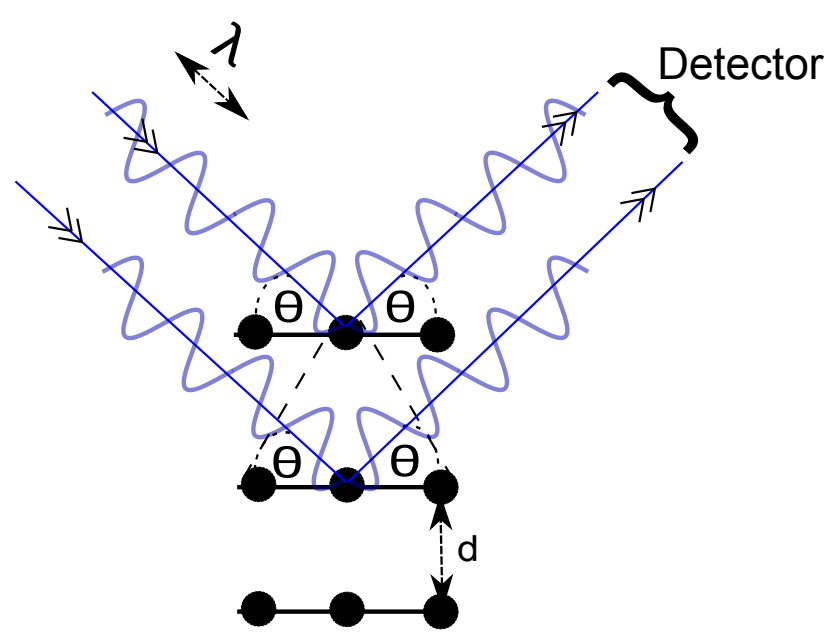

(b) Elastic collision between the incident $\mathrm{X}$-ray and atom, no energy transferred between $\mathrm{X}$-ray and atom therefore angle of incidences equals angle of reflection, resulting in constructive interference between the reflected X-rays

Figure 3.9: X-rays colliding with atoms in a crystal lattice. 


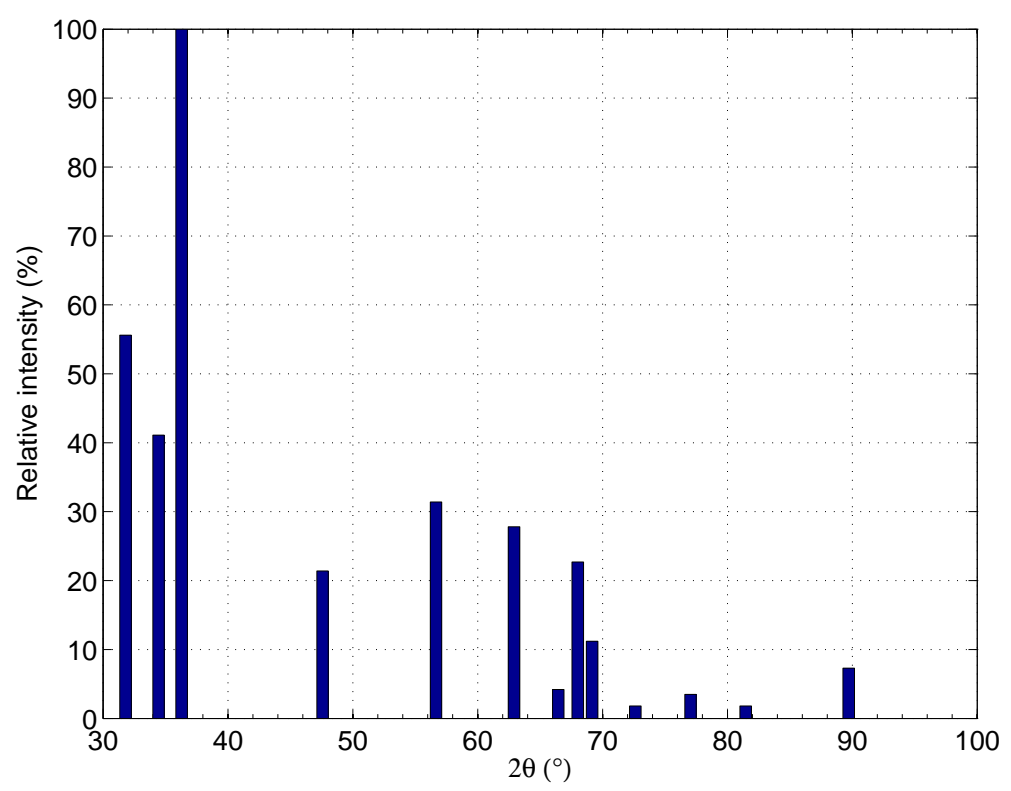

Figure 3.10: XRD spectra from powdered $\mathrm{ZnO}$ [95].

\begin{tabular}{|l|l|l|l|l|l|}
\hline $2 \theta\left({ }^{\circ}\right)$ & $d(\AA)$ & $h$ & $k$ & $l$ & $\begin{array}{c}\text { Relative } \\
\text { (intensity\%) }\end{array}$ \\
\hline 31.802 & 2.81160 & 1 & 0 & 0 & 55.6 \\
\hline 34.447 & 2.60150 & 0 & 0 & 2 & 41.1 \\
\hline 36.290 & 2.47350 & 1 & 0 & 1 & 100.0 \\
\hline 47.582 & 1.90950 & 1 & 0 & 2 & 21.4 \\
\hline 56.661 & 1.62320 & 1 & 1 & 0 & 31.1 \\
\hline 62.913 & 1.47610 & 1 & 0 & 3 & 27.8 \\
\hline 66.452 & 1.40580 & 2 & 0 & 0 & 4.2 \\
\hline 68.018 & 1.37720 & 1 & 1 & 2 & 22.7 \\
\hline 69.167 & 1.35710 & 2 & 0 & 1 & 11.2 \\
\hline 72.623 & 1.30080 & 0 & 0 & 4 & 1.8 \\
\hline 77.044 & 1.23680 & 2 & 0 & 2 & 3.5 \\
\hline 81.464 & 1.18050 & 1 & 0 & 4 & 1.8 \\
\hline 89.714 & 1.09210 & 2 & 0 & 3 & 7.3 \\
\hline
\end{tabular}

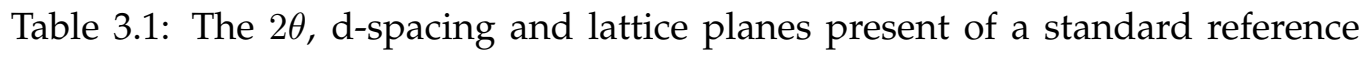
$\mathrm{ZnO}$ powder. Here $a=3.24982 \AA$ and $c=5.20661 \AA$ [95]. 
The peaks in the XRD spectra directly relate to the spacing of the atomic planes through Bragg's law,

$$
2 d_{h k l} \sin (\theta)=n \lambda
$$

where $d$ (referred to as the d-spacing) is the perpendicular spacing between the $h, k, l$ lattice planes in a crystal, $\theta$ is the reflected angle of the $\mathrm{X}$-ray (the peak position from the XRD spectrum), $n$ is an integer equivalent to the order of the diffraction peak, and $\lambda$ is the wavelength of the incident $\mathrm{X}$-ray.

For a hexagonal crystal structure such as $\mathrm{ZnO}$, the relationship between $d_{h k l}$ and the lattice parameters $a$ and $c$ plane is given by,

$$
\frac{1}{d^{2}}=\frac{4}{3}\left(\frac{h^{2}+h k+k^{2}}{a^{2}}\right)+\frac{l^{2}}{c^{2}}
$$

where $a$ and $c$ are the lengths of the slides of the primitive cell and $h, k, 1$ are the Miller indices.

The atomic planes reflecting the beam will further be determined by the structure factor, which for a hexagonal structure reflections are present if:

$h+2 k=3 n, l=$ even

$h+2 k=3 n \pm 1, l=$ even

$h+2 k=3 n \pm 1, l=$ odd

All the reflections are absent if $l=$ odd and $h+2 k=3 n$ [96].

The XRD spectra also provides information about the crystal quality such as crystal defects or any factors that will lead to a spread in the $d_{h k l}$ values for a diffraction plane which will produce a broadening of the $h k l$ peak. The full width half maximum (FWHM) value of a diffraction peak is then often quoted as an indication of crystalline perfection in a material.

$\mathrm{ZnO}$ demonstrates a piezoelectric response along its c-axis, which is the (002) plane. For bulk $\mathrm{ZnO}$ the (002) plane occurs at a $2 \theta$ value of $34.45^{\circ}$ [95]. Therefore, ideally we want our XRD spectra to show a narrow, high 
intensity peak occurring at $\approx 34^{\circ}$ with no other peaks present (except the reflection at $\approx 72^{\circ}$ ). This will indicate that the $\mathrm{ZnO}$ films have a c-axis orientation and therefore should show a strong piezoelectric response.

The XRD data can be used to calculate the stress within a film. If the deformation of the film is uniform over a relatively large distance this strain will cause a change in the lattice spacing compared to that of the stress free value. This change in lattice spacing can be seen as a shift in the $2 \theta$ peak position of an XRD spectrum. Figure 3.11a demonstrates a stress-free hexagonal lattice structure and its corresponding $2 \theta$ peak position. In Figure $3.11 \mathrm{~b}$, the hexagonal lattice is subjected to compressive stress resulting in the lattice shrinking in the horizontal direction and to satisfy Poisson's ratio expanding in the vertical. This can be seen as a decrease in the $2 \theta$ peak position. The opposite is also true if the film expands, resulting in a increase in the $2 \theta$ peak position, this is referred to as tensile stress.
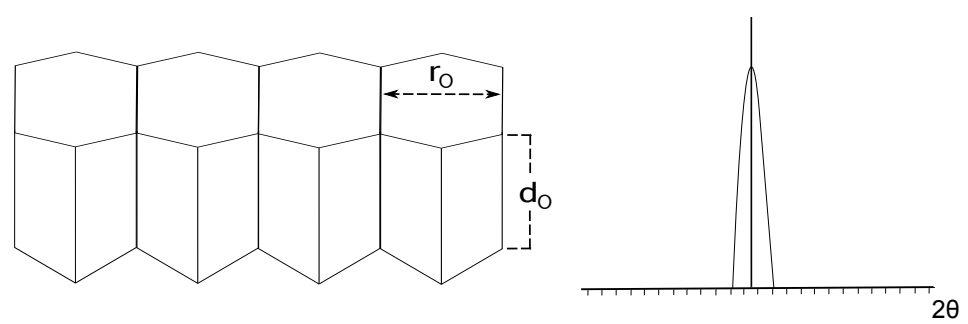

(a) Unstressed lattice

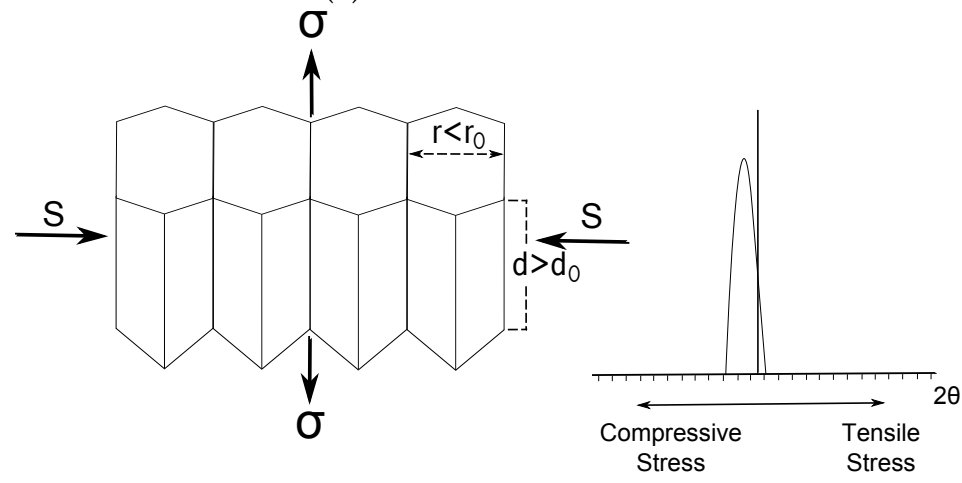

(b) Lattice subjected to stress

Figure 3.11: Demonstrating a shift in the $2 \theta$ peak position relative to a stress and strain seen by the lattice. 
The residual stress within a film may be calculated using Hooke's law. A solid experiencing a stress $S$, undergoes a proportional strain $\sigma$, related by its elastic compliance $s$,

$$
S=s \sigma
$$

The elastic compliance can be estimated using the elastic constants along the different orientations of $\mathrm{ZnO}$, and for a hexagonal structure is related by [97],

$$
s=\frac{2 c_{13}^{2}-c_{33}\left(c_{11}+c_{12}\right)}{2 c_{13}} S=-232.81 G P a
$$

where the elastic stiffness constants are given in Table 3.2

\begin{tabular}{|l|l|}
\hline $\begin{array}{c}\text { Elastic Constant } \\
\text { Orientation }\end{array}$ & $\begin{array}{l}\text { Value } \\
(\mathrm{GPa})\end{array}$ \\
\hline$c_{11}$ & 208.8 \\
\hline$c_{12}$ & 119.7 \\
\hline$c_{13}$ & 104.2 \\
\hline$c_{33}$ & 213.8 \\
\hline
\end{tabular}

Table 3.2: Elastic constants of $\mathrm{ZnO}$ as measured by $[97,98]$.

The strain $\sigma$ along the c-axis, is a measure of how much the films lattice parameter diverges from the bulk value and can be evaluated by [99],

$$
\sigma=\frac{\left(c-c_{o}\right)}{c_{o}} \times 100 \%
$$

where $c_{0}$ is the unstrained lattice parameter of $\mathrm{ZnO}(5.2066 \AA$ refer to Table 3.1) and $c$ is the lattice parameter of the strained $\mathrm{ZnO}$ calculated by Equation 3.6.

Using equations 3.7, 3.8 and 3.9 the residual stress in the thin film can be shown to be, 


$$
S=\frac{2 c_{13}^{2}-c_{33}\left(c_{11}+c_{12}\right)}{2 c_{13}^{2}} \times \frac{\left(c-c_{o}\right)}{c_{o}} S=-232.81 \times \frac{\left(c-c_{o}\right)}{c_{o}}
$$

\subsubsection{Scanning Electron Microscopy (SEM)}

An SEM was used to investigate the surface morphology of the $\mathrm{ZnO}$ films. SEM is considered a non-destructive technique used to generate high resolution images of the surface of objects, providing significant characterisation of solid materials. These high resolution images are produced using a highly focused scanning electron beam. The signal generated from the electron-sample interactions contains information about the sample's surface topology, chemical composition, elemental analysis and crystallographic structure of the sample.

An electron gun is used to thermionically emit a primary electron beam, with an energy ranging from $0.2 \mathrm{keV}$ to $40 \mathrm{keV}$ depending on the conductivity of the sample. The beam is focused through one or two condenser lenses and deflected through a pair of scanning coils or deflector plates to allow the beam to scan in the $x$ and $y$ directions over the surface of the sample. Where the primary beam makes contact with the sample, there is an energy exchange between the electron beam and sample surface resulting in the generation of secondary electrons (used to create SEM images), backscattered electrons (BSE, used for analysis of contrasts of chemical composition), diffracted backscattered electrons (EBSD, these signal are used to determine crystal structure) and photons (used for elemental analysis).

The Jeol JSM 6500F was the scanning electron microscope used to image the $\mathrm{ZnO}$ films. It had the capability to produce high resolution images down to the nano scale $(\approx 5 \mathrm{~nm})$ level, chemical composition and spot elemental analysis. For this project only imaging and chemical composition was used, providing visual information about the film's grain size, shape and consistency. Sample preparation included depositing a thin layer of 
carbon onto the samples as a conduction layer. This was to avoid charge build up on the surface of the sample, which makes imaging harder. The samples were also placed into a desiccator overnight to extract moisture from the sample, in order to assist the SEM in reaching the desired vacuum.

The SEM images obtained during this study were of the film's top surface and cross section. Top surface images were used to analyse the film's grain sizes, shape and consistency. Cross sectional images provided information about the thickness and columnar structure of the film. 


\section{Chapter 4}

\section{Results}

In this chapter, the experimental results from this thesis are presented. As was explained in Chapter 1, the focus of this project is to develop a fabrication technique for piezoelectric thin films for use in ultrasonic transducers. Specifically c-axis oriented $\mathrm{ZnO}$ films deposited by RF magnetron sputtering at moderately high deposition rates were required. The chapter begins by evaluating the HHV Auto500 sputtering system as it was received from the manufacturer. The as-received sputter chamber geometry was found to produce low deposition rates, and as a result modifications were made to the chamber geometry in an effort to increase the deposition rate. The influence of various deposition parameters on film microstructure was then investigated and a two-step deposition process was introduced to produce the desired film orientation at sufficiently high deposition rates. At this stage of the project it was also found that some of the films began to crack and peel away from the substrate. Stress within the film was determined to be the likely cause and a solution proposed to reduce stress during the deposition process. To help decrease the stress within the deposited $\mathrm{ZnO}$ films, post growth annealing was also investigated. A further modification was then made to the sputter system with the introduction of a new substrate holder. This allowed both, the deposition of films with better uniformity as well as in situ annealing of the films. The results are 
then concluded with a proposed four-step deposition growth procedure that should produce films with an optimised microstructure.

\subsection{Initial sputter system evaluation}

This work represents the first use of a new sputter deposition system in a new clean room facility at VUW. In the initial part of this work, the capability of this received sputter system, for the production of $\mathrm{ZnO}$ films was evaluated in general, but in particular it was aimed to establish if any modifications would be required for depositing $\mathrm{ZnO}$ films to our specific specifications (see Section 2.4.3).

Two types of experiments were performed during this equipment evaluation phase of the project:

(i) Simulated 'dry-run' experiments, in which no substrate was loaded but the sputter characteristics and performance were measured using the systems built-in instrumentation. For example, the operating pressure as a function of Ar gas flow and baffle valve setting was measured in this way. The deposition rate, as measured from the thickness monitor, could also be used to determine the dependence of the deposition rate on factors such as gas flow and RF power. The advantage of performing dry-run experiments to test these system parameters was that we were able to perform a large number of experiments in a relatively short time and saved on substrates and target material.

(ii) Once the initial deposition parameters had been evaluated in dryrun experiments, conventional experiments commenced. The deposition and measurement procedures used are outlined in Chapter 3. Films were deposited at various RF power, Ar flow, substrate temperatures and using substrate rotation, and the influence of these 
parameters on film quality was evaluated. All films were grown on $75 \times 25 \mathrm{~mm}$ soda lime glass substrates and at a fixed target-tosubstrate distance of $150 \mathrm{~mm}$, as determined by the sputter chamber configuration. During most depositions a typical deposition time of 60 minutes was employed, although in selected cases deposition times as short as 20 minutes were used.

\subsubsection{Setting of chamber pressure}

Chamber pressure was the first parameter investigated in our study. Setting of the chamber pressure could be done by mechanically setting the position of the high vacuum valve during 'throttle' pumping. A mechanical adjustment will determine the position of the high vacuum valve, and the resultant at equilibrium pressure is a balance between the pump speed and the flow of gas ( $\mathrm{Ar}$ and $/$ or $\mathrm{O}_{2}$ ) into the chamber. Any change in $\mathrm{Ar} / \mathrm{O}_{2}$ flow rate will thus change the chamber pressure for a fixed setting of the valve. It is also expected that other factors such as substrate temperature or RF power has the potential to influence the chamber pressure.

The high vacuum valve was then manually set to a selected position and the Ar gas with a flow rate between $1 \mathrm{sccm}$ and $10 \mathrm{sccm}$ allowed to flow into the chamber. For each Ar flow the chamber pressure was observed. The valve was then adjusted to the next position and the procedure repeated.

The observed chamber pressure is plotted against Ar flow for three high vacuum valve settings in Figure 4.1. For comparison, the equivalent curve supplied by the HHV Auto500 manual is also shown. In this Figure the positions 1 through 3 correspond to an increase in valve opening and hence an increase in chamber pressure. 


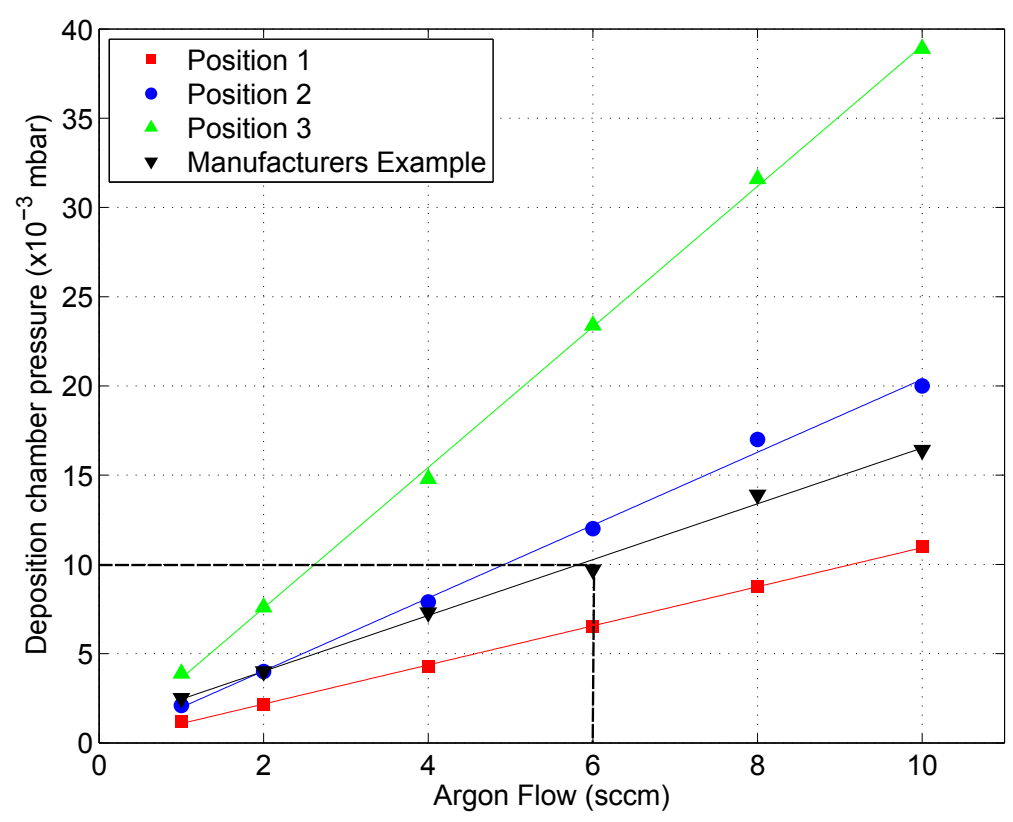

Figure 4.1: Deposition chamber pressure as a function of Ar gas flow as measured by our system and as stated in the HHV Auto500 manufacturer's manual. The black dotted line indicates the typical Ar flow, and associated working pressure that was used during most of our initial depositions.

All our subsequent deposition experiments were performed with a fixed gas flow rate. This meant that the position of the vacuum valve had to be manually adjusted in order to bring the deposition pressure within the desired range. As a guide we tried to adjust the gas flow-pressure relationship as close as possible to the manufacturer's example (black triangles in Figure 4.1). However, this was complicated by the fact that no position sensing or feedback of the valve position was incorporated, so the procedure was not always reliable and day-to-day drift was often observed. Typical gas flow rates of $6 \mathrm{sccm}$ of Ar were used during depositions, and the high vacuum valve was then adjusted to provide a chamber pressure of $\approx 1 \times 10^{-2}$ mbar, as indicated by the black dotted line in Figure 4.1. 


\subsubsection{Influence of RF power on deposition rate}

The influence of RF power on the deposition rate of $\mathrm{ZnO}$ was investigated in a series of dry-runs, where no substrates were used, but the deposition rate, simply measured by the built in thickness monitor and used as an indication of process conditions. Although the deposition rate observed from the thickness monitor will be different from the actual deposition rate on the substrate, it can be expected that the rates will be related by a geometrical factor (the tooling factor). The use of the thickness monitor will thus provide a first indication of the dependence of the process on the deposition parameters.

The RF power was set to values between $180 \mathrm{~W}$ and $320 \mathrm{~W}$ and the deposition rate, averaged over a period of 10 minutes, was measured on the thickness monitor. The results in Figure 4.2 show that observed deposition rate increased linearly from $\approx 2 \mathrm{~nm} / \mathrm{min}$ at $180 \mathrm{~W}$ to $\approx 5 \mathrm{~nm} / \mathrm{min}$ at $320 \mathrm{~W}$ over this range. This increase in deposition rate with RF power was expected, as increasing RF power increases the proportion of Ar ions in the plasma. With more ions bombarding the $\mathrm{ZnO}$ target, more of the target material is sputtered. This linear relationship between the RF power and deposition rate is widely documented [100-102]. 


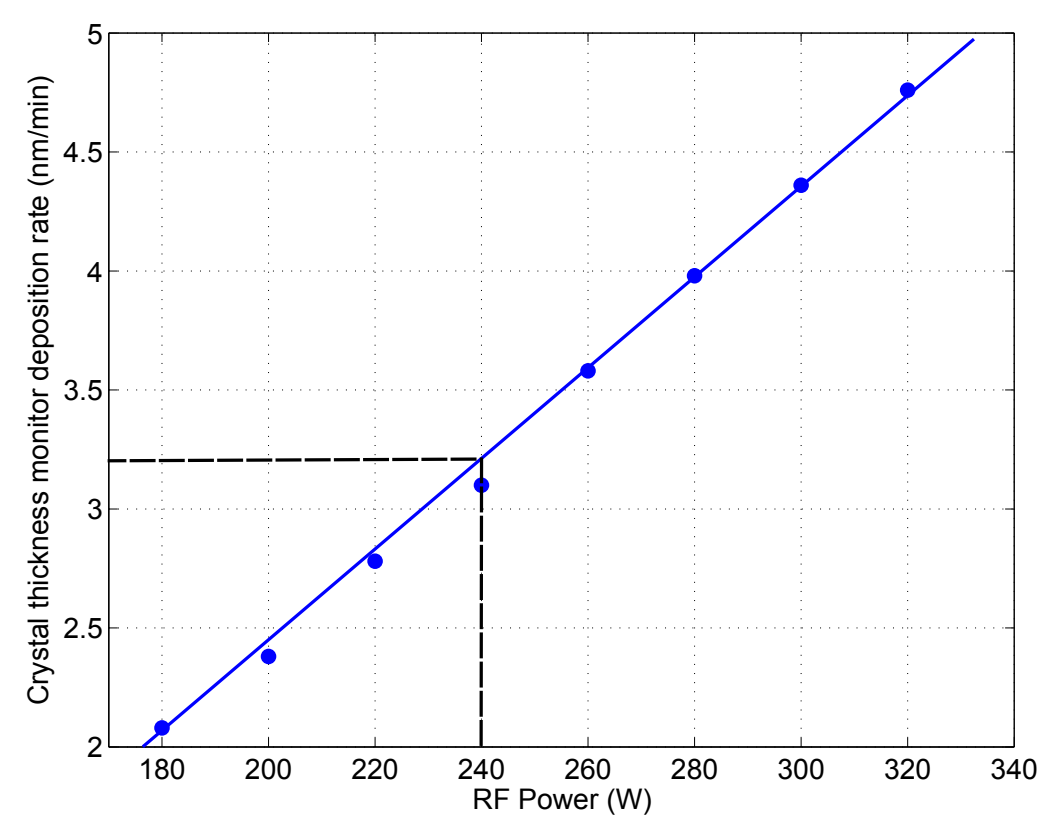

Figure 4.2: Variation in deposition rate as a function of the RF power, film thickness was determined by the thickness monitor inside the chamber. The black dotted line indicates the typical RF power used in later depositions.

\subsubsection{Influence of Ar flow on deposition rate}

The influence of Ar flow on the observed deposition rate was similarly measured in a series of dry-runs with deposition rates measured by the thickness monitor. The Ar flow was varied between $1 \mathrm{sccm}$ and $15 \mathrm{sccm}$ for different RF powers. It should be noted that across this series of experiments chamber pressure was not kept constant, varying from $3 \times 10^{-3}$ mbar at $1 \mathrm{sccm}$ of Ar to $2.3 \times 10^{-2}$ mbar at $15 \mathrm{sccm}$ of Ar. The results from these experiments are shown in Figure 4.3. It is seen that for each RF power there is a similar trend between Ar gas flow rate and deposition rate. As the Ar flow is increased, an increase in the deposition rate is initially observed. At a flow of $\approx 5 \mathrm{sccm}$ a maximum in the deposition rate is observed, above which the deposition rate decreases. At higher Ar flows there are more gas atoms present in the chamber, this results in an increase in collisions 
between the sputter $\mathrm{Zn}$ and $\mathrm{O}$ atoms with the surrounding particles reducing the chance of the atoms reaching the substrate surface and effectively slowing down the deposition rate. These curves indicate 'optimal' deposition rates occurring between $\approx 4-6 \mathrm{sccm}$. As expected, the maximum deposition rate is obtained with the highest RF power used $(360 \mathrm{~W})$ but it is interestingly to note that the deposition rate also appears to drop off the quickest with increasing flow. The rate at which the deposition rate drops off past the optimal range is also a function of RF power, with the greatest decrease observed for high RF powers.

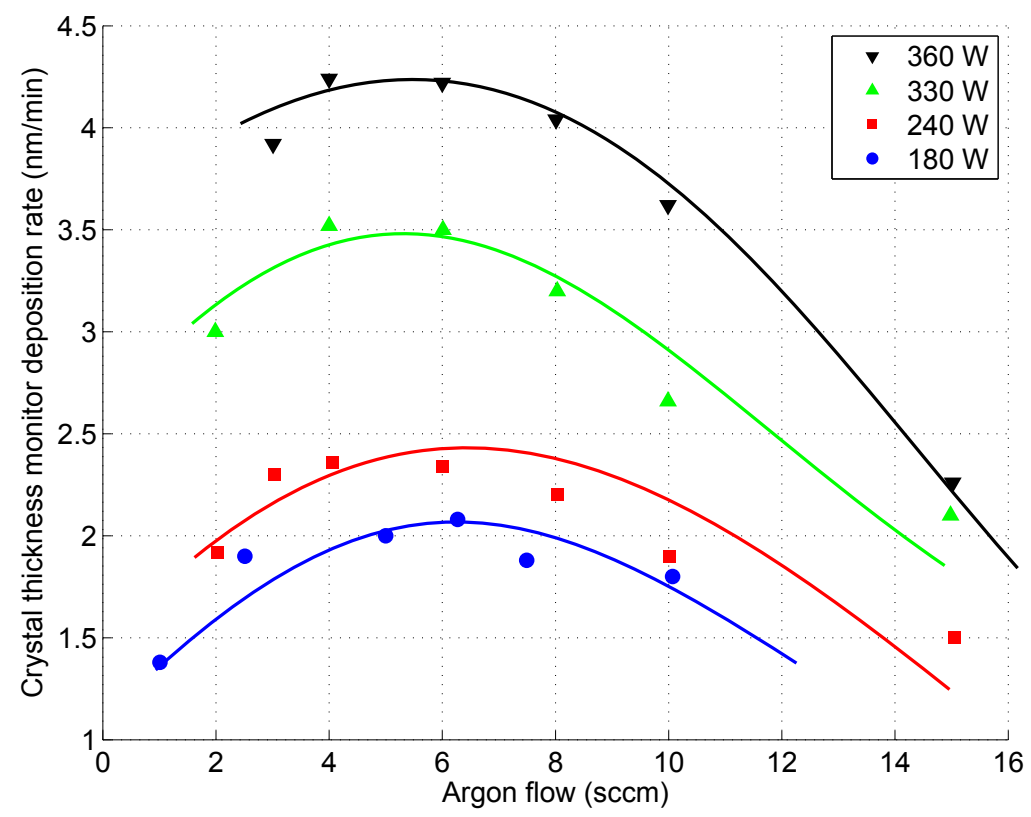

Figure 4.3: Variation in deposition rate as a function of Argon flow over a range of RF powers. All other parameters held constant: RF power $=180-360 \mathrm{~W}$, chamber pressure $=3 \times 10^{-3}$ mbar to $2.3 \times 10^{-2}$ mbar .

The results from Figure 4.3 suggest that maximum deposition rates can be achieved when depositing with Ar flows of $\approx 4-6 \mathrm{sccm}$. During the course of this work an Ar flow of $6 \mathrm{sccm}$ was primarily used in depositions. 


\subsubsection{Control of substrate temperature}

The system was provided with a quartz lamp inside the deposition chamber that could be switched on and its power controlled to provide substrate heating during depositions. The range of substrate temperatures that could be achieved were evaluated and it was found that a maximum of $\approx 100^{\circ} \mathrm{C}$ could be reached with the quartz lamp heating. This poor heating was in part due to the distance between the lamp and the substrate holder (see Figure 3.2a). Even without the lamp, the substrate can be expected to heat up during a deposition, as the substrate will be bombarded with energetic particles from the plasma.

The effective substrate temperature was measured for different RF powers and the results are shown in Figure 4.4. The temperatures recorded here represent the final temperature reached (after $\approx 20$ minutes) at the specified RF power. This would indicate a rise of $\approx 1^{\circ} \mathrm{C}$ for every increased $20 \mathrm{~W}$ across the range of $200 \mathrm{~W}$ to $320 \mathrm{~W}$.

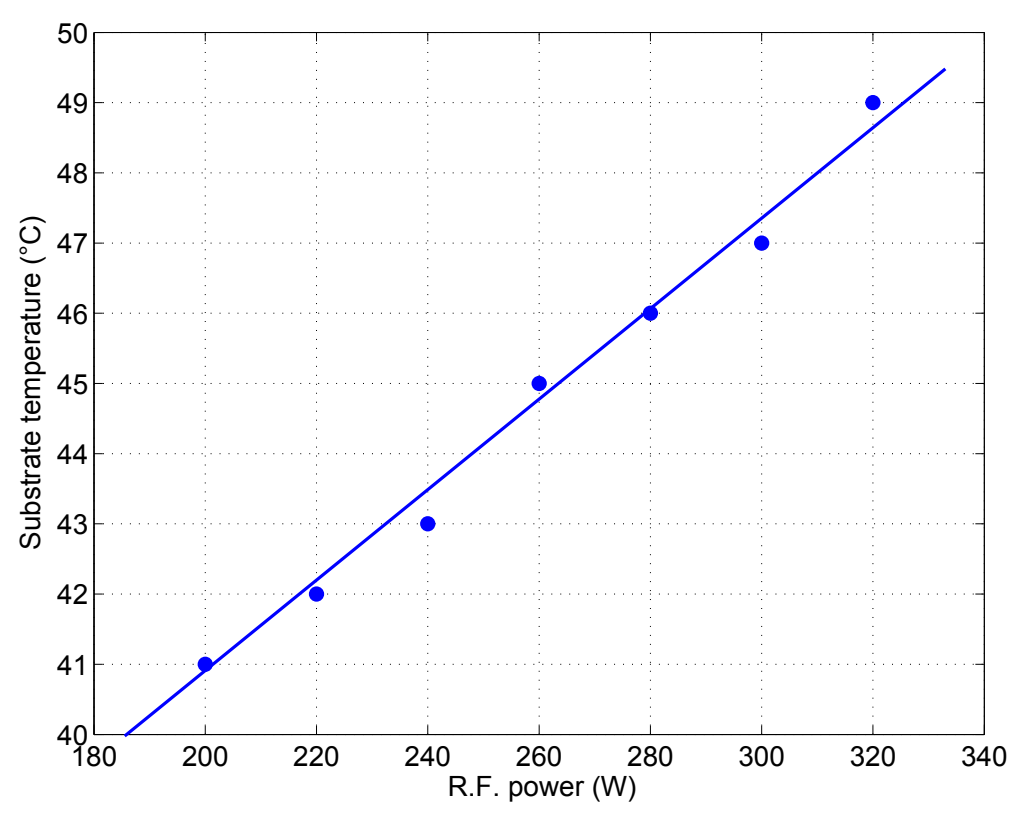

Figure 4.4: Substrate temperature increases linearly with increasing RF power over the range of $200 \mathrm{~W}$ to $320 \mathrm{~W}$. 


\subsubsection{Influence of deposition parameters on film properties}

The results from the initial series of dry-run experiments were undertaken to select initial deposition parameters for $\mathrm{ZnO}$ depositions. Further experiments now consisted of conventional depositions onto glass substrates and the evaluation of these films by the procedures described in Chapter 3 in order to optimise the deposition process. However, initial characterisations of the resultant films were performed primarily to confirm that our films actually were $\mathrm{ZnO}$. The results of these early evaluations are presented below.

The first proof that the films were indeed $\mathrm{ZnO}$ was the $2 \theta$ peak position obtained from the XRD spectra (see Section 3.4.3). A typical XRD spectrum is shown in Figure 4.5. The single peak occurs at $\approx 34.20^{\circ}$ which closely corresponds to the expected $2 \theta$ peak position of $34.45^{\circ}$ (see Table 3.1) of the (002) reflection present in wurtzite ZnO. Using Bragg's Equation (Equation 3.5) to calculate the $d$ spacing and the relationship between $d$ and $(h, k, l)$ for a hexagonal structure (Equation 3.6) the lattice parameter $c$ can be calculated from the peak position. For this particular XRD spectra the lattice parameter $c$ was calculated as $0.5240 \mathrm{~nm}$ which falls within the values reported in the literature $0.5204-0.5241 \mathrm{~nm}$ for $\mathrm{ZnO}$ [21].

Further evidence confirming that we had indeed deposited $\mathrm{ZnO}$ films came from the UV-visible transmission spectrum as shown in Figure 4.6, showing a high transmitted intensity above the cut-off wavelength. No thickness fringes can be seen in the transmission spectrum, as this was a very thin layer $(\approx 100 \mathrm{~nm}$ as measured by the Dektak). The transmission spectrum was also used to estimate the bandgap of the material by plotting the absorption coefficient vs. photon energy (see Section 3.4.2), as shown in Figure 4.7. For this film the bandgap was estimated to be $\approx 3.27 \mathrm{eV}$ which is close to reported values of $3.30-3.437 \mathrm{eV}$ for $\mathrm{ZnO}$, but on the lower end of the scale $[55,56]$. 


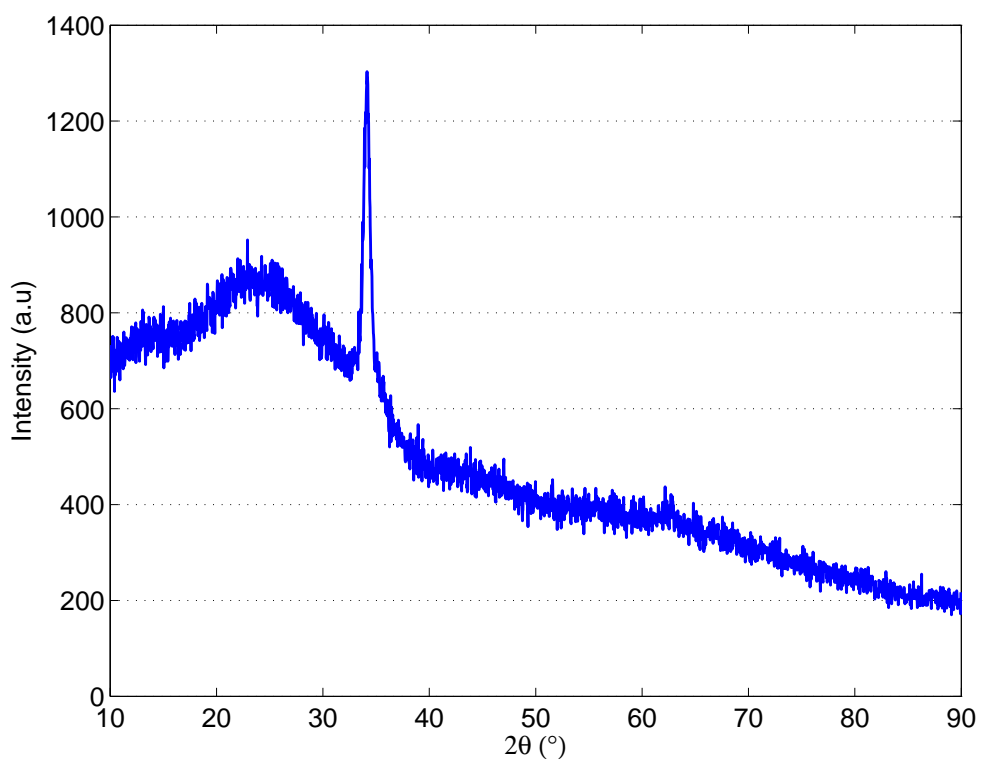

Figure 4.5: XRD spectrum of one of the first $\mathrm{ZnO}$ films deposited where the position of the peak suggests the (002) reflection present in $\mathrm{ZnO}$.

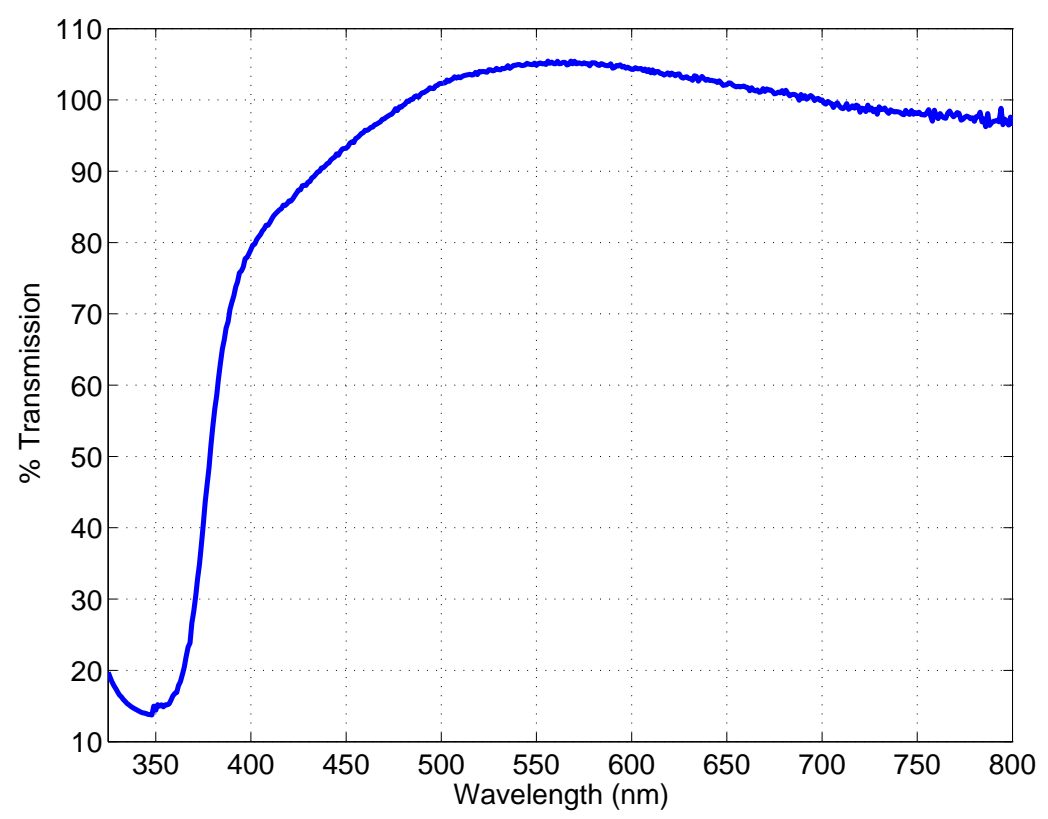

Figure 4.6: UV-visible transmission spectrum of a $\mathrm{ZnO}$ film. 


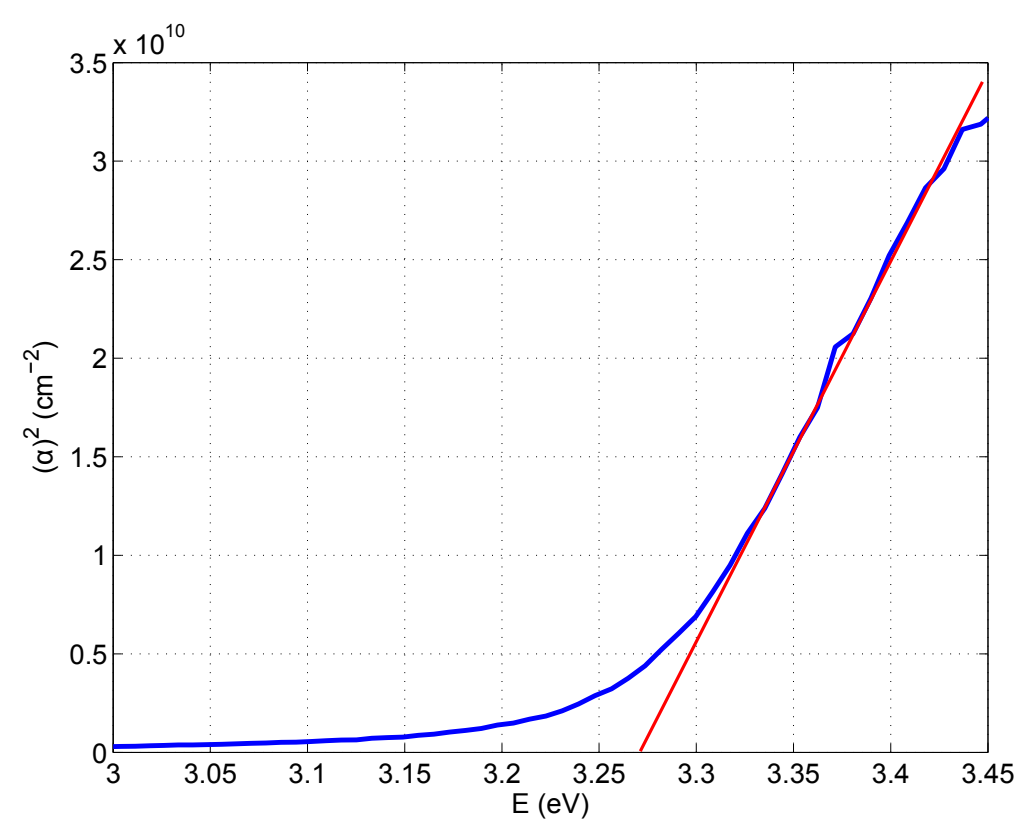

Figure 4.7: Estimating the bandgap from a plot of the absorption coefficient vs. photon energy, of which was $\approx 3.27 \mathrm{eV}$.

In Figure 4.8, a conventional optical photograph of a $\mathrm{ZnO}$ film deposited onto a glass substrate can be seen, showing a transparent film.

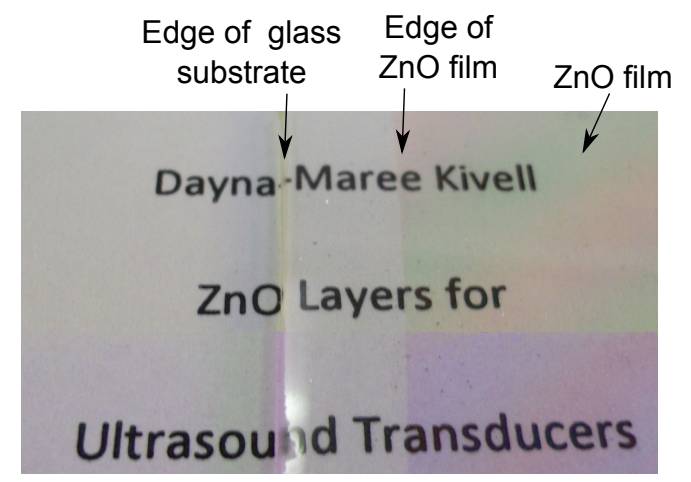

Figure 4.8: Shows the transparency of a $\mathrm{ZnO}$ deposited film.

The above results confirm $\mathrm{ZnO}$ of (001) texture was deposited. Selected deposition parameters were then varied systematically to study their influence on film quality. 


\subsubsection{Effects of RF power}

The influence of the RF power on crystal quality was investigated at three RF powers: $180 \mathrm{~W}, 200 \mathrm{~W}$ and $220 \mathrm{~W}$ and deposited at an Ar flow of $5 \mathrm{sccm}$. The resulting XRD spectra are shown in Figure 4.9. The peak positions indicate the films are (001) orientated, with peak degradation occurring as RF power increases.

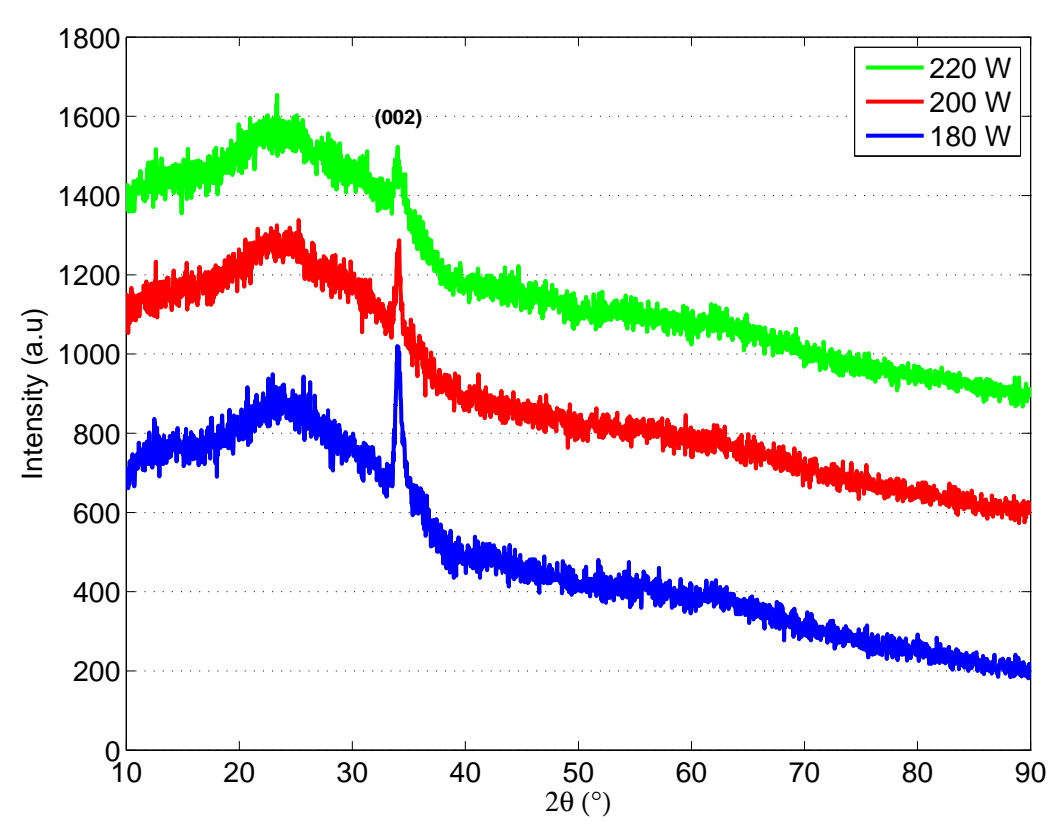

Figure 4.9: XRD spectra as a function of RF power. There is degradation of the (002) peak with increasing RF power. All other parameters held constant: Ar = $5 \mathrm{sccm}$, substrate holder stationary and sputtering time $=40 \mathrm{mins}$.

\subsubsection{Effects of Ar flow}

The effect of Ar flow on growth rate and film quality was investigated with the growth of a series of three films using an RF power of $180 \mathrm{~W}$. The XRD spectra obtained are shown in Figure 4.10. All of the films were (001) orientated. The film deposited at $5 \mathrm{sccm}$ had the best crystal quality as indicated by a low FWHM value, whereas depositions at $3.5 \mathrm{sccm}$ 
and $10 \mathrm{sccm}$ had increased FWHM values and lower (002) peak intensities indicating poorer crystal alignment.

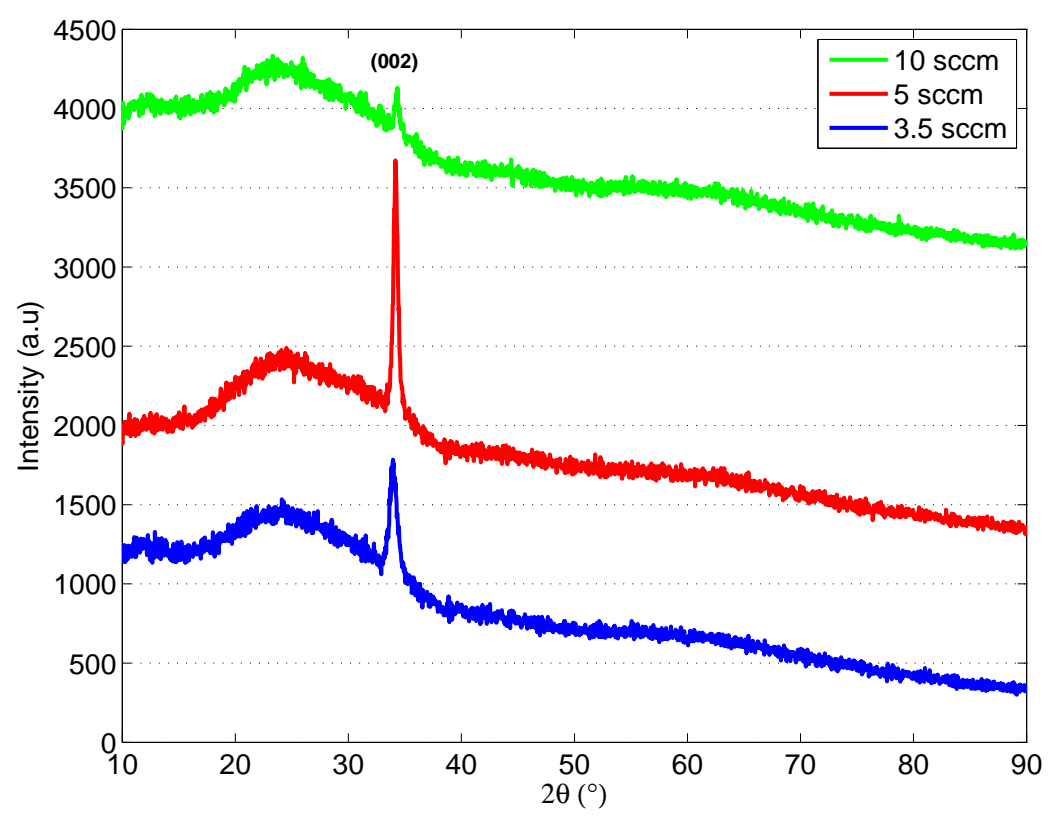

Figure 4.10: XRD spectra for various argon flows. All other parameters held constant: RF power $=180 \mathrm{~W}$, substrate holder rotating, at Ar flow $=3.5,5$ and $10 \mathrm{sccm}$ chamber pressures were $1.3 \times 10^{-2}, 2.5 \times 10^{-2}$ and $6.0 \times 10^{-2} \mathrm{mbar}$ respectively.

The observed trend between the deposition rate and Ar flow was plotted and is shown in Figure 4.11. These results were consistent with that from Section 4.1, indicating that maximum deposition rates are achieved when depositing at an Ar flow between $\approx 4-6 \mathrm{sccm}$. 


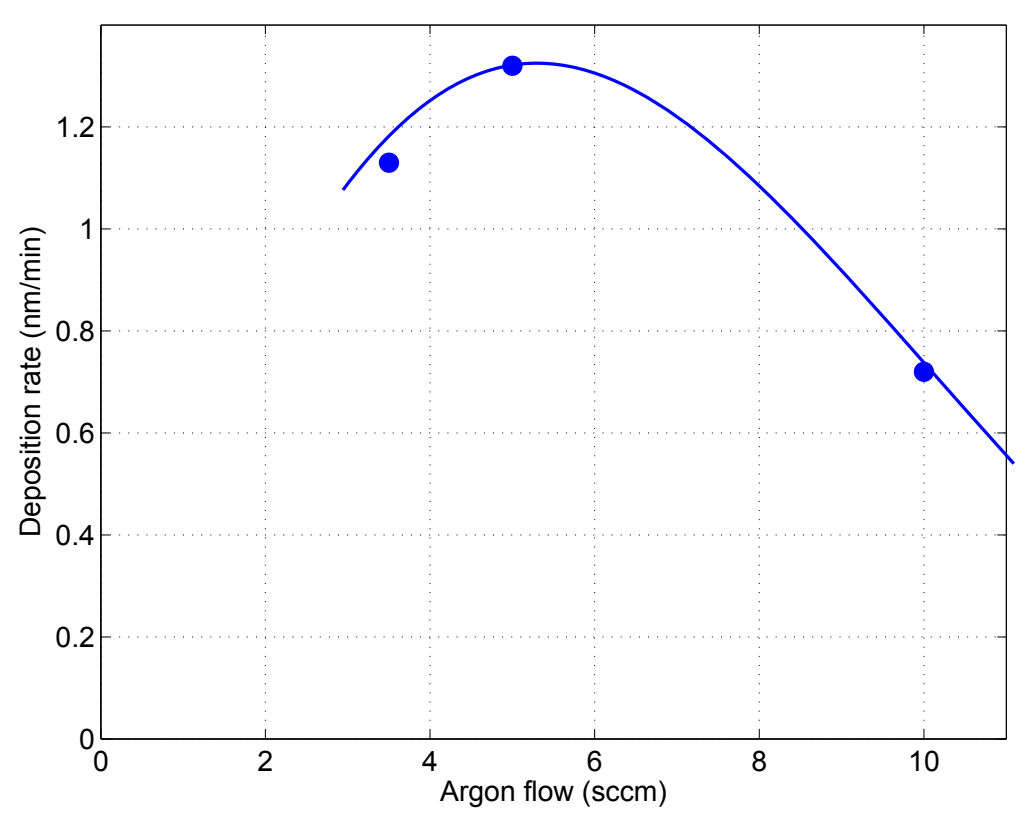

Figure 4.11: Deposition rates as a function of the three Ar gas flows. The trend observed is similar to that seen in Section 4.1 suggesting that deposition rate is the highest between $\approx 4-6 \mathrm{sccm}$.

\subsubsection{Effects of substrate temperature}

It was found that a maximum substrate temperature of $\approx 100^{\circ} \mathrm{C}$ could be reached with heating from the quartz lamp. The influence of substrate heating was investigated at this temperature by comparing the deposition of a film with no substrate heating (termed ambient) to one deposited at a substrate temperature of $100^{\circ} \mathrm{C}$. Figure 4.12 compares the XRD spectra of these two films. Both films are (001) orientated, with the heated substrate resulting in a higher peak intensity. The deposition rate during the ambient substrate temperature deposition was $\approx 1.4 \mathrm{~nm} / \mathrm{min}$, much higher than when depositing at $100^{\circ} \mathrm{C}$ which was $\approx 0.7 \mathrm{~nm} / \mathrm{min}$.

A curious point to note about these spectra is the asymmetrical broadening of the tails, more noticeable in the deposition at $100^{\circ} \mathrm{C}$. This may be the result of the $(101)$ orientation at $\approx 36^{\circ}$ coming through and broadening 
the tail.

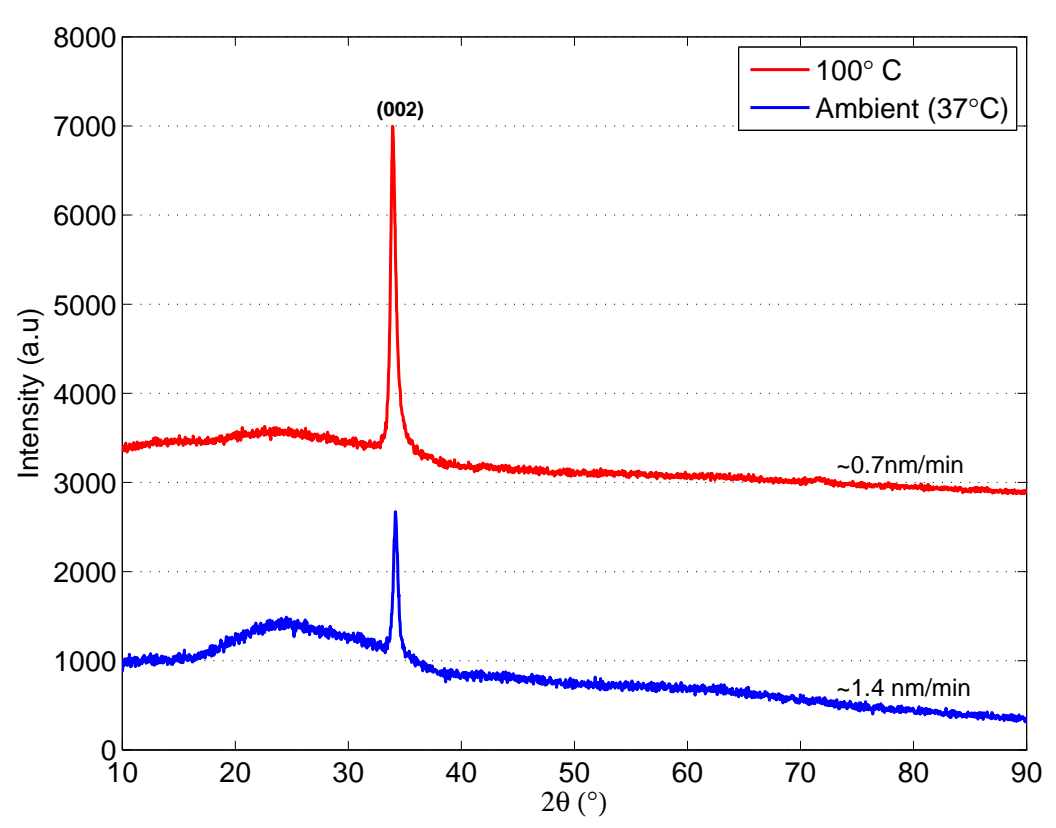

Figure 4.12: The influence of substrate heating on crystal structure. All other parameters held constant: RF power $=180 \mathrm{~W}, \mathrm{Ar}=6 \mathrm{sccm}$, substrate holder rotating, and substrate temperature at ambient $37^{\circ} \mathrm{C}$ and at $100^{\circ} \mathrm{C}$ with heating.

\subsubsection{Effects of substrate rotation}

Initial experiments indicated low deposition rates when using a rotating substrate holder. This motivated a more in depth analysis between depositions performed with a rotating substrate holder and a stationary substrate holder. The results were evaluated in terms of:

(a) Deposition rate (it was expected that depositing with a stationary substrate holder to be higher).

(b) Uniformity (it was expected that depositing with a rotating substrate holder to be more uniform). 
The film uniformity was investigated by depositing $\mathrm{ZnO}$ onto eight glass substrates, as configured in Figure 4.13a, and performing depositions with both a rotating and stationary substrate holder. In this figure the position of the $\mathrm{ZnO}$ target has been indicated because it would be expected that during a stationary holder deposition substrates closer to the target will experience a higher deposition rate. A cross section of this arrangement is shown in Figure 4.13b, where it becomes more obvious how the substrates are positioned with respect to the $\mathrm{ZnO}$ target.

The film thickness was measured at three different points across each substrate using the Dektak (described in Section 3.5) and the deposition rates calculated. These points were at positions labelled inner $(\approx 40 \mathrm{~mm})$, centre $(\approx 75 \mathrm{~mm})$ and outer $(\approx 105 \mathrm{~mm}$ from the centre of the holder), indicated on slide \#5 from Figure 4.13a. At each of these positions XRD was also performed so that film quality could also be related to a deposition rate.

The variation in deposition rates across each of the eight substrates for the two different types of depositions are shown in Figure 4.14. Relatively uniform rates of $\approx 1.0$ to $1.5 \mathrm{~nm} / \mathrm{min}$ were achieved when the substrate holder was rotating. However, with a stationary substrate holder very poor uniformity occurred. Slides 2, 3 and 4 experienced higher deposition rates $\approx 1.7$ to $4 \mathrm{~nm} / \mathrm{min}$. This was the result of these slides being positioned closer to the target and therefore closer to the peak density of the plasma. Slides 6,7 and 8 being furthest away had very low growth rates reaching a maximum of $\approx 0.8 \mathrm{~nm} / \mathrm{min}$. 


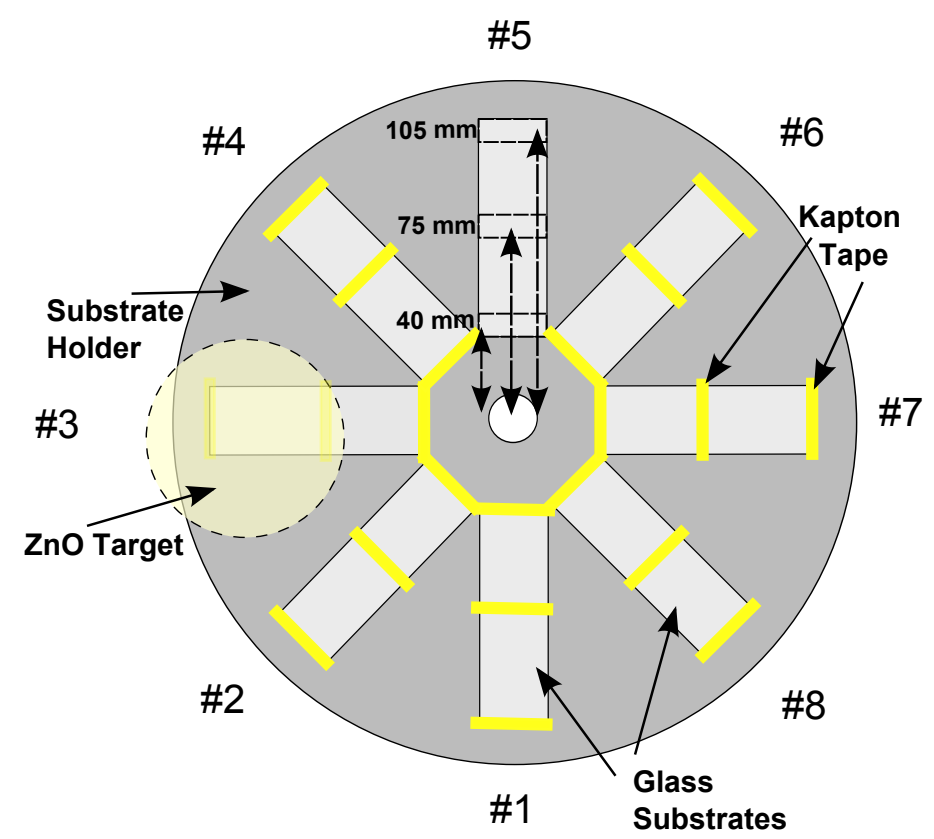

(a) Eight glass substrates fixed to the substrate holder using Kapton tape. Thickness measurements were performed at $\approx 40,75$ and $105 \mathrm{~mm}$ from the centre of the substrate holder.

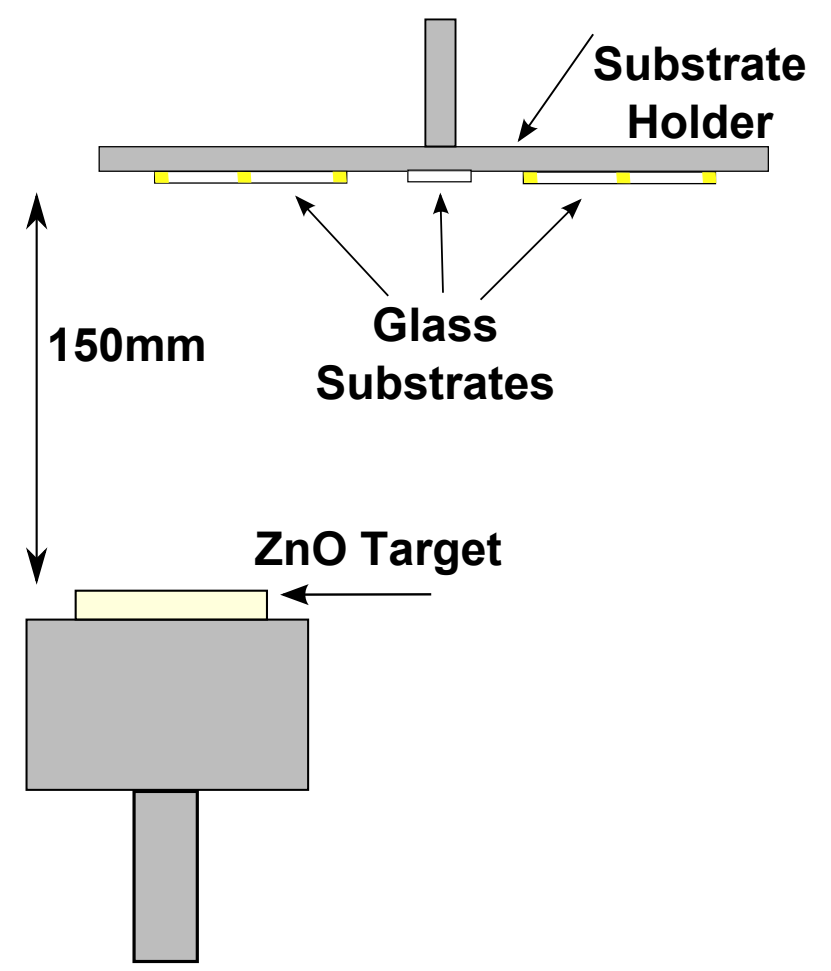

(b) Substrate positioning in relation to the $\mathrm{ZnO}$ target.

Figure 4.13: Configuration of the substrates with reference to the $\mathrm{ZnO}$ target, an important characteristic during the stationary holder deposition due to an expected higher deposition rate on films closer to the target. 


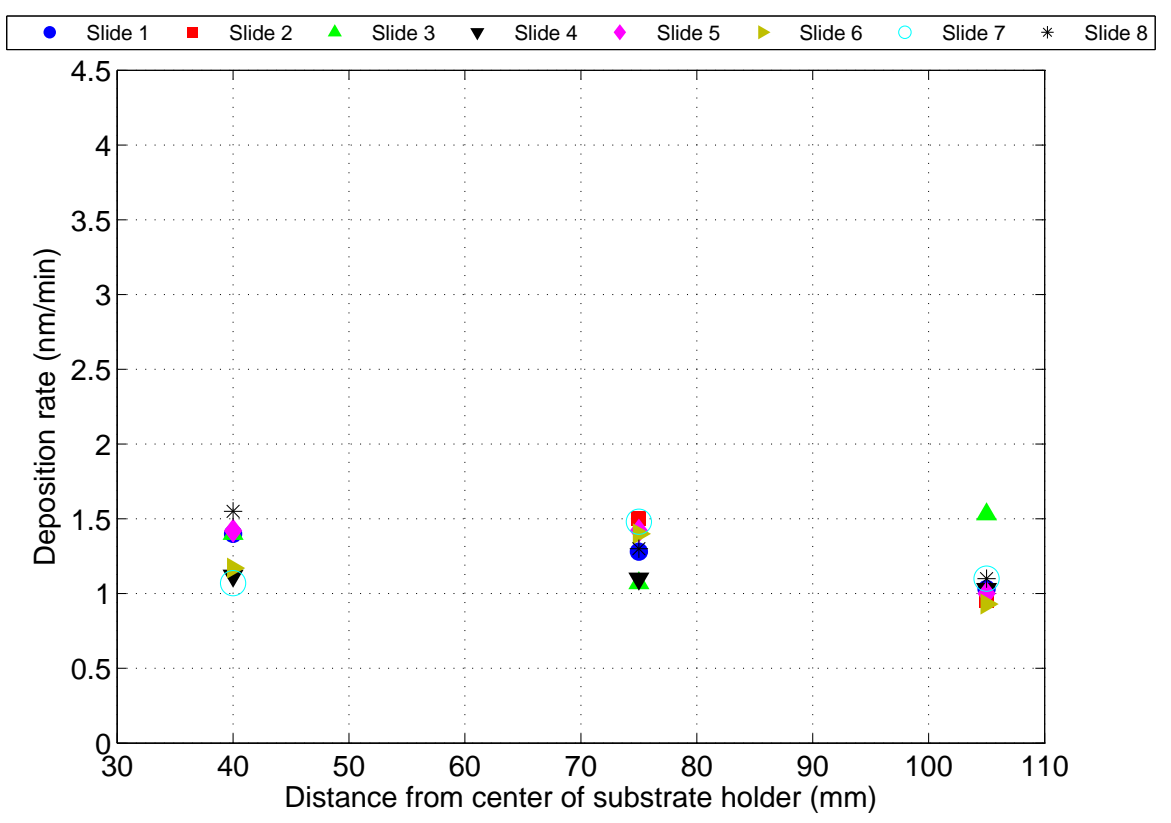

(a)

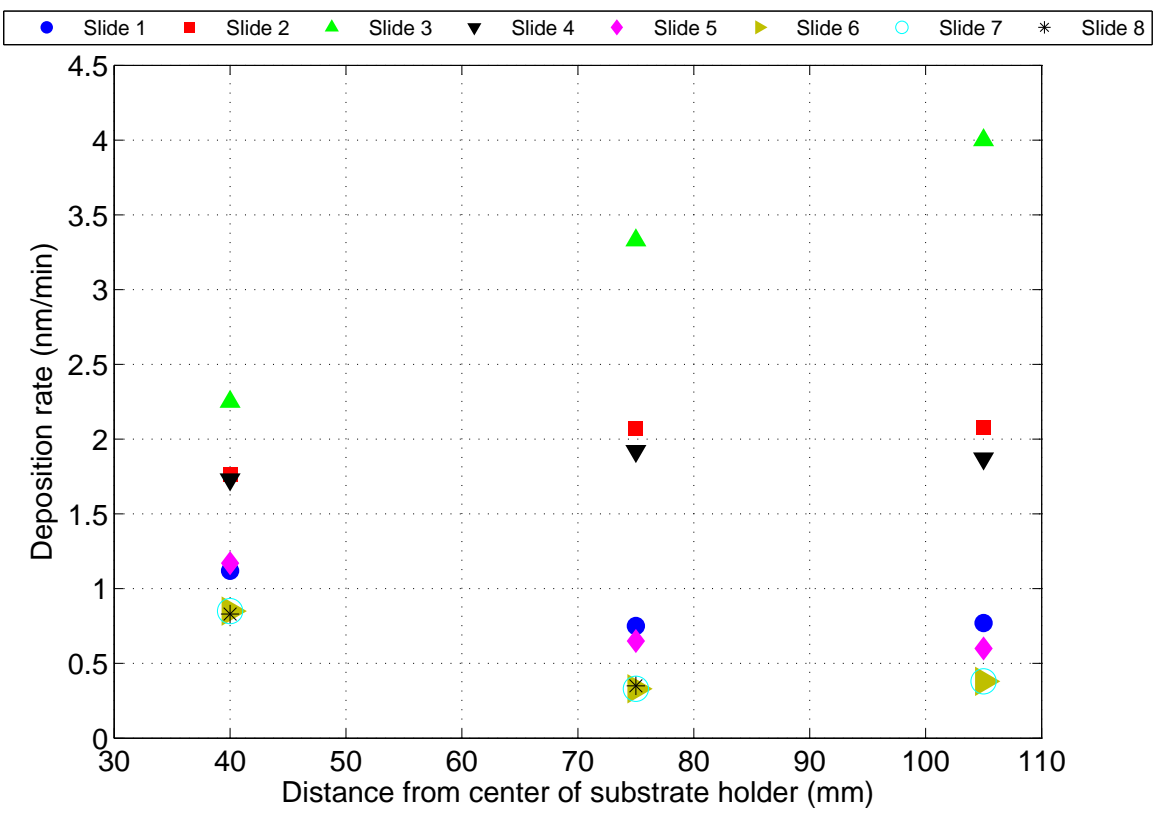

(b)

Figure 4.14: Distribution of growth rates observed across two depositions with eight slides used as substrates in each deposition. In (a) The substrate holder was rotated at $35 \mathrm{rpm}$, while in (b) The substrate holder was stationary. 
The crystalline quality of these films were analysed using XRD. Figure 4.15 shows the XRD spectra for the rotating substrate holder deposition at deposition rates of $\approx 1.1$ and $\approx 1.4 \mathrm{~nm} / \mathrm{min}$. In both regions the films were (001) oriented with low peak broadening suggesting good crystal alignment.

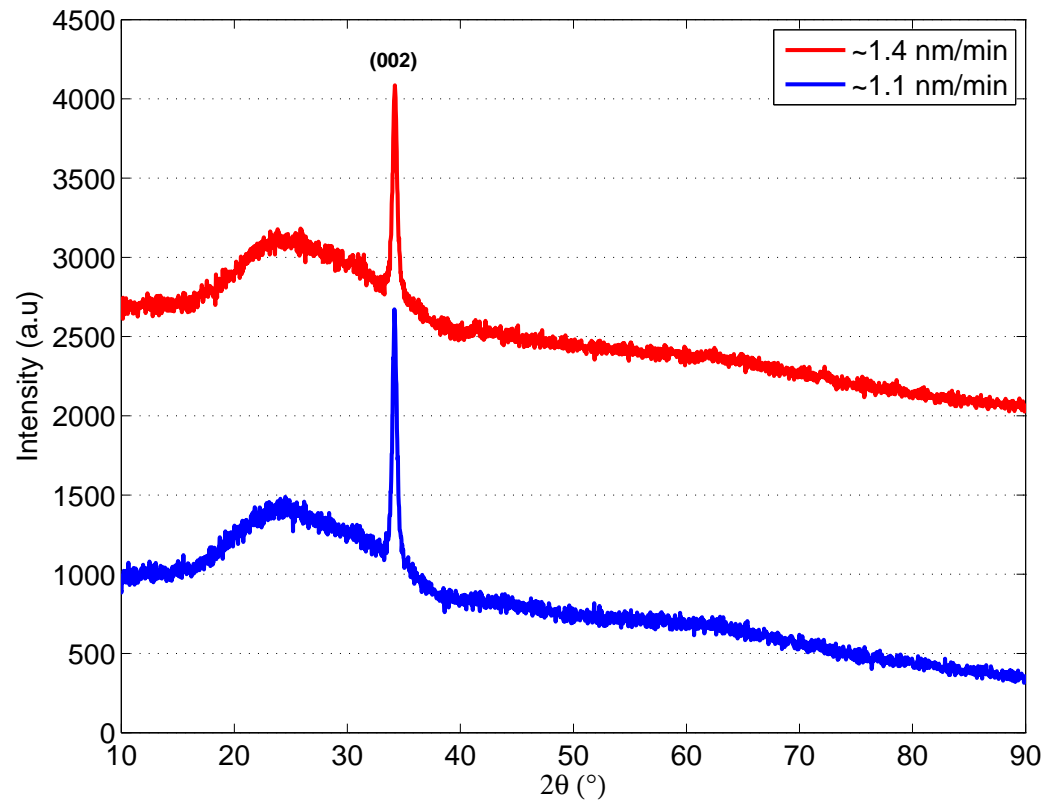

Figure 4.15: Crystal quality results at deposition rates of $\approx 1.1$ and $\approx 1.4 \mathrm{~nm} / \mathrm{min}$ with a rotating substrate holder. All other parameters held constant: RF power = $180 \mathrm{~W}, \mathrm{Ar}=5 \mathrm{sccm}$, and sputtering time $=60 \mathrm{mins}$.

Figure 4.16 shows the XRD spectrums across slides 2, 3 and 4 at the approximate 40,75 and $105 \mathrm{~mm}$ measurement points for the stationary holder deposition. All films showed (001) orientation as indicated by the peak position. In Figure 4.17 the FWHM as measured for the (002) reflection is plotted against the deposition rate as estimated by the Dektak thickness measurements. Films grown at slower deposition rates had better crystal quality as indicated by lower FWHM values. Slide 3, positioned directly above the target, had the highest growth rate and thus the thickest films, but also showed severe degradation in film quality as indicated by the very broad (002) XRD peaks. 


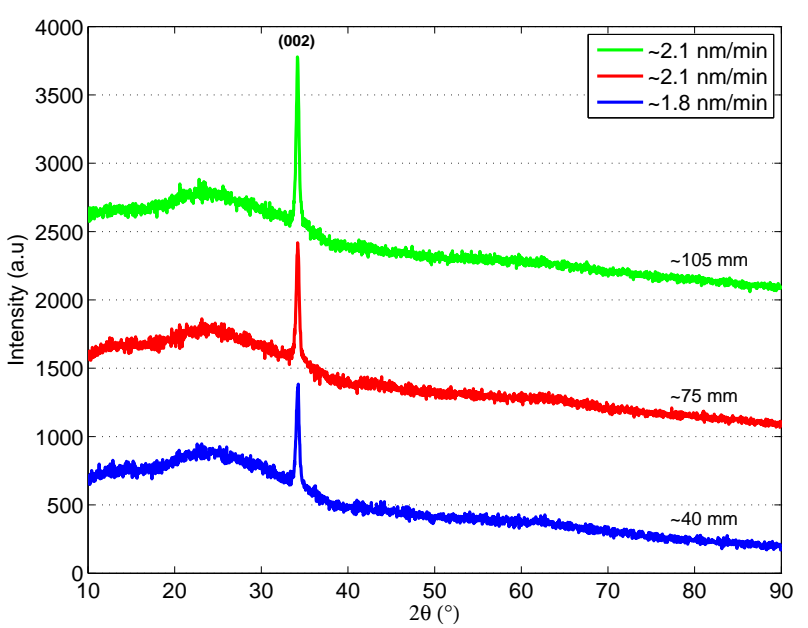

(a)

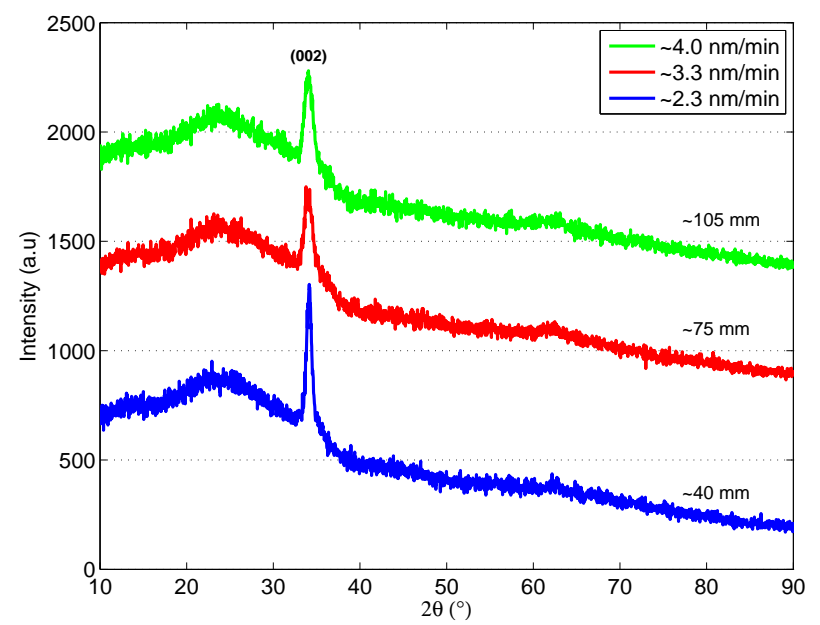

(b)

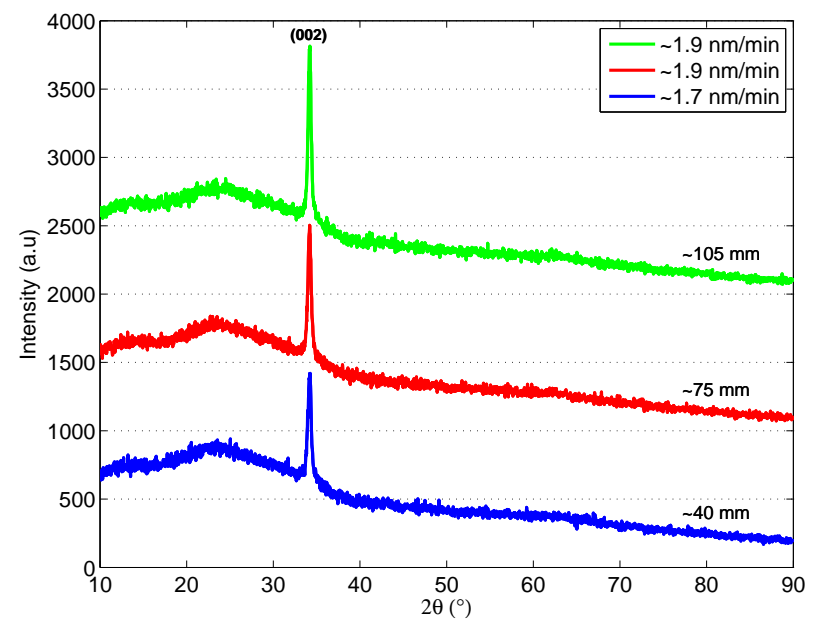

(c)

Figure 4.16: XRD crystal quality as a function of deposition rate with a stationary substrate holder: (a) Slide \#2, (b) Slide \#3 and (c) Slide \#4. Measurements were performed at $\approx 40,75$ and $105 \mathrm{~mm}$ from the centre of the substrate holder. All other parameters held constant: $\mathrm{RF}$ power $=180 \mathrm{~W}, \mathrm{Ar}=5 \mathrm{sccm}$, and sputtering time $=60$ mins . 


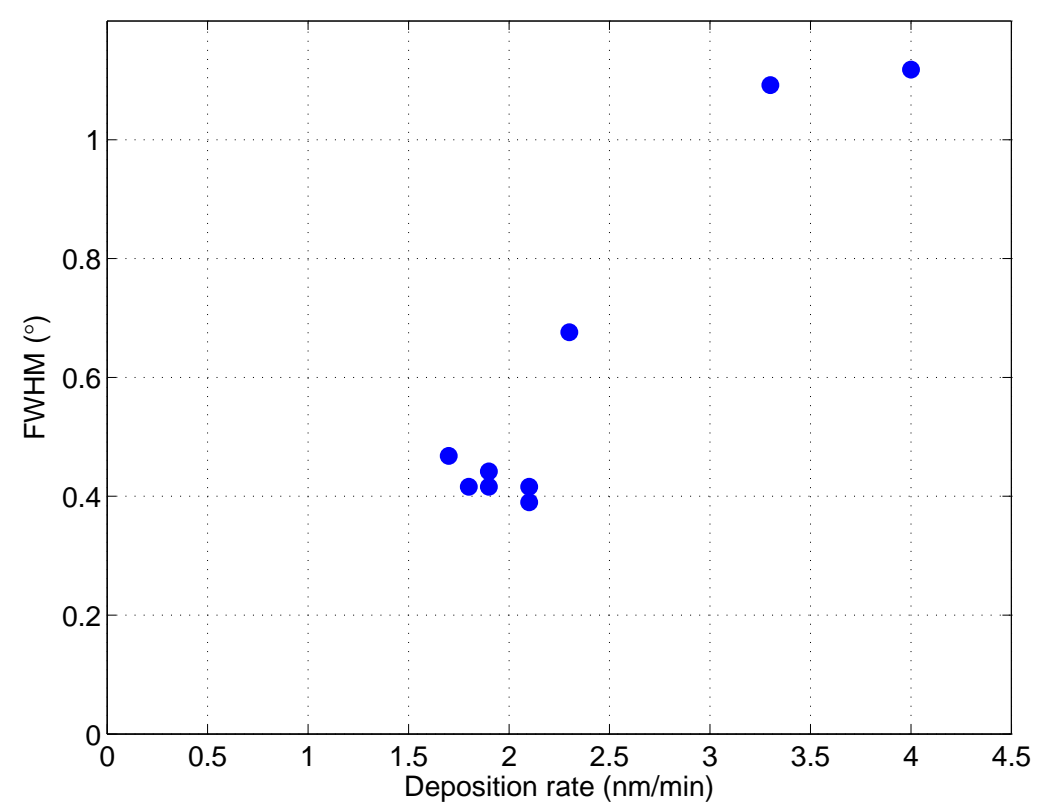

Figure 4.17: The (002) FWHM for slides 2, 3 and 4 from the stationary holder deposition as a function of deposition rate.

\subsubsection{Conclusions from initial system evaluation}

- The initial evaluation of the HHV Auto500 indicates that the system can successfully be used to deposit $\mathrm{ZnO}$ films by means of RF magnetron sputtering. The deposition rate was found to be primarily controlled by the RF power and the Ar gas flow. In the case of RF power a linear increase in growth rate with power is observed, but in the case of Ar flow a maximum in the growth rate occurred between flow rates of $4-6 \mathrm{sccm}$. This trend appeared to be independent of RF power.

- The film uniformity, deposition rate and crystal quality was investigated using both a rotating and stationary substrate holder. The rotating substrate holder produced good uniformity across all substrates, but at a very low growth rate. In the case of a stationary sub- 
strate holder the deposition rates were higher however there was a larger variation in deposition rates found across the substrates depending on their relative position to the $\mathrm{ZnO}$ target.

- From XRD results it is clear that all films show the desired (001) orientation. However the poorest crystalline quality (as indicated by higher FWHM values) coincides with the areas of highest deposition rates.

- In all the depositions performed thus far the deposition rates achieved were much lower than those desired for this application. In order to increase the deposition rate it was decided to modify the sputter chamber by adding an adjustable shaft for the substrate holder. This would allow the target-to-substrate distance to be decreased from the fixed $150 \mathrm{~mm}$ in the original system.

\subsection{Chamber modification \#1: Reduce target-to- substrate distance}

The new shaft extension was $\approx 95 \mathrm{~mm}$ in length allowing variable targetto-substrate distances of $150 \mathrm{~mm}$ to $75 \mathrm{~mm}$ in $15 \mathrm{~mm}$ increments. Figure 4.18 shows the new chamber geometry with the substrate holder lowered to $\approx 75 \mathrm{~mm}$ above the target. This minimum distance of $75 \mathrm{~mm}$ was limited by the locations of the quartz lamp and gas inlet. This section will first discuss the influence of the target-to-substrate distance on the observed deposition rate. It will then consider the influence of the sputtering parameters on the deposited $\mathrm{ZnO}$ film under the new chamber geometry. Due to the large number of permutations of sputtering parameters, every case could not be investigated but selected values of substrate temperature and RF power were considered as these were deemed the most critical factors for producing thin films to our requirements. 


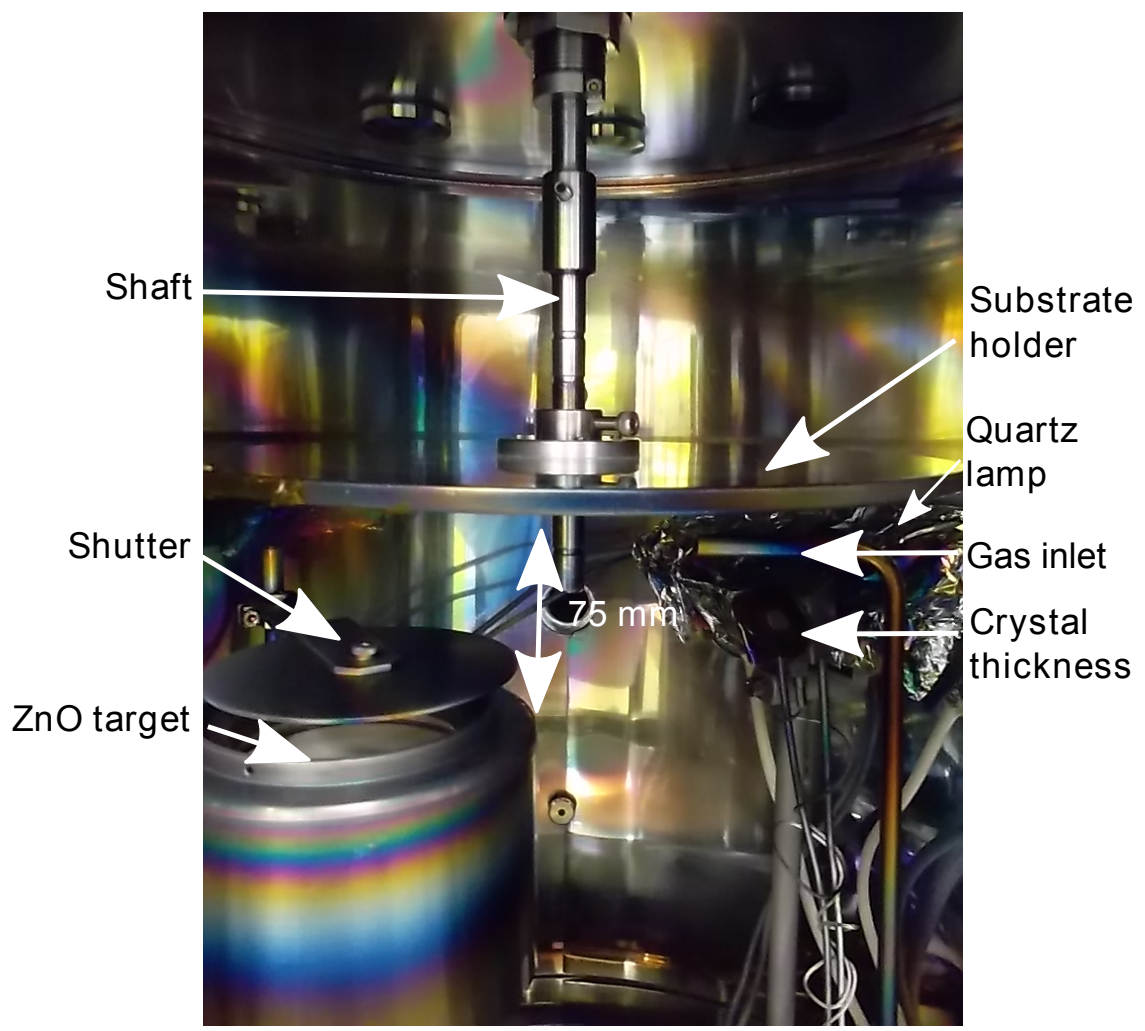

Figure 4.18: Inside the sputter coater chamber with the substrate holder attached to the shaft at a new substrate-to-target distance of $75 \mathrm{~mm}$. Aluminium foil is covering the quartz lamp to prevent deposited layers building up on the surface.

\subsubsection{Effect of reduced target-to-substrate distance on de- position rate}

With the new shaft extension in place, an investigation into the achievable deposition rates at various target-to-substrate heights was performed, using both a rotating and a stationary substrate holder.

\subsubsection{Rotating substrate holder}

A series of three depositions were performed at target-to-substrate distances of $75 \mathrm{~mm}, 95 \mathrm{~mm}$ and $150 \mathrm{~mm}$, and the thickness of the films mea- 
sured. The calculated deposition rates are presented in Figure 4.19. As would be expected when using a rotating substrate holder the films are relatively uniform. As the distance between the target and substrate decreases, the deposition rate increases. When the holder was at the lowest substrate position $(75 \mathrm{~mm})$ the deposition rate was double that of when it was at the highest position $(150 \mathrm{~mm})$. As the highest deposition rate achieved was only $\approx 7 \mathrm{~nm} / \mathrm{min}$, this was still well below the minimum required deposition rate of $>17 \mathrm{~nm} / \mathrm{min}$.

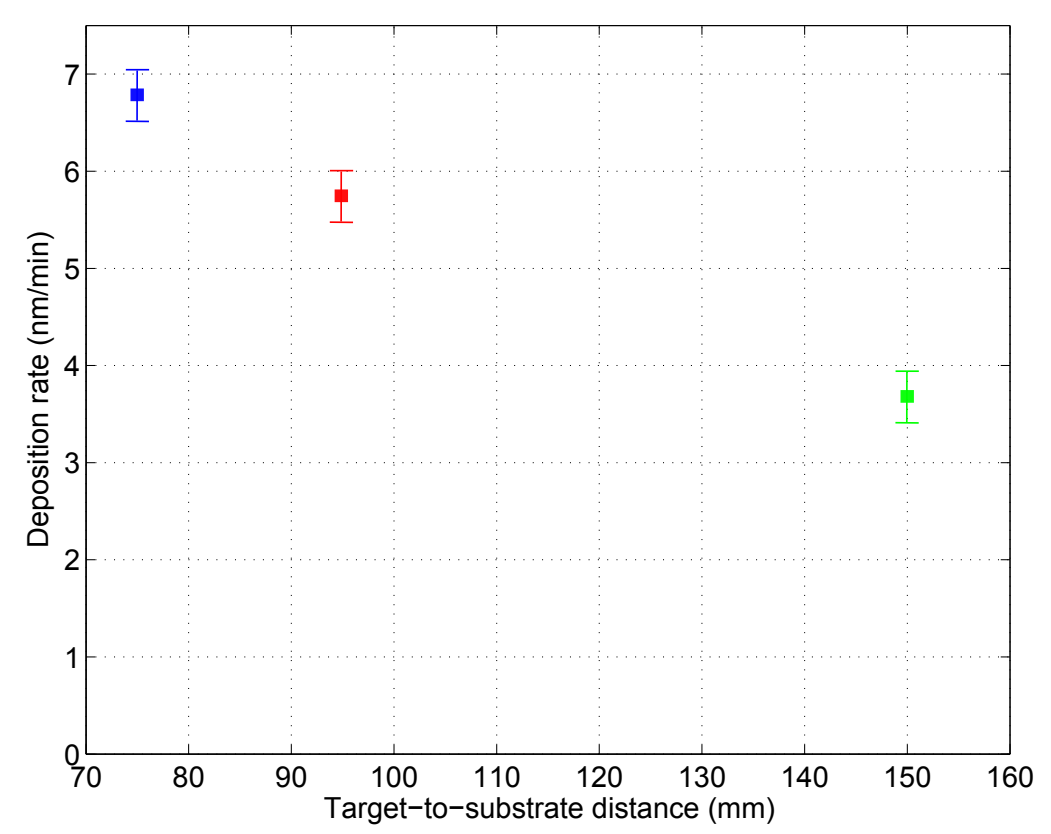

Figure 4.19: Influence of substrate-to-target distance on deposition rate for a rotating substrate holder. All other parameters held constant: RF power $=240 \mathrm{~W}$, $\mathrm{Ar}=6 \mathrm{sccm}$, no external heating and sputtering time $=60 \mathrm{mins}$.

As in previous results, the deposition rate could potentially be further increased by using a stationary holder and positioning the substrates above the $\mathrm{ZnO}$ target. 


\subsubsection{Stationary substrate holder}

As the highest deposition rates were obtained at a target-to-substrate distance of $75 \mathrm{~mm}$, the lowest position possible, this was the height the stationary substrate holder was positioned. Three substrates were then attached to the holder, as shown in Figure 4.20 and a deposition performed. Film thicknesses were measured and the deposition rate calculated for inner $(\approx 40 \mathrm{~mm})$, centre $(\approx 75 \mathrm{~mm})$ and outer $(\approx 105 \mathrm{~mm})$ positions across the three substrates. These results are compared in Figure 4.21 to the results obtained with a rotating substrate at the same target-to-substrate distance. With a stationary holder the deposition rate is considerably higher than with a rotating holder, this is because the samples are held above the $\mathrm{ZnO}$ target and there is an almost direct line of sight for the sputtered $\mathrm{Zn}$ and $\mathrm{O}$ atoms to travel and reach the substrate surface. A rate as high as $\approx 34 \mathrm{~nm} / \mathrm{min}$ was obtained on the outer edge of the central substrate (directly above the target). However, it is also obvious from Figure 4.21 that the stationary substrates lead to poor uniformity in deposition rates across the substrates.

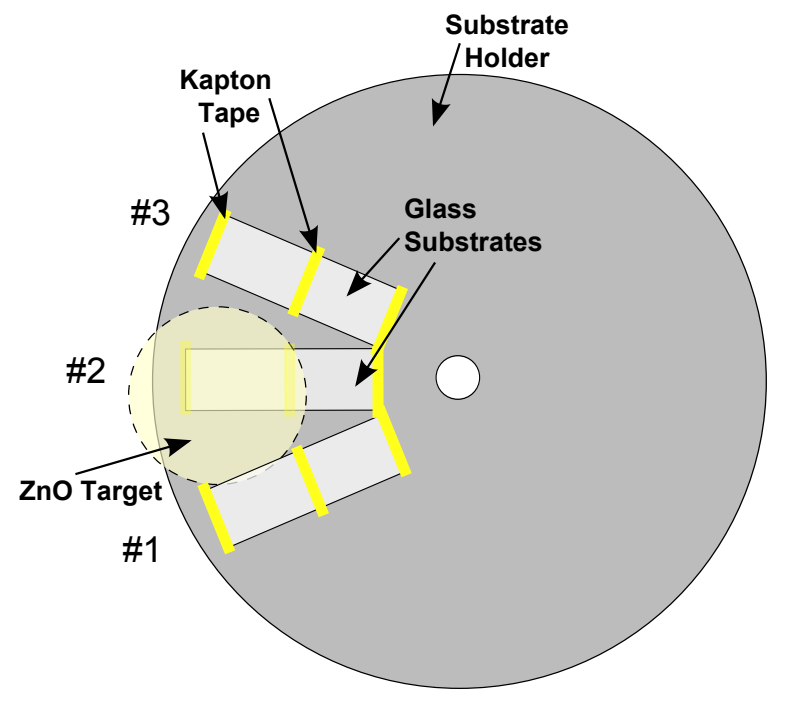

Figure 4.20: The configuration of the three samples on substrate holder with respect to $\mathrm{ZnO}$ target position. 


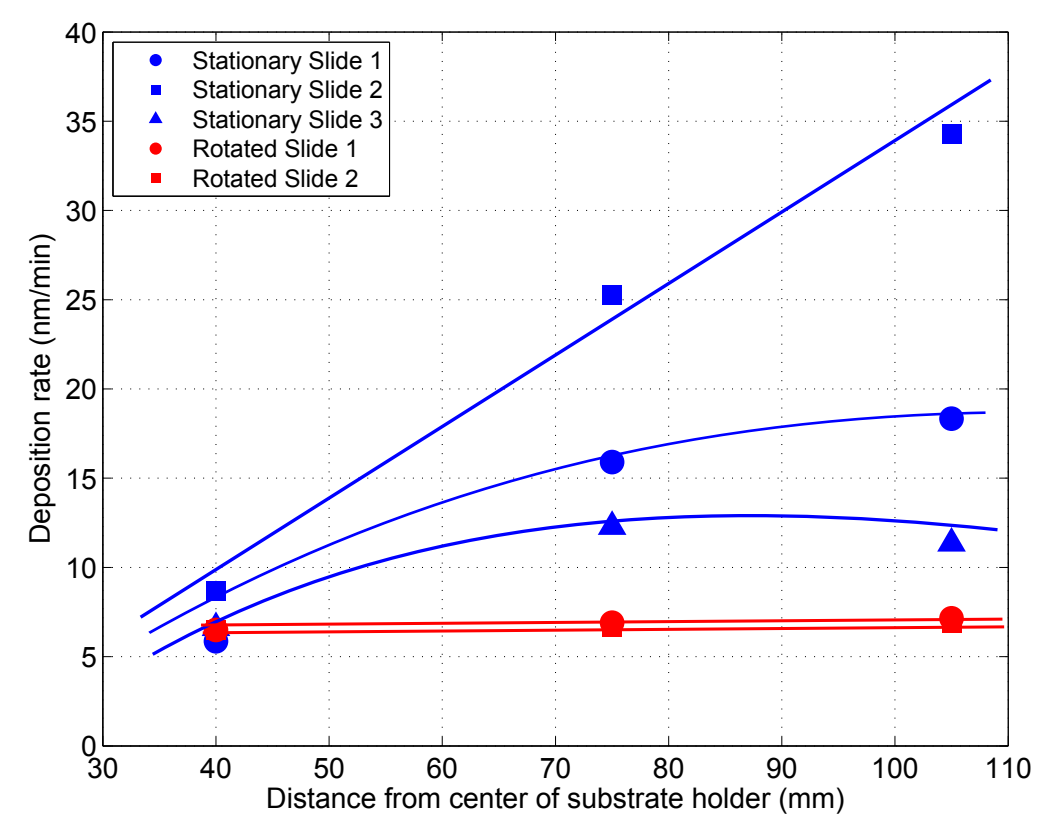

Figure 4.21: Comparing deposition rates between a stationary and rotating substrate holder. All other parameters held constant: RF power $=240 \mathrm{~W}, \mathrm{Ar}=6 \mathrm{sccm}$, no external heating and sputtering time $=60$ mins.

The XRD spectra of the three substrates using a stationary substrate holder are shown in Figure 4.22. For each XRD spectra the approximate growth rate at that point is also indicated.

It is clear from the XRD spectra that the films are not always purely caxis oriented. It would appear that a c-axis orientation is only observed for the lower deposition rates with extra orientations appearing in the diffraction pattern at higher deposition rates. The new reflections were identified as the (100), (101), (103) and (110) planes. As these new orientations increased in intensity, the (002) peak also dramatically decreased in intensity. At the point of maximum deposition rate $(\approx 34 \mathrm{~nm} / \mathrm{min})$ the $(100)$ is the strongest reflection visible. This would indicate that the preferred c-axis orientation is not maintained at these high deposition rates, but that the film becomes polycrystalline containing mixed grains of (100), (001), (101) and (110) orientations. 


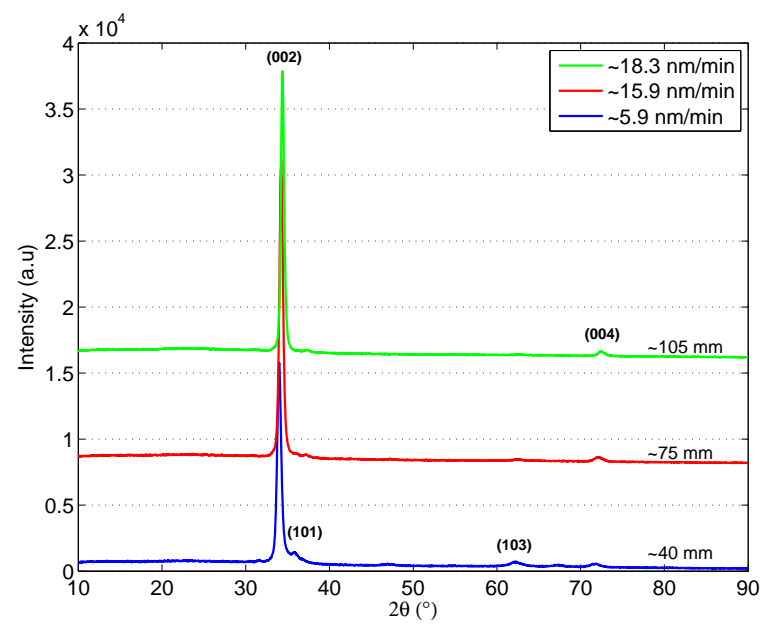

(a)

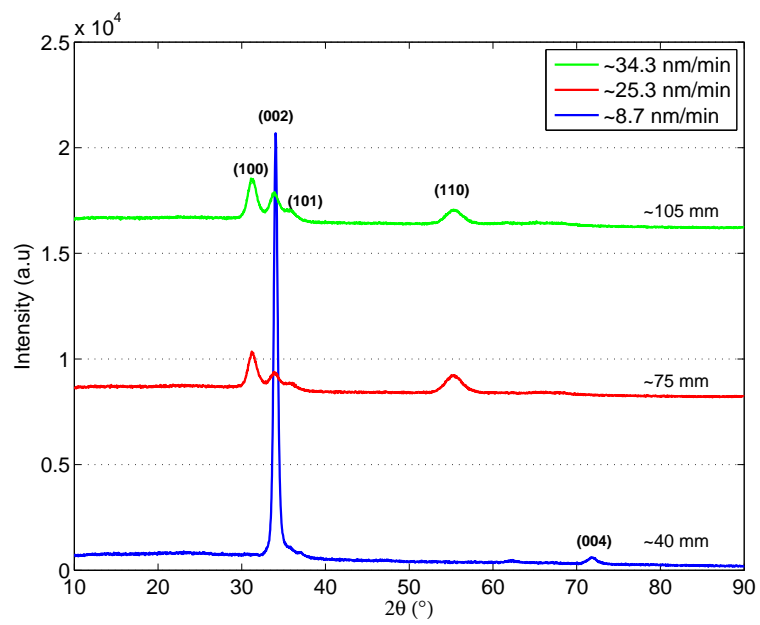

(b)

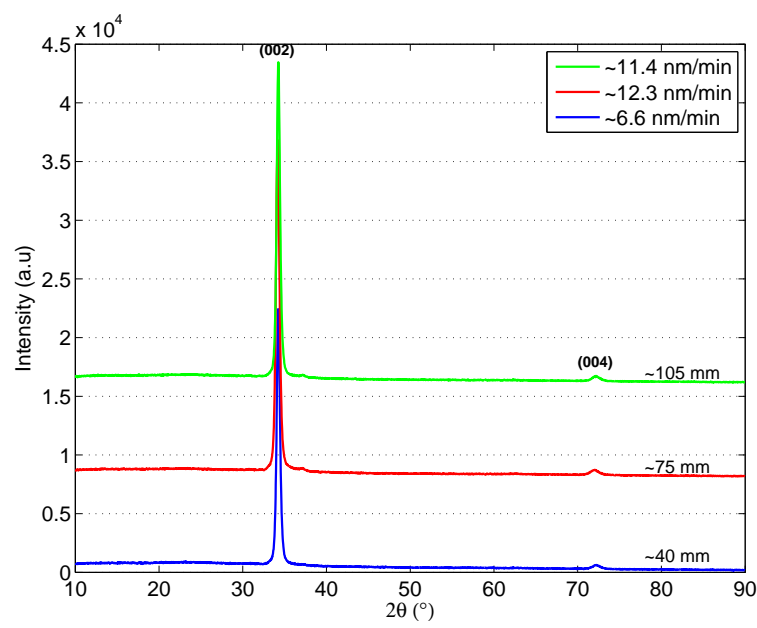

(c)

Figure 4.22: Crystal quality as a function of deposition rate with a stationary substrate holder: (a) Slide \#1, (b) Slide \#2 and (c) Slide \#3. All other parameters held constant: $\mathrm{RF}$ power $=240 \mathrm{~W}, \mathrm{Ar}=6 \mathrm{sccm}$, no external heating and sputtering time $=60$ mins . 
Figure 4.23 shows the ratio of the (002) peak intensity to the (100) peak intensity. This figure represents the change in film orientation as deposition rate increases. It indicates that only when depositing at low deposition rates $(<15 \mathrm{~nm} / \mathrm{min}$ ) does the film maintain (001) preferential orientation. As soon as the deposition rate exceeded this value, competitive orientations were formed in the crystal structure. At the highest deposition rates the (001) orientation nearly disappears from the film.

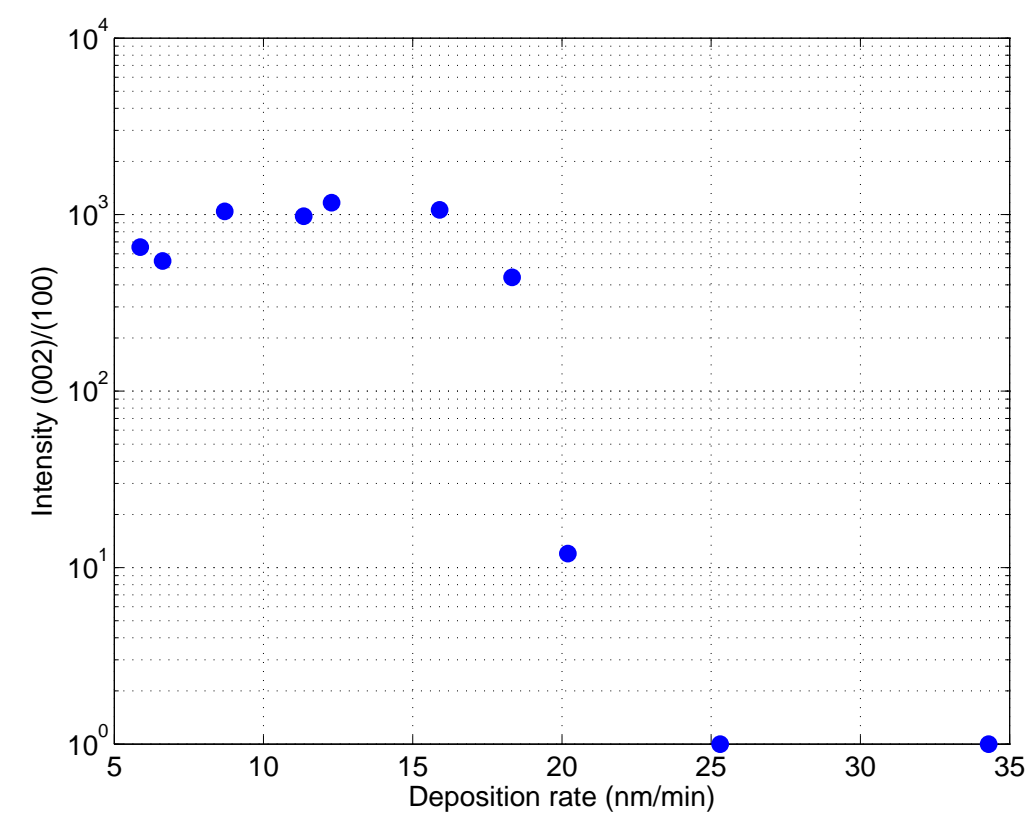

Figure 4.23: The ratio between the (002) and (100) peak intensities as a function of deposition rate. As the deposition rate increases past $\approx 15 \mathrm{~nm} / \mathrm{min}$ the film is no longer (001) preferentially oriented.

The results from using a smaller target-to-substrate distance clearly show that higher deposition rates could be obtained. In the case of a stationary substrate holder, placed directly above the target, deposition rates within the required range could be obtained. However, it was also clear that the high growth rates led to deterioration in c-axis alignment of the films and that multiple orientations were found.

The aim of the next part of this work was to maintain a high growth 
by using a stationary substrate holder at a target-to-substrate distance of $75 \mathrm{~mm}$, and to study the effect of other deposition parameters such as substrate temperature and RF power on the resultant film orientations and crystal structure. As the position of the central substrate, placed directly above the $\mathrm{ZnO}$ target maintained the highest deposition rate, the sputtering parameters were optimised to grow (001) oriented films at this position. Therefore, unless otherwise stated, results will be displayed from substrates grown at this primary position.

\subsubsection{Effect of substrate temperature}

From the above results it would be expected that (001) oriented films could be deposited at the primary position by decreasing the deposition rate at that point to $<15 \mathrm{~nm} / \mathrm{min}$. However, this was the minimum desired deposition rate. In an effort to preserve the crystalline quality at the higher deposition rates the influence of substrate heating was investigated.

At the substrate height of $75 \mathrm{~mm}$ the substrate holder was closer to the quartz lamp than in the original chamber configuration resulting in a higher maximum substrate temperature of $150^{\circ} \mathrm{C}$ being achieved. To investigate substrate heating a deposition was performed at a substrate temperature of $150^{\circ} \mathrm{C}$ at $240 \mathrm{~W}$. Figure 4.24 compares the XRD spectra of the film deposited at ambient substrate temperature and at $150^{\circ} \mathrm{C}$. At $150^{\circ} \mathrm{C}$ the film does not show (001) preferential growth, with extra planes dominating the orientation of the film. These were identified as the (100), (101), (110), (112) and (201). Similar reflections were found for the deposition performed at ambient temperature, except for the introduction of the (112) and the (201) when substrate heating was applied. 


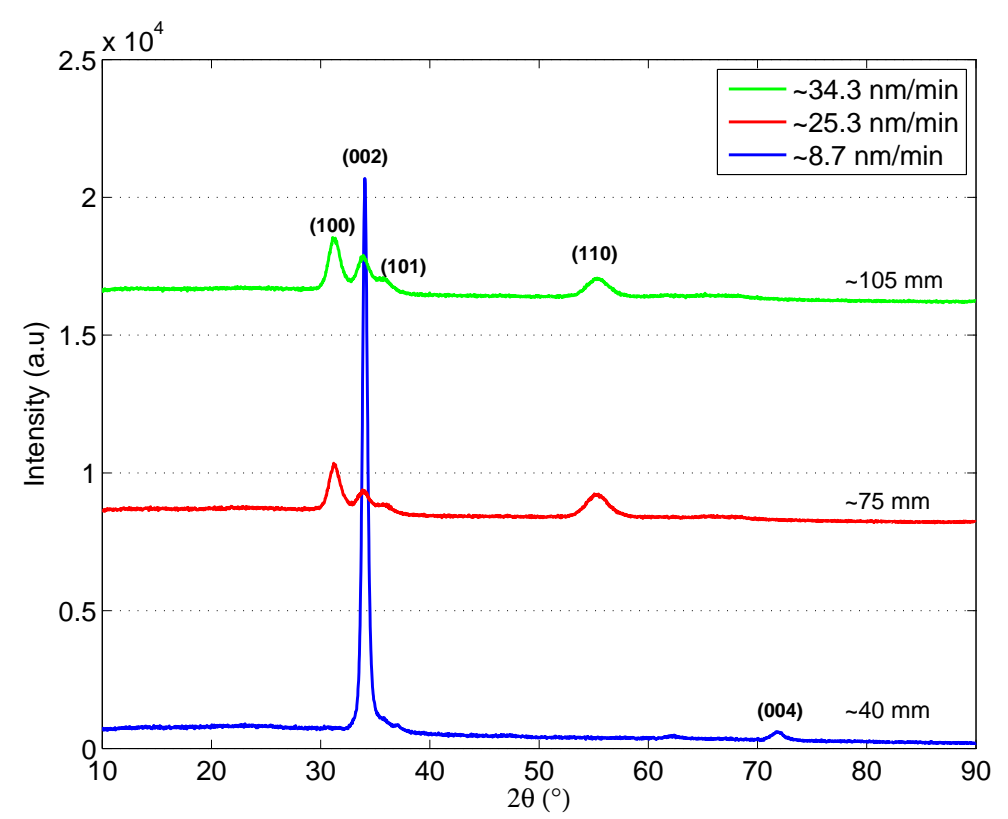

(a)

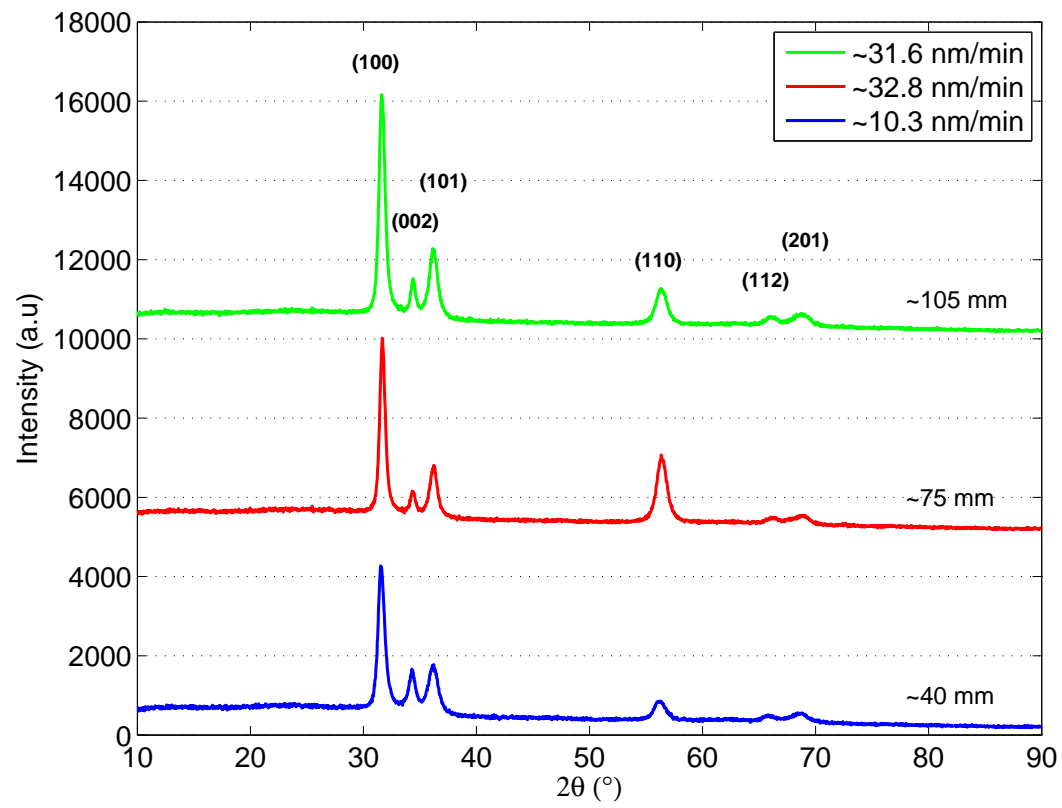

(b)

Figure 4.24: Influence of substrate temperature on crystal quality: (a) Grown at ambient temperature and (b) Grown at $150^{\circ} \mathrm{C}$. All other parameters held constant: $\mathrm{RF}$ power $=240 \mathrm{~W}, \mathrm{Ar}=6 \mathrm{sccm}$ and sputtering time $=60 \mathrm{mins}$. 


\subsubsection{Effects of RF power}

As discussed previously, the deposition rate was found to be linearly dependent on RF power over the range of $180-320 \mathrm{~W}$. In an effort to improve crystalline properties on the central substrate, the deposition rate was reduced by decreasing the RF power. A deposition was performed at $150 \mathrm{~W}$ and the crystal quality and measured deposition rates were compared to the previous deposition at $240 \mathrm{~W}$. Figure 4.25 shows the XRD spectra from the central substrates of each of these two depositions. Depositing at $150 \mathrm{~W}$ produced strong c-axis oriented films at the primary deposition position, reaching a maximum deposition rate of $\approx 15 \mathrm{~nm} / \mathrm{min}$. The low values of the (002) FWHM suggested good crystal structure. However, as can be seen from Figure 4.26 the values of the FWHM increase with deposition rate, which may be indicative of some deterioration in the crystalline quality of films at the higher deposition rates. 


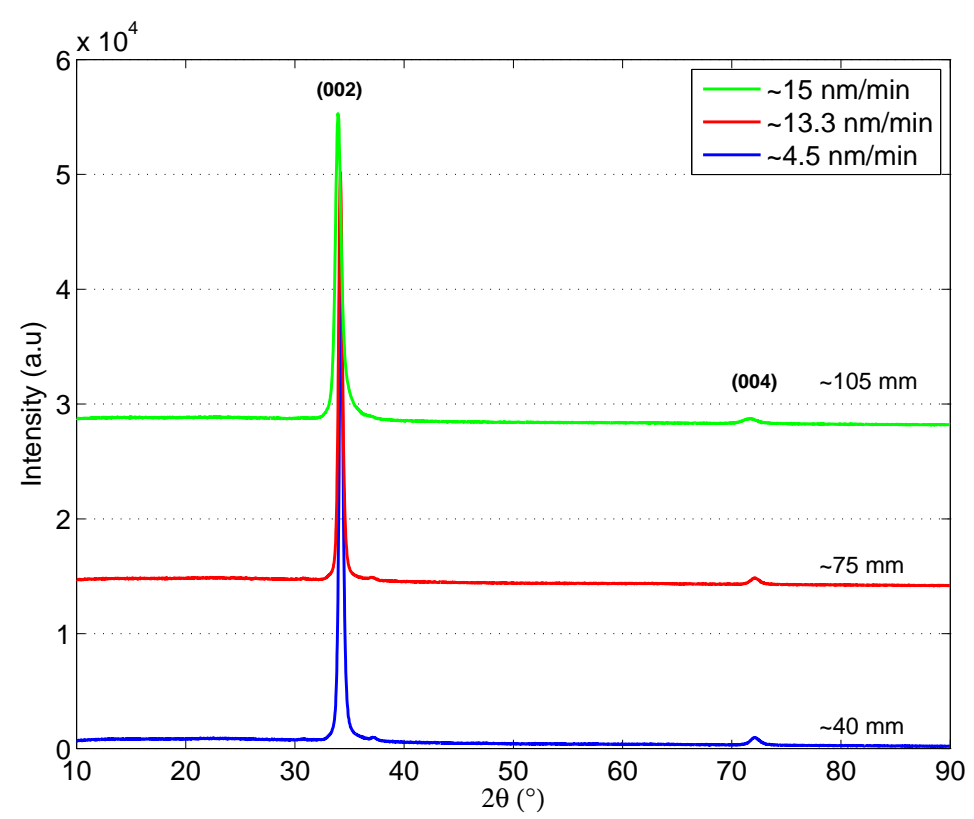

(a)

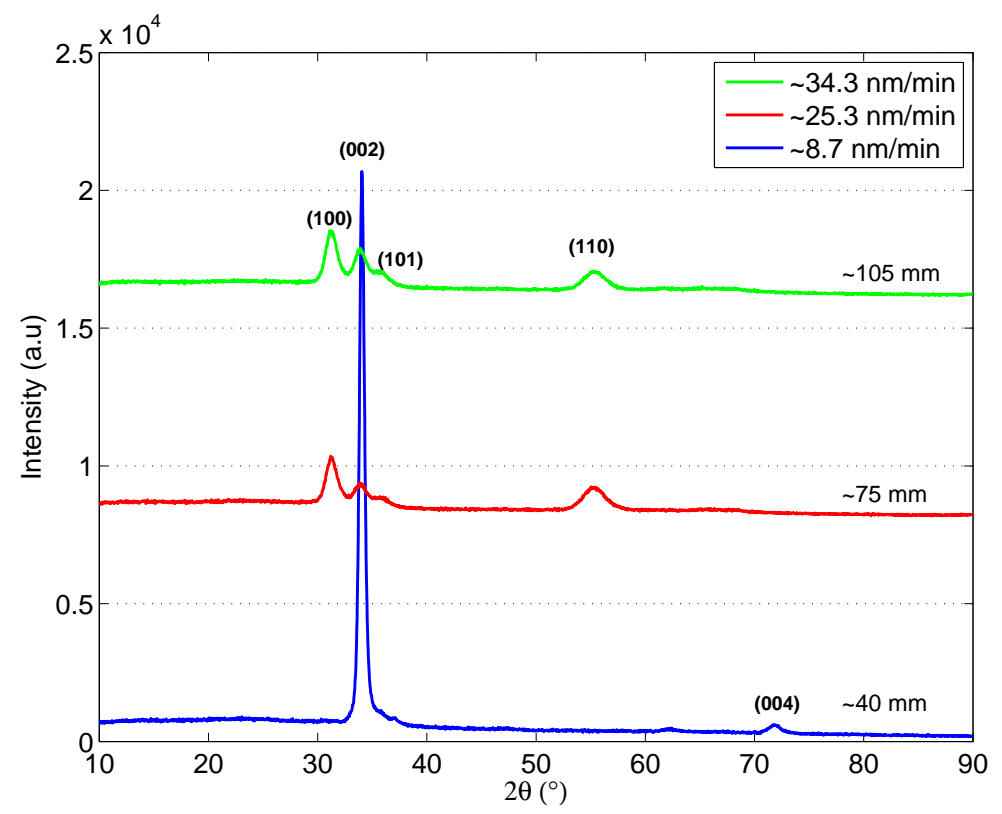

(b)

Figure 4.25: Influence of RF power on $\mathrm{ZnO}$ crystal quality at (a) $150 \mathrm{~W}$ and (b) $240 \mathrm{~W}$. All other parameters held constant: $\mathrm{Ar}=6 \mathrm{sccm}$, no external heating and sputtering time $=60$ mins . 


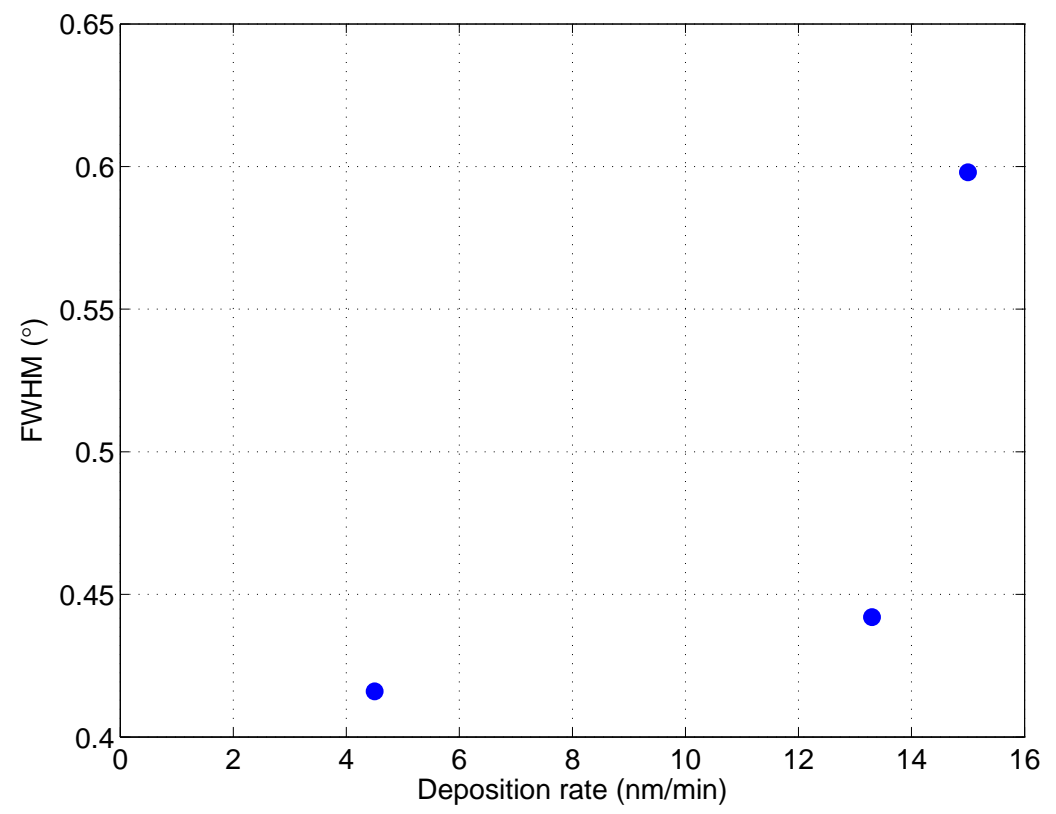

Figure 4.26: At an RF power of $150 \mathrm{~W}$ the FWHM increases with increasing deposition rate.

\subsubsection{Introduction of a buffer layer structure}

The improved crystal structure obtained from the deposition at $150 \mathrm{~W}$ suggested that the use of a two-step deposition process may help to maintain both c-axis alignment with good crystal structure, as well as high deposition rates in the films. Such a two-step process consisted of the following:

- Step 1: An initial nucleation step at low deposition rates that should form a well aligned c-axis film. This was typically done with an RF power of $150 \mathrm{~W}$.

- Step 2: The deposition rate can then be increased by an increasing the RF power (typically $240 \mathrm{~W}$ was used).

It was hoped that the initial slow deposition rate would produce a purely (001) textured film. This buffer layer would serve as the 'substrate' 
for further depositions and should help to reduce formation of unwanted orientations when the deposition rate is increased during the second step. It should be noted that such a two-step process will only be effective in reducing unwanted orientations if these orientations are the direct result of the amorphous substrate.

Figure 4.27 shows the obtained XRD spectra from the new two-step deposition process. The two-step deposition produced very high deposition rates reaching a maximum of $\approx 28.1 \mathrm{~nm} / \mathrm{min}$ whilst maintaining (001) orientation. There is some deterioration and broadening of the (002) peak, at the higher deposition rates suggesting less crystal alignment, however no extra orientations are present.

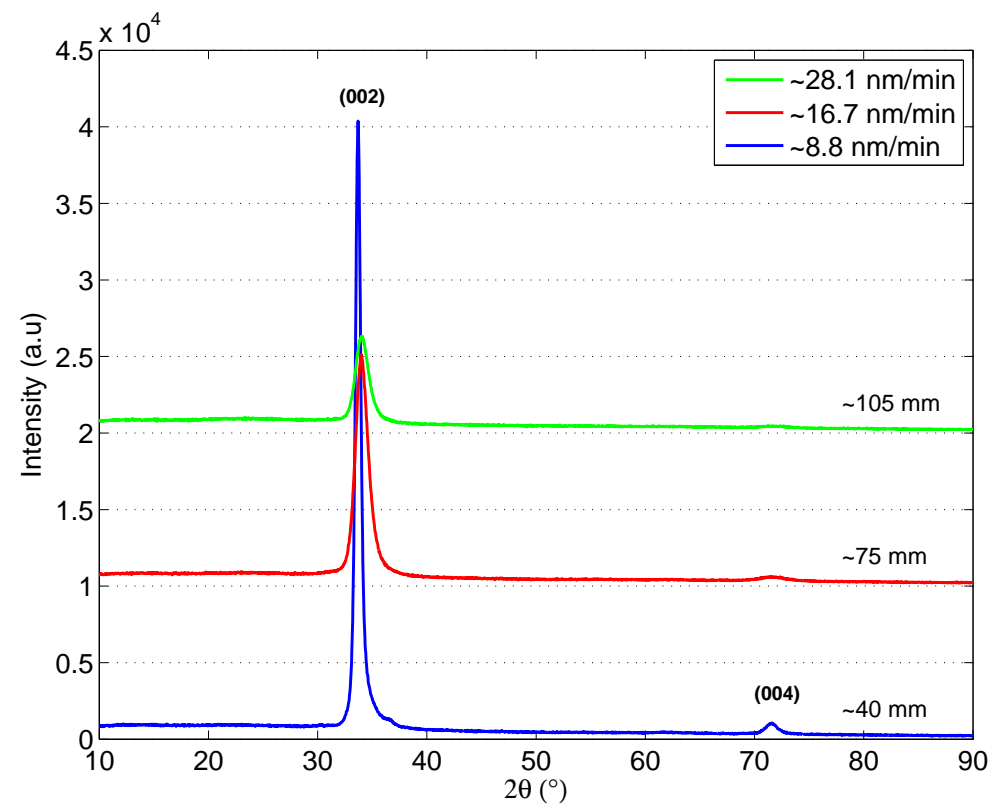

Figure 4.27: 2-step deposition: depositing a thin, (001) oriented $\mathrm{ZnO}$ layer to promote epitaxial growth for a faster deposited $\mathrm{ZnO}$ layer. All other parameters held constant: RF power $150 \mathrm{~W}$ for $20 \mathrm{~min}$ followed by $240 \mathrm{~W}$ for $60 \mathrm{~min}$ and $\mathrm{Ar}$ $=6 \mathrm{sccm}$.

With the introduction of a two-step deposition process good (001) orientation has been maintained at very high deposition rates, with a max- 
imum of $\approx 28.1 \mathrm{~nm} / \mathrm{min}$ being reached. The buffer layer facilitates the initial nucleation and encourages epitaxial layer growth for the faster deposited $\mathrm{ZnO}$ over-layer.

\subsubsection{Interdependence of film structure and surface mor- phology}

When the films were studied there was a clear correlation between the surface morphology and crystal structure as observed by the SEM images and as measured by XRD.

The surface morphology and cross section of an (001) oriented film is shown in Figure 4.28. The films surface exhibits a homogeneous surface structure, with rounded shaped grains of uniform size $(\approx 50-100 \mathrm{~nm})$ and well defined grain boundaries. The cross sectional image shows the strong columnar grain structure expected for an (001) oriented film.

Figure 4.29 shows the surface morphology and cross sectional image for a polycrystalline film. Compared to the (001) oriented film the surface is different. The grains are oblong facets with pointed edges, randomly grown in many directions. The random orientation of such facets can be attributed to the redistribution of existing molecules on the substrate surface as the result of competing planes within the film [102]. From the cross sectional image there appears to be no well defined texture.

The surface morphology and cross sectional image of a film deposited using the two-step deposition process, having a (001) orientation is shown in Figure 4.30. The surface morphology is similar to that of the (001) oriented film in Figure 4.28, except the grains appear larger in size with diameters ranging from $100-300 \mathrm{~nm}$. Similarly can be said about the cross sectional image, however, the columnar structure appears to be more well defined. The slow growth buffer layer was estimated to be $\approx 300 \mathrm{~nm}$ and is indicated on Figure $4.30 \mathrm{~b}$, it can be distinguished as the columnar structure is less defined. 


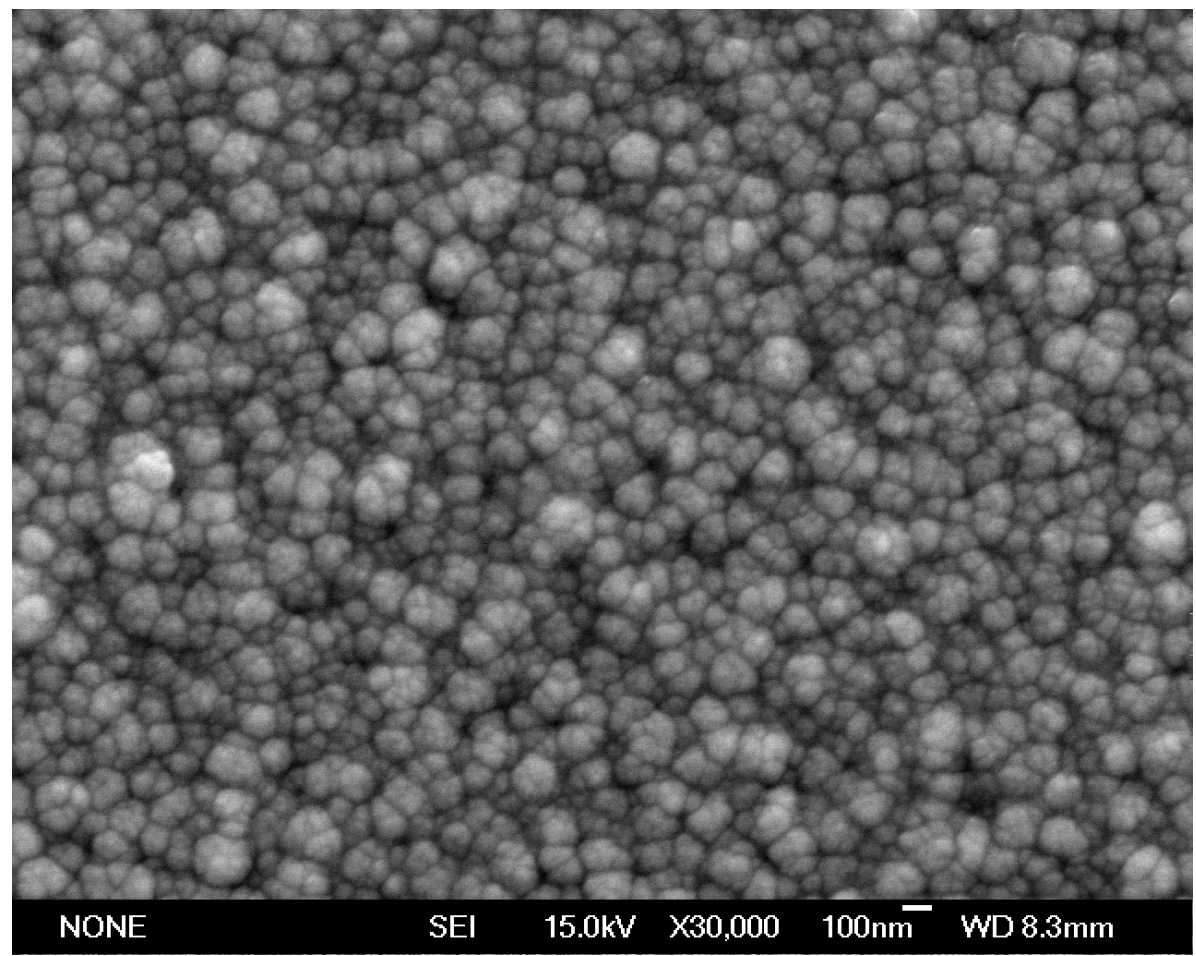

(a)

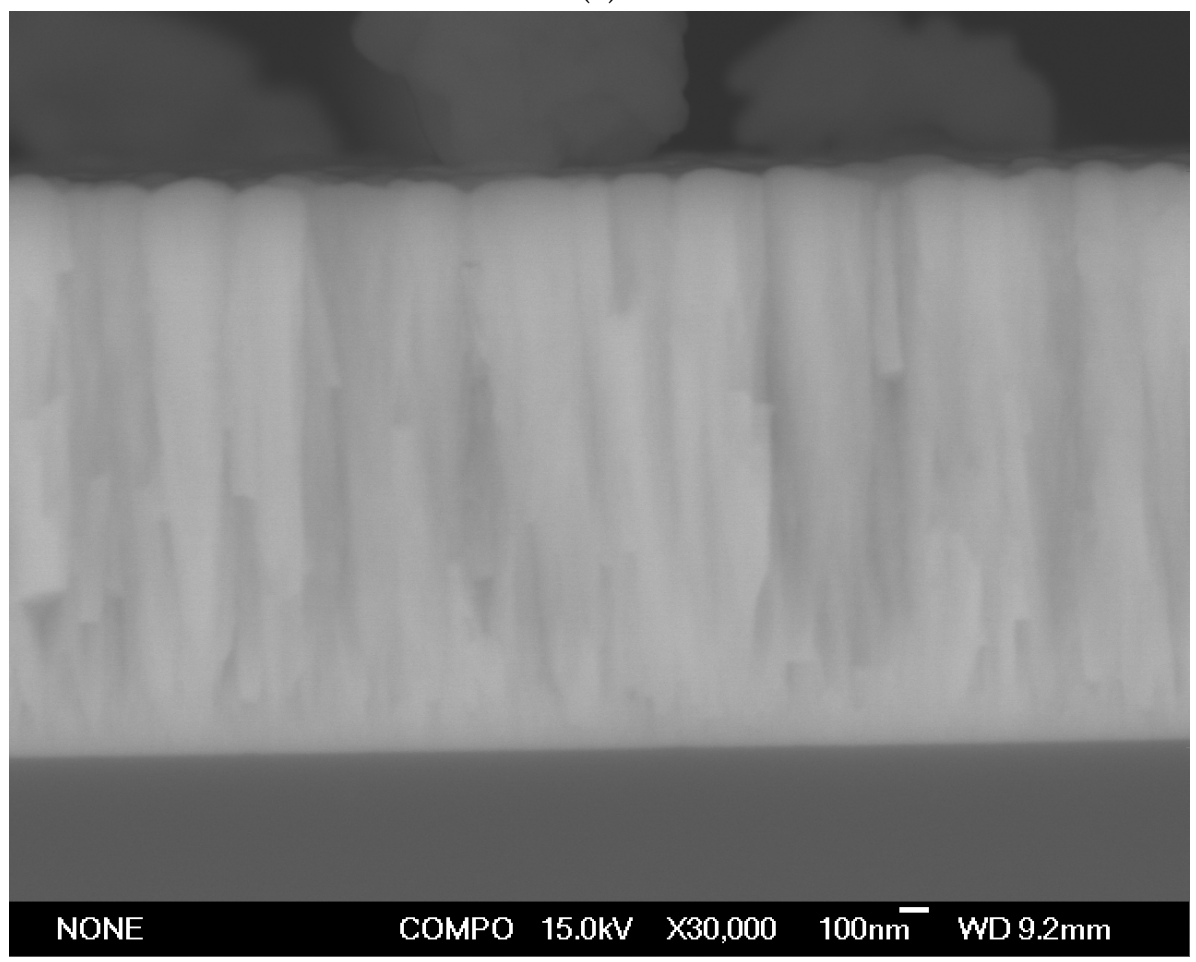

(b)

Figure 4.28: An (001) oriented film: (a) Surface morphology image, and (b) Cross sectional image. 


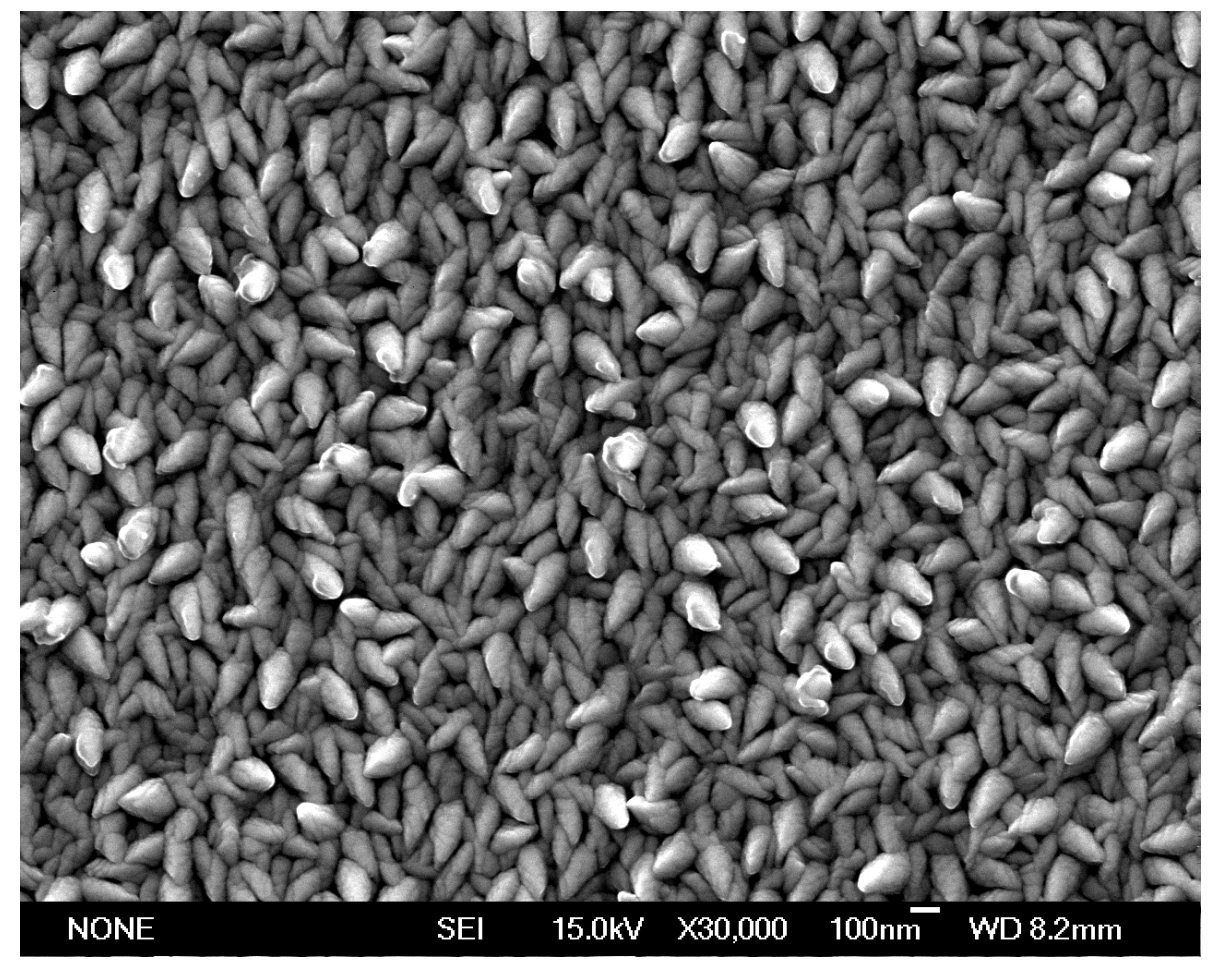

(a)

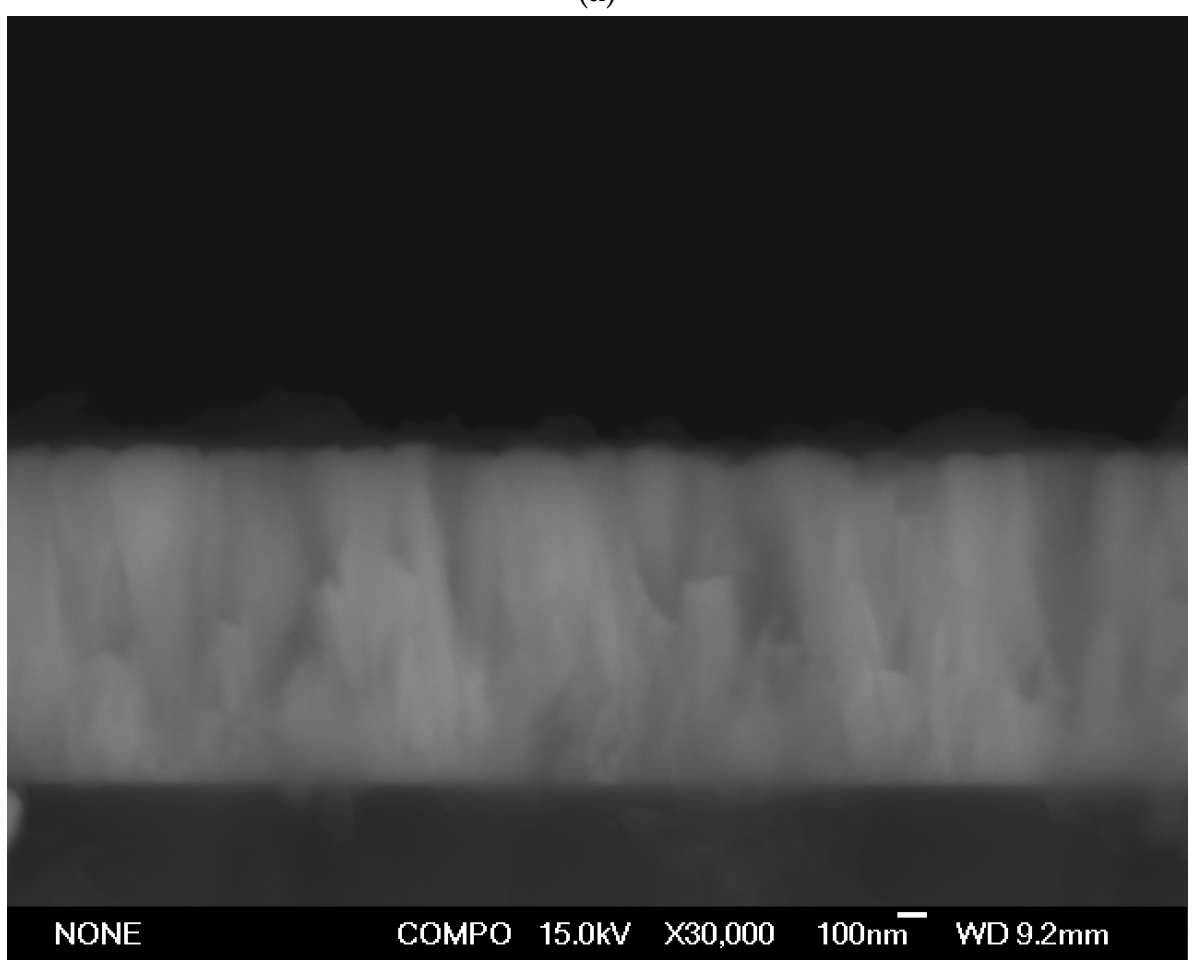

(b)

Figure 4.29: A polycrystalline film: (a) Surface morphology image, and (b) Cross sectional image. 


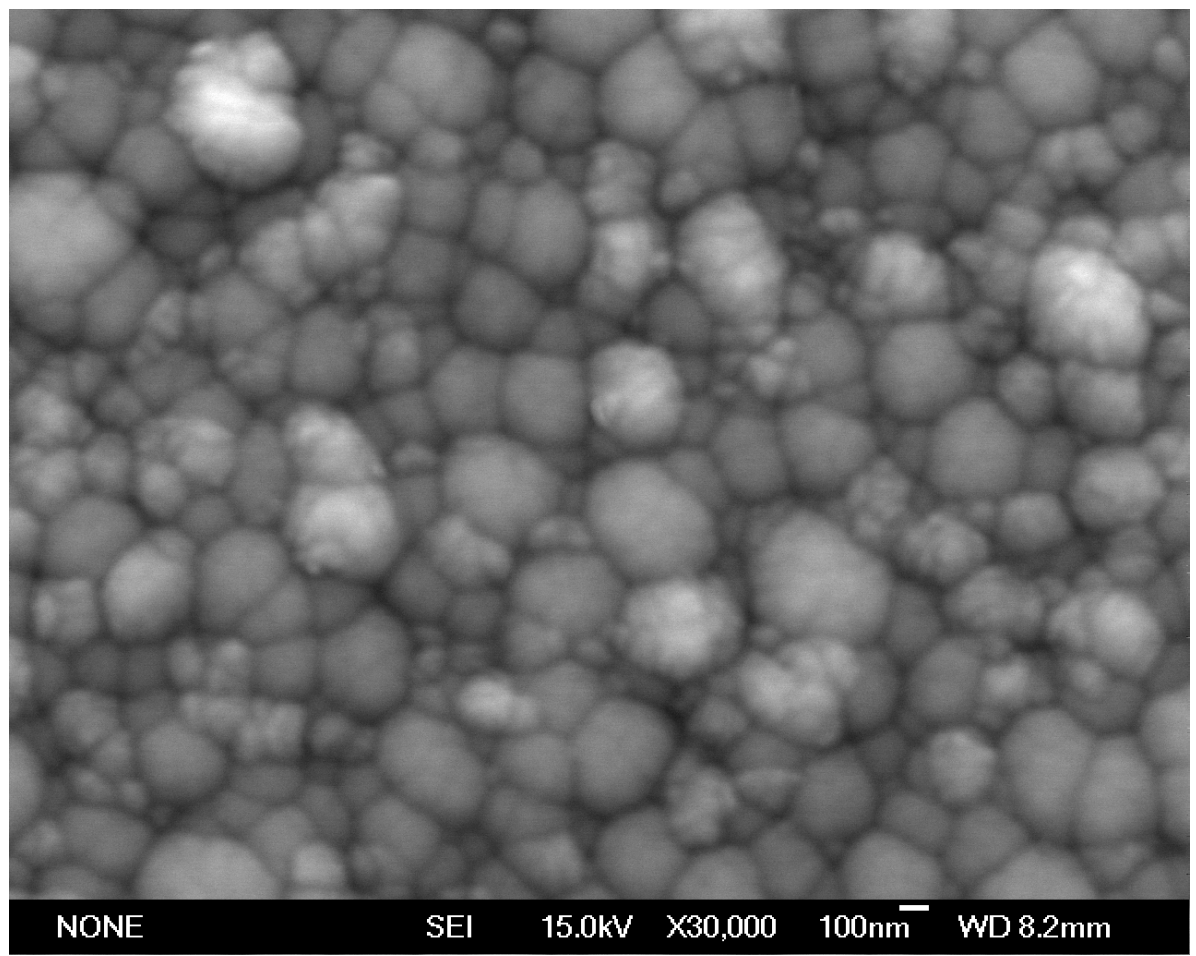

(a)

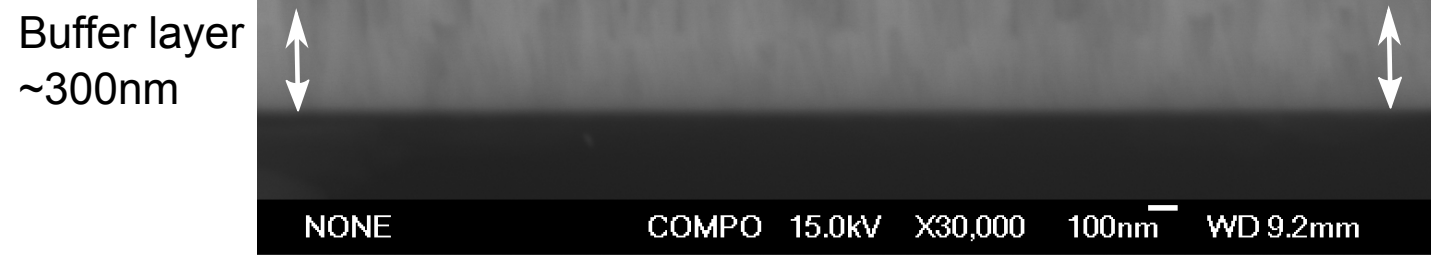

(b)

Figure 4.30: An (001) oriented film grown using the two-step deposition process (a) Surface morphology image, and (b) Cross sectional image. 
The texture of films with (001) orientation typically formed as those of Figures 4.28 and 4.30, having dense round grains. Whereas films deposited having a polycrystalline structure, as indicated by extra reflections present in the XRD spectra typically had texture similar to Figure 4.29, where the structure was less uniform and grains oblong in shape.

\subsubsection{Effects of the buffer layer structure with substrate heat- ing}

When investigating the influence of substrate temperature on film quality extra orientations were present. In an effort to suppress these extra orientations the two-step deposition process was investigated with substrate heating applied at $150^{\circ} \mathrm{C}$. The XRD spectra in Figure 4.31 compares this deposition to the two-step deposition performed with ambient substrate temperature. With the addition of the buffer layer, films grown at the primary deposition position were mostly dominated by extra orientations and were identified as the (100), (101), (110), (112) and (201).

From these results it was determined that films grown at ambient substrate temperatures produced the best quality (001) oriented $\mathrm{ZnO}$ films. 


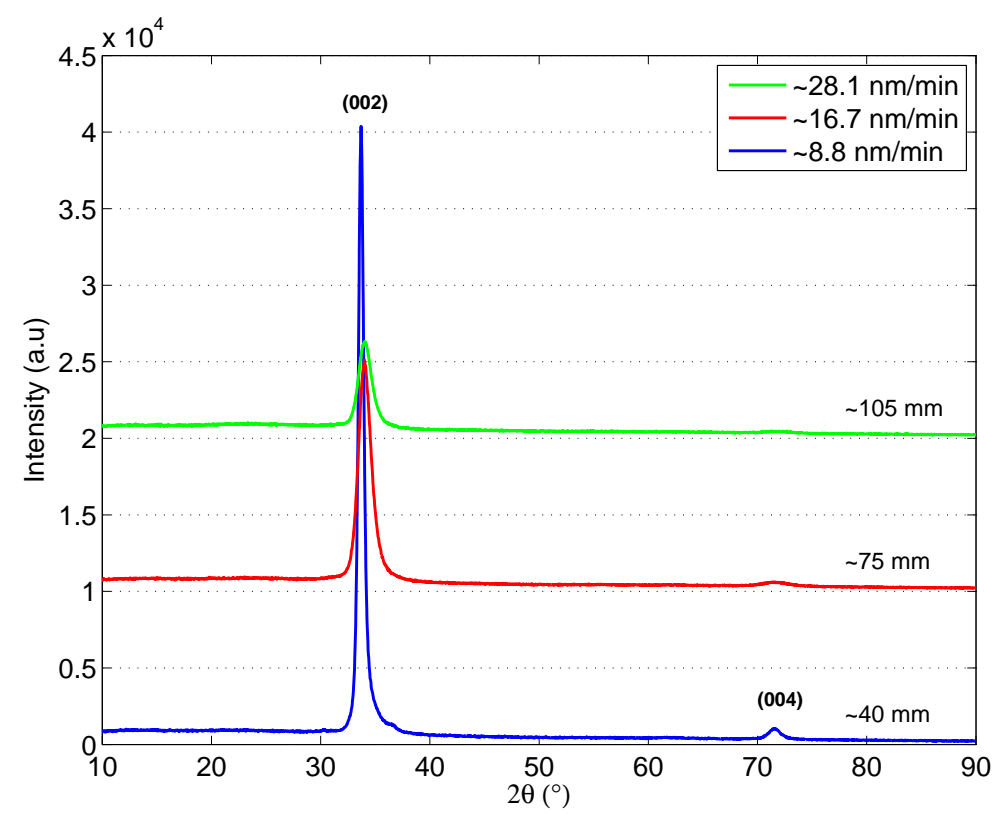

(a)

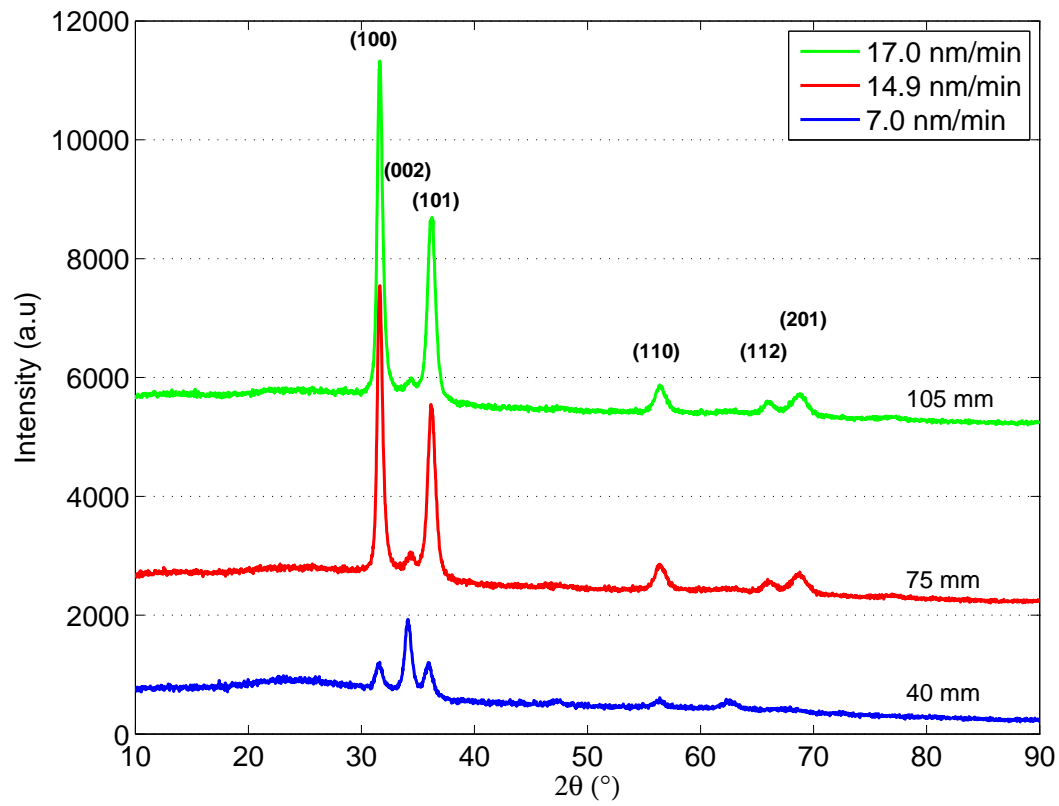

(b)

Figure 4.31: Influence of 2-step deposition on crystal quality with substrate temperatures at (a) Ambient, and (b) $150^{\circ} \mathrm{C}$. All other parameters held constant: RF power $150 \mathrm{~W}$ for 20 mins then $240 \mathrm{~W}$, Ar $=6 \mathrm{sccm}$ and sputter time $=60$ mins. 


\subsubsection{Conclusions form modification \#1}

The substrate-to-target distance was reduced in an effort to increase the deposition rate. The effects of substrate position, substrate heating and RF power were investigated at the new height. These results led to the following conclusions:

- Increased deposition rates were observed after the target-to-substrate distance had been reduced. By reducing this distance, the distance a sputtered atom must travel is decreased. As such, there is a smaller probability that the atom will collide with a surrounding particle before reaching the substrate.

- Film uniformity was maintained when depositing with a rotating holder, however the deposition rate was still too low. It was dramatically increased when depositing on a stationary holder, on substrates directly above the $\mathrm{ZnO}$ target. This led to a primary growing position where deposition rates up to $\approx 34 \mathrm{~nm} / \mathrm{min}$ were reached. However, only when depositing at deposition rates below $\approx 15 \mathrm{~nm} / \mathrm{min}$ were films preferentially (001) oriented. Depositing at higher deposition rates resulted in polycrystalline films. Similar results have been found by Molarius et al. [103] who suggested that the deposition rate should be kept at $\approx 16.67 \mathrm{~nm} / \mathrm{min}$ or lower to achieve high-quality ZnO films.

- Depositing with substrate heating at $150^{\circ} \mathrm{C}$ was investigated to see if (001) oriented films could be grown at the higher deposition rates (reaching $\approx 30 \mathrm{~nm} / \mathrm{min}$ ). The crystal quality was found to be very poor with the films being dominated by extra orientations.

- To preserve crystal quality at the central substrate position the deposition rate was reduced by decreasing the applied RF power to $150 \mathrm{~W}$. Although (001) orientation was maintained, a maximum de- 
position rate of $\approx 15 \mathrm{~nm} / \mathrm{min}$ was reached, which was still less than the minimum rate required.

- Due to the good crystal quality attained when depositing at $150 \mathrm{~W}$ RF power and the high deposition rates achieved at $240 \mathrm{~W}$ the two were combined to develop a two-step deposition process. The first step consisted of a slow grown buffer layer which promoted epitaxial growth for a second layer deposited at a much faster rate. This new process allowed deposition rates of up to $\approx 28 \mathrm{~nm} / \mathrm{min}$ whilst maintaining good crystal quality.

- Finally, substrate temperature was re-examined using the new twostep process, however the films still contained mixed orientations.

The finding that an increase in substrate temperature leads to a deterioration in the c-axis orientation of the films is a surprising result, and appears to be contrary to many reports on $\mathrm{ZnO}$ sputtering in the literature. However, alternative conclusions have also been found. Studies by Singh and Cho et al. $[84,94]$ found that increased substrate temperature improves $\mathrm{ZnO}$ (001) quality, arguing that when depositing at ambient temperature, atoms condensing on the substrate surface will not have enough kinetic energy to reach the position of their lowest surface energy, forming (001) orientation. And that an increased substrate temperature provides the atoms with energy allowing them to move to more stable sites, ultimately forming (001) orientation. However, other studies found that at higher substrate temperatures extra orientations become present, which did not occur when depositing at ambient temperature [102,104]. Theoretically, increased substrate temperature would increase the thermal stress between the film and substrate surface, therefore it seems practical to deposit at lower substrate temperatures to reduce thermal stress. 


\subsection{Origin of film cracking and delamination}

The previous work on the development of a two-step deposition process provided the basis for a viable process for the deposition of c-axis oriented $\mathrm{ZnO}$ films for piezoelectric applications. Relatively high growth rates up to $\approx 28 \mathrm{~nm} / \mathrm{min}$ could be achieved which would allow the deposition of $3 \mu \mathrm{m}$ thick films in $\approx 1.8$ hours. Increased deposition times were now used to grow films to a thickness suitable for device processing. However, it was noticed that many of the thicker films $(>1.5 \mu \mathrm{m})$ showed signs of cracking and in more extreme cases delaminated from the substrate surface. This phenomenon appeared to be most severe on the thicker regions of the film and most often occurred on the part of the substrate directly above the $\mathrm{ZnO}$ target.

The observed cracking and delamination process of the films can be seen in Figure 4.32. On some films which had not experienced peeling, crack generation was still observed, as can be seen from Figure 4.32a. These cracks would progressively increase in size $4.32 \mathrm{~b}$, after which, portions of the film become completely removed exposing the bare glass substrate 4.32c. An interesting observation can be seen in Figure 4.32d where a photograph shows a circular cloudy region across the $\mathrm{ZnO}$ films where on the bottom right side shows the early signs of cracking and peeling. The circular region had been observed on several of the films, and was suspected to be the influence of the magnetron on the plasma, as the diameter of the ring was the same as the racetrack erosion on the $\mathrm{ZnO}$ sputter target. 


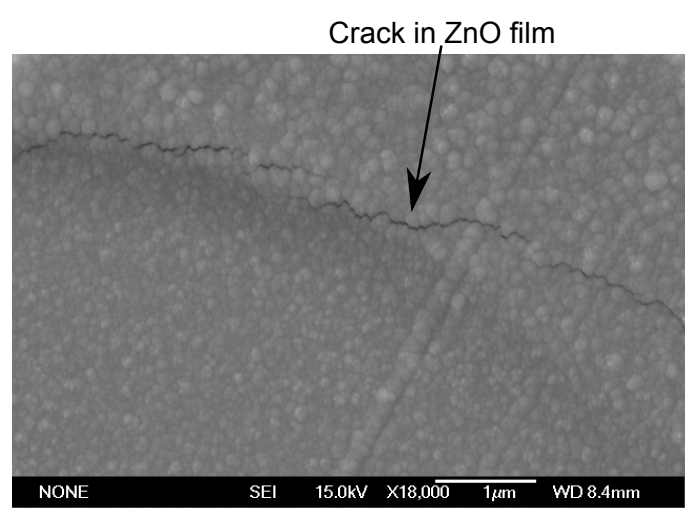

(a)

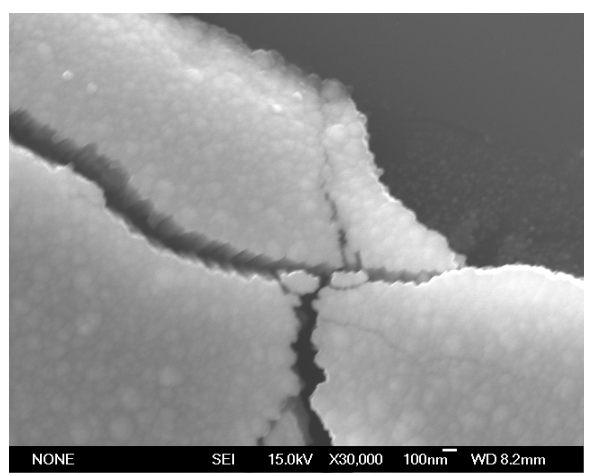

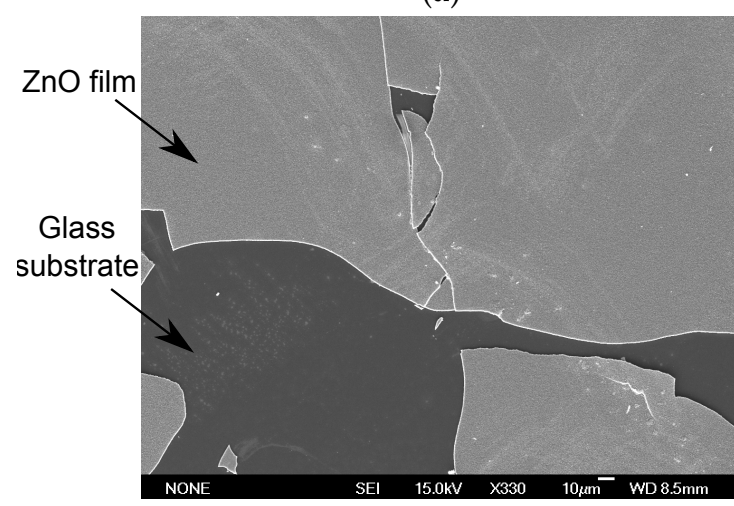

(c) (b)

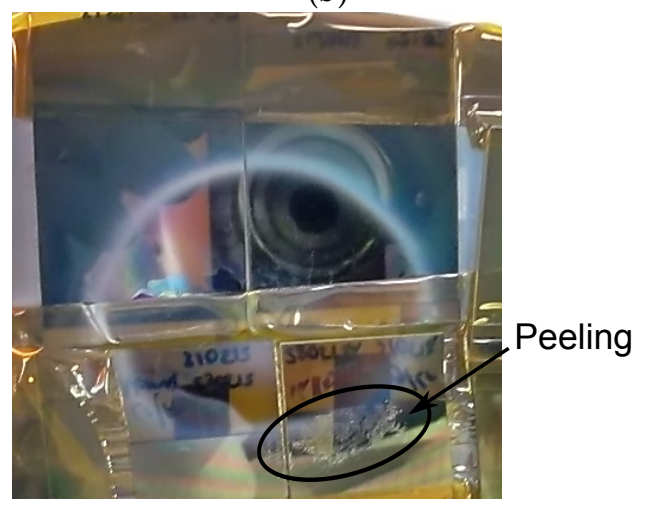

(d)

Figure 4.32: Images of cracked $\mathrm{ZnO}$ films: (a) An SEM image of a crack through a ZnO film, (b) An SEM image of a progressively larger crack, (c) An SEM image of a cracked film which has also peeled in some regions, (d) The initial cracking and peeling on the layer, observed on the region of the ring. The dark image in the photo is the reflection of the camera.

The samples were also imaged cross sectional in the SEM showing the delamination of the film from the substrate surface. As can be seen in Figure 4.33a, the film appears to have buckled. In extreme cases, the film became completely delaminated from the substrate, where Figure $4.33 \mathrm{~b}$ shows a $\mathrm{ZnO}$ flake resting on the substrate surface. The direction of the bowing indicates that the film may have been under a large amount of compressional stress. 


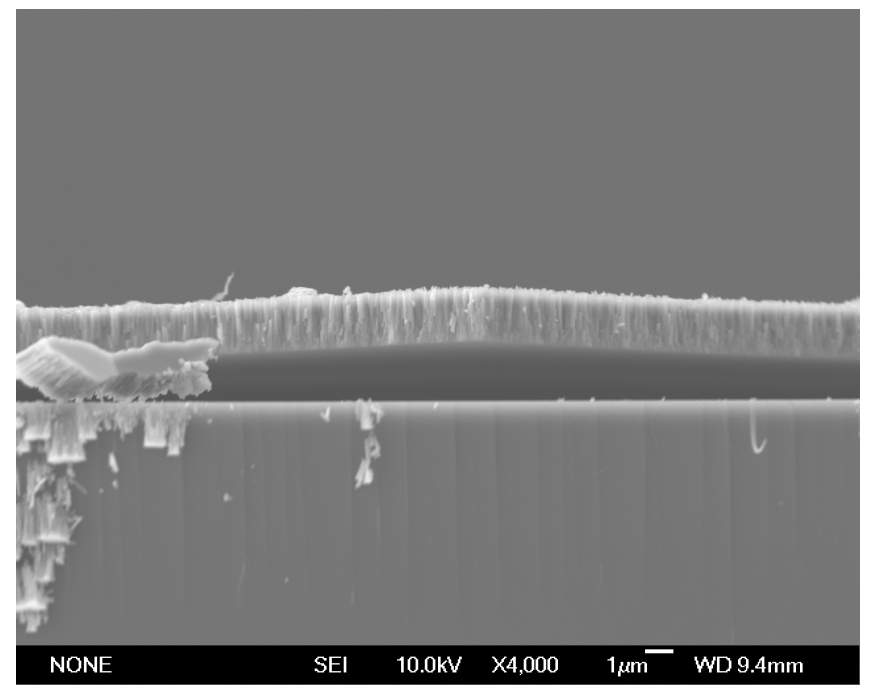

(a)

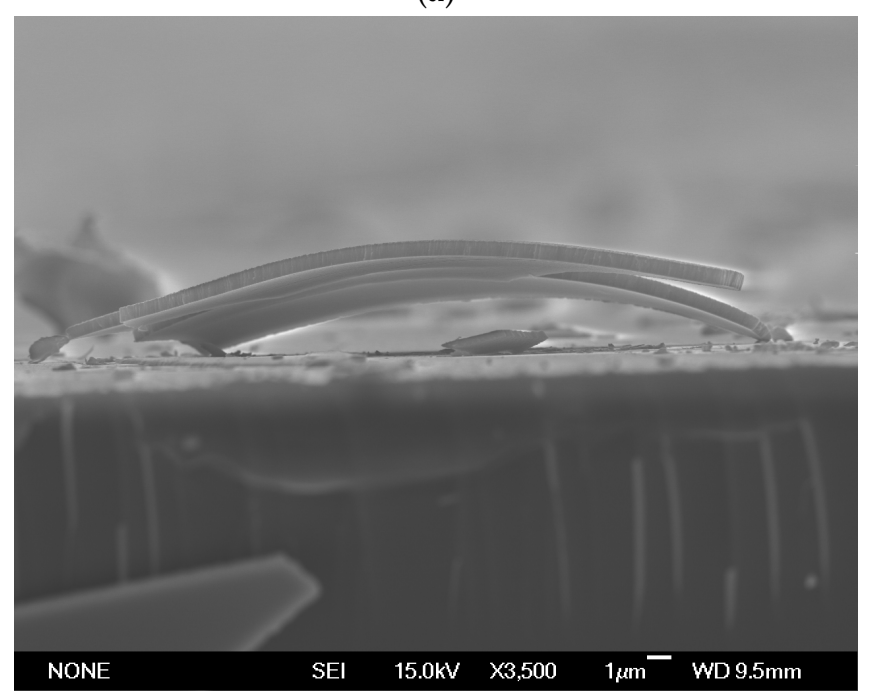

(b)

Figure 4.33: Cross sectional images of delaminated $\mathrm{ZnO}$ films: (a) $\mathrm{ZnO}$ film peeling from the substrate surface, and (b) A flake of $\mathrm{ZnO}$ resting on the substrate surface. The bowing direction of these films indicates that they were compressively stressed.

The cracking and delamination of the $\mathrm{ZnO}$ films made them unsuitable for any device processing and it was essential for this problem to be solved 
before any further development work could be undertaken. There were two potential causes considered for these cracked and delaminating films:

- Poor substrate-to-film adhesion

- Stress in the $\mathrm{ZnO}$ films

These two potential causes will now be considered in more detail.

\subsubsection{Poor substrate-film adhesion}

Any organic residues or other contaminants left on the substrate would cause poor substrate-film adhesion and potentially lead to the deposited film peeling from the substrate. For this reason several different cleaning procedures were employed. These cleaned substrates were also compared to the as-received substrates from the manufacturer which had not been cleaned prior to the deposition. However, none of the film depositions onto substrates prepared using the alternative cleaning procedures investigated showed any noticeable difference to films which had been deposited using our standard procedure.

In order to investigate substrate-film adhesion the scotch-tape test was performed. This consisted of sticking a piece of sellotape on a thin, noncracking film and then peeling it off. All films proved very robust, and no peeling was initiated when the sellotape was pulled off. And indicated that for thinner films the adhesion between the substrate and film was very good. This suggested that poor substrate film adhesion was not the primary reason for the cracking and delamination of the $\mathrm{ZnO}$ films. However, it could still be a contributing factor.

\subsubsection{Stress in $\mathrm{ZnO}$ film}

There are several factors which could contribute to stress within thin films. As was discussed in Section 2.3.4 film stress can be classified as intrinsic or extrinsic. Intrinsic stress is induced during the deposition process, 
whereas extrinsic stress is induced by external factors, including lattice mismatch and thermal stresses. The total stress experienced by the film is the addition of both intrinsic and extrinsic stresses. The origins of different types of stress and their possible contribution to stress in our $\mathrm{ZnO}$ films are discussed below.

\section{Lattice mismatch between substrate and film:}

Mismatch in the lattice parameters between the substrate and film is a well known source of stress in thin films. This occurs when the lattice of the depositing film may be elastically strained to conform to the lattice parameters of the substrate. The strain in the film is typically relaxed at a critical thickness by the introduction of misfit dislocations into the film [105]. However, as depositions were performed on amorphous glass substrates, having no regular lattice parameters, this mechanism was not considered to be the primary reason for the introduction of stress within the films.

\section{Thermal mismatch between substrate and film:}

The mismatch in the coefficient of thermal expansion (CTE) between a substrate and film can induce stress in the film when it is cooled from the deposition temperature to room temperature. During a deposition, the film and substrate expand as the temperature in the chamber increases. After the deposition, the film and substrate begin to cool down; they shrink by different amounts, depending on their CTE. The problem then arises when one shrinks more than the other which induces stress within the film. If the stress becomes too large, it is likely the film will release it through cracking and potentially delaminating from the substrate surface.

The CTE for $\mathrm{ZnO}$ and soda-lime glass is shown in Table 4.1. The thermal stress, $\sigma_{T h}$, can be calculated from [106],

$$
\sigma_{T h}=\frac{E_{f}}{1-v_{f}}\left(\alpha_{s}-\alpha_{f}\right)\left(T-T_{d}\right)
$$


where $E_{f}$ is the Youngś modulus of the film, $v_{f}$ is the Poisson ratio of the film and $\alpha_{s}$ and $\alpha_{f}$ are the CTE of the substrate and film respectively. The temperature difference between room temperature and the deposition temperature is given by $T-T_{d}$.

Poisson's ratio is given by [21],

$$
v_{f}=\frac{C_{13}}{C_{11}+C_{12}}
$$

where $C_{11}, C_{12}$ and $C_{13}$ are 208.8 $G P a, 119.7 G P a$ and 104.2 GPa (taken from Table 3.2).

$E_{f}$ is the Youngś modulus and is calculated from [21],

$$
E_{f}=3 B\left(1-2 v_{f}\right)
$$

where $v_{f}$ is Poisson's ratio and $B$ the bulk is modulus (160 GPa as used by [21]).

Table 4.1 shows the calculated thermal stress present in a $\mathrm{ZnO}$ film when deposited onto a soda-lime substrate at different temperatures. Because these are negative stress values, it would indicate that a compressive stress would be observed in the $\mathrm{ZnO}$ film.

\begin{tabular}{|c|c|c|c|c|c|c|}
\hline Substrate & $\begin{array}{c}\mathrm{CTE} \text { at } 25^{\circ} K \\
\times 10^{-6} K^{-1}\end{array}$ & $\begin{array}{c}\sigma_{T h} \text { at } 50^{\circ} K \\
(\mathrm{GPa})\end{array}$ & $\begin{array}{c}\sigma_{T h} \text { at } 100^{\circ} \mathrm{G} \\
(\mathrm{GPa})\end{array}$ & $\begin{array}{c}\sigma_{T h} \text { at } 150^{\circ} K \\
(\mathrm{GPa})\end{array}$ & $\begin{array}{c}\sigma_{T h} \text { at } 200^{\circ} K \\
(\mathrm{GPa})\end{array}$ & $\begin{array}{c}\sigma_{T h} \text { at } 250^{\circ} K \\
(\mathrm{GPa})\end{array}$ \\
\hline $\begin{array}{c}\text { Soda lime } \\
\text { glass }\end{array}$ & 9.35 & -0.055 & -0.110 & -0.166 & -0.221 & -0.276 \\
\hline $\begin{array}{c}\text { Borosilicate } \\
\text { (Pyrex 7740) }\end{array}$ & 3.25 & 0.0180 & 0.036 & 0.0540 & 0.072 & 0.090 \\
\hline ZnO film & 4.75 & & & & & \\
\hline
\end{tabular}

Table 4.1: Thermal stress induced by soda lime glass and borosilicate at various substrate temperatures. CTE obtained for $\mathrm{ZnO}$ [58], soda-lime glass [107] and borosilicate [89].

Due to the fact that many of the depositions were performed under ambient temperatures $\left(\approx 50^{\circ} \mathrm{C}\right)$, the contribution of thermal stress is likely 
to be relatively low. However, in order to reduce thermal mismatch as the source of the stress, it was decided to perform depositions on substrates which had a better thermal match with $\mathrm{ZnO}$. Borosilicate glass is a strong candidate with a CTE of $3.25 \times^{-6} K^{-1}$ [89], relatively close to that of $\mathrm{ZnO}$ at $4.75 \times{ }^{-6} K^{-1}$. Table 4.1 also shows the expected contribution of thermal stress with films deposited onto borosilicate substrates at different temperatures. The positive stress values indicate the film would be under tensile stress. Also, as would be expected, the contribution of thermal stress from depositing on the borosilicate is less than when depositing onto soda-lime glass.

A series of depositions were performed on borosilicate substrates obtained from two different suppliers. However, no significant difference in the cracking and peeling behaviour of the films was found, compared to those deposited on the soda-lime glass substrates. Although thermal mismatch between the $\mathrm{ZnO}$ film and glass substrate could contribute to the overall stress, it was concluded that this was not the dominant source.

\section{Intrinsic stress due to the deposition process:}

It is often described that physical deposition processes such as thermal evaporation, e-beam evaporation or sputtering may lead to high levels of stress in the resultant films [70,108-110]. This is mainly ascribed to the process of atomic peening, where the film is constantly bombarded by energetic particles from the vapour phase [111]. This can be considered analogous to the metallurgical peening process, where a metal is constantly hit with a small hammer in order to produce a densification of the metal structure. In the case of physical deposition processes, the bombardment of the film by energetic particles will also produce a densification of the layer, leading to compressive stress in the films.

The intrinsic stress formation during the sputtering process was eventually considered to be the main mechanism for stress introduction; eventually leading to the mechanical failure of the films. It was essential for 
this mechanical failure to be avoided in order to yield material for device processing. We thus tried to systematically study the observed stress in the films and correlate this stress to any of the deposition parameters.

\subsubsection{Influence of deposition parameters on stress}

The stress within films was calculated using the XRD spectra as described in Section 3.4.3. It was estimated by calculating the strain along the c-axis as seen by a shift in the $2 \theta$ peak position of the (002) reflection, compared to the unstrained value of bulk $\mathrm{ZnO}$, using Equation 3.9. This strain was then related back to stress through Hooke's law (Equation 3.7). The stress was classified as either compressive, indicated by a negative stress value or tensile, as indicated by a positive value. Because the stress was calculated from the $2 \theta$ peak position, stress at the exact point of peeling could not accurately and reliably be determined. However, because there were always three substrates present during the deposition (see Figure 4.20 for sample configuration) and not all areas of the substrates showed peeling, the overall stress inherent to the deposition process could be analysed. The stress induced by the sputtering parameters RF power and deposition pressure was evaluated.

\subsubsection{Effect of RF power}

As the occurrence of intrinsic stress was thought to be associated with the bombardment of the film with energetic particles, the first parameter investigated was the RF power. Films had been deposited at powers of $150 \mathrm{~W}, 240 \mathrm{~W}$ and 150/240 $\mathrm{W}$ using the two-step deposition process. Irrespective of RF power, some peeling occurred on the central substrate, while the other two films remained intact. The calculated stress from the intact films was always compressive and found to be in the range of $-0.41 G P a$ to $-4.87 G P a$. From these observed stress values, it became obvious that the level of compressive stress increased with increasing de- 
position rate, shown in Figure 4.34 for the various RF power depositions. A value of $\approx-4 G P a$ can also be estimated as the yield of stress, as this was typically the maximum stress value observed on an intact film. (The point of -4.9 GPa in Figure 4.34c appears to be an outlier).

The influence of RF power on the observed stress was evaluated by comparing the stress at the same deposition rate across each of the depositions. A rate of $\approx 10 \mathrm{~nm} / \mathrm{min}$ was selected as films grown at this rate were known to have good (001) orientation. The results are shown in Table 4.2.

\begin{tabular}{|c|c|}
\hline $\begin{array}{c}\text { RF Power } \\
(\mathrm{W})\end{array}$ & $\begin{array}{c}\text { Est. Stress at } \\
10 \mathrm{~nm} / \min (\mathrm{GPa})\end{array}$ \\
\hline 150 & -1.9 \\
\hline 240 & -1.6 \\
\hline $150 / 240$ & -2.1 \\
\hline $150 / 240$ & -1.5 \\
\hline
\end{tabular}

Table 4.2: The estimated stress value at $\approx 10 \mathrm{~nm} / \mathrm{min}$ for the various RF powers.

These results were found to be inconclusive as there was no clear trend that RF power was the dominant factor contributing to the stress. 


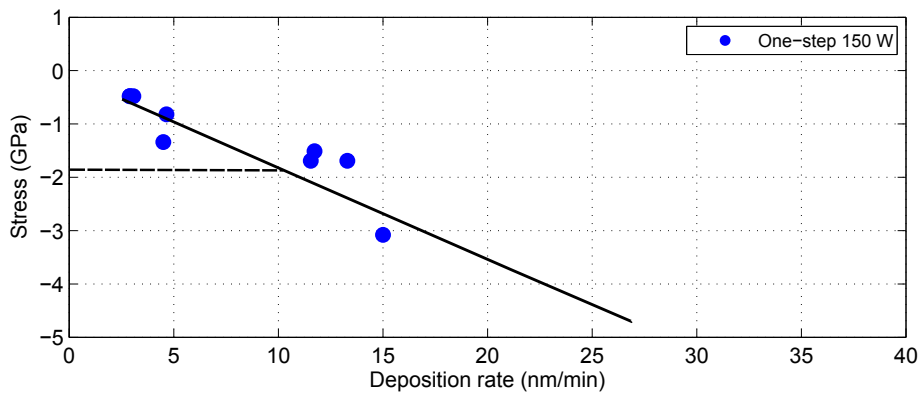

(a)

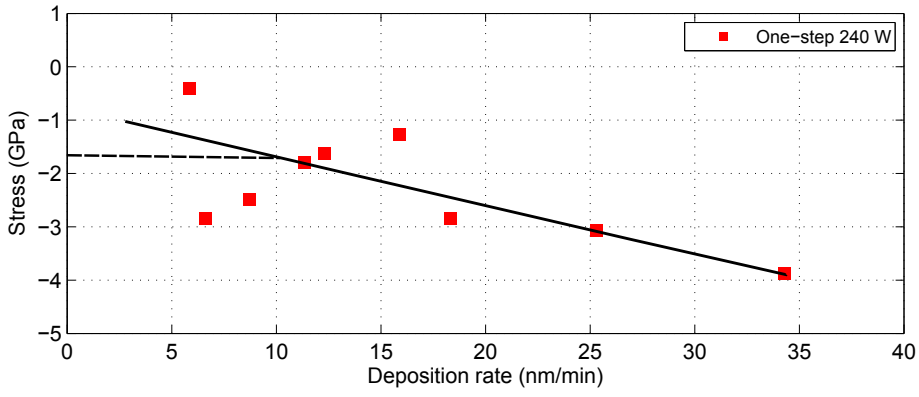

(b)

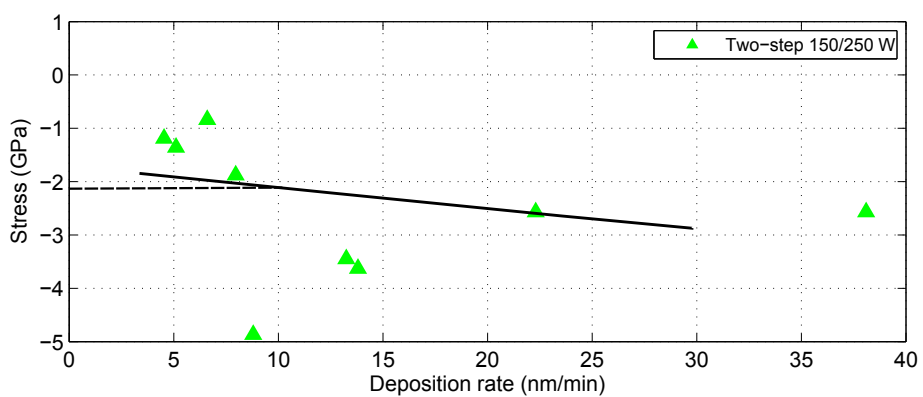

(c)

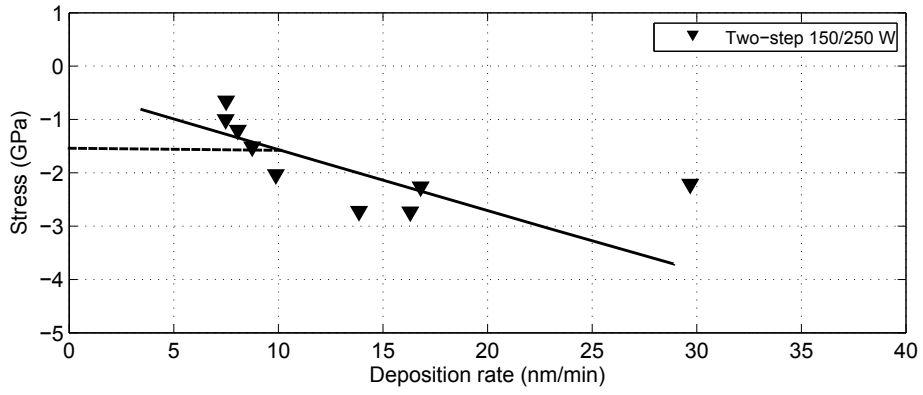

(d)

Figure 4.34: Calculated stress within the films as a function of deposition rate at various RF powers. (a) $150 \mathrm{~W}$, (b) $240 \mathrm{~W}$, (c) and (d) Two-step depositions $150 \mathrm{~W}$ for $20 \mathrm{~min}$ followed by $240 \mathrm{~W}$ for $60 \mathrm{~min}$. The dotted line indicates the observed stress at $10 \mathrm{~nm} / \mathrm{min}$. 


\subsubsection{Effect of deposition pressure}

While investigating the effect of RF power on the resultant stress it was noted that there also seemed to be a trend between the observed stress and chamber pressure during the deposition. As was discussed previously in Section 4.1.1, the setting of the chamber pressure was the combination of the gas flow into the chamber and the main valve position. As was seen from Figure 4.1, for a specific Ar flow there was a variation in the chamber pressure due to the main value position. Although this was regularly adjusted (so that at $\mathrm{Ar}=6 \mathrm{sccm}$ deposition pressure was $\approx 1.0 \times 10^{-2} \mathrm{mbar}$ ) there was day-to-day drift, and a result depositions were often performed slightly above and slightly below this chamber pressure.

Historical data was then investigated and depositions where films had peeled and had not peeled were compared to the deposition pressure. For each deposition where peeling had occurred, a critical thickness was defined. The critical thickness was the thickest region on that film where peeling had not occurred. Likewise for each deposition where films had not peeled, the thickest region of the film was defined as the critical thickness. The results from this investigation are shown in Figure 4.35. The points marked in blue indicate films which had peeled and points in red, films which had not peeled. It is clear from this graph that there seems to be a consistent trend in the critical thickness with deposition pressure. As the pressure is increased the critical thickness (estimated thickness below which the film was intact) also increased. For low deposition pressures $\left(\approx 8 \times 10^{-3}\right.$ mbar $)$ films peeled at $\approx 0.5 \mu \mathrm{m}$, this occurred only at $\approx 1.5 \mu \mathrm{m}$ when deposition pressure was $\approx 11 \times 10^{-3}$ mbar. Even more significantly, no peeling was observed in films deposited at pressure $>12 \times 10^{-3}$ mbar . 


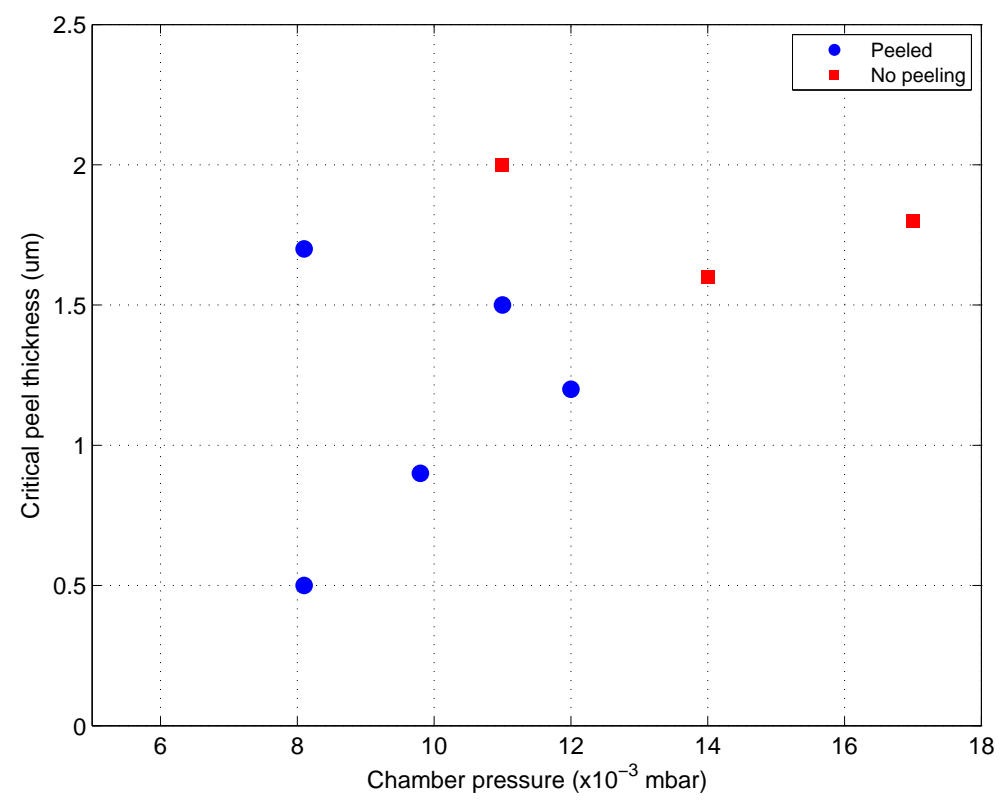

Figure 4.35: For various depositions, the graph shows the thickest regions on the films which had not peeled. This suggests that films deposited above $\approx 1.2 \times 10^{-2}$ did not peel.

This relationship between depositing at lower pressures and films peeling appeared to be the only correlating trend between the deposition parameters and film stress. Studies done by Windischmann, Jou and Cebulla et al. $[110,112,113]$ found similar results, suggesting that films became less compressively stressed when depositing at higher chamber pressures.

As a result, a 'critical pressure' of $1.2 \times 10^{-2}$ mbar was defined and it was proposed that all subsequent depositions be performed above this pressure in an effort to reduce the intrinsic stress within the films and consequently solve the peeling problem.

It was obvious that films deposited at higher pressures were still under some compressive stress. Provided films could now be deposited without cracking or peeling from the substrate surface, post growth annealing could be investigated as a reliable technique to relax the film stress. 


\subsubsection{Stress relaxation through post growth annealing}

During the above analysis it became obvious that all films were under large amounts of compressive stress. A popular technique used to relieve stress is through post growth annealing. It is also well known to improve crystalline structure $[71,82,100,114-116]$. The influence of post growth annealing on stress within some selected films was investigated. As a first quick test, films were placed on a hot plate at a temperature of $400^{\circ} \mathrm{C}$ and left for 1 hour in air.

The XRD spectra were measured before and after annealing so a comparison could be made. Figure 4.36 shows the annealing results of different films which had been annealed. In Figure 4.36a an (001) oriented film was annealed. The shift in the $2 \theta$ peak position can be attributed to a relaxation of stress within the film. The increase in intensity and reduction in the FWHM also suggests an improvement in crystalline structure. In Figure $4.36 \mathrm{~b}$ a polycrystalline film had been annealed. Annealing the film did not promote preferential growth. Instead, all orientations present increased in peak intensity, with the (100) and (101) increasing the most. The FWHM also decreased for each of the orientations. Fujimura et al. [79] found this same result concluding that films should be (001) orientated prior to the annealing process. From the first experiment it was clear that for (001) orientated films, stress could be released through post deposition annealing. 


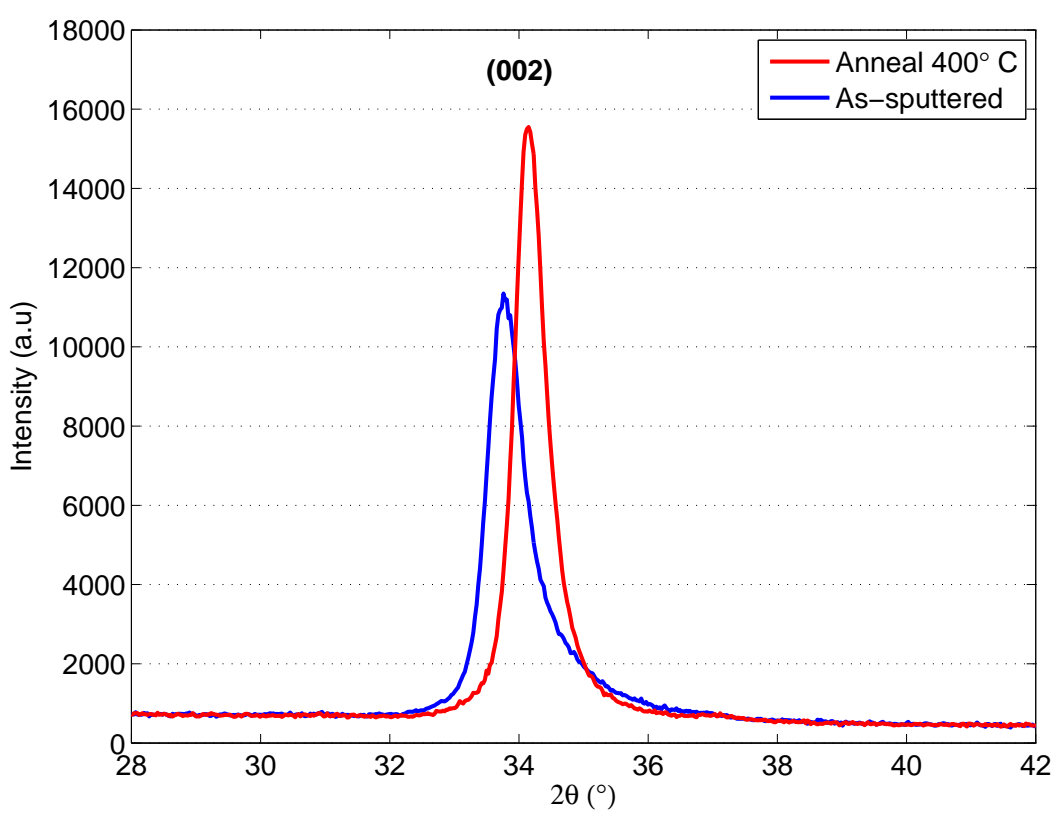

(a)

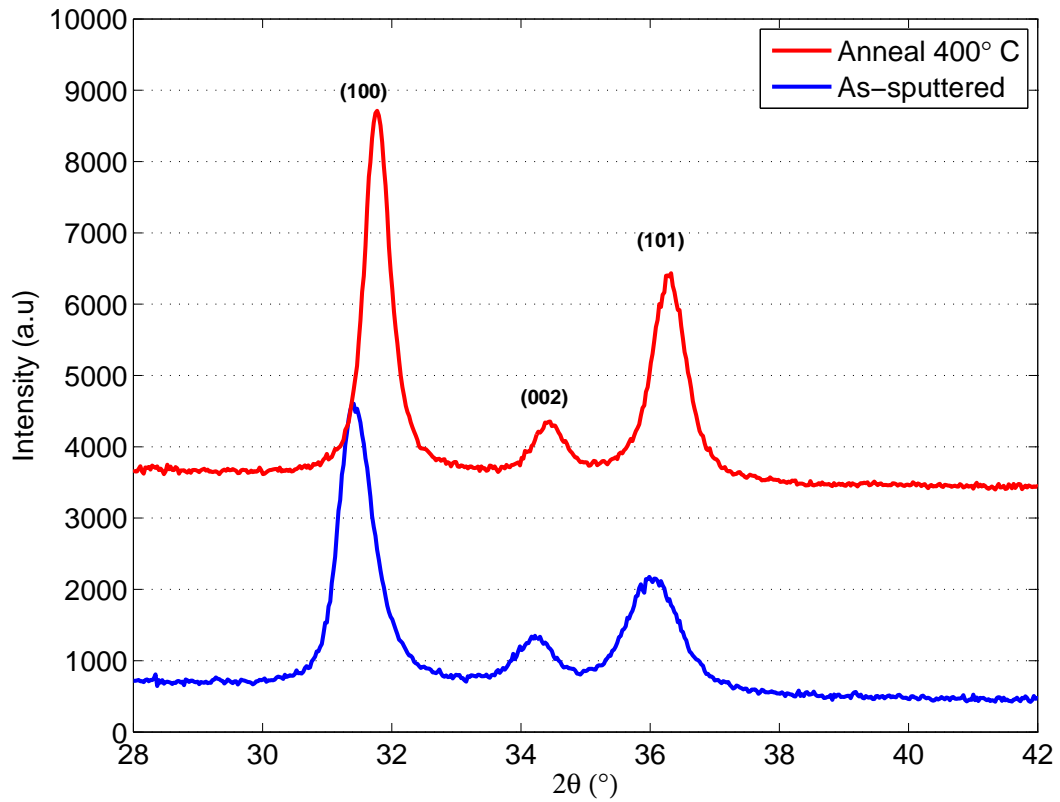

(b)

Figure 4.36: Initial annealing test performed on a hot plate at $400^{\circ} \mathrm{C}$ for 1 hour in air. (a) A film with (001) orientation prior to annealing, and (b) A polycrystalline film prior to annealing. 
A more sophisticated annealing technique involving an annealing furnace was then used. Samples to be annealed were placed inside a quartz tube and the tube placed inside an annealing furnace, under a flow of Ar: $\mathrm{O}_{2}$ of $95: 5 \%$ (described in Section 3.3).

A series of annealing experiments were performed at $250^{\circ} \mathrm{C}, 400^{\circ} \mathrm{C}$, $600^{\circ} \mathrm{C}, 750^{\circ} \mathrm{C}$ and $900^{\circ} \mathrm{C}$ using this annealing furnace. A film was cut into 5 smaller samples and XRD spectra for each sample was measured before and after annealing so that a comparison could be made. Figure 4.37 compares the XRD spectra for the as-sputtered and annealed films at the annealing temperatures of $250^{\circ} \mathrm{C}, 400^{\circ} \mathrm{C}$ and $600^{\circ} \mathrm{C}$. The Figure also shows the surface morphology images at the associated annealing temperature, where Figure 4.37a shows the surface morphology of an as-sputtered film. The main XRD results are also presented in Table 4.3.

It is clear from the XRD spectra's that all films undergo some stress relaxation, with films becoming less stressed with increasing annealing temperature. Although the films annealed at $250^{\circ} \mathrm{C}$ and $400^{\circ} \mathrm{C}$ show a slight increase in (002) peak intensity, there is also a small increase in the FWHM values. When annealing at $600^{\circ} \mathrm{C}$ the peak intensity has greatly increased and the FWHM is significantly lower than the as-sputtered film, suggesting an improvement in crystallinity. With increasing annealing temperature there is also some change in surface morphology. The surface morphology of the as-sputtered film (Figure 4.37a) shows well defined grain boundaries. However, the film annealed at $600^{\circ} \mathrm{C}$ shows less defined grain boundaries as the grains are beginning to merge. 


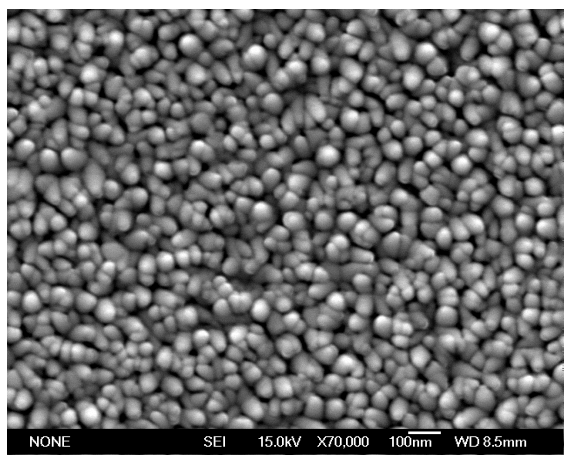

(a)

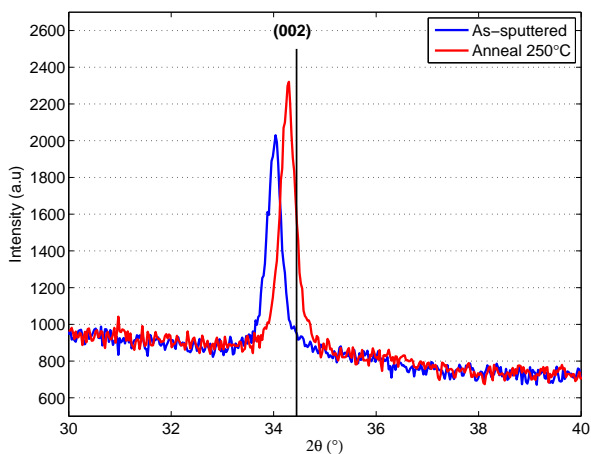

(b)

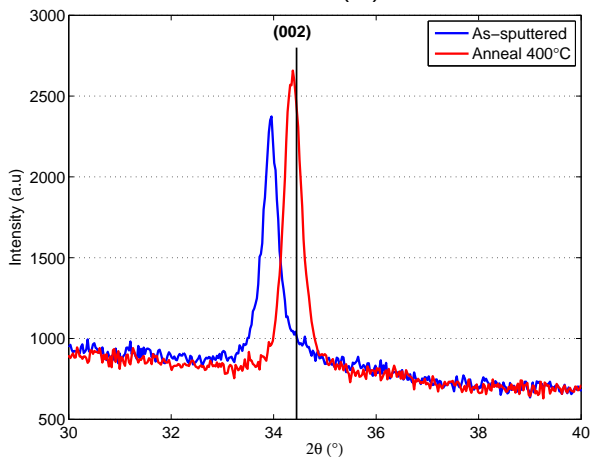

(d)

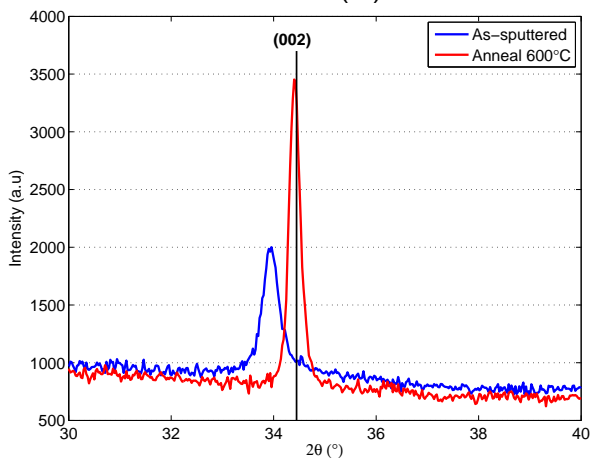

(f)

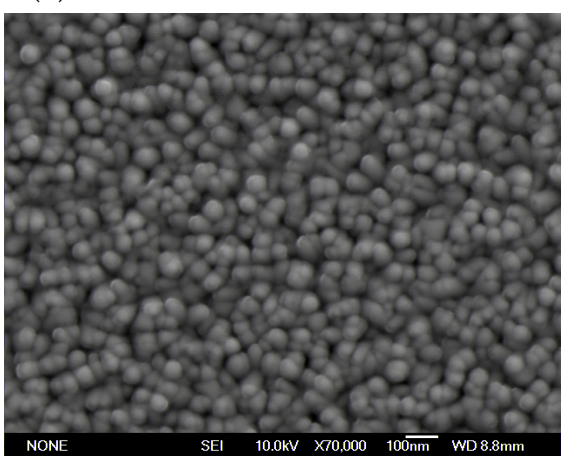

(c)

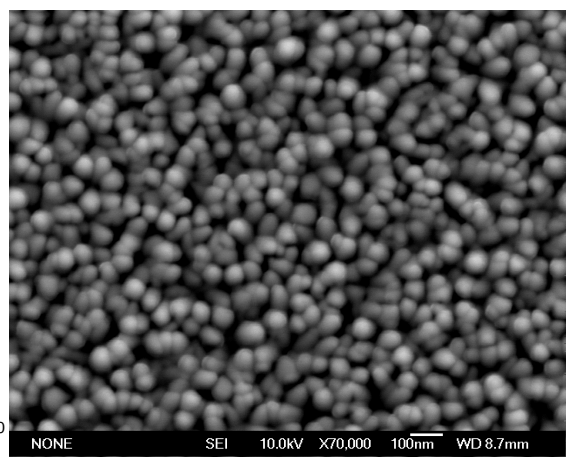

(e)

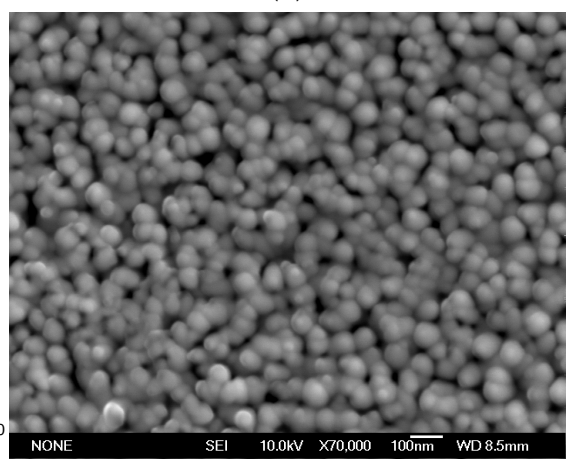

(g)

Figure 4.37: Crystal quality and surface morphology for annealed $\mathrm{ZnO}$ films: (a) Surface morphology of an as-sputtered film, (b) XRD spectra of the film as-sputtered and annealed at $250^{\circ} \mathrm{C}$, (c) Surface morphology after annealing at $250^{\circ} \mathrm{C}$, (d) XRD spectra of the film as-sputtered and annealed at $400^{\circ} \mathrm{C}$, (e) Surface morphology after annealing at $400^{\circ} \mathrm{C}$, (f) XRD spectra of the film as-sputtered and annealed at $600^{\circ} \mathrm{C}$, and (g) Surface morphology after annealing at $600^{\circ} \mathrm{C}$. 


\begin{tabular}{|c|c|c|c|c|}
\hline & $(002) 2 \theta\left(^{\circ}\right)$ & FWHM $\left.^{\circ}\right)$ & Peak intensity (a.u.) & Stress (GPa) \\
\hline As-sputtered & 34.01 & 0.338 & 1063 & -2.73 \\
Annealed at $250^{\circ}$ & 34.27 & 0.412 & 1326 & -1.00 \\
\hline As-sputtered & 33.93 & 0.338 & 1334 & -3.25 \\
Annealed at $400^{\circ}$ & 34.38 & 0.416 & 1717 & -0.31 \\
\hline As-sputtered & 33.93 & 0.364 & 1006 & -3.25 \\
Annealed at $600^{\circ}$ & 34.40 & 0.260 & 2421 & -0.14 \\
\hline As-sputtered & 34.06 & 0.312 & 1230 & -2.38 \\
Annealed at $750^{\circ}$ & 34.40 & 0.234 & 876 & -0.14 \\
\hline As-sputtered & 34.01 & 0.312 & 1411 & -2.73 \\
Annealed at $900^{\circ}$ & N.A. & N.A. & N.A. & N.A. \\
\hline
\end{tabular}

Table 4.3: Summarising the main XRD results from the as-sputtered films and after annealing.

It is interesting to notice that annealing at temperatures $>600^{\circ} \mathrm{C}$ does not further improve the film structure, but instead leads to a degradation in film quality. Figure 4.38 and 4.39 shows the annealing results at $750^{\circ} \mathrm{C}$ and $900^{\circ} \mathrm{C}$. In Figure 4.38a the XRD spectra indicates some stress relation and a reduction in the FWHM value compared to the as-sputtered; however there is degradation of the (002) peak and the introduction of some new undetermined peaks. The surface morphology images show a discontinuous structure with the film being pulled apart leaving large voids. This effect becomes more extreme when annealing at $900^{\circ} \mathrm{C}$.

As can be seen from the SEM images in Figure 4.39, the surface of the film is no longer a continuous structure after annealing at $900^{\circ} \mathrm{C}$. Some remnant of the $\mathrm{ZnO}$ film can be seen in Figure $4.39 \mathrm{c}$, however the majority of the surface consists of the structure shown in Figure $4.39 \mathrm{~d}$. These results are also indicated by the XRD spectrum shown in Figure 4.39e. The (002) peak has completely deteriorated.

This effect of annealing at $750^{\circ} \mathrm{C}$ and $900^{\circ} \mathrm{C}$ was suspected to be either the result of $\mathrm{ZnO}$ film being reduced to $\mathrm{Zn}$, or a solid state reaction between the $\mathrm{ZnO}$ film and glass substrate. Because the $\mathrm{ZnO}$ film becomes 
compromised, annealing at these higher temperatures is impractical.

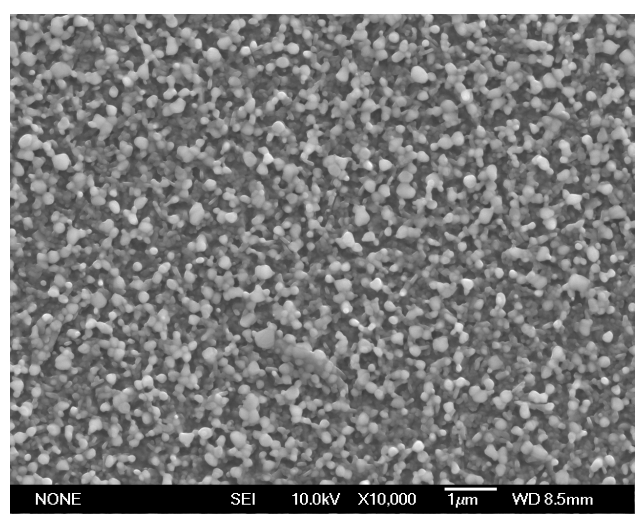

(a)

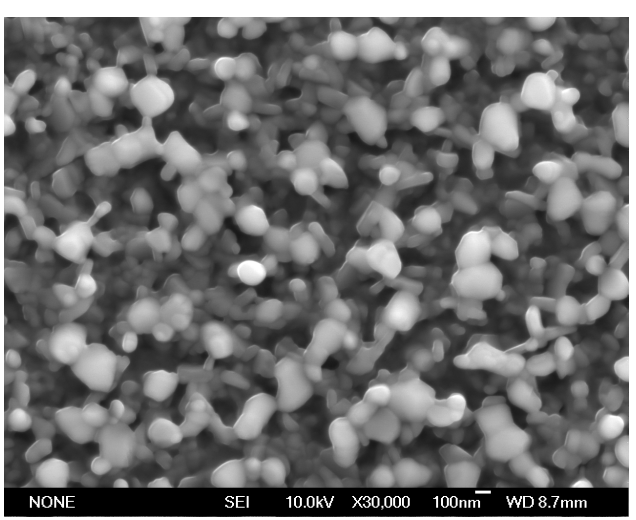

(b)

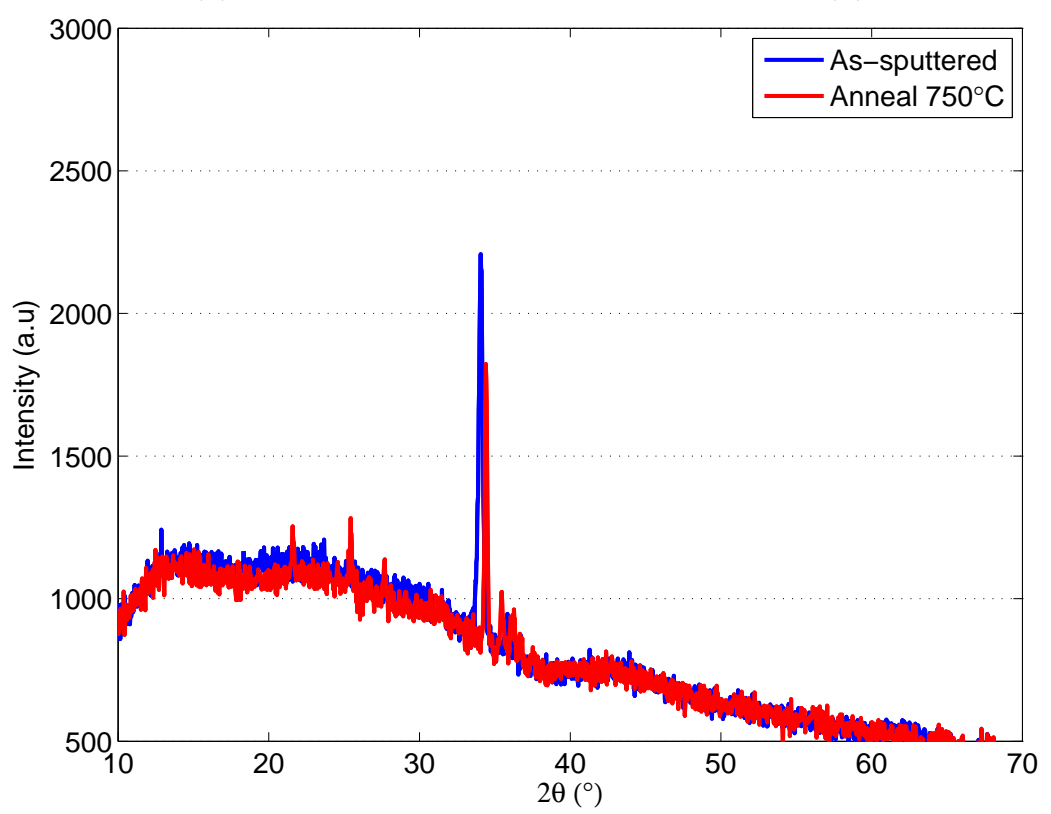

(c)

Figure 4.38: (a) and (b) Show surface morphology images of the annealed film at $750^{\circ} \mathrm{C}$, and (c) Compares the XRD spectra of the as-sputtered film and after annealing at $750^{\circ} \mathrm{C}$. 


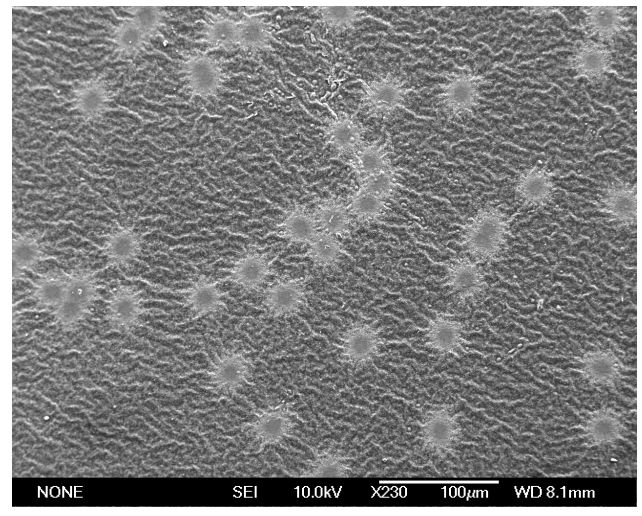

(a)

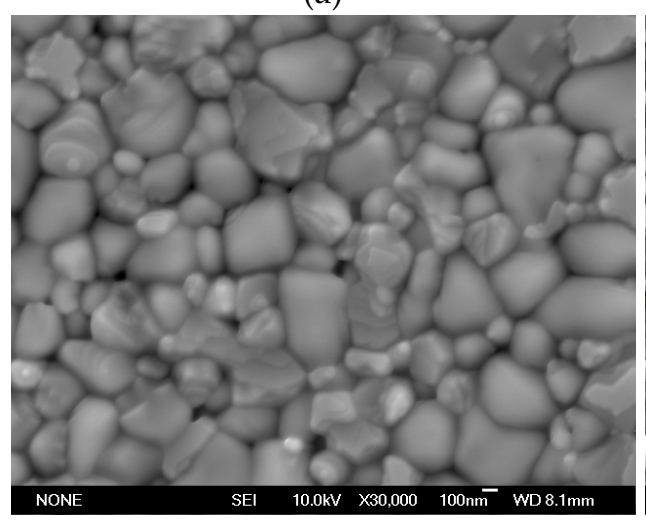

(c)

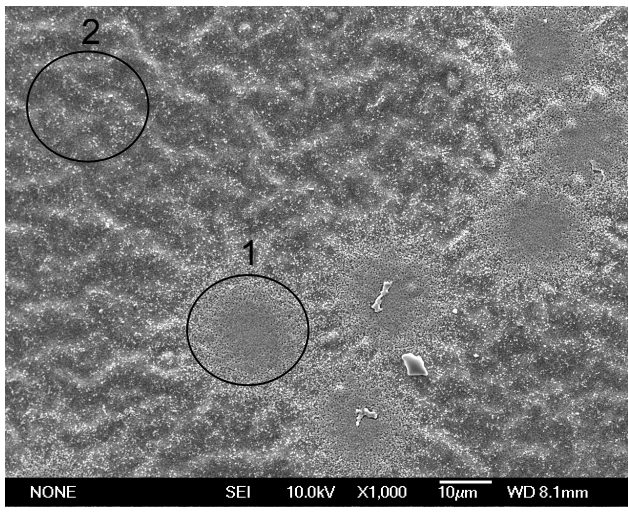

(b)

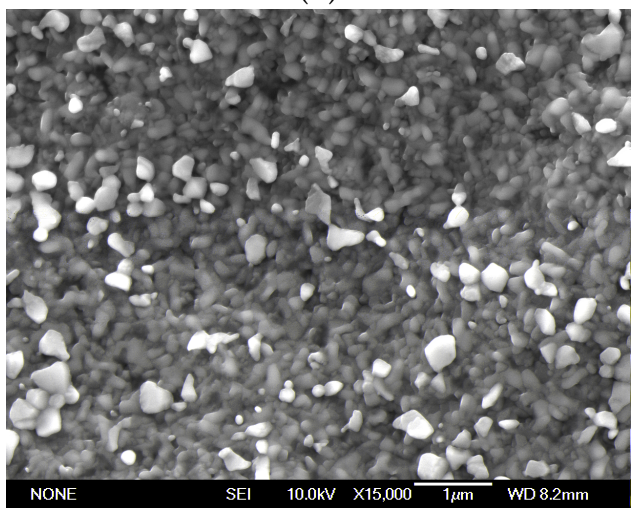

(d)

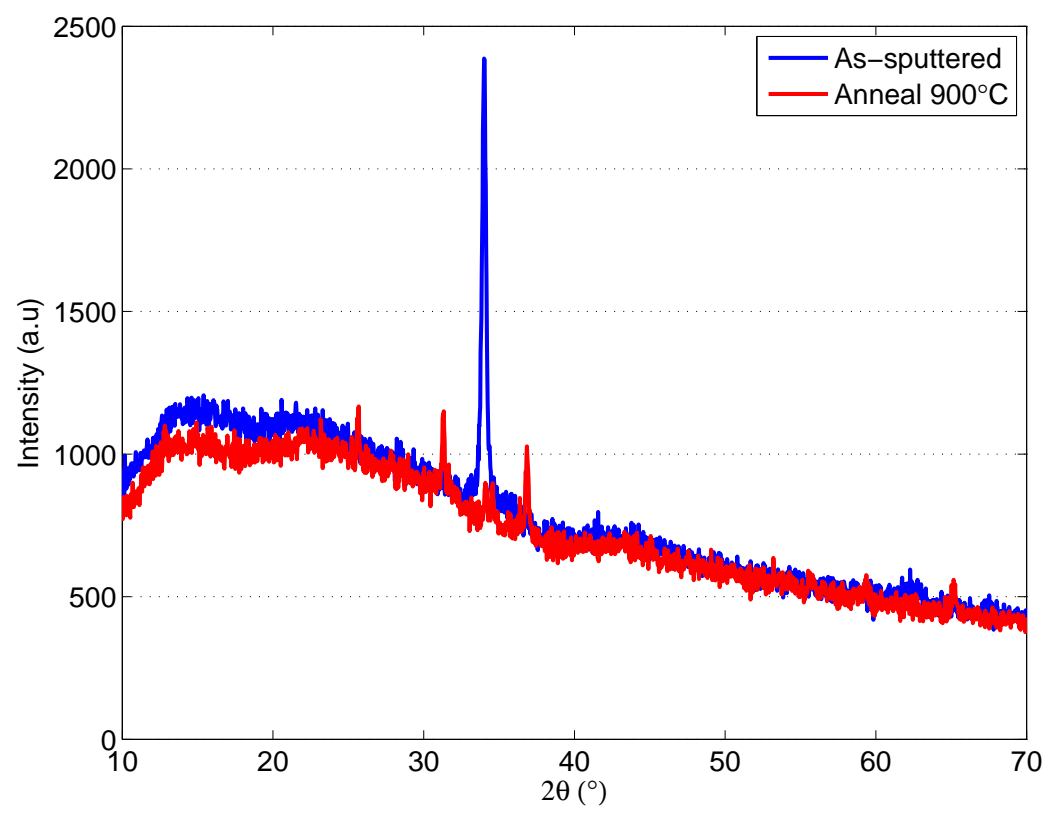

(e)

Figure 4.39: Surface morphology and $\mathrm{XRD}$ spectra of $\mathrm{ZnO}$ film annealed at $900^{\circ} \mathrm{C}$ : (a) Low magnification image of the films surface, (b) A close up image of the 'rounded' patches, Figures (c) and (d) Show high magnification images of circled areas 1 and 2 from Figure $b$ respectively, (e) XRD spectra of the as-sputtered film and after annealing at $900^{\circ} \mathrm{C}$. 
Figure 4.40 shows the influence of annealing temperature on the film stress and FWHM values as determined form the (002) reflection for the as-sputtered and annealed films. Upon annealing, the stress within the films decreased. At lower annealing temperatures there was very little change in the FWHM values, shown in Figure 4.40b. However, lower FWHM values were observed at $600^{\circ} \mathrm{C}$ and $750^{\circ} \mathrm{C}$, although at $750^{\circ} \mathrm{C}$ the initiation of the film deteriorating was observed. 


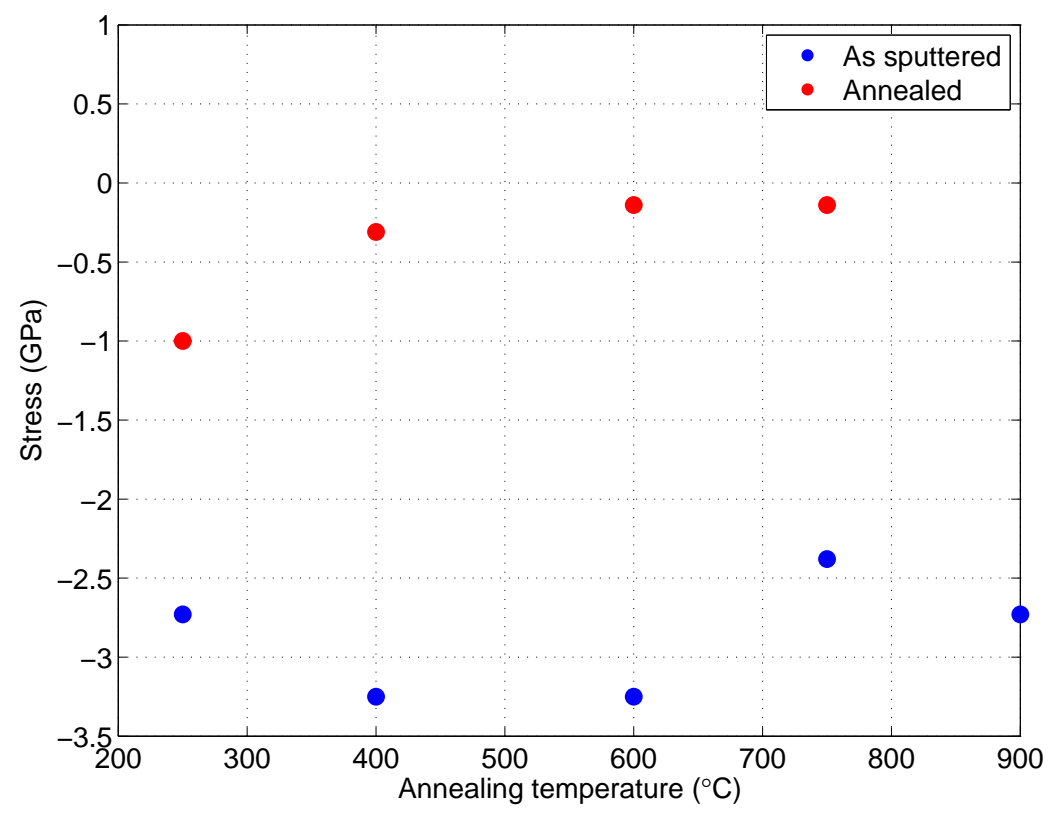

(a)

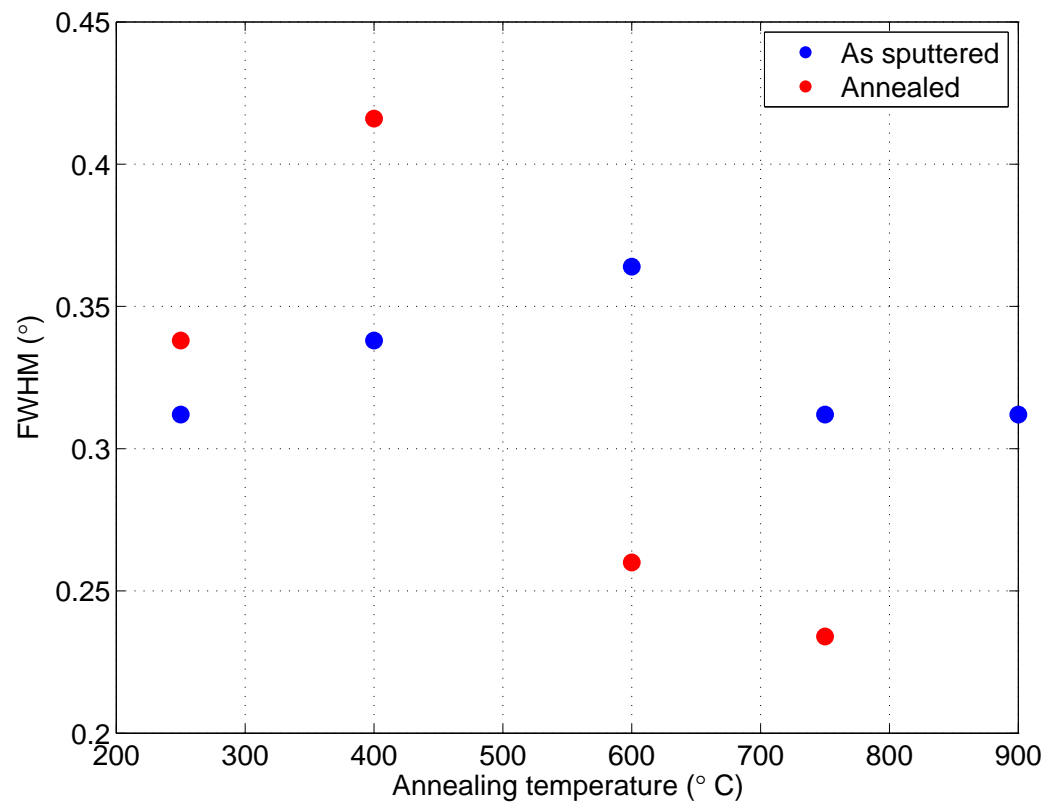

(b)

Figure 4.40: Comparing the effects of annealing temperatures on (a) The FWHM, and (b) The calculated stress. 


\subsubsection{Conclusions on stress within the $\mathrm{ZnO}$ films}

As the growth of thicker films for device applications began, it was observed that films began to crack and in extreme cases peel from the substrate surface. This effect was found to be the most severe at the central substrate position at the thickest region. A study of this phenomenon and possible solutions for it produced the following conclusions.

- Several mechanisms were considered as the cause of this phenomenon and inherent stress during the deposition process was determined to be the most likely cause.

- The influence of the deposition parameters on film stress was investigated. The deposition pressure was found to be the most influential, and at pressures below $1.2 \times 10^{-2}$ mbar peeling of the films was found. It has been observed for magnetron sputtering that there exists a dependence between working pressure and the intrinsic stress within a film [108]. As such, it was suggested that all subsequent films be deposited at pressures above $1.2 \times 10^{-2}$ mbar to reduce the intrinsic stress.

- Post growth annealing was found to be a successful technique in dramatically reducing the compressive stress within the films. An optimum annealing temperature of $600^{\circ} \mathrm{C}$ was found, as both the film stress and crystalline quality appears to be considerably improved at this temperature.

- Annealing at temperatures above $600^{\circ} \mathrm{C}$ did not improve the film structure, but rather led to deterioration of the film. This is potentially due to the reduction of $\mathrm{ZnO}$ to $\mathrm{Zn}$, or to a solid state reaction between the $\mathrm{ZnO}$ film and glass substrate. 


\subsection{Chamber modification \#2: New substrate holder}

The introduction of the substrate holder shaft extension enabled lower substrate-to-target distances. With a stationary substrate holder at a substrateto-target distance of $75 \mathrm{~mm}$ the deposition rate was dramatically increased. However, this was detrimental to the thickness uniformity of the resultant film. Films deposited on a substrate directly above the magnetron were the thickest, with a sharp decrease in thickness on other substrates. This was further complicated by the fact that the design of the original substrate holder made it very difficult to reliably position substrates symmetrically above the $\mathrm{ZnO}$ target. The potential design of a more effective substrate holder was investigated at this stage.

\subsubsection{Film uniformity}

In Figure 4.41 the position of 9 substrates of $25 \times 25 \mathrm{~mm}$ is shown mounted on the conventional substrate holder. Also projected onto this photograph is the approximate position of the $\mathrm{ZnO}$ target in relation to the substrate positions (dotted black line). The film thicknesses as measured by the Dektak are also indicated in Figure 4.41a, while this thickness data is turned into a surface plot in Figure 4.41a.

This information suggests that the region where the deposition rate is the fastest (shown in red) extends past where the substrates can be placed on the substrate holder. The thickness distribution appears to be non symmetric, this was the likely result of the mechanical design of the shutter, which always covered a small region on the target during a deposition. In an effort to improve uniformity and exploit the region where the deposition rate was the fastest these results led to the redesign of a second substrate holder which would place the substrates directly above the $\mathrm{ZnO}$ target. 


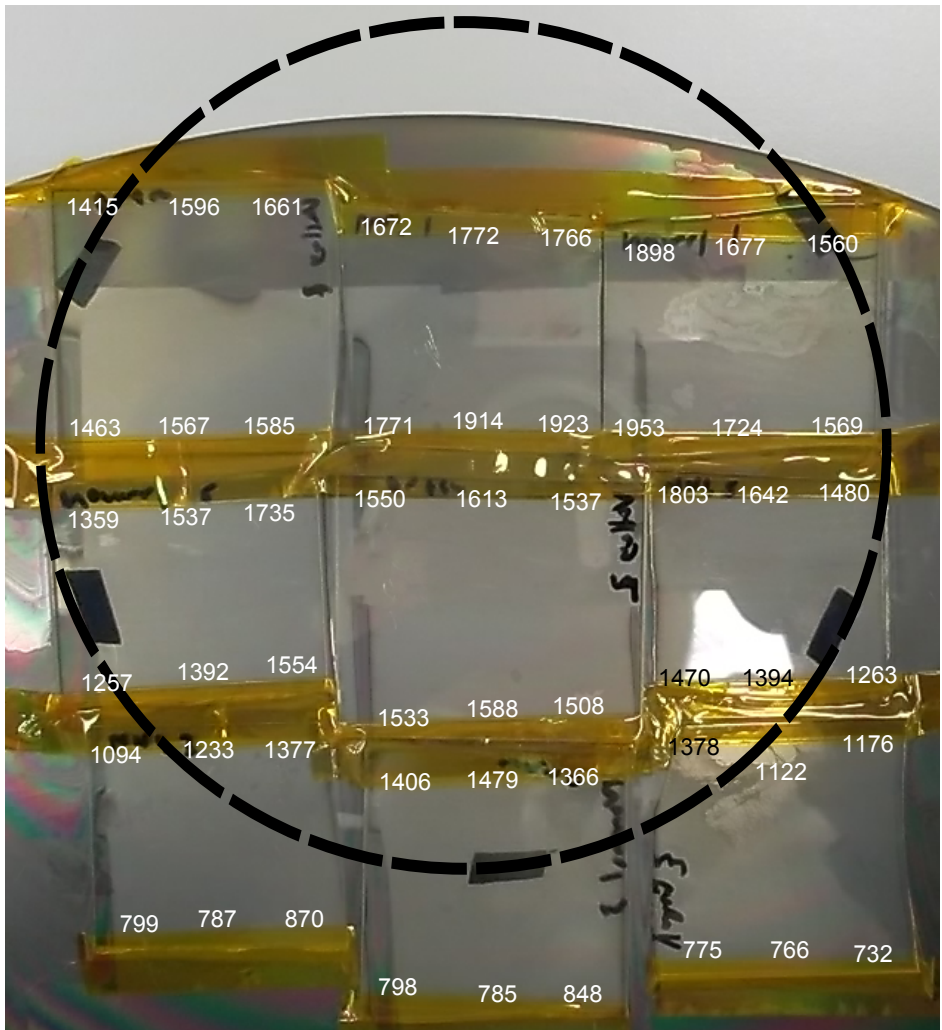

(a)

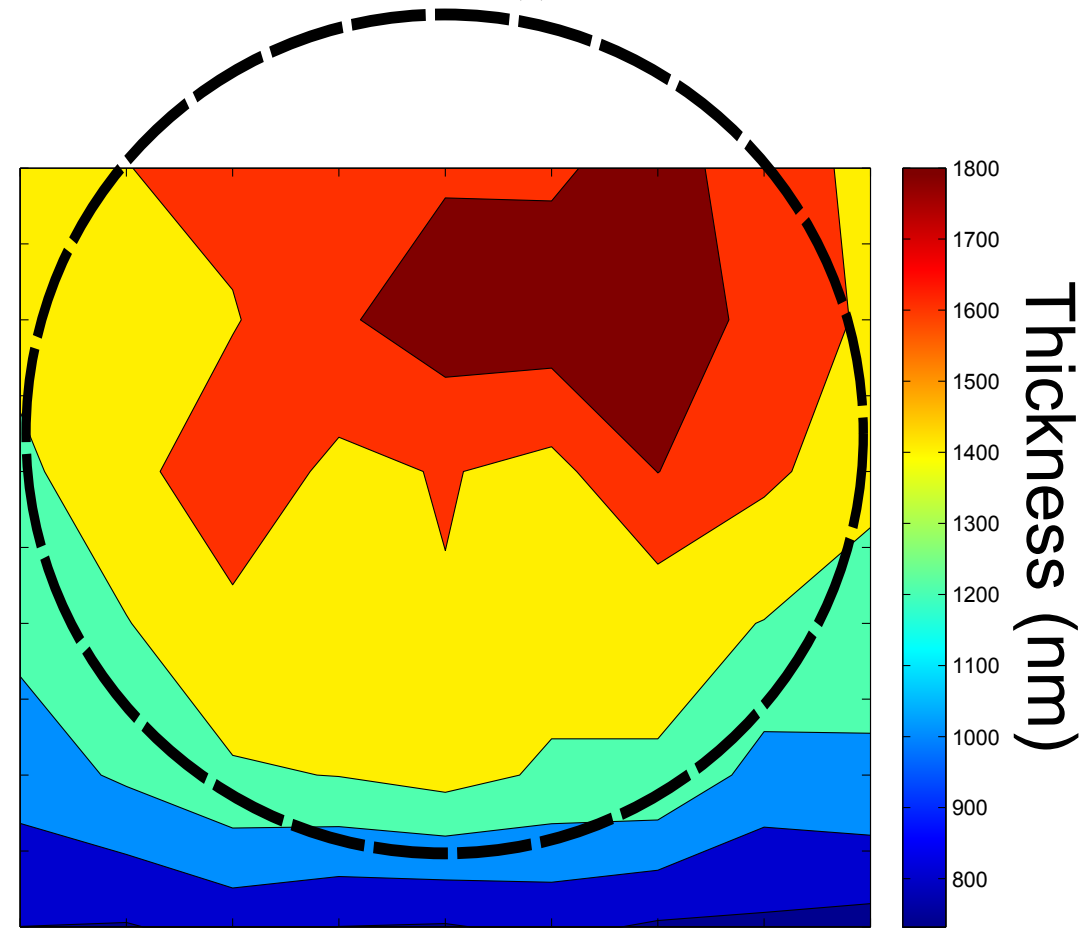

(b)

Figure 4.41: Demonstrating deposition rates and film uniformity across the substrates. (a) Shows the positions of the substrates with respect to the $\mathrm{ZnO}$ target and measured thicknesses, and (b) Expresses the thickness data in a surface plot. 
A second smaller substrate holder $(\approx 101 \mathrm{~mm}$ in diameter $)$ was designed to position the substrates directly above the $\mathrm{ZnO}$ target. This was attached via an arm and allowed the substrate holder to encompass the full area of the $\mathrm{ZnO}$ target. Figure 4.42 shows the new substrate holder inside the sputter chamber positioned $75 \mathrm{~mm}$ above the $\mathrm{ZnO}$ target. The new holder could take a single substrate $\leq 100 \mathrm{~mm}$ in diameter or up to nine smaller substrates of $25 \times 25 \mathrm{~mm}$ in size. The new chamber arrangement facilitated a repositioning of the quartz lamp to a position directly above the substrate holder. The thermocouple could be placed in a small groove inside the holder allowing for more accurate monitoring of the substrate temperature. Due to the design of this substrate holder it was not possible to rotate the substrate during depositions.

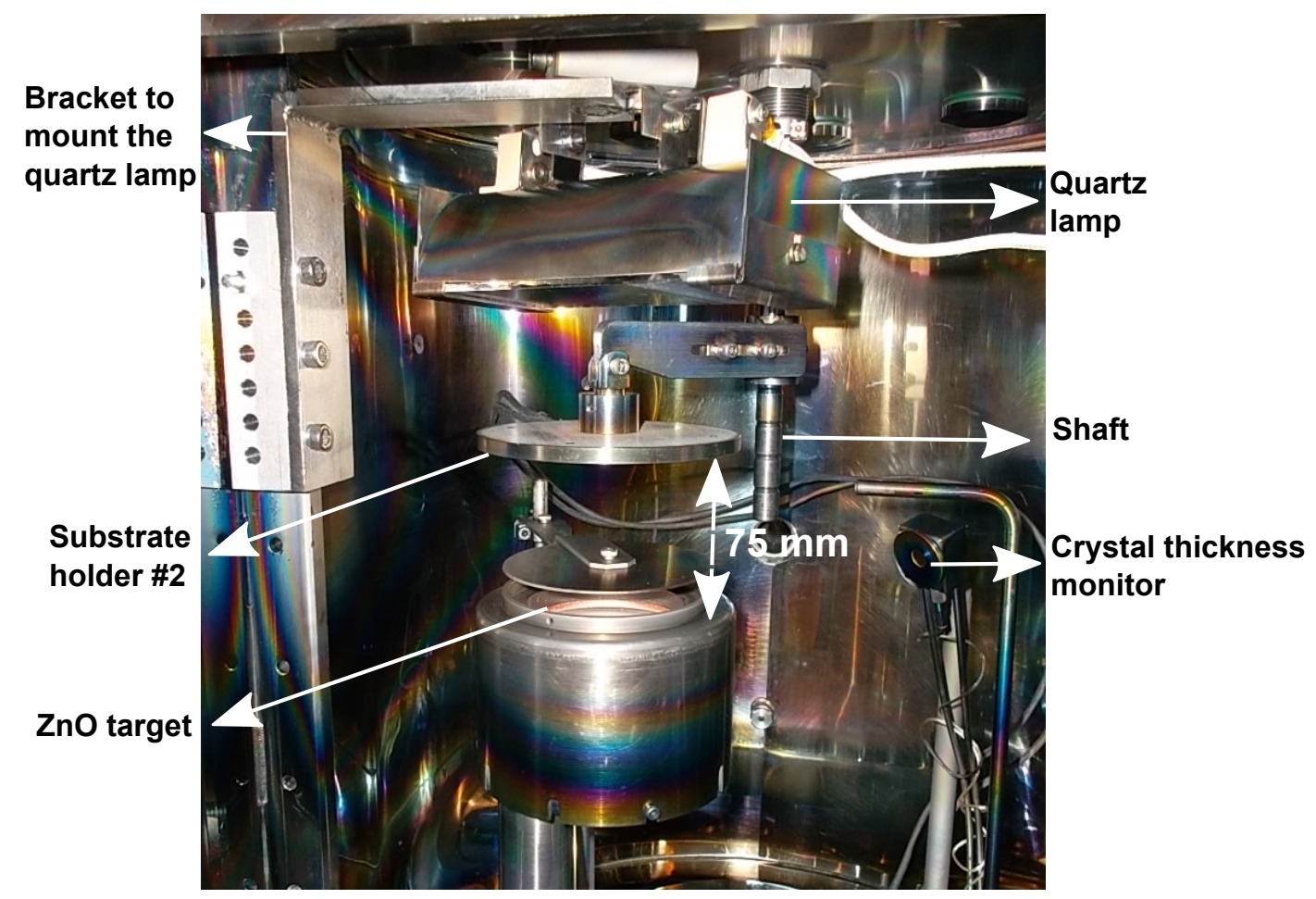

Figure 4.42: Inside the sputter coater: shows the new substrate holder attached to the shaft extension, substrate-to-target distance $75 \mathrm{~mm}$. The substrate heater is mounted to a bracket above the substrate holder. 
An initial deposition was performed to investigate the film uniformity using the new substrate holder. Nine, $25 \times 25 \mathrm{~mm}$ substrates were configured in a $3 \times 3$ matrix on the new substrate holder as shown in Figure 4.43 .

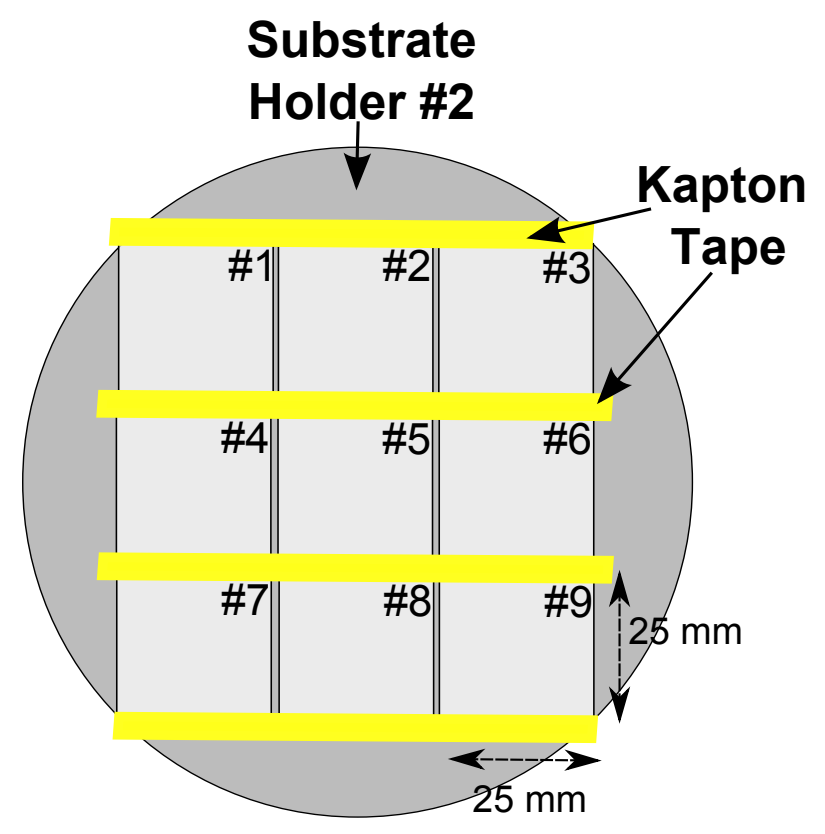

Figure 4.43: Substrate configuration using substrate holder \#2.

Figure 4.44a shows the thickness spread across the nine substrates using the new substrate holder, with the dotted line indicating the region of the target. As can be seen from Figure $4.44 \mathrm{~b}$ the film uniformity is greatly improved, with a larger central region of the substrate showing relatively uniform thickness. However, it is clearly noticeable that the thickness distribution is not symmetrical despite the fact that the substrate holder was placed symmetrically above the $\mathrm{ZnO}$ target. The reason for this was again suggested to be the effect of the shutter which covers part of the $\mathrm{ZnO}$ target during a deposition. The shutter opens to the bottom of the picture in Figure $4.44 \mathrm{~b}$, but it is suspected that it does not withdraw far enough. As a result it still influences the resultant shape of the plasma, causing a distortion of the deposition pattern. 


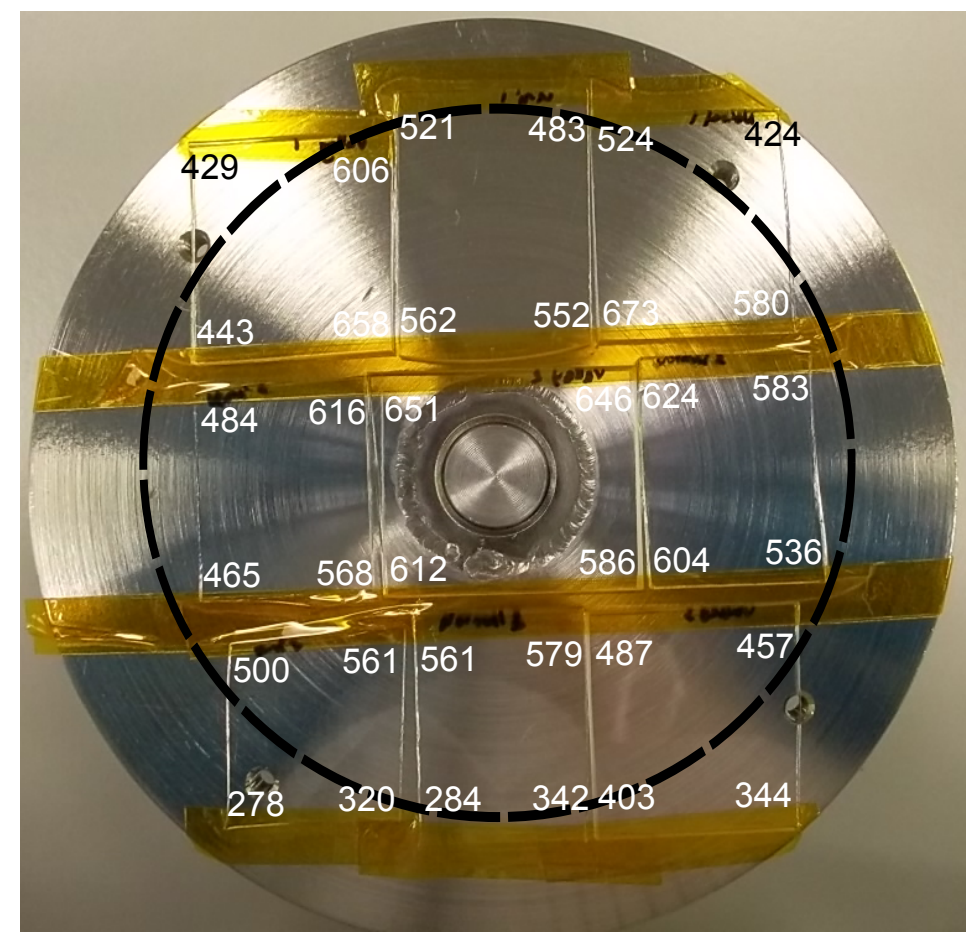

(a)

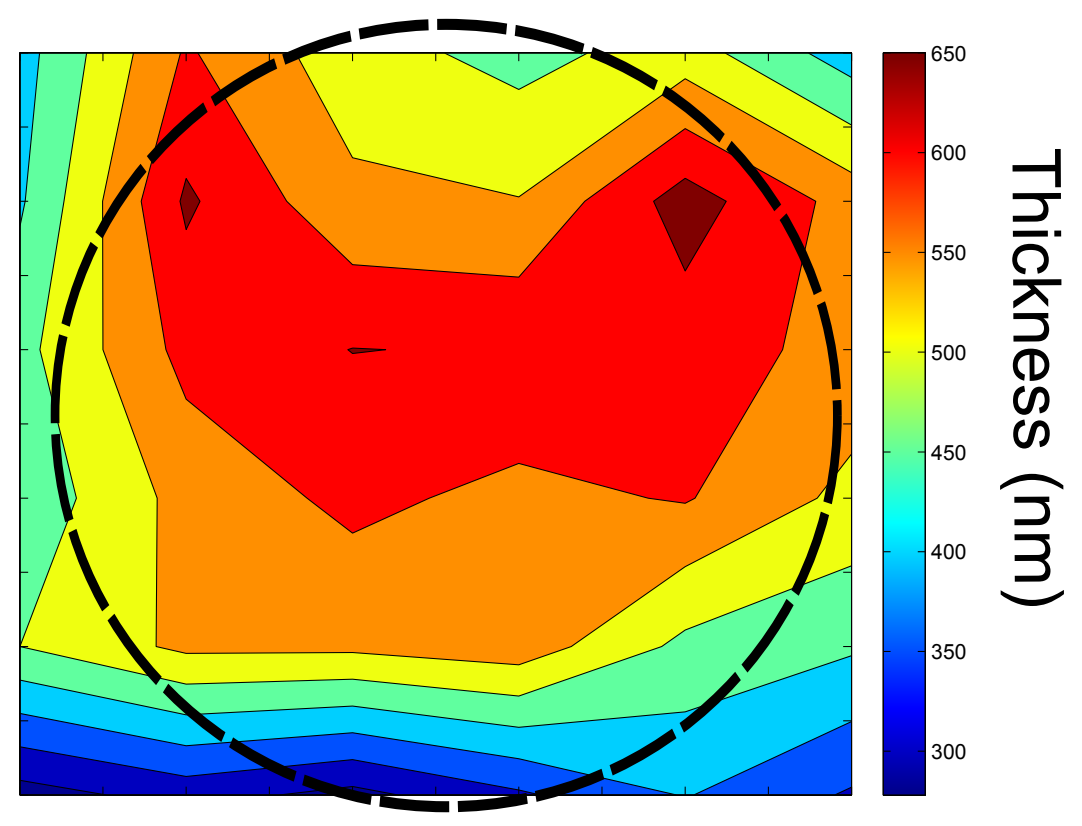

(b)

Figure 4.44: Demonstrating the film uniformity across the substrates using the second substrate holder. (a) Shows the positions of the substrates with respect to the $\mathrm{ZnO}$ target and measured thicknesses, and (b) Expresses the thickness data in a surface plot. 


\subsubsection{Conclusions from modification \#2}

This section evaluated the thickness uniformity of the conventional substrate holder which was found to be non uniform. A redesign of the substrate holder was investigated to improve thickness uniformity and the following conclusions drawn:

- With the introduction of the second substrate holder depositions are now performed at the fastest growth region in the plasma, and as such film uniformity has greatly improved.

- However, this thickness uniformity was found to be non symmetrical. It was suspected to be the result of the mechanical shutter, which when open, still covered a portion of the $\mathrm{ZnO}$ target during a deposition.

\subsection{Change in $\mathrm{ZnO}$ sputter target}

At this stage in the project, the $\mathrm{ZnO}$ target had become worn and was no longer suitable for depositions. A second $\mathrm{ZnO}$ target was purchased from the same supplier and used for all remaining depositions. It should also be noted that all depositions were performed above the critical pressure, $\approx 1.2 \times 10^{-2}$ mbar (described in Section 4.3.2).

\subsubsection{Change in optical appearance}

After the first deposition using the new $\mathrm{ZnO}$ target, the films appeared brownish in colour. This difference can be seen by conventional optical photographs shown in Figure 4.45 where a comparison is made between similar deposition performed using the old $\mathrm{ZnO}$ target (Figure 4.45a) and the new $\mathrm{ZnO}$ target (Figure 4.45b). 


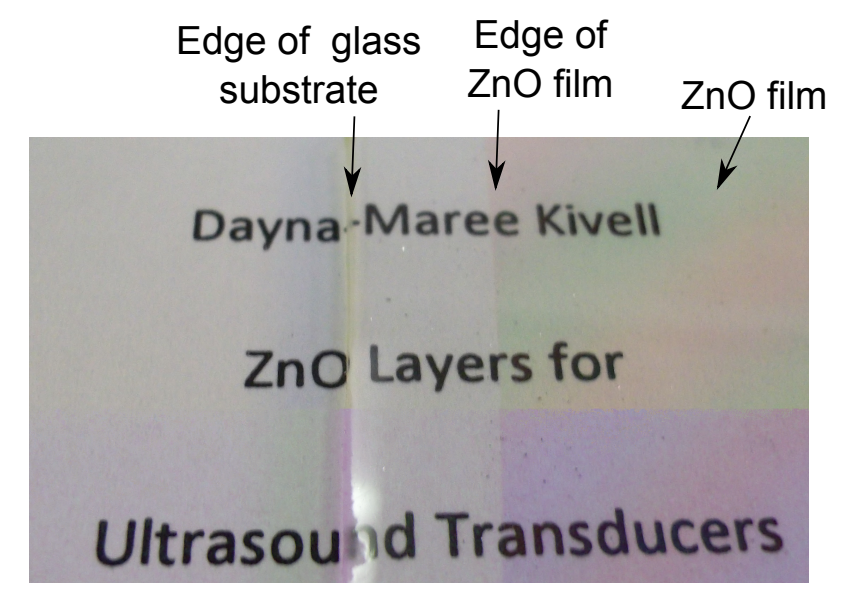

(a)

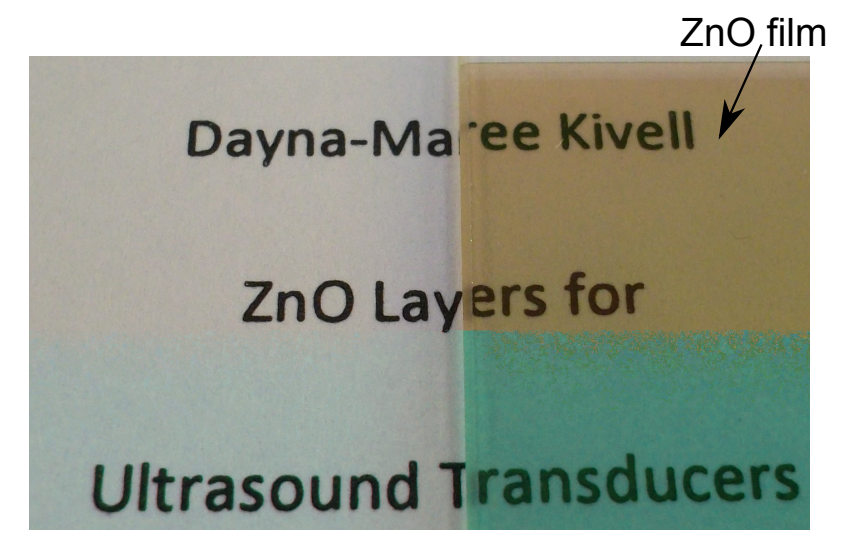

(b)

Figure 4.45: Comparing $\mathrm{ZnO}$ film colour between depositing with the (a) Old $\mathrm{ZnO}$ target appears clear in colour, and (b) New $\mathrm{ZnO}$ target appears brownish in colour. Shows the glass substrate with the deposited $\mathrm{ZnO}$ film to the right.

In Figure 4.46 a comparison is made between the crystal quality between two $\mathrm{ZnO}$ depositions, where one was performed using the old $\mathrm{ZnO}$ target and the other using the new $\mathrm{ZnO}$ target. The associated deposition rates for each spectrum was $\approx 15 \mathrm{~nm} / \mathrm{min}$ and both films were deposited using the two-step deposition process. The film deposited using the old $\mathrm{ZnO}$ target has a much higher (002) peak intensity, but also has a higher FWHM value. The reduced peak intensity when depositing using the new 
$\mathrm{ZnO}$ was typically observed.

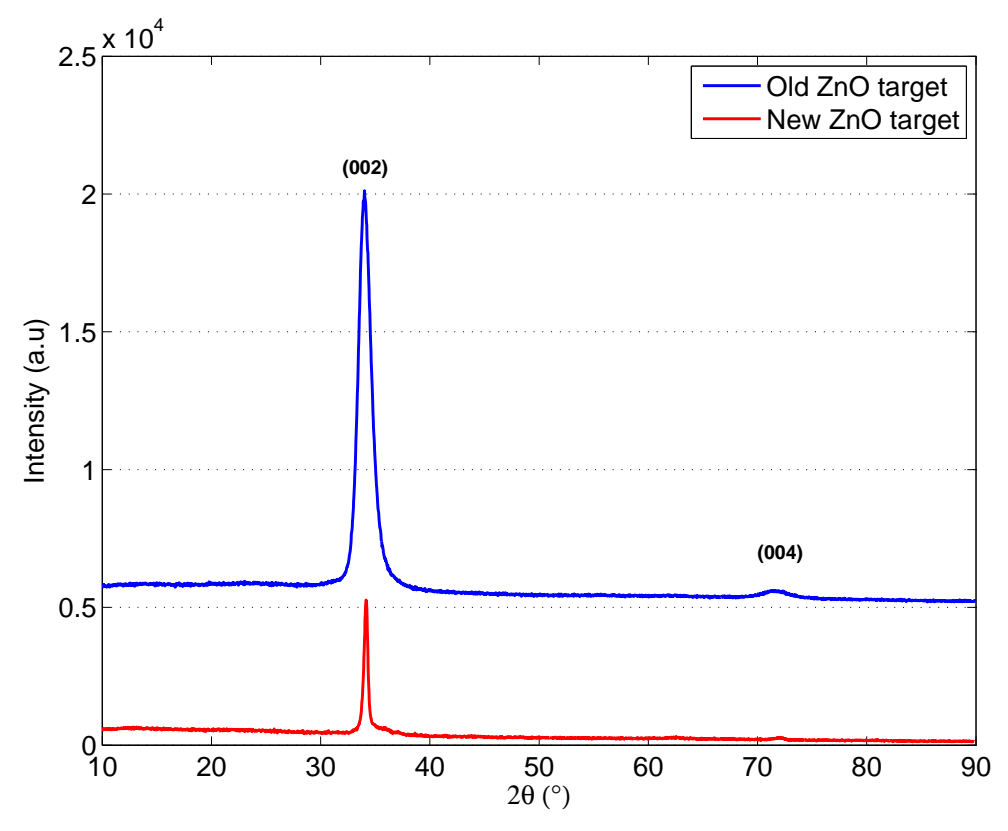

Figure 4.46: XRD comparison between the old $\mathrm{ZnO}$ target and the new $\mathrm{ZnO}$ target. The deposition rate for both films was $\approx 15 \mathrm{~nm} / \mathrm{min}$.

It was suspected that the film deposited using the new target had a depletion of oxygen from its lattice giving rise to the brownish colour. This potential lack of oxygen has been described by other workers due to the dissociation of the sputtered $\mathrm{ZnO}$ compound inside the plasma, creating zinc and oxygen atoms $[30,117]$. In principle these atoms will recombine at the surface of the substrate creating a $\mathrm{ZnO}$ layer. However, occasionally oxygen atoms will bond together forming $\mathrm{O}_{2}$ gas. As $\mathrm{O}_{2}$ gas does not have a unity sticking coefficient [118], it remains in this gaseous state and is removed from the chamber by pumping. As a result, there is depletion of oxygen and in turn an excess of zinc in the film. To compensate for this, oxygen can be flowed into the system along with the argon. The effect of an oxygen flow in addition to the Ar gas was then evaluated, for the new $\mathrm{ZnO}$ sputter target. 


\subsubsection{Effect of Ar: $\mathrm{O}_{2}$ ratio}

A series of depositions were performed at different Ar: $\mathrm{O}_{2}$ ratios of $100: 0$, $87.5: 12.5,75: 25$ and $62.5: 37.5$, where the ratios are expressed as the relative ratio of the gas flow rates with the total flow constant at $6 \mathrm{sccm}$. Figure 4.47 and Figure 4.48 show the UV-visible spectrophotometer transmission spectra and optical photographs of the films deposited at the various $\mathrm{Ar}: \mathrm{O}_{2}$ ratios, respectively. When depositing in pure Ar there is a drop in transmission, as shown in Figure 4.47a, which can be seen by the film colour shown in Figure 4.48a. With the presence of a small amount $\mathrm{O}_{2}$, as small as $12.5 \%$ very high transmission is observed, and subsequently for increasing $\mathrm{O}_{2}$. The films become clear in colour as shown by Figures $4.48 \mathrm{~b}$, $4.48 \mathrm{c}$ and $4.48 \mathrm{~d}$

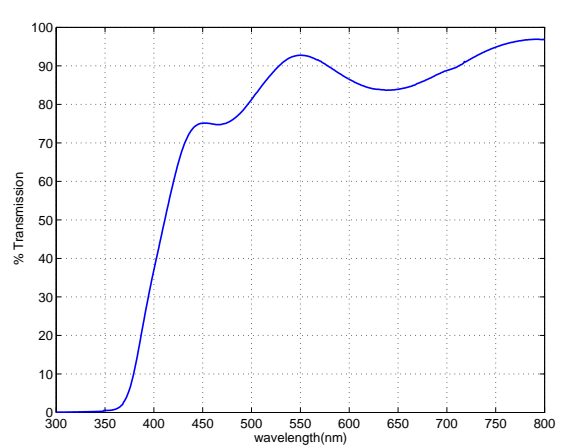

(a)

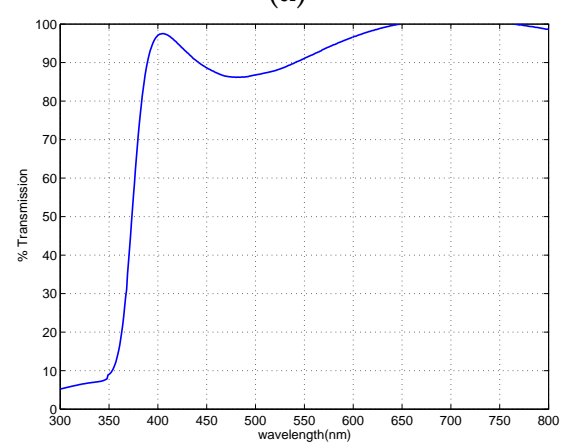

(c)

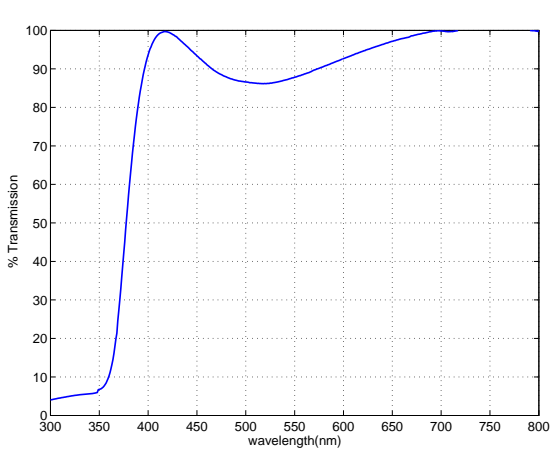

(b)

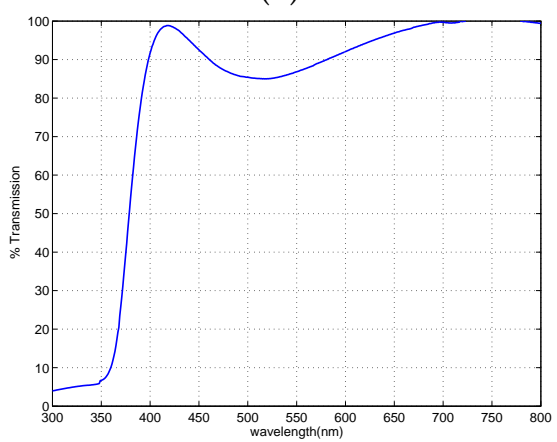

(d)

Figure 4.47: Comparing the UV-visible transmission for the various Ar: $\mathrm{O}_{2}$ ratios: (a) 100:0, (b) $87.5: 12.5$, (c) 75:25, and (d) 62.5:37.5. 


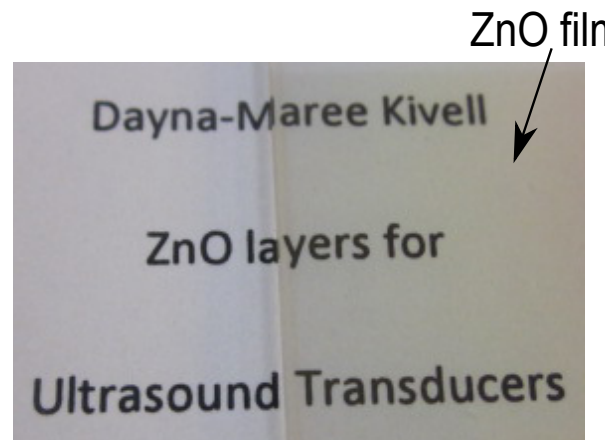

(a)

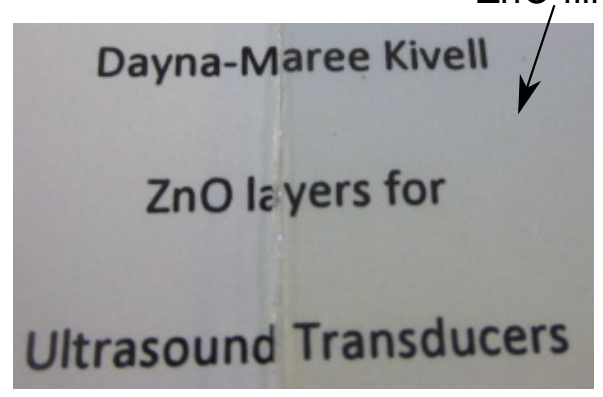

(c)

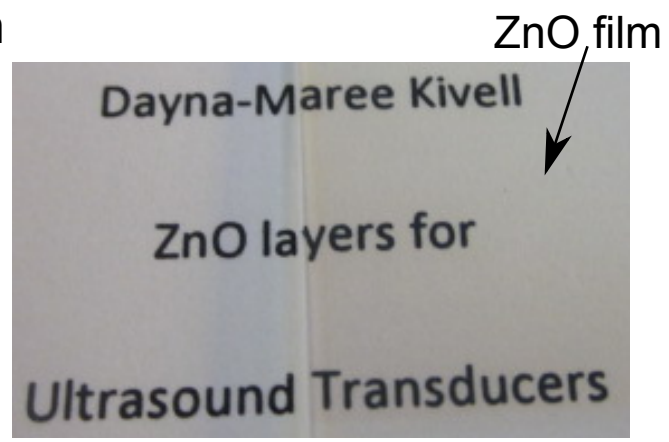

(b)

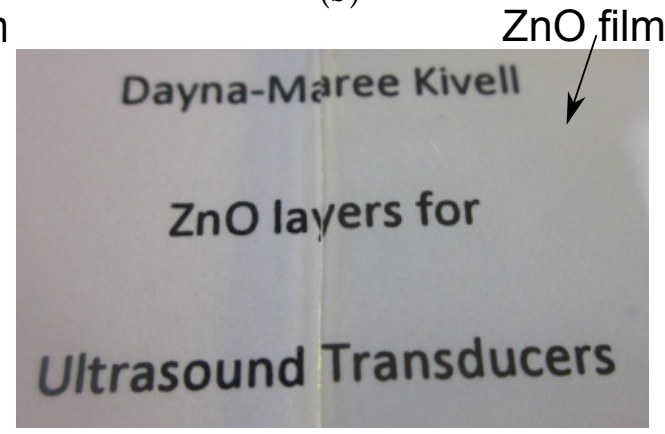

(d)

Figure 4.48: Comparing $\mathrm{ZnO}$ film colour when depositing at $\mathrm{Ar}: \mathrm{O}_{2}$ ratios (a) 100:0, (b) $87.5: 12.5$, (c) 75:25, and 62.5:37.5. Shows the glass substrate with the deposited $\mathrm{ZnO}$ film to the right.

The crystalline qualities of the films, based on the XRD measurements are shown in Figure 4.49. The result is surprising, as it appears that with the introduction of $\mathrm{O}_{2}$ there is a progressive deterioration in film quality. The (001) orientation is maintained, but the (002) peak shows a drop in intensity and a broadening in the FWHM value as the ratio of $\mathrm{O}_{2}$ is increased. However, the deposition rates were lower (and therefore the films thinner) when depositing in the presence of $\mathrm{O}_{2}$ which may be a contributing factor to the low (002) peak intensities. 


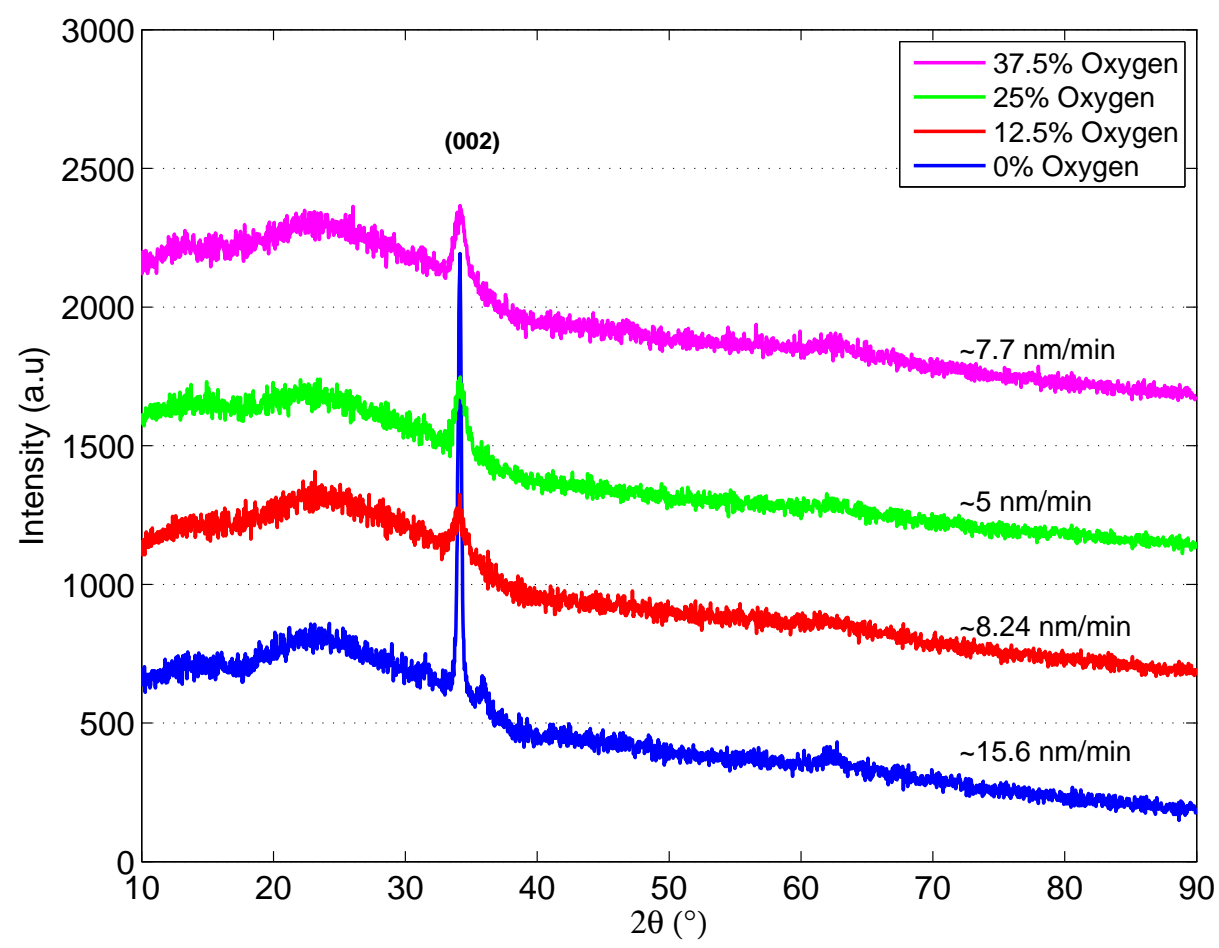

Figure 4.49: Influence of $\mathrm{Ar}: \mathrm{O}_{2}$ ratios on crystal quality. All other parameters held constant: RF power $=150 \mathrm{~W}$ and sputtering time of 20 mins.

\subsubsection{Other deposition parameters}

The influence of the RF power and substrate temperature was also investigated with the new $\mathrm{ZnO}$ target and very similar results found to those in Section 4.2. When depositing at higher deposition rates and at high substrate temperatures the quality of the films degraded, with degradation in the (002) peak intensity and the introduction of extra orientations.

\subsubsection{Conclusions from change in $\mathrm{ZnO}$ target}

During these experiments a second $99.99 \%$ purity $\mathrm{ZnO}$ target was used, however there appeared to be some discrepancies in results between the two $\mathrm{ZnO}$ targets. 
- Using the new $\mathrm{ZnO}$ target and depositing in a pure Ar chamber resulted in films being brown in colour. With the introduction of oxygen the films became clear again, suggesting that films deposited in pure Ar were oxygen deficient.

- When comparing the crystal quality between a deposition performed using the old $\mathrm{ZnO}$ target and the new $\mathrm{ZnO}$ target, films deposited with the new target showed lower (002) peak intensities, but smaller FWHM values.

- Although transparency was improved when depositing in an Ar to $\mathrm{O}_{2}$ mixed chamber, the quality of the films was compromised. Depositing in a pure Ar chamber showed the best quality $\mathrm{ZnO}$ films.

- The influence of RF power and substrate heating on film quality was found to be similar to that in Section 4.2, with films degrading at higher deposition rates and higher substrate temperatures.

- During the investigation of the sputtering parameters using the new $\mathrm{ZnO}$ target all depositions were performed above the critical pressure $\left(>1.2 \times 10^{-2}\right.$ mbar $)$. As a result, no cracking or delamination of the film from the substrate was observed. 


\section{Chapter 5}

\section{Conclusions and Suggestions for future work}

This chapter will summarise the main results of the thesis, as well as discuss some suggestions for future work that have been identified. It will begin by discussing the performance of the HHV Auto500 sputter coater system as well as the modifications which were made during the course of this research. Some further improvements to the sputter system will be suggested. The second part of this chapter discusses the results of our $\mathrm{ZnO}$ deposition on glass and attempts to suggest a simple model that explains the observed microstructure on deposition parameters, together with some suggestions for further characterisation of the films

\subsection{Performance and modification of the sputter system}

- The initial chamber geometry of the HHV Auto500 sputter coater system was unsuitable for our application due to the very low deposition rates. This was mainly due to the large target to substrate distance $(150 \mathrm{~mm})$ and was exacerbated when substrate rotation was 
used. Deposition rates were improved by extending the shaft of the substrate holder which reduced the distance between the target and substrate to $75 \mathrm{~mm}$, and by not using substrate rotation. However, at the lower target-to-substrate distance the film uniformity was very poor when not using substrate rotation.

- A redesign of the substrate holder placed substrates directly above the $\mathrm{ZnO}$ target which greatly improved uniformity. This also facilitated a repositioning of the quartz lamp which allowed a maximum substrate temperature of $250^{\circ} \mathrm{C}$ to be reached.

- Although deposition uniformity is improved by the new substrate holder, the deposition pattern is still non symmetrical. The most likely cause was the influence of the target shutter on the plasma distribution.

- Accurate and repeatable settings of the high vacuum valve position in order to set the chamber pressure during a deposition proved to be problematic. The importance of this setting was found to be critical for stress control in the $\mathrm{ZnO}$ films. Although no satisfactory alternative was introduced during this project, a more elegant solution is suggested below.

- Although not directly related to the sputter system, the variation in $\mathrm{ZnO}$ target material was found to be a potential problem. The second $\mathrm{ZnO}$ target appeared to produce $\mathrm{ZnO}$ films deficient in oxygen and with generally poorer characteristics. 


\subsection{Suggestions for further modifications to the sputter system}

- A more sophisticated design of the substrate holder which would allow the substrates to rotate in the region of the plasma should be done. Such a design would improve thickness uniformity whilst maintaining high deposition rates. An integrated substrate heater could also be incorporated, allowing for higher substrate temperatures to be reached. This may be valuable for performing annealing of the films without removing them from the sputter chamber.

- An electric control over the high vacuum valve position, with position feedback would allow for better control of the deposition pressure. This will eliminate the run-to-run variation which comes from the manual setting of the high vacuum valve and will improve stress control during the deposition process.

- A redesign of the mechanical shutter operation, which would allow it to completely move away from the $\mathrm{ZnO}$ target should be done. This will allow a more symmetrical distribution of sputtered material on the substrates.

- A quality control test should be performed when new $\mathrm{ZnO}$ targets are purchased. This may be as simple as a deposition performed under a set of standard conditions, followed by an analysis of film properties. It may be ideal to extend these measurements to a chemical analysis of the films in order to identify non stoichiometry or impurities. Such a process should be done in collaboration with the $\mathrm{ZnO}$ vendor. It is also suggested that higher purity targets (99.999\%) be purchased in the future. 


\subsection{Summary of results of $\mathrm{ZnO}$ deposition pro- cess}

- The aim of this project was to produce c-axis oriented $\mathrm{ZnO}$ films at relatively high deposition rates. However, there was a trade-off between these two requirements, as good textured c-axis oriented films were only produced at relatively low deposition rates $<15 \mathrm{~nm} / \mathrm{min}$. At higher deposition rates the films were polycrystalline with several orientations present.

- The deposition rate was primary controlled by the RF power. Films deposited at relatively low RF powers showed strong (001) orientation. Higher RF powers led to the degradation in crystalline quality

- Lower substrate temperatures appear to favour the desired (001) orientation. This effect seemed to be contrary to many published results $[84,94]$.

- A two-step deposition process, consisting of an initial low RF power was found to be viable for the production of c-axis oriented $\mathrm{ZnO}$ film at high deposition rates (up to $\approx 28 \mathrm{~nm} / \mathrm{min}$ )

- Films with thicknesses $>1.5 \mu m$ were often found to crack and peel from the substrate surface. It was determined that the most likely cause was inherent stress from the deposition process.

- The pressure during the deposition was found to have a critical influence on film stress. It was observed that films deposited at pressures below $1.1 \times 10^{-2}$ mbar had a tendency to crack and peel, while films deposited above this critical pressure were intact. Similar results had been found in the literature which suggested that stress within films was reduced when depositing at higher deposition pressures $[110,112,113]$. As a result it was proposed that films be deposited above $1.2 \times 10^{-2}$ mbar to reduce stress. 
- Through post growth annealing the stress within the films was almost completely relaxed. An optimum annealing temperature of $600^{\circ} \mathrm{C}$ for 1 hour in an $\mathrm{Ar}: \mathrm{O}_{2}$ atmosphere was found.

\subsection{A simple model for $\mathrm{ZnO}$ on glass deposition}

The results of this work determined that, although c-axis oriented films (of 001 orientation) can be grown using the RF sputtering process, under some deposition conditions this is not the only orientation present. The desired (001) orientation is only preferentially formed at low substrate temperatures and at low deposition rates. The ability to obtain c-axis oriented growth at moderately high deposition rates thus proved to be difficult, as an increase in the deposition rate resulted in the degradation of the c-axis orientation, leading to polycrystalline films. A simple model for the observed trend in the $\mathrm{ZnO}$ microstructure with deposition rate is explained below.

In the case of an amorphous substrate (such as glass), there is little influence from the substrate on the orientation of the initial film. The crystallographic orientation of the initial nuclei should primarily be determined by the surface (faces) that are the most stable and are formed by the lowest surface free energy planes. In contrast, a crystalline substrate will influence the lattice of the film as it will try to conform to the lattice of the substrate.

In the case of thin film formation on glass substrates it can then be expected that the (001) nuclei will be preferentially formed, as this is the most stable surface for $\mathrm{ZnO}$ as can be seen in Figure 5.1a [119, 120]. However, it is expected that during the sputter deposition process, with the bombardment of high energy particles on the (001) nuclei, they may be knocked over, producing the (001) surface at an angle to the substrate surface. This knock over effect will become more important as the RF power is increased. Depending on the angles formed, a new surface plane will 
be exposed, with the resultant film surface forming the orientation of the crystallite, shown in Figure 5.1b. If the (001) nuclei falls parallel to the substrate surface, the (100) plane becomes exposed. This would explain the prominent contribution of this orientation to the $\mathrm{ZnO}$ films when depositing at the higher deposition rates. The effect of the growth and coalescence of nuclei forming different angles at the substrate surface will result in a film with a more uneven surface morphology than if all the nuclei had the (001) surface parallel to the substrate.
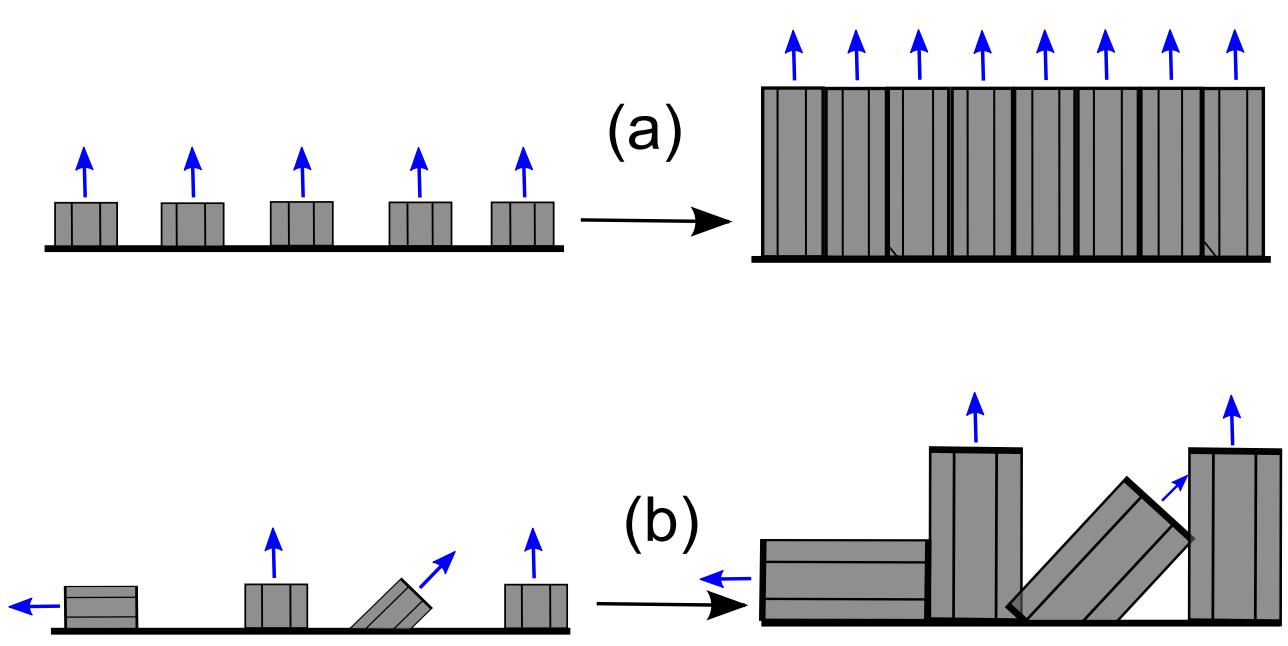

Figure 5.1: Illustrating the nucleation and growth stages (a) A deposition performed at a low deposition rate, films preferentially grow with the c-axis perpendicular to the substrate in the (001), and (b) A deposition performed at a higher deposition rate, resulting in random orientation. Where the blue arrow indicates the c-axis.

The surface morphology predicated by this model has been supported by SEM images of films. Depositing at low deposition rates allows (001) nucleation and growth process to occur with relatively smooth surfaces, while higher deposition rates produce very uneven surfaces with sharp edges. In Figures 4.28 and 4.29 it can be seen how these two types of surfaces are associated with two types of microstructure. 
The effect of the initial slow growth buffer layer consisting of a good quality (001) oriented film grown at low RF powers can now be appreciated. Once such a layer has formed, it will serve as a 'substrate' for further $\mathrm{ZnO}$ deposition. The interaction and bonding between this $\mathrm{ZnO}$ buffer and any subsequent $\mathrm{ZnO}$ nuclei will be much stronger than the interaction between an amorphous glass substrate and $\mathrm{ZnO}$ nuclei. This will prevent initial nuclei from being knocked over during higher deposition rates. The result of such a process is that the (001) orientation is maintained even at high deposition rates as was found during our two-step depositions.

\subsection{An optimum deposition process}

The above results provided an insight into the various deposition parameters which influenced the $\mathrm{ZnO}$ growth process. An optimised deposition process should then include the following steps:

Step 1: An initial buffer layer deposited at a low rate to facilitate a seed layer for good c-axis orientation.

Step 2: An in situ anneal step inside the sputter coater chamber to relieve stress within the buffer layer.

Step 3: Decrease the temperature to ambient growth temperature and deposit at a higher RF power to enhance the deposition rate.

Step 4: Further anneal the deposited film in order to remove stress from film before device processing.

To further improve crystallinity and reduce film stress, the in situ annealing processes could be performed in periodic cycles. However, such a process will slow down the device fabrication time. 


\subsection{Suggestions for future work}

Although the initial requirements of c-axis orientation at high deposition rates have been achieved by this project, these are not the only requirements for piezoelectric activity in $\mathrm{ZnO}$. The following work is then suggested for further characterisation of the films and refinement of the deposition process.

- Test the proposed four-step deposition procedure. Due to the erratic results from the second $\mathrm{ZnO}$ target, as well as the time limitations of the project this process had not yet been performed.

- The electric and piezoelectric properties of the films should be measured. Particularly measurements of the piezoelectric coefficient will provide valuable feedback for optimisation of the deposition process. A mask has been designed which allows for a simple device structure to be fabricated. Such structures would facilitate the measurement of the $\mathrm{ZnO}$ films dielectric constant, resistivity and frequency response.

- As well as c-axis orientation, the $\mathrm{ZnO}$ film surface should be of the same polarity. The (001) plane is a polar surface (with either a positive $\mathrm{Zn}$-polar face or a negative O-polar face), and the $\mathrm{ZnO}$ film surface should be of the same polarity for maximum piezoelectric response. An initial investigation into the polarity of the $\mathrm{ZnO}$ films surface has begun. Films were etched in hydrochloric acid (HCL), as each surface etches at a different rate, the etch rate can be observed through cross sectional SEM imaging. Initial results suggest that the film's surface was of the same polarity, however this technique should be further developed. 


\section{Bibliography}

[1] M.D.Ambersley and C.W.Pitt, "Piezoelectric $\mathrm{ZnO}$ transducers produced by RF magnetron sputtering," THIN SOLID FILMS, vol. 80, pp. 183-195, 1981.

[2] J.A.Galleo-Juárez, "Piezoelectric ceramic and ultrasound transducers," J PHYS E SCI INSTRUM, vol. 22, pp. 804-816, 1989.

[3] Y. Ito, K. Kushida, K. Sugawara, and H. Takeuchi, "A 100-MHz ultrasonic transducer array using ZnO thin films," IEEE T ULTRASON FERR, vol. 42, pp. 316-324, 1995.

[4] Y. Hsu and J. Lin, Tang, "RF sputtered piezoelectric zinc oxide thin film for transducer applications," J. MATER. SCI. MATER., vol. 19, pp. 653-661, 2007.

[5] G.-H. Feng, C. Sharp, Q. Zhou, W. Pang, E. Kim, and K. Shung, "Fabrication of MEMS $\mathrm{ZnO}$ dome-shaped-diaphragm transducers for high-frequency ultrasonic imaging,"

[6] Q. Su, P. Kirby, E. Komuro, M. Imura, Q. Zhang, and R. Whatmore, "Thin-film bulk acoustic resonators and filters using $\mathrm{ZnO}$ and leadzirconium-titanate thin films," IEEE T MICROW THEORY, vol. 49, pp. 769-778, 2001.

[7] K.M.Lakin, "Thin film resonator technology," IEEE T ULTRASON FERRl, vol. 52, pp. 707-716, 2005. 
[8] J. Tressler, S. Alkoy, and R. Newnham, "Piezoelectric sensors and sensor materials," J ELECTROCERAM, vol. 2, pp. 257-272, 1998.

[9] P. Muralt, R. Polcawich, and S. Trolier-McKinstry, "Piezoelectric thin films for sensors, actuators, and energy harvesting," MRS BULL, vol. 34 (9), pp. 658-664, 2009.

[10] W. Chang, Y. Chen, R. Lin, Cheng, K. Kao, B. Wu, and Y. Huang, "Design and fabrication of a piezoelectric transducer for windpower generator," THIN SOLID FILMS, vol. 519, pp. 4687-4693, 2011.

[11] G. Taylor, J. Gagnepain, T. RMeeker, T. Nakamura, and L. Shuvalov, Piezoelectricity - Ferroelectricity and Related Phenomena. Gordon and Breach Science Publishers, Vol 4, 1985.

[12] G.Gautschi, Piezoelectric Sensorics Force, Strain, Pressure, Acceleration and Acoustic Emission Sensors, Materials and Amplifiers. SpringerVerlag Berlin and Heidelberg Gmbh \& Co., 2002.

[13] I.J.Busch-Vishniac, Electromechanical Sensors and Actuators. SpringerVerlag New York Inc, 1999.

[14] A. M. Nicholson, “Piezophony,” U.S. Patent 1, 495, 429, May, 1924.

[15] G.Gerlach and W.Dötzel, Introduction to Microsystem Technology: A Guide for Students. John Wiley \& Sons Ltd., 2008.

[16] K. Shung, J. Cannata, and Q. Zhou, "Piezoelectric materials for high frequency medical imaging applications: A review," J ELECTROCERAM, vol. 19, pp. 139-145, 2007.

[17] K.K.Shung, Diagnostic ultrasound imaging and blood flow measurements. CRC Press Taylor and Francis group, 2005. 
[18] T. Shrout, "Innovations in piezoelectric materials for ultrasound transducers," in Medical Imaging 1998: Ultrasonic Transducer Engineering, 2008.

[19] A. Jakob, M. Bender, T. Knoll, R. Lemor, M. Bender, T. Lehnert, M. Koch, M. Veith, Q. Zhou, B. P. Zhu, J. X. Han, and K. K. Shung, "Comparison of different piezoelectric materials for $\mathrm{GHz}$ acoustic microscopy transducers," ULTRASON, pp. 1722-1725, 2009.

[20] P. Martin, M. Good, J. Johnston, G. Posakony, L. Bond, and S. Crawford, "Piezoelectric films for 100-MHz ultrasonic transducers," THIN SOLID FILMS, vol. 379, pp. 253-258, 2000.

[21] U. Özgür, Y. I. Alivov, C. Liu, A. Teke, M. A. Reshchikov, S. Doğan, V. Avrutin, S. J. Cho, and H. Morko, "A comprehensive review of ZnO materials and devices," J APPL PHYS, vol. 98, pp. 041301 1103, 2005.

[22] D. G. Thomas, "The excition spectrum of zinc oxide," J APPL PHYS, vol. 15, p. 86, 1960.

[23] I.-S. Jeong, J.-H. Kim, and S. Im, "Ultraviolet-enhanced photodiode employing n-ZnO/p-Si structure," APPL PHYS LETT, vol. 83, pp. 2946-2948, 2003.

[24] Z.Wang, "Zinc oxide nanostructures: growth, properties and applications," J PHYS-CONDENS MAT, vol. 16, pp. 829-858, 2004.

[25] Z.Wang, "Novel nanostructures of $\mathrm{ZnO}$ for nanoscale photonics, optoelectronics, piezoelectricity, and sensing," APPL PHYS A-MATER, vol. 88, 2007.

[26] A. Cheng, One Dimensional Zinc Oxide Nanostructures for Optoelectronic Applications: Solar cells and photodiodes. Auburn University, 2011. 
[27] W. Hu, Z. Liu, J. Sun, S. Zhu, Q. Xu, D. Feng, and Z. Ji, “Optical properties of pulsed laser deposited $\mathrm{ZnO}$ thin films," J PHYS CHEM SOLIDS, vol. 58, pp. 853-857, 1997.

[28] R.Triboulet and J.Perriére, "Epitaxial growth of $\mathrm{ZnO}$ films," PROG CRYST GROWTH CH, vol. 47, pp. 65-138, 2003.

[29] B. Wacogne, M. P. Roe, T. J. Pattinson, and C. N. Pannell, “Effective piezoelectric activity of zinc oxide films grown by radio frequency planar magnetron sputtering," J APPL PHYS, vol. 67, 1995.

[30] K. Ellmer, A. Klein, and B. Rech, Transparent Conductive Zinc Oxide: Basics and Applications in Thin Film Solar Cells. Springer, 2008.

[31] "Zinc (Zn)."Encyclopedia Britannica online. Retrieved from http://www.britannica.com/EBchecked/topic/657264/zincZn/8119/History, 2012.

[32] M. Ashby, P. Ferreira, and D. Schodek, Nanomaterials, Nanotechnologies and design: An introduction for Engineers and Architects. Elsevier Ltd., 2009.

[33] J.L.Garvin, A building History of Northern New England. University Press of New England, 2001.

[34] C.Jagadish and S.J.Pearton, eds., Zinc Oxide Bulk, Thin Films and Nanostructures: Processing, Properties and Applications. Elsevier, 2006.

[35] C. W. Bunn, "The lattice-dimensions of zinc oxide," P PHYS SOC, vol. 47, pp. 835-842, 1935.

[36] E.Mollwo Z. Angew Phys, vol. 6, 1954.

[37] A. R. Hutson, "Piezoelectricity and conductivity in $\mathrm{ZnO}$ and CdS," PHYS REV LETT, vol. 4, pp. 505-507, 1960. 
[38] F.S.Hickernell, "Zinc-oxide thin-film surface-wave transducers," $P$ IEEE, vol. 64, pp. 631-635, 1976.

[39] K. Ueda, H. Tabata, and T. Kawai, "Magnetic and electric properties of transition-metal-doped $\mathrm{ZnO}$ films," APPL PHYS LETT, vol. 79, pp. 988-990, 2001.

[40] S. Pearton, D. Norton, K. Ip, Y. Heo, and T. Steiner, "Recent progress in processing and properties of ZnO," PROG MATER SCI, vol. 50, pp. 293-340, 2005.

[41] Q. Xu, Z. Wen, L. Xu, J. Gao, D. Wu, Shen, T. Qiu, S. Tang, and M. Xu, "Room temperature ferromagnetic pure $\mathrm{ZnO}$," PHYSICA B, vol. 406, pp. 19-23, 2011.

[42] K.-K. Kim, H.-S. Kim, D.-K. Hwang, J.-H. Lim, and S.-J. Park, “Realization of p-type $\mathrm{ZnO}$ thin films via phosphorus doping and thermal activation of the dopant," APPL PHYS LETT, vol. 83, pp. 63-65, 2003.

[43] A. Tsukazaki, A. Ohotomo, T. Onuma, M. Ohtani, T. Makino, M. Sumiya, K. Ohtani, S. F. Chichibu, S. Fuke, Y. Segawa, H. Ohno, H. Koinuma, and M. Kawasaki, "Repeated temperature modulation epitaxy for p-type doping and light-emitting diode based on $\mathrm{ZnO}$," NAT MATER, vol. 4, pp. 42-46, 2005.

[44] L.Schmidt-Mende and J.L.MacManus-Driscoll, "ZnOnanostructures, defects, and devices," MATER TODAY, vol. 10, pp. 40-48, 2007.

[45] R. Hoffman, B. Norris, and J. Wager, "ZnO-based transparent thinfilm transistors," APPL PHYS LETT, vol. 82, pp. 733-735, 2003.

[46] P. Carcia, S. McLean, M. Reilly, and G. Nunes, "Transparent ZnO thin-film transistor fabricated by RF magnetron sputtering," APPL PHYS LETT, vol. 82, pp. 1117-1119, 2003. 
[47] C. Klingshirn, J. Fallert, H. Zhou, J. Sartor, C. Thiele, F. Maier-Flaig, D. Schneider, and $\mathrm{H}$. Kalt, "65 years of $\mathrm{ZnO}$ research-old and very recent results," PHYS STATUS SOLIDI B, vol. 247, pp. 1424-1447, 2010.

[48] C. F. Klingshirm, A. Waag, A. Hoffmann, and J. Geurts, Zinc Oxide: From Fundamental Properties Towards Novel Applications. Springer Series in Materials Science, Vol 120, 2010.

[49] Z. A. Alahmed, First-principles determination of the chemical potentials and vacancy formation energy in lead titanate and barium titanate and structural, electrical, and electromechanical responses in wurtzite $\mathrm{ZnO}$ under in-plane strain. University of Arkansas, 2007.

[50] H.Morkoç and U. Özgür, Zinc Oxide: Fundamentals, Materials and Device Technology. WILEY-VCH Verlag GmbH \& Co. KGaA, Weinheim, 2009.

[51] A. H. Slocum, Precision Machine Design. Society of Manufacturing Engineers, 1992.

[52] A. L. Kholkin, N. A. Pertsev, and A. V. Goltsev, Piezoelectric and Acoustic Materials for Transducer Applications. Springer, 2008.

[53] M.J.Madou, Fundamentals of Microfabrication. CRC-Press, 1997.

[54] S.Zhang, ed., Advances in Materials Science and Engineering: Biological and Biomedical Coatings Handbook Applications. CRC Press, 2011.

[55] V.Srikant and D. Clarke, "On the optical band gap of zinc oxide," J APPL PHYS, vol. 83, pp. 5447-5451, 1998.

[56] D. C. Reynolds, D. C. Look, B. Jogai, C. W. Litton, G. Cantwell, and W. C. Harsch, "Valence-band ordering in ZnO," PHYS REV B, vol. 60, pp. 2340-2344, 1999. 
[57] M. Kadota, T. Miura, and M. Minakata, "Piezoelectric and optical properties of $\mathrm{ZnO}$ films deposited by an electron-cyclotronresonance sputtering system," J CRYST GROWTH, pp. 523-527, 2002.

[58] H.Ibach, "Thermal expansion of silicon and zinc oxide (i)," PHYS STATUS SOLIDI, vol. 31, pp. 625-634, 1969.

[59] J. Lee, H. Kim, C. Hwang, S. Hong, Y. Shin, and N. Lee, “Deposition of $\mathrm{ZnO}$ thin films by magnetron sputtering for a film bulk acoustic resonator," THIN SOLID FILMS, vol. 435, pp. 179-185, 2003.

[60] W.-S. Wang, T.-T. Wu, T.-H. Chou, and Y.-Y. Chen, "A ZnO nanorodbased SAW oscillator," NANOTECHNOLOGY, vol. 20, pp. 1-5, 2009.

[61] T. Gryba, J. Carlier, S. Wang, X. Zhao, S. Guo, and J.-E. Lefebvre, "One port contour-mode $\mathrm{ZnO}$ piezoelectric MEMS resonator," MICROELECTRON ENG, vol. 88, pp. 3003-3010, 2011.

[62] M. K.Wasa and I.Adachi, Thin Films Materials Technology: Sputtering of Compound Materials. William Andrew, Inc, 2004.

[63] M.Ohring, The Materials Science of Thin Films. Academic Press, 1992.

[64] F. Van De Pol, F. Blom, and J. Popma, "RF planar magnetron sputtered ZnO films I: Structural properties," THIN SOLID FILMS, vol. 204, pp. 349-364, 1991.

[65] R.F.Bunshah, Handbook of Deposition Technologies for Films and Coatings: Science, Technology, and Applications. Noyes Publications, 1994.

[66] B. A. Movchan and A. V. Demchishin, "Study of the structure and properties of thick vacuum condensates of nickel, titanium, aluminium oxide, and zirconium dioxide," PHYS MET METALLOGR+, vol. 28:83, 1969. 
[67] J.A.Thornton, "Influence of apparatus geometry and deposition conditions on structure and topography of thick sputtered coatings," $J$ VAC SCI TECHNOL, vol. 11, pp. 666-670, 1974.

[68] L.B.Freund and S.Suresh, Thin Film Materials: Stress, Defect Formation, and Surface Evolution. Cambridge University Press, 2003.

[69] M.F.Doerner and W.D.Nix, "Stresses and deformation processes in thin films on substrates," CRC CR REV SOL STATE, vol. 14, pp. 225$268,1988$.

[70] R.Koch, "The intrinsic stress of polycrystalline and epitaxial thin metal films," J PHYS-CONDENS MAT, vol. 6, p. 9519, 1994.

[71] A. Cimpoiaşu, N. M. van der Pers, T. H. deKeyser, A. Venema, and M. J. Vellekoop, "Stress control of piezoelectric ZnO films on silicon substrates," SMART MATER STRUCT, vol. 5, pp. 744-750, 1996.

[72] S.A.Campbell, The science and engineering of microelectronic fabrication. Oxford University press, 2001.

[73] F.E.Ghodsi and H.Absalan, "Comparative study of $\mathrm{ZnO}$ thin films prepared by different sol-gel route," vol. 118, pp. 659-664, 2010.

[74] K. Ellmer, "Magnetron sputtering of transparent conductive zinc oxide: relation between the sputtering parameters and the electronic properties.," J APPL PHYS D, vol. 33, pp. R17-R32, 2000.

[75] S. Rossnagel, Handbook of Thin Film Deposition Processes and Techniques. William Andrew, Inc, 2001.

[76] B. E. Aufderheide, Coatings Technology Handbook, Third Edition. W. H. Brady Company, 2005.

[77] R.L.Johnson, "Characterization of piezoelectric $\mathrm{ZnO}$ thin films and the fabrication of piezoelectric micro-cantilevers," Master's thesis, Iowa State University, 2005. 
[78] K. Tominaga, N. Ueshiba, Y. Shintani, and O. Tada, "High-energy neutral atoms in the sputtering of ZnO," JPN J APPL PHYS, vol. 20, pp. 519-526, 1980.

[79] N. Fujimura, T. Nishihara, S. Goto, J. Xu, and T. Ito, "Control of preferred orientation for $\mathrm{ZnO} \times$ films: Control of self-texture," J CRYST GROWTH, vol. 130, pp. 269-279, 1992.

[80] Y. Lee, J. Lee, Y. Kim, H. Yang, J. Park, and H. Lim, "Microstructural evolution and preferred orientation change of radio-frequencymagnetron sputtered ZnO thin films," AVS, vol. 14, pp. 1943-1948, 1996.

[81] K.B.Sundaram and A.Khan, "Characterization and optimization of zinc oxide films by RF magnetron sputtering," THIN SOLID FILMS, vol. 295, pp. 87-91, 1997.

[82] W.Water and S.Y.Chu, "Physical and structural properties of $\mathrm{ZnO}$ sputtered films," MATER LETT, vol. 55, pp. 67-72, 2002.

[83] Z. Xingwen, L. Yingwei, L. Yongqiang, M. Ji, and X. Yiben, “Effect of LP-buffer on the structure of $\mathrm{ZnO}$ thin films prepared on glass substrate using RF magnetron sputtering," J MATER SCI-MATER EL, vol. 18, pp. 1021-1025, 2007.

[84] S. Singh, R. Srinivasa, and S. Major, "Effect of substrate temperature on the structure and optical properties of $\mathrm{ZnO}$ thin films deposited by reactive RF magnetron sputtering," THIN SOLID FILMS, vol. 515, pp. 8718-8722, 2007.

[85] C. Li and B. Yang, "Local piezoelectricity and polarity distribution of preferred c-axis-oriented film investigate by piezoresponse force microscopy," J ELECTRON MATER, vol. 40, pp. 253-258, 2011. 
[86] T. Nagase, T. Kamohara, K. Nishikubo, S. Fukamachi, K. Shobu, and M. Akiyama, "Correlation between polarity distribution and piezoelectric response of solution-deposited Zn1-xLixO films," J APPL PHYS, vol. 110, 2011.

[87] "HHV Auto500, HHV ltd, http: / /www.hhvltd.com/."

[88] “University wafer, http:/ / www.universitywafer.com/."

[89] “Valley design, http:/ / www.valleydesign.com/," May 2012.

[90] "Dektak 150 surface profiler, veeco, http://www.veeco.com/."

[91] J. Manifacier, J. Gasiot, and J. Fillard, "A simple method for the determination of the optical constants $n, k$ and the thickness of a weakly absorbing thin film," J PHYS E SCI INSTRUM, vol. 9, pp. 1002-1004, 1976.

[92] F. A. Jenkins and H. E. White, Fundamentals of Optics 4th edition. Mcgraw-Hill Book Company, 1976.

[93] F. Chaabouni, M. Abaab, and B. Rezig, "Effect of the substrate temperature on the properties of $\mathrm{ZnO}$ films grown by magnetron sputtering," MAT SCI ENG B-SOLID, vol. 109, pp. 236-240, 2004.

[94] S.Cho, "Effects of growth temperature on the properties of $\mathrm{ZnO}$ thin films grown by radio-frequency magnetron sputtering," TRANS ELECTR ELECTRON MATER, vol. 10, pp. 185-188, 2009.

[95] Calculated from ICSD using POWD-12++, Reference code: 01-070-8072, 2001.

[96] B. D. Cullity and S. R. Stock, Elements of X-Ray Diffraction. Prentice Hall, 2001. 
[97] R. Hong, J. Huang, H. He, Z. Fan, and J. Shaoo, "Influence of different post-treatments on the structure and optical properties of zinc oxide films," APPL SURF SCI, vol. 242, pp. 346-352, 2005.

[98] M. Chen, Z. Pei, X. Wang, C. Sun, and L. Wen, “Dependence of structural, electrical, and optical properties of $\mathrm{ZnO}: \mathrm{Al}$ films on substrate temperature," J MATER RES, vol. 16, pp. 2118-2123, 2001.

[99] H. Ong, A. Zhu, and G. Du, "Dependence of the excitonic transition energies and mosaicity on residual strain in $\mathrm{ZnO}$ thin films," APPL PHYS LETT, vol. 80, pp. 941-943, 2002.

[100] Y. Lu, W. Hwang, W. Lui, and J. Yang, "Effect of RF power on optical and electrical properties of $\mathrm{ZnO}$ thin film by magnetron sputtering," MATER CHEM PHYS, vol. 72, pp. 269-272, 2001.

[101] D. K. Hwang, K. H. Bang, M. C. Jeong, and J. M. Myoung, “Effects of $\mathrm{RF}$ power variation on properties of $\mathrm{ZnO}$ thin films and electrical properties of p-n homojunction," J CRYST GROWTH, 2003.

[102] S. Lin, J. Huang, and D. Luii, "The effects of RF power and substrate temperature on the properties of $\mathrm{ZnO}$ films," SURF COAT TECH, vol. 176, pp. 173-181, 2003.

[103] J. Molarius, J. Kaitila, T. Pensala, and M. Ylilammi, "Piezoelectric ZnO films by RF sputtering," J MATER SCI-MATER EL, vol. 14, pp. 431-435, 2003.

[104] R. Singh, M. Kumar, and S. Chandra, "Growth and characterization of high resistivity c-axis orientated $\mathrm{ZnO}$ films on different substrates by RF magnetron sputtering for mems applications," J MATER SCI, vol. 42, pp. 4675-4683, 2007.

[105] D. B. Holt and B. G. Yacobi, Extended Defects in Semiconductors Electronic Properties, Device Effects and Structures. Cambridge University Press, 2007. 
[106] D. H.Bach, ed., Thin Films on Glass. Springer-Verlag Berlin Heidelberg, 2003.

[107] “Corning, http:/ /www.catalog2.corning.com."

[108] K.Müller, "Stress and microstructure of sputter deposited thin films: Molecular dynamics investigations," J APPL PHYS, vol. 62, pp. 1796-1799, 1987.

[109] W.D.Nix, "Mechanical properties of thin films," METALL MATER TRANS A, vol. 20, pp. 2217-2245, 1989.

[110] J.-H. Jou, M.-Y. Han, and D.-J. Chen, "Substrate dependent internal stress in sputtered zinc oxide thin films," J APPL PHYS, vol. 71, pp. 4333-4336, 1992.

[111] D. M. Mattox, The foundations of Vacuum Coating Technology. Noyes Publications, 2003.

[112] H. Windischmann, "Intrinsic stress in sputtered thin films," J VAC SCI TECHNOL A, vol. 9, pp. 2431-2436, 1991.

[113] R. Cebulla, R. Wendt, and K. Ellmer, "Al-doped zinc oxide films deposited by simultaneous RF and DC excitation of magnetron plasma: Relationships between plasma parameters and structural and electrical properties," J APPL PHYS, vol. 83, pp. 1087-1095, 1998.

[114] V.Gupta and A.Mansingh, "Influence of post deposition annealing on the structural and optical properties of sputtered zinc oxide," $J$ APPL PHYS, vol. 80, pp. 163-1073, 1996.

[115] Z. Fang, Z. Yan, Y. Tan, X. Liu, and Y. Wang, "Influence of postannealing treatment on the structure properties of $\mathrm{ZnO}$ films," APPL SURF SCI, vol. 241, pp. 303-308, 2004. 
[116] I.Özen and M.Gülgün, "Residual stress relaxation and microstructure in ZnO thin films," ADV SCI TECH, vol. 45, pp. 1316-1321, 2006.

[117] C. R. Aita, A. J. Purdes, R. J. Lad, and P. D. Funkenbush, "The effect of $\mathrm{O} 2$ on reactively sputtered zinc oxide," J APPL PHYS, vol. 51, pp. 5533-5536, 1980.

[118] R.A.Levy, ed., Microelectronic Materials and Processes. Kluwer Academic Publishers, 1986.

[119] Y.Kajikawa, "Texture development of non-epitaxial polycrystalline ZnO films," J CRYST GROWTH, vol. 289, pp. 387-394, 2006.

[120] S.Na and C.Park, "First-principles of the surface energy and the atom cohesion of wurtzite $\mathrm{ZnO}$ and $\mathrm{ZnO}$ : Implications for nanostructure formation," J KOREAN PHYS SOC, vol. 56, pp. 498-502, 2010 . 